\title{
Abstracts from the 53rd European Society of Human Genetics (ESHG) Conference: Oral Presentations
}

(c) European Society of Human Genetics 2020. Modified from the conference website and published with permission 2020

Volume 28 I Supplement 1

Virtual Conference

June 6-9, 2020

Sponsorship: Publication of this supplement was sponsored by the European Society of Human Genetics. All content was reviewed and approved by the ESHG Scientific Programme Committee, which held full responsibility for the abstract selections.

Disclosure Information: In order to help readers form their own judgments of potential bias in published abstracts, authors are asked to declare any competing financial interests. Contributions of up to EUR 10 000.- (Ten thousand Euros, or equivalent value in kind) per year per company are considered "Modest". Contributions above EUR 10 000.- per year are considered "Significant".

Presenting authors are indicated with asterisks in the contributor lists.

\section{Plenary Sessions}

\section{PL1 Opening Plenary}

PL1.2

RNA splicing defects in cancer

R. K. Bradley*

Fred Hutchinson Cancer Research Center, Seattle, WA, United States

Alternative RNA splicing, the process whereby a single gene can give rise to many different proteins, has long been known to be dysregulated in many cancers. Recent studies have provided concrete genetic evidence that splicing dysregulation directly promotes cancer initiation and progression. For example, genes encoding RNA splicing factors are recurrently mutated in both hematologic malignancies and solid tumors. However, uncovering the specific mechanisms by which these mutations promote tumorigenesis has proven challenging. Here, I will discuss recent progress in establishing functional links between somatic mutations affecting RNA splicing factors and tumorigenesis, the promise of correct mis-splicing for cancer therapy, and the recent creation of CRISPR/Cas-based technologies for conducting mRNA isoform-level genetic screens.

R. K. Bradley: None.

\section{PL2 What's New? Highlight Session}

\section{PL2.1}

Evaluation of DNA methylation episignatures for diagnosis and phenotype correlations in $\mathbf{4 2}$ Mendelian neurodevelopmental disorders

E. Aref-Eshghi ${ }^{l}$, J. Kerkhof ${ }^{1}$, V. Pedro ${ }^{2}$, Groupe DI France, M. Barat-Houari ${ }^{3}$, N. Ruiz-Pallares ${ }^{4}$, M. Alders ${ }^{5}, M$.

Mannens $^{5}$, P. Henneman ${ }^{5}$, R. C. Hennekam ${ }^{6}$, A. Ciolfi, S.

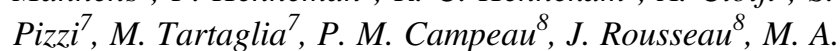
Levy $^{1,2}$, L. Brick ${ }^{9}$, M. Kozenko ${ }^{9}$, T. B. Balci ${ }^{1,2,10}$, V. M.

Siu $^{1,2}$, A. Stuart ${ }^{1}$, M. Kadour ${ }^{1,11}$, J. Masters ${ }^{1,11}$, K. Takano $^{12}$, T. Kleefstra ${ }^{13,14}$, N. de Leeuw ${ }^{13,14}$, M. Field ${ }^{15}$, M. Shaw $^{16}$, J. Gecz ${ }^{16,17}$, G. Merla ${ }^{18}$, P. J. Ainsworth ${ }^{1,2}$, H. Lin $^{1,2}$, D. I. Rodenhiser ${ }^{2,19}$, M. J. Friez ${ }^{20}$, M. Tedder ${ }^{20}$, J. A. 
Lee $^{20}$, B. R. DuPont ${ }^{20}$, R. E. Stevenson ${ }^{20}$, S. A. Skinner ${ }^{20}$, C. E. Schwartz ${ }^{20}$, D. Genevieve G,21 B. Sadikovic $^{1,2^{*}}$

${ }^{1}$ London Health Sciences Centre, London, ON, Canada, ${ }^{2}$ Western University, London, ON, Canada, ${ }^{3}$ Universitaire de Montpellier, Montpellier, France, ${ }^{4}$ Centre Hospitalier Universitaire de Montpellier, Montpellier, France, ${ }^{5}$ Amsterdam University Medical Centers, Amsterdam, Netherlands, ${ }^{6}$ University of Amsterdam, Amsterdam, Netherlands, ${ }^{7}$ Ospedale Pediatrico Bambino Gesù, Rome, Italy, ${ }^{8}$ University of Montreal, Montreal, QC, Canada, ${ }^{9}$ McMaster University, Hamilton, ON, Canada, ${ }^{10}$ Lawson Health Research Institute, London, ON, Canada, ${ }^{11}$ St. Joseph's Health Care, London, ON, Canada, ${ }^{12}$ Shinshu University Hospital, Nagano, Japan, ${ }^{13}$ Radboud University Medical Center, Nijmegen, Netherlands, ${ }^{14}$ Donders Center for Medical Neuroscience, Nijmegen, Netherlands, ${ }^{15}$ Hunter Genetics, Waratah, Australia, ${ }^{16}$ The University of Adelaide, Adelaide, Australia, ${ }^{17}$ South Australian Health and Medical Research Institute, Adelaide, Australia, ${ }^{18}$ IRCCS-Casa Sollievo della Sofferenza, San Giovanni Rotondo, Italy, ${ }^{19}$ Children's Health Research Institute, London, ON, Canada, ${ }^{20}$ Greenwood Genetic Center, Greenwood, SC, United States, ${ }^{21}$ Montpellier University, Montpellier, France

Introduction: Genetic syndromes frequently present with overlapping clinical features and negative or ambiguous genetic findings which can confound accurate diagnosis and clinical management. An expanding number of genetic syndromes have been shown to have unique genomic DNA methylation patterns (episignatures).

Materials and Methods: Peripheral blood episignatures can be used for diagnostic testing as well as for the interpretation of ambiguous genetic test results. We present here an approach to episignature mapping of the largest number of genetic syndromes studied to date $(n=42)$, which has allowed the identification of 34 robust disease-specific episignatures.

Results: We examine emerging patterns of overlap, as well as similarities and hierarchical relationships across these episignatures to highlight their key features as related to genetic heterogeneity, dosage effect, unaffected carrier status, and incomplete penetrance. We demonstrate the necessity of multiclass modeling for accurate genetic variant classification. We demonstrate the utility of this tool in resolving ambiguous clinical cases and identification of previously undiagnosed cases through mass screening of a large cohort of subjects with developmental delays and congenital anomalies. Finally, we outline and present early data from a Canadian national clinical trial EpiSign-CAN currently enrolling 4000 patients to assess impact of this novel diagnostic technology on diagnosis of neurodevelopmental conditions.
Conclusions: This study more than doubles the number of published syndromes with DNA methylation episignatures, and most significantly, opens new avenues for accurate diagnosis and clinical assessment in individuals affected by these disorders.

E. Aref-Eshghi: None. J. Kerkhof: None. V. Pedro: None. M. Barat-Houari: None. N. Ruiz-Pallares: None. M. Alders: None. M. Mannens: None. P. Henneman: None. R. C. Hennekam: None. A. Ciolfi: None. S. Pizzi: None. M. Tartaglia: None. P. M. Campeau: None. J. Rousseau: None. M. A. Levy: None. L. Brick: None. M. Kozenko: None. T. B. Balci: None. V. M. Siu: None. A. Stuart: None. M. Kadour: None. J. Masters: None. K. Takano: None. T. Kleefstra: None. N. de Leeuw: None. M. Field: None. M. Shaw: None. J. Gecz: None. G. Merla: None. P. J. Ainsworth: None. H. Lin: None. D. I. Rodenhiser: None. M. J. Friez: None. M. Tedder: None. J. A. Lee: None. B. R. DuPont: None. R. E. Stevenson: None. S. A. Skinner: None. C. E. Schwartz: None. D. Genevieve: None. B. Sadikovic: None.

\section{PL2.2}

Identity-by-descent detection across 487,409 British samples reveals fine-scale population structure, evolutionary history, and trait associations

J. Nait Saada ${ }^{1 *}$, G. Kalantzis ${ }^{1}$, D. Shyr ${ }^{2}$, M. Robinson ${ }^{3}$, A. Gusev ${ }^{4,5}$, P. Palamara ${ }^{1,6}$

${ }^{1}$ Department of Statistics, University of Oxford, Oxford, United Kingdom, ${ }^{2}$ Department of Biostatistics, Harvard T. H Chan School of Public Health, Boston, MA, United States, ${ }^{3}$ Department of Computer Science, University of Oxford, Oxford, United Kingdom, 'Brigham \& Women's Hospital, Division of Genetics, Boston, MA, United States, ${ }^{5}$ Department of Medical Oncology, Dana-Farber Cancer Institute, Boston, MA, United States, ${ }^{6}$ Wellcome Centre for Human Genetics, University of Oxford, Oxford, United Kingdom

The sharing of identical-by-descent (IBD) genomic segments is a key indicator of genetic relatedness between individuals and plays an important role in a wide range of genomic analyses. We developed a new method, called FastSMC, that enables accurate biobank-scale detection of IBD segments transmitted by common ancestors living up to several hundreds of generations in the past. FastSMC combines a fast heuristic search for IBD segments with accurate coalescent-based likelihood calculations and estimates the age of common ancestors transmitting IBD regions. We applied FastSMC to 487,409 phased samples from the UK Biobank and detected the presence of 214 billion IBD segments transmitted by shared ancestors within 
the past 1,500 years. We quantified time-dependent shared ancestry within and across 120 postcodes, obtaining a finegrained picture of genetic relatedness within the past two millennia in the UK. Sharing of common ancestors strongly correlates with geographic distance, enabling us to localise a sample's birth coordinates from genomic data with a median error of $44 \mathrm{~km}$. We analysed genomic regions with unusually high density of shared ancestry, which is indicative of recent positive selection, and detected 12 genomewide significant signals, including 7 novel sites. We found IBD sharing to be highly predictive of the sharing of ultrarare variants in exome sequencing samples from the UK Biobank, enabling us to detect 29 associations (20 of which novel) with 7 blood-related traits. These results underscore the importance of modelling distant relatedness to reveal subtle population structure, recent evolutionary history, and rare pathogenic variation.

J. Nait Saada: None. G. Kalantzis: None. D. Shyr: None. M. Robinson: None. A. Gusev: None. P. Palamara: None.

\section{PL2.3}

\section{Large-scale targeted sequencing identifies risk genes for} neurodevelopmental disorders

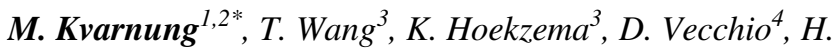
$W_{u^{5}}$, A. Sulovari ${ }^{3}$, B. P. Coe $e^{3}$, M. A. Gillentine,

A. B. Wilfert ${ }^{3}$, A. Lindstrand ${ }^{1,2}$, A. Nordgren $^{1,2}$,

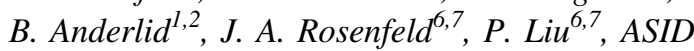

Consortium $^{3}$, I. E. Scheffer ${ }^{8,9,10}$, N. Brunetti-Perri ${ }^{11,12}$,

N. Rommelse ${ }^{13,14}$, D. G. Amaral ${ }^{15}$, G. W. E. Santen ${ }^{16}$,

E. Trabetti ${ }^{17}$, Z. Sedláček ${ }^{18}$, J. J. Michaelson ${ }^{19}$, E. Courchesne $^{20}$, F. R. Kooy ${ }^{21}$, The SPARK Consortium ${ }^{22}$,

C. Romano ${ }^{23}$, H. Peeters ${ }^{24}$, R. A. Bernier ${ }^{25}$, J. Gecz ${ }^{26,27,28}$, K. Xia ${ }^{5,29}$, E. E. Eichler ${ }^{3,30,31}$, M. Nordenskjöld ${ }^{1,2}$

${ }^{1}$ Dept of Molecular Medicine and Surgery, Karolinska Institutet, Stockholm, Sweden, ${ }^{2}$ Department of Clinical Genetics, Karolinska University Hospital, Stockholm, Sweden, ${ }^{3}$ Department of Genome Sciences, University of Washington, Seattle, WA, United States, ${ }^{4}$ Rare Disease and Medical Genetics, AcademDepartment of Pediatrics, Bambino Gesu Children's Hospital, Rome, Italy, ${ }^{5}$ Center of Medical Genetics \& Hunan Key Laboratory of Medical Genetics, School of Life Sciences, Central South University, Changsha, Hunan, China, ${ }^{6}$ Department of Molecular \& Human Genetics, Baylor College of Medicine, Houston, TX, United States, ${ }^{7}$ Baylor Genetics, Houston, TX, United States, ${ }^{8}$ Murdoch Children's Research Institute, Melbourne, Australia, ${ }^{9}$ Department of Paediatrics, Royal Children's Hospital and Department of Medicine, Austin Health, University of Melbourne, Melbourne, Australia, ${ }^{10}$ The Florey Institute of Neuroscience and Mental Health, Parkville, Victoria, Australia, ${ }^{11}$ Department of
Translational Medicine, Federico II University, Naples, Italy, ${ }^{12}$ Telethon Institute of Genetics and Medicine, Pozzuoli, Naples, Italy, ${ }^{13}$ Department of Psychiatry, Donders Institute for Brain, Cognition and Behaviour, Radboud University Medical Center, Nijmegen, Netherlands, ${ }^{14}$ Karakter Child and Adolescent Psychiatry Center, Nijmegen, Netherlands, ${ }^{15}$ Department of Psychiatry and Behavioral Sciences and the MIND Institute, University of California, Davis, Sacramento, CA, United States, ${ }^{16}$ Department of Clinical Genetics, Leiden University Medical Center (LUMC), Leiden, Netherlands, ${ }^{17}$ Department of Neurosciences, Biomedicine and Movement Sciences, University of Verona, Verona, Italy, ${ }^{18}$ Department of Biology and Medical Genetics, Charles University 2nd Faculty of Medicine and University Hospital Motol, Prague, Czech Republic, ${ }^{19}$ Department of Psychiatry, University of Iowa Carver College of Medicine, Iowa city, IA, United States, ${ }^{20}$ Department of Neurosciences, UC San Diego Autism Center, School of Medicine, University of California San Diego, La Jolla, CA, United States, ${ }^{21}$ Department of Medical Genetics, University of Antwerp, Antwerp, Belgium, ${ }^{22}$ Simons Foundation, New York, NY, United States, ${ }^{23}$ Oasi Research Institute-IRCCS, Troina, Italy, ${ }^{24}$ Centre for Human Genetics, KU Leuven and Leuven Autism Research (LAuRes), Leuven, Belgium, ${ }^{25}$ Department of Psychiatry and Behavioral Sciences, University of Washington, Seattle, WA, United States, ${ }^{26}$ Adelaide Medical School and the Robinson Research Institute, the University of Adelaide, South Australia, Australia, ${ }^{27}$ Genetics and Molecular Pathology, SA Pathology, Adelaide, South Australia, Australia, ${ }^{28}$ South Australian Health and Medical Research Institute, Adelaide, South Australia, Australia, ${ }^{29}$ CAS Center for Excellence in Brain Science and Intelligences Technology (CEBSIT), Chinese Academy of Sciences, Shanghai, China, ${ }^{30}$ Howard Hughes Medical Institute, University of Washington, Seattle, WA, United States, ${ }^{31}$ Corresponding author, Seattle, WA, United States

Introduction: Large-scale exome sequencing studies have identified hundreds of genes with significant excess of de novo mutations (DNMs) in neurodevelopmental disorders (NDDs). In this collaborative study, we assessed 125 candidate and high-confidence genes in a large cohort of NDD cases.

Materials and Methods: We targeted 63 candidate genes for resequencing among 16,321 NDD cases and an additional 62 high-confidence genes in 6,211 NDD cases. In order to test for significance at the level of case-control mutation burden and integrating with previously published data, we assessed these genes among NDD cases and compared the mutation burden to nonpsychiatric controls from ExAC. These genes were assessed also in a combined set of 17,426 NDD parent-child trios. Furthermore, 
thorough phenotype studies for seven of the genes with evidence of mutational burden were performed in a subset of the patients.

Results: We identified 48 genes (25 newly reported) with a significant burden of ultra-rare (MAF $<0.01 \%$ ) severe mutations. Among the 125 targeted genes, we show that 90 genes are also enriched for DNMs in parent-child trios. We here focus on seven genes (CTCF, HNRNPU, KCNQ3, ZBTB18, TCF12, SPEN and LEO1) for detailed clinical descriptions to delineate the associated phenotypes and clinical presentation of these specific and newly described neurodevelopmental disorders.

M. Kvarnung: None. T. Wang: None. K. Hoekzema: None. D. Vecchio: None. H. Wu: None. A. Sulovari: None. B. P. Coe: None. M. A. Gillentine: None. A. B. Wilfert: None. A. Lindstrand: None. A. Nordgren: None. B. Anderlid: None. J. A. Rosenfeld: None. P. Liu: None. -. ASID Consortium: None. I. E. Scheffer: None. N. Brunetti-Perri: None. N. Rommelse: None. D. G. Amaral: None. G. W. E. Santen: None. E. Trabetti: None. Z. Sedláček: None. J. J. Michaelson: None. E. Courchesne: None. F. R. Kooy: None. -. The SPARK Consortium: None. C. Romano: None. H. Peeters: None. R. A. Bernier: None. J. Gecz: None. K. Xia: None. E. E. Eichler: None. M. Nordenskjöld: None.

\section{PL2.4}

Genomic and transcriptomic profiling of malformations of cortical development: from tissue to single-cell resolution

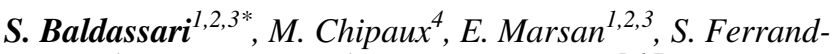 \\ Sorbets $^{4}$, G. Dorfmüller ${ }^{4}, H$. Adle-Biassette ${ }^{5,6,7}, S$. \\ Baulac $^{1,2,3}$
}

${ }^{1}$ ICM Institut du Cerveau et de la Moelle épinière, Paris, France, ${ }^{2}$ Sorbonne Université, UPMC Univ Paris 06, UMR $S$ 1127, INSERM, U1127, Paris, France, ${ }^{3}$ CNRS, UMR 7225, Paris, France, ${ }^{4}$ Department of Pediatric Neurosurgery, Rothschild Foundation Hospital, Paris, France, ${ }^{5}$ INSERM UMR 1141, Hôpital Robert-Debré, Paris, France, ${ }^{6}$ Faculté de Médecine Denis Diderot, Université Paris 7, Paris, France, ${ }^{7}$ Service d'Anatomie et de Cytologie Pathologiques, Hôpital Lariboisière, APHP, Paris, France

Introduction: Malformations of cortical brain development, such as focal cortical dysplasia (FCD) and hemimegalencephaly (HME), are important causes of severe pediatric drug-resistant epilepsies subjected to neurosurgery. At neuropathological assessment, FCD-II subtype is characterized by enlarged dysmorphic neurons (DNs) and balloon cells (BCs) due to mTOR pathway hyperactivation.

Methods and Results: We performed ultra-deep targeted gene sequencing on matched blood-brain resected samples to search for low-allele frequency variants in a panel of mTOR pathway and FCD genes in 99 FCD patients. We report that $\sim 30 \%$ of FCD-I patients carried somatic loss-offunction SLC35A2-variants and $\sim 60 \%$ of FCD-II/HME patients presented somatic gain-of-function MTOR, AKT3, $P I K 3 C A$ and $R H E B$-variants or two-hit, germline and somatic loss-of function DEPDC5, NPRL3, TSC1 and TSC2 variants. Analysis by droplet digital PCR of microdissected cells demonstrated that only DNs and BCs in FCD-II/HME carry the pathogenic variants, and that lossof-heterozygosity in DNs in DEPDC5-mutated cases represents a mutational mechanism in FCD. Global unsupervised analysis of RNAseq from frozen bulk tissues from 20 mutated patients showed clusters independent of the nature of the variant/gene. We therefore performed single-nuclei RNAseq from mutated frozen resected brain tissues to better resolve the mosaic nature of the lesions and investigate the cellular heterogeneity characterizing FCD. Cell-type specific transcriptomic profiling revealed novel insights into epilepsy pathogenic mechanisms.

Conclusions: Our novel findings represent an important advancement in understanding the genetic etiology and mechanisms underlying epilepsies associated to malformations of the cortical development. This work was funded by the ERC grant n.682345 and Easi-Genomics Access grant

S. Baldassari: None. M. Chipaux: None. E. Marsan: None. S. Ferrand-Sorbets: None. G. Dorfmüller: None. H. Adle-Biassette: None. S. Baulac: None.

\section{PL2.5}

TAD-shuffling at the FGF8 locus causes Split-Hand/ Foot Malformation type 3

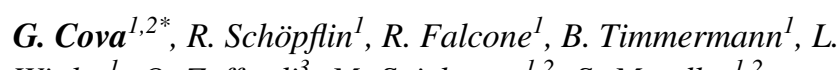

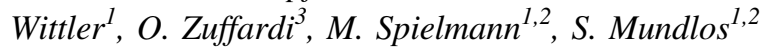

${ }^{1}$ Max Planck Institute for Molecular Genetics, Berlin, Germany, ${ }^{2}$ Institute for Medical Genetics and Human Genetics, Charité - Universitätsmedizin Berlin, Berlin, Germany, ${ }^{3}$ Department of Molecular Medicine, University of Pavia, Pavia, Italy

In recent years chromosome conformation capture assays such as $\mathrm{Hi}-\mathrm{C}$ revealed that mammalian genomes are organized in genomic units called topologically associated domains (TADs). TADs provide the regulatory scaffold for physical contacts between regulatory elements and their target genes. Structural variations, such as duplications, deletions and inversions, can result in disruptions of TAD structures, gene misexpression and consequently in human diseases. Split-Hand/Foot Malformation type 3 is a rare congenital limb defect affecting the central digits of hands and feet and has been associated with tandem duplications 
at the FGF8 locus. In order to investigate the molecular pathomechanism underlying this disease we mapped 25 duplications and one inversion at the $F G F 8$ locus and we generated mice carrying the corresponding rearrangements using the CRISPR/Cas9 system. Capture Hi-C maps spanning the Fgf8 locus revealed the presence of ectopic interactions between the $F g f 8$ endogenous regulatory elements and an unrelated gene within the locus in mutant mice compared to wild-type. We showed that both in the tandem duplications and the inversion, the $F g f 8$ enhancers are relocated into a new TAD causing misexpression of the neighbouring gene in an $F g f 8$-like pattern in the limb bud. This ectopic expression in the apical ectodermal ridge of developing limbs is associated with the limb phenotype observed in mice and in human patients. Therefore, our functional data show that TAD-shuffling at the $F g f 8$ locus is the cause of this human limb malformation syndrome and further highlight this new mutational mechanism and its role in human disease.

G. Cova: None. R. Schöpflin: None. R. Falcone: None. B. Timmermann: None. L. Wittler: None. O. Zuffardi: None. M. Spielmann: None. S. Mundlos: None.

\section{PL2.6}

The enrichment of breakpoints in late-replicating chromatin provides novel insights into chromoanagenesis mechanisms
N. Chatron ${ }^{1,2,3^{*}}$, G. Giannuzzi $i^{3}$, P. Rollat-Farnier ${ }^{1,4}$, F. Diguet ${ }^{1,2}$, E. Porcu ${ }^{3}$, T. Yammine ${ }^{1,2}$, K. UGUEN ${ }^{5}$, Z. Bellil ${ }^{1,2}$, J. Lauer Zillhardt ${ }^{6}$, A. Sorlin ${ }^{7}$, F. Ader ${ }^{8}$, A. Afenjar ${ }^{9}$, J. Andrieux ${ }^{10}$, C. Bardel ${ }^{4}$, E. Calpena $^{11}$, S. Chantot-Bastaraud ${ }^{12}, P$. Callier ${ }^{7}$, N. Chelloug ${ }^{13}$, E. Chopin ${ }^{14}$, M. Cordierl', C. Dubourg ${ }^{15,16}$, L. Faivre $^{17}$, F. Girard ${ }^{18}$, S. Heide ${ }^{19}$, Y. Herenger ${ }^{20}$, S. Jaillard ${ }^{21}$, B. Keren ${ }^{19}$, S. J. L. Knight ${ }^{22}$, J. Lespinasse ${ }^{23}$, L. Lohmann ${ }^{24}$, N. Marle ${ }^{7}$, R. Maroofian ${ }^{25}$, A. Masurel-Paulet ${ }^{17}$, M. Mathieu-Dramard ${ }^{26}$, C. Metay ${ }^{27}$, A. T. Pagnamenta ${ }^{22}$, M. Portnoi ${ }^{12}$, F. Prieur ${ }^{28}$, M. Rio ${ }^{29}$, J. Siffroi ${ }^{30}$, S. Valence ${ }^{31,32}$, J. C. Taylor ${ }^{22}$, A. O. M. Wilkie ${ }^{11}$, P. Edery ${ }^{1,2}$, A. Reymond ${ }^{3}$, D. Sanlaville ${ }^{1,2}$, C. Schluth- Bolard $^{l, 2}$

${ }^{1}$ Service de Génétique, Hospices Civils de Lyon, Lyon, France, ${ }^{2}$ Equipe GENDEV, CRNL, INSERM U1028, CNRS UMR5292 UCBL1, Lyon, France, ${ }^{3}$ Center for Integrative Genomics, University of Lausanne, Lausanne, Switzerland, ${ }^{4}$ Cellule Bioinformatique Plateforme NGS, Hospices Civils de Lyon, Lyon, France, ${ }^{5}$ Service de Génétique, CHU Brest, Brest, France, ${ }^{6}$ Unité de Diagnostic Préimplantatoire, Laboratoires de Diagnostic Génétique, Hôpitaux Universitaires de Strasbourg, Strasbourg, France, ${ }^{7}$ Laboratoire de génétique chromosomique et moléculaire, CHU de Dijon,
Dijon, France, ${ }^{8}$ AP-HP, Département de Génétique médicale, Cardiogénétique et myogénétique moléculaire et cellulaire, Hôpital de la Pitié-Salpêtrière, Paris, France, ${ }^{9} A P-H P$, Département de Génétique médicale, UF de Génétique clinique, hôpital d'Enfants Armand Trousseau, Paris, France, ${ }^{10} \mathrm{CHRU}$ de Lille, Service de génétique, Hôpital Jeanne de Flandre, Lille, France, ${ }^{11}$ Clinical Genetics Group, MRC Weatherall Institute of Molecular Medicine, University of Oxford, Oxford, United Kingdom, ${ }^{12} A P-H P$, Département de Génétique médicale, UF de Génétique chromosomique, hôpital d'Enfants Armand Trousseau, Paris, France, ${ }^{13}$ Laboratoire de cytogénétique, Service de génétique médicale, CHRU Tours, Tours, France, ${ }^{14}$ Centre de Biotechnologie Cellulaire, Hospices Civils de Lyon, Lyon, France, ${ }^{15}$ Service de Génétique Moléculaire et Génomique médicale, CHU Rennes, Rennes, France, ${ }^{16}$ IGDR, CNRS UMR 6290, Université de Rennes 1, Rennes, France, ${ }^{17}$ Centre de génétique, Hôpital d'enfants, CHU de Dijon, Dijon, France, ${ }^{18}$ Laboratoire de cytogénétique constitutionnelle et prénatale, CHU Strasbourg, Strasbourg, France, ${ }^{19}$ APHP, Département de Génétique et Centre de Référence Déficiences Intellectuelles de Causes Rares, Hôpital de la Pitié-Salpêtrière, Paris, France, ${ }^{20}$ Service de génétique médicale, CHRU Tours, Tours, France, ${ }^{21}$ Service de cytogénétique et biologie cellulaire, CHU Rennes, Rennes, France, ${ }^{22}$ Oxford NIHR Biomedical Research Centre, Wellcome Centre for Human Genetics, University of Oxford, Oxford, United Kingdom, ${ }^{23}$ Service de génétique, CH Métropole Savoie, Chambéry, France, ${ }^{24}$ Département de génétique, Laboratoire CERBA, Saint-Ouen l'Aumône, France, ${ }^{25}$ Institute of Neurology, University College London, London, United Kingdom, ${ }^{26} \mathrm{Ser}$ vice de génétique clinique et oncogénétique, CHU AmiensPicardie, Amiens, France, ${ }^{27}$ AP-HP, Hôpital Henri Mondor, Créteil, France, ${ }^{28}$ Service de Génétique Clinique, Chromosomique et Moléculaire, CHU Hôpital Nord, Saint-Etienne, France, ${ }^{29} \mathrm{AP}-\mathrm{HP}$, Service de Génétique Médicale, Hôpital Necker-Enfants Malades, Paris, France, ${ }^{30} A P-H P$, Département de Génétique médicale, UF de Génétique chromosomique, hôpital d'Enfants Armand Trousseau, Paris, Paris, France, ${ }^{31}$ Sorbonne Université, GRC $n^{\circ} 19$, pathologies Congénitales du Cervelet-LeucoDystrophies, Paris, France, ${ }^{32}$ AP-HP, Hôpital Armand Trousseau; GHUEP, Service de Neurologie Pédiatrique, Paris, France

The rise of pangenomic molecular assays allowed uncovering complex rearrangements named chromoanagenesis that were hypothesized to result from catastrophic shattering events. Constitutional cases have typically been reported individually preventing identification of common features and uncovering the mechanisms at play. We characterized 20 new chromoanagenesis and discovered yet-undescribed features. While literature differentiates chromothripsis and its shattering event repaired through non-homologous end joining from 
chromoanasynthesis born to aberrant replicative processes, we identified shattered chromosomes repaired through a combination of mechanisms. In particular, three samples present with "rearrangement hubs" comprising a fragmented kilobase-long sequence threaded throughout the rearrangement. To assess the mechanisms at play, we merged our data with those of 20 published constitutional complex chromosomal rearrangement cases. We evaluated if the distribution of their 1033 combined breakpoints was distinctive using bootstrap simulations and found that breakpoints tend to keep away from haplosensitive genes suggesting selective pressure. We then compared their distribution with that of 13,310 and 468 breakpoints of cancer complex chromosomal rearrangements and constitutional simple rearrangement samples, respectively. Both complex rearrangement groups showed breakpoint enrichment in late replicating regions suggesting similar origins for constitutional and cancer cases. Simple rearrangement breakpoints but not complex ones were depleted from lamina-associated domains (LADs), possibly as a consequence of reduced mobility of DNA ends bound to lamina. The enrichment of breakpoints in late-replicating chromatin for both constitutional and cancer chromoanagenesis provides an orthogonal support to the premature chromosome condensation hypothesis that was put forward to explain chromoanagenesis.

N. Chatron: None. G. Giannuzzi: None. P. Rollat-Farnier: None. F. Diguet: None. E. Porcu: None. T. Yammine: None. K. Uguen: None. Z. Bellil: None. J. Lauer Zillhardt: None. A. Sorlin: None. F. Ader: None. A. Afenjar: None. J. Andrieux: None. C. Bardel: None. E. Calpena: None. S. ChantotBastaraud: None. P. Callier: None. N. Chelloug: None. E. Chopin: None. M. Cordier: None. C. Dubourg: None. L. Faivre: None. F. Girard: None. S. Heide: None. Y. Herenger: None. S. Jaillard: None. B. Keren: None. S. J. L. Knight: None. J. Lespinasse: None. L. Lohmann: None. N. Marle: None. R. Maroofian: None. A. Masurel-Paulet: None. M. Mathieu-Dramard: None. C. Metay: None. A. T. Pagnamenta: None. M. Portnoï: None. F. Prieur: None. M. Rio: None. J. Siffroi: None. S. Valence: None. J. C. Taylor: None. A. O. M. Wilkie: None. P. Edery: None. A. Reymond: None. D. Sanlaville: None. C. Schluth-Bolard: None.

\section{PL3 ELPAG Award Lecture}

\section{PL3.1}

\section{ELPAG Award Lecture}

\section{H. Skirton*}

\section{University of Plymouth (retired), Plymouth, United Kingdom}

The definition of genetic counselling includes the important terms, 'psychological' and 'adaptation'. While practising clinically, I started my research work because I wondered what made a difference to the family's ability to cope and adapt. In this presentation I will discuss early work that influenced me, my own research journey and thoughts for the future of research in genetic counselling. My own studies, undertaken from 1994 to 2019, have reinforced my belief that, although communicating scientific information is important, addressing the psychological issues that inevitably arise alongside a genetic diagnosis is essential to enable the family to adjust and adapt the information to their own lives. My doctoral study, for example, showed that people had difficulty in applying the scientific information to their personal circumstances unless they believed that the practitioner related to them as an individual, and that improving psychological peace of mind was a major motivator for those using genetic services. Despite the clinical advances of the past four decades, studies still indicate that family members fail to communicate genetic risk to each other, that genetic decision making can still be fraught with distress and ambivalence and a proportion of those who could benefit from screening and/or treatment fail to seek it. I would argue that addressing these issues still falls within the remit of genetic health professionals, particularly genetic counsellors, but there is a dearth of research, particularly using qualitative methods, to inform current practice. Genetic counsellors can be overwhelmed by the ever-expanding body of genomic science underpinning our work. However, there is a continuing challenge for counsellors in this ultra-information era to offer psychological support to family members to enable information to be utilised, support family communication and to reach those who are unaware of their options. I emphasise the need for more applied research, especially addressing questions initiated by those engaged in clinical practice and using qualitative methods where appropriate.

H. Skirton: None.

\section{Concurrent Symposia}

\section{S01 What to Expect from Prenatal Diagnosis}

S01.1

Prenatal Genomic Approach to Genetic Diseases of Bone

\section{Krakow*}

\section{UCLA, Los Angeles, CA, United States}

Skeletal dysplasia are a heterogenous group of mendelian disorders that often present in the prenatal period. With the expansion of available genomic technologies, many of these techniques have been applied to both the preimplantation and prenatal periods. These methodologies include noninvasive 
prenatal testing for de novo or dominantly inherited skeletal disorders, couple screening for recessively inherited skeletal diseases, PGT for familial inherited disorders. microarray analysis for copy number variation, gene panels for skeletal dysplasias and related diseases, and exome analysis. The disease genes have been identified for most of the $>460$ established skeletal dysplasias, thus affording the opportunity to address the molecular basis of most of the skeletal disorders that will present in the prenatal period. Use of exome analysis has identified previously unappreciated prenatal onset disorders, expanding the number of defined skeletal disorders. However, in spite of the advances in genomic technologies, the mainstay of prenatal diagnosis remains prenatal ultrasound evaluation. Most cases of skeletal dysplasia are detected by prenatal ultrasound and appreciation of the ultrasound findings helps guide diagnosis and genomic analyses. Key ultrasound findings including lethality can help guide management pending the results of molecular diagnosis.

D. Krakow: None.

\section{S01.2}

\section{Pandora's Pregnancy - A new Era for Prenatal Genetic Testing}

\section{Y. Hashiloni-Dolev ${ }^{1 *}$, T. Nov-Klaiman ${ }^{2}$, A. Raz ${ }^{3}$}

${ }^{1}$ Ben-Gurion University of the Negav, Israel, Tel Aviv, Israel, ${ }^{2}$ Ben-Gurion University of the Negav, Israel, Munich, Israel, ${ }^{3}$ Ben-Gurion University of the Negav, Israel, Be'er Sheva, Israel

The talk is based on conceptual analysis of seminal works that shaped the socio-ethical discourse surrounding the experiences of parents as well as professionals with prenatal diagnosis in the last thirty years. It delineates a shift from the "traditional" technologies of karyotyping in PND to the current phase of advanced genetic technologies including non-invasive prenatal testing (NIPT), chromosomal microarray analysis (CMA) and whole-exome sequencing (WES) with their higher detection rate and related abundance of uncertain data. The talk will consider the implications of this new era of PND for patients and health professionals by drawing on previous studies documenting how probability and uncertainty affect informed consent/choice, health risks communication, customer satisfaction and decision-making, and parent-child bonding. I will argue that these changes move us beyond the idioms and realities of the "tentative pregnancy" and "moral pioneering", to uncertainty, probability-based counseling and moral/translational gambling. I will conclude by discussing what is needed to maintain hope in the era of Pandora's pregnancy.

Y. Hashiloni-Dolev: None. T. Nov-Klaiman: None. A. Raz: None.
S02 Liquid Biopsy for Early Detection of Cancer

\section{S02.1}

Methylation Patterns of Plasma DNA in Health and Disease

\section{Y. Dor}

\section{The Hebrew University of Jerusalem, Jerusalem, Israel}

Each cell type has a unique and stable DNA methylation pattern, reflecting its gene expression program. We have established a comprehensive atlas of tissue and cell typespecific human methylomes, allowing to define multiple loci that are unmethylated or methylated specifically in a given cell type. We are using these loci as biomarkers to identify the tissue origins of cfDNA, and to infer physiologic and pathologic tissue dynamics. I will discuss examples for the use of tissue specific cfDNA methylation patterns including the detection of cardiomyocyte cfDNA after myocardial infarction and in sepsis; the detection of tissue-specific cfDNA in patients with early and late stage cancer; and a dissection of the phenomenon of elevated cfDNA after intensive exercise.

Y. Dor: None.

\section{S03 Our Ancestry}

\section{S03.2}

What ancient humans can teach us about modern genetic variation

J. Kelso ${ }^{1,2 *}$

${ }^{1}$ Leipzig, Germany, ${ }^{2}$ Max Planck Institute for Evolutionary Anthropology, Leipzig, Germany

Recent technological advances have made it possible to recover genome sequences from a number of archaic and early modern humans. These genomes offer a unique opportunity to identify genetic changes that have come to fixation or reached high frequency in modern humans since the divergence from our common ancestor with Neandertals and Denisovans. Analyses of these archaic genomes have also provided direct evidence for interbreeding between early modern and archaic humans. As a result all presentday people outside of Africa carry approximately $2 \%$ Neandertal DNA, and some populations, largely in Oceania, also carry DNA from Denisovans. This introgressed DNA has been shown to have both positive and negative outcomes for present-day carriers: underlying apparently adaptive phenotypes as well as influencing disease risk. In recent work we have identified Neandertal haplotypes that 
are likely of archaic origin and determined the likely functional consequences of these haplotypes using public genome, gene expression, and phenotype datasets. We have also used simulations, as well as the distribution of Neandertal DNA in ancient modern humans, to understand how selection has acted on Neandertal introgressed sequences over the last 45,000 years.

\section{S04 Public and Patient Engagement}

\section{S04.1}

\section{The Patient's Role in New Disease Gene Discovery}

\section{P. Schaaf*}

\section{University Hospital Heidelberg, Heidelberg, Germany}

The practice of clinical genetics has been revolutionised by the implementation of next generation sequencing. Novel disease genes are recognised on a weekly basis, and the number of OMIM-listed disease-gene associations continues to increase steadily.

What are the characteristics of "patient no.1", and how do you engage with your patient families to work together beyond the initial disease gene discovery?

Here, we highlight the discovery of Schaaf-Yang syndrome, a rare neurodevelopment disorder caused by truncating mutations in MAGEL2, a protein-coding gene within the Prader-Willi syndrome domain on chromosome 15q11.2. We highlight ongoing physician-patient interactions, leading to a deeper understanding of disease phenotype, genotype-phenotype interactions, but also molecular mechanisms and avenues towards therapeutic development.

C. P. Schaaf: None.

\section{S04.2}

Experiences with public engagement: Estonian Biobank's example

A. Allik $^{1,2 *}$, A. Metspalu $^{2}$, L. Leitsalu ${ }^{2}$, L. Milani $^{2}$

${ }^{1}$ Tartu, Estonia, ${ }^{2}$ Estonian Genome Center, Institute of Genomics, University of Tartu, Tartu, Estonia

Introduction: The Estonian government launched a program in 2018 to map the microarray-based genotypes of 100,000 out of 1.3 million inhabitants. The goal of this initiative was to introduce the collected genotype data in Estonia's upgraded healthcare system for delivering personalized medicine information. Estonia's healthcare system has been revolutionized by innovative e-solutions and has become beneficial for patients, doctors, hospitals, and also the government for its convenient accessibility and reasonable maintenance costs. Each citizen in Estonia has an online e-Health record that can be accessed with the person's electronic ID-card which makes the e-Health medical record system secure and the information easily accessible to authorized individuals. The project has been a huge challenge for the Estonian Biobank, considering that the first set of 52,000 biobank participants were recruited over a 10-year period (2002-2011). The new recruitment started in 2018, afterwards an additional $150+$ thousand people have joined the biobank. In total, the biobank participants cover approximately $20 \%$ of the adult population of Estonia. Long before the new recruitment, in 2015, Estonian Biobank started providing personalized genetic feedback to the biobank participants and an interest towards it is huge. by now Around 3000 people have received their feedback reports by now. As a result, the government of Estonia has launched the Personalized medicine program and invested 5, million $€$ for developing state-of-the-art digital decision support system to use the gathered genomic information in the Estonian healthcare system.

Methods: In order to recruit an additional $15 \%$ of the adult population of Estonia, traditional marketing tools were applied to gain public attention and support to benefit the nationwide project with a minimized budget. Our approaches included: explaining the benefits of using the genetic information in healthcare system by public media, involving social media influencers, and arranging social media challenges to draw the attention and raise interest to the project. Enrolment consisted of digitally signed consents using Estonian ID-card systems, and blood sample donations collected through a wide network of pharmacies.

Results: By the end of 2019, there are 200,000+ biobank participants, increasing the biobank cohort from previous $5 \%$ to $20 \%$ of adult population of Estonia in less than two years.

Conclusion: A lot can be done if you think out-of-thebox! The approaches we used and experiences gained throughout this project can be successfully used by other countries.

A. Metspalu: None. L. Leitsalu: None. L. Milani: None.

\section{S04.3}

My DNA, whose business is it anyway? A Citizen Forum on the Societal Impact of Genomics

\section{W. Van Hoof*, C. Mayeur}

Sciensano, Brussels, Belgium

Precision medicine, personalized screening, prevention, ever-expanding commercial services and the need for 
massive data sharing in genomics have led to a need to engage the public in a discussion on the governance of genomic information. Therefore, Sciensano and the King Baudouin Foundation organized a Belgium citizen forum on the ethical, legal, and societal implications (ELSI) surrounding the use of genomic information. This deliberative forum resulted in policy recommendations for the Belgian Minister of Public Health, related experts and stakeholders. The citizen forum consisted of an issue framing workshop, where experts indicated which questions the citizens needed to answer. This was followed by the citizen forum, where 32 citizens discussed these questions over three weekends, while being informed by a neutral information package and a wide range of resource persons. Finally, the recommendations of the citizens formed the basis for a stakeholder workshop, where experts and policy makers discussed the potential implementation of these recommendations into practice. The entire process was supported by an expert committee and audited by two independent researchers. The qualitative analysis of the citizen forum showed that the citizen panel talked about the genome as their property because it is the most intimate part of an individual. Based on this statement, citizens require autonomy in decision-making related to the use of their genomic information and consider that it is society's responsibility to provide citizens with the rights of individual control, transparency, and traceability in the uses of this information. Citizens must use these rights responsibly to protect their privacy. The citizen panel strongly believes that "Individuals are more than their genome and should not be prisoners of it". To this end, protection against any form of discrimination, categorization, and rejection of individuals based on their genetic makeup must be established. The citizens assert that there are many societal responsibilities to protect citizens from downsides and promote upsides from the use of genomic information: the precautionary principle should guide the way in the development of a framework for genomics. Within a well-developed framework that inspires trust around the use of genomic data, solidarity and reciprocity are core values that citizens support in the context of genomic data sharing. Citizens identify a range of individual responsibilities in decision making in genomics and accept that societal nudges might be required to balance personal interests and the common good. This citizen forum shows that citizens can make meaningful contributions to a complex field like ELSI in genomics. Continued citizen involvement of the public in the governance of genomic information in society is vital. It ensures that societal norms and values guide the technology and not the other way around.

W. Van Hoof: None. C. Mayeur: None.
S05 Development and Disease at Single Cell Resolution

\section{S05.1}

Embryo-scale, single-cell spatial transcriptomics

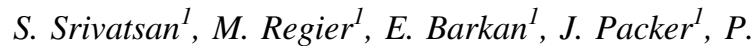

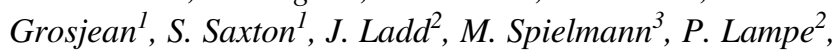
J. Shendure ${ }^{1,4,5}$, K. Stevens ${ }^{1}$, C. Trapnell ${ }^{4,6^{*}}$

${ }^{1}$ University of Washington, Seattle, WA, United States, ${ }^{2}$ Fred Hutch Cancer Research Center, Seattle, WA, United States, ${ }^{3}$ Max Planck Institute for Molecular Genetics, Berlin, Germany, ${ }^{4}$ Brotman Baty Institute for Precision Medicine, Seattle, WA, United States, ${ }^{5}$ Howard Hughes Medical Institute, Seattle, WA, United States, ${ }^{6}$ Seattle, WA, United States

Spatial patterns of gene expression span many scales, and are shaped by both local (e.g. cell-cell interactions) and global (e.g. tissue, organ) context. However, most in situ methods for profiling gene expression either average local contexts or are restricted to limited fields of view. Here we introduce sciSpace, a scale-flexible method for spatial transcriptomics that retains single cell resolution while simultaneously capturing heterogeneity at larger scales. As a proof-of-concept, we apply sci-Space to the developing mouse embryo, capturing the approximate spatial coordinates of profiled cells from whole embryo serial sections. We identify genes including Hox-family transcription factors expressed in an anatomically patterned manner across excitatory neurons and other cell types. We also show that sci-Space can resolve the differential contribution of cell types to signalling molecules exhibiting spatially heterogeneous expression. Finally, we develop and apply a new statistical approach for quantifying the contribution of spatial context to variation in gene expression within cell types.

S. Srivatsan: None. M. Regier: None. E. Barkan: None. J. Packer: None. P. Grosjean: None. S. Saxton: None. J. Ladd: None. M. Spielmann: None. P. Lampe: None. J. Shendure: None. K. Stevens: None.

\section{S05.2}

Cellular drivers of injury response and regeneration in the zebrafish heart

\section{J. P. Junker ${ }^{*}$}

Berlin Institute for Medical Systems Biology (BIMSB), Berlin, Germany

A key goal of developmental biology is to understand how a single cell transforms into a full-grown organism consisting 
of many cells. Although impressive progress has been made in lineage tracing using imaging approaches, analysis of vertebrate lineage trees has mostly been limited to relatively small subsets of cells. Here we present LINNAEUS, a strategy for massively parallel lineage tracing on the single cell level. We exploit the fact that Cas9-induced generation of double-strand breaks leads to formation of short insertions or deletions ("genetic scars") that are variable in their length and position. We demonstrate that these genetic scars have very high complexity, making them ideal cellular barcodes for lineage analysis. Reading out scar sequences by single-cell RNA-seq enables us to simultaneously measure lineage relationships and transcriptome profiles in thousands of single cells. We introduce novel computational methods that address the major challenges of CRISPR/Cas9-based lineage tracing, such as preferential creation of specific scar sequences and stochastic dropout events. LINNAEUS allows scaling up lineage analysis to the level of the whole organism, which makes it an ideal approach for studying processes like organ regeneration after injury. Here, we use LINNAEUS to identify the origin and function of transient cell types that are generated upon heart injury, and we identify novel cellular drivers of the regeneration process.

J. P. Junker: None.

\section{S05.3}

\section{Transcriptome-Scale Super-Resolved Imaging in Tis-} sues by RNA SeqFISH

\section{Cai}

\section{Caltech, Pasadena, CA, United States}

Imaging the transcriptome in situ with high accuracy has been a major challenge in single cell biology, particularly hindered by the limits of optical resolution and the density of transcripts in single cells. We developed seqFISH+, that can image the mRNAs for 10,000 genes in single cells with high accuracy and sub-diffraction-limit resolution, in the mouse brain cortex, subventricular zone, and the olfactory bulb, using a standard confocal microscope. The transcriptome level profiling of seqFISH+ allows unbiased identification of cell classes and their spatial organization in tissues. In addition, seqFISH+ reveals subcellular mRNA localization patterns in cells and ligand-receptor pairs across neighboring cells. This technology demonstrates the ability to generate spatial cell atlases and to perform discovery-driven studies of biological processes in situ.

L. Cai: None.
S06 Making Sense out of Nonsense

S06.1

Nonsense Mediated Decay in Neuronal Development and Disease

\section{Wilkinson*}

\section{Univerity of California, San Diego, CA, United States}

Nonsense-mediated RNA decay (NMD) is a highly selective and conserved RNA turnover pathway that promotes the decay of specific subsets of RNAs. A critical factor in the NMD pathway is UPF3B, an adapter protein that binds with other NMD proteins to form a molecular complex that drives rapid RNA decay. In humans, mutations in UPF3B cause intellectual disability (ID) and are strongly associated with autism, attention-deficit hyperactivity disorder, and schizophrenia (SCZ). To understand UPF3B's role in vivo, we generated a mouse model with a null mutation in the Upf3b gene. These Upf3bnull mice are viable but exhibit a profound defect in pre-pusle inhibition (PPI), a measure of sensomotor gating commonly deficient in individuals with SCZ and other brain disorders. These NMD-deficient mice also have deficits in specific forms of learning and memory. Consistent with both their PPI and learning defects, specific neuronal cell subsets in Upf3b-null mice display deficient dendritic spine maturation in vivo. Neural stem cells from Upf3b-null mice have impaired ability to undergo differentiation and require prolonged culture to give rise to functional neurons with electrical activity. During the course of our analysis, we discovered that Upf3b-null mice also have a phenotype consistent with a partial olfactory defect. This provided an opportunity to examine the role of Upf3b in olfactory neurogenesis, a simple and easily tractable neural developmental system. Single-cell RNA sequencing (scRNAseq) analysis revealed that Upf3b-null mice have specific defects in olfactory neurogenesis, including in olfactory stem cells. These NMD-deficient mice also had deficits in the olfactory receptor (OR) repertoire in olfactory sensory neuronal subsets at different stages of development. The nature of these defects strongly suggested that NMD has a key role in the OR selection process. We suggest that NMD-deficient Upf3b-null mice will be a useful model system to decipher the roles of NMD in neural development and function in vivo.

M. Wilkinson: None.

S06.3

Unproductive splicing and ultraconserved nonsense

\section{Lareau $^{*}$}

University of California, Berkeley, Berkeley, CA, United States 
The most conserved regions of any human genes are found within alternative exons that act as switches to turn off expression of SR splicing factors. When these 'poison' exons are spliced into SR gene transcripts, they trigger mRNA degradation by nonsense-mediated decay (Lareau et al, 2007; Ni et al, 2007). This shifts the mRNA output of the gene away from the productive, protein-coding form, allowing post-transcriptional downregulation of SR protein expression. However, despite the intense conservation, the functional impact of this regulation is unknown. We set out to identify developmental processes showing changes in the splicing of poison exons in SR genes. Using bulk and singlecell RNA-seq data, we found that the inclusion of poison exons changes dramatically during neurogenesis, and that the splicing patterns of different SR poison exons follow distinct temporal profiles. To identify trans-acting regulators of poison exon inclusion, we used publicly available CLIPseq data from 157 RNA-binding proteins to train motifbased classifiers and predict binding in and near the poison exons whose splicing changes during neurogenesis. We found that SR splicing factors form a highly interconnected network by binding both to their own poison exons and to the poison exons of other SR splicing factors. During neurogenesis, the expression of these splicing factors is tightly coordinated with the splicing of several groups of exons to which they bind. We identified specific regions within SR poison exons that are bound by one or several splicing regulators which may act in concert or in opposition. We are removing these regions, as well as full poison exons, to measure their impact on neuronal differentiation. Our results indicate that poison exons function as an auto- and crossregulatory mechanism that tightly regulates the levels of splicing factors during neurogenesis, and that this regulation is mediated by ultraconserved sequences.

L. Lareau: None.

\section{S07 Abstracts Withdrawn}

\section{S08 Cardiovascular Genetics}

\section{S08.1 \\ Challenges in Diagnostic Sequencing for Inherited Cardiac Conditions}

\section{R. Walsh}

Amsterdam UMC, Amsterdam, Netherlands

Diagnostic genetic sequencing has become an integral part of clinical management for most inherited cardiac diseases. Although identifying the Mendelian variants underlying disease will inform treatment in only a minority of cases, it can be used to confirm diagnosis and enable cascade screening in patients' families. However there are a number of challenges associated with cardiovascular diagnostic sequencing that have limited its effectiveness. The era of candidate gene studies led to a plethora of weak and false gene-disease associations, and subsequently a large increase in uncertain (and even false positive) findings when incorporated into diagnostic panels. Recent efforts through community curation initiatives such as ClinGen and large-scale rare variant association studies have clarified many of these associations, enabling more focused and accurate diagnostic testing panels. Variant interpretation remains a critical and often rate-limiting step - although widespread adoption of the ACMG/AMP guidelines have brought standardisation and stringency to variant classification, they require customisation for specific diseases and genes and can lead to the under-calling of pathogenic variants. A number of approaches are being developed to address this issue, including efforts to enhance data and knowledge sharing, high throughput functional assays and quantitative and machine learning variant classification methods. Finally, the more complex and comprehensive genetic basis of inherited cardiac diseases is now being investigated in greater depth, in particular though genome-wide association studies utilising multicentre studies and population biobanks. This research offers the promise in the near future of more comprehensive diagnostic sequencing that may more accurately predict the risk of disease onset, severity and outcomes in patients and family members.

R. Walsh: None.

\section{S08.3}

\section{Identification and Characterization of Novel Micropro-} teins in the Human Heart

\section{S. van Heesch*}

Max-Delbrück-Centrum for Molecular Medicine in the Helmholtz Association (MDC), Berlin, Germany

RNA translation is widespread outside of the canonical protein-coding complement of the human transcriptome, vastly expanding its coding potential. In a cohort of 80 human hearts, we have recently identified hundreds of previously undiscovered microproteins translated from upstream open reading frames (uORFs) in mRNAs and short ORFs (sORFs) in presumed long noncoding RNAs (lncRNAs) (van Heesch et al., Cell 2019). Although these included translated lncRNAs with known functional roles in the heart (e.g. UPPERHAND, Myheart, Chaer, TRDN-AS and GATA6-AS1), the biological activity of the microproteins produced from these genes remained uncertain.

To address this, we characterized the subcellular localization, cell type-specific expression, and protein interactomes of hundreds of cardiac microproteins in a high-throughput manner. Many of the investigated 
microproteins (57 out of 153 tested) localized to the mitochondria, suggesting underappreciated functionality in cardiac energy metabolism. Using a PRISMA screen, for which tiled 15-aa segments of 271 novel microproteins were spotted on a membrane and incubated with cell lysate, we catalogued microprotein interaction partners and finemapped precise interaction motifs, allowing us to categorize functional microprotein classes. Among the identified microproteins, 18 were specifically produced in the human heart, most of which were cardiomyocyte-specific in a single nucleus expression atlas with $>500 \mathrm{k}$ sequenced nuclei. Genetic perturbation of one of these human heart specific microproteins primarily affected expression of genes involved in oxidative phosphorylation and calcium handling in hiPSC-derived cardiomyocytes. Preliminary live-cell imaging of TTN-GFP labeled microprotein $^{-1-}$ cells revealed a likely repressive function of this microprotein on sarcomere contraction and relaxation times.

We have identified and characterized hundreds of novel microproteins that are produced in the human heart, though prevalent in other tissues. Despite the mostly evolutionary young age of these microproteins, we discovered dedicated subcellular localizations, highly specific interaction partners, cell-type specificity, and functional involvement in processes central to cardiac functioning.

S. van Heesch: None.

\section{S09 Beyond Mendelian Inheritance}

\section{S09.1}

\section{From digenic combinations to oligogenic networks via a} new predictive approach

\section{T. Lenaerts*}

\section{Université Libre de Bruxelles, Brussels, Belgium}

The wide-spread use of high throughput-sequencing in the last decades has helped immensely in unravelling the genetic architecture of many rare diseases as wells as identifying the causative variant in Mendelian ones. Notwithstanding the many successes, different issues remain unsolved: Certain cases exhibit incomplete penetrance, phenotypic variability, locus heterogeneity, as well as non-Mendelian patterns of inheritance. Novel technological advancements are thus required to move beyond this current state of the art. Our team pushes the envelope on new pathogenicity prediction and ranking tools for the identification of the oligogenic causes of diseases. We aim to identify more complex inheritance models, where variants in a small number of genes cause or modulate the development of disease. In this presentation I discuss two advancements we recently published: a new pathogenicity predictor, developed using published medical data on digenic diseases, and an online, publicly available platform that takes the results of this predictor, aggregates this into oligogenic networks, which combine highly affected gene pairs, and contextualises these results with molecular knowledge. This platform, called the Oligogenic resource for variant analysis (or ORVAL), allows researchers to perform predictions on gene panels as well as full exomes, receiving a full report on the potentially relevant combinations, integrated into gene-pairs and networks, and annotated with known protein relationships as well as functional information for all candidate pathogenic combinations that can be found. These two advancements provide researchers with a novel way to explore patient exome data while also adding molecular knowledge and other pieces of information. The predictor, which is called the variant combination pathogenicity predictor (or VarCoPP) assigns pathogenicity scores to variant combinations in gene pairs, similarly to well-known monogenic pathogenicity predictors currently used in prioritisation pipelines. Yet, as VarCoPP is trained on digenic diseases data, it identifies other culprits than those that would be identified in a monogenic way, opening new routes for exploration when the classical approaches fail. In this way, we hope to assist in providing more accurate insights into the reasons for phenotypic heterogeneity in diagnosed patients as well as to raise the diagnosis level for many rare diseases.

T. Lenaerts: None.

S09.3

Resolution of Disease Phenotypes Resulting from Multilocus Genomic Variation

\section{J. E. Posey ${ }^{*}$}

BCM225, Baylor College of Medicine, Houston, TX, United States

Elucidation of the genetic mechanisms underlying disease is a critical step toward Genomic Medicine. As our understanding of the role and frequency of multilocus genomic variation has grown, it is increasingly clear that the relationships between an individual's genotype and phenotypic expression are complex, and that such cases of multilocus variation are challenging to ascertain clinically. We now know that least $5 \%$ of individuals for whom clinical exome sequencing (cES) is diagnostic are identified to have more than one molecular diagnosis, and the rate of multiple molecular diagnoses is increasing with continued novel disease gene discovery. Population substructure - identity-by-descent - can drive an even higher frequency of multilocus genomic variation: $22.0 \%(18 / 82)$ of cases compared to a control cohort with a $4.9 \%(101 / 2076)$ frequency of multilocus variation. We find that the observed rate of multiple molecular diagnoses is 
lower than that predicted, suggesting that such cases may be under-ascertained. In seeking approaches to improve ascertainment of multilocus variation, we find that the observation of apparent phenotypic expansion in association with a previously-described gene may actually represent unrecognized multilocus variation. ES analysis of cases with apparent phenotypic expansion reveals a higher frequency of multiple molecular diagnoses (31.6\% [6/19]) compared to a cohort of cases without apparent phenotypic expansion (2.3\% [2/87, p $=0.0004]$ ), providing potential molecular mechanisms for the observed clinical phenotypes in such cases. Applying a computational approach to model genotype-phenotype relationships in the setting of multilocus variation, we demonstrate that phenotypic similarity analyses can be used to model pairs of Mendelian conditions having distinct or overlapping phenotypic features $\left(\mathrm{p}=1.77 \times 10^{-7}\right)$. Preliminary data suggest that a 'goodness-of-fit' approach to modeling of patient phenotypes and identified molecular diagnoses may support identification of cases likely to have additional molecular diagnoses. Computational modeling of observed and expected clinical phenotypes will play an increasing role in human genomics as our understanding of the combinatorial effects of variation at more than one locus increases in complexity.

J. E. Posey: None.

\section{S10 Single Cell Cancer Genetics}

\section{S10.1}

\section{Heterogeneity}

\section{B. Thienpont ${ }^{*}$, M. De Borre, P. Van Minsel, Q. Yu, E. Galle}

\section{KU Leuven, Laboratory for Functional Epigenetics, Leuven, Belgium}

Lung tumours are heterogeneous, being composed of a number of stromal cell types as well as cancer cells. In this talk, I will describe our recent efforts to unravel the heterogeneity of stromal cells within the lung tumour microenvironment, and describe the ongoing studies in my lab aimed at understanding how epigenetic enzymes can drive transcriptional heterogeneity within cancer cell populations.

B. Thienpont: None. M. De Borre: None. P. Van Minsel: None. Q. Yu: None. E. Galle: None.

\section{S10.3}

Single cell to understand AML progression

\section{A. Perl $^{*}$}

Philadelphia, PA, United States
Acute myeloid leukemia (AML) is a highly studied cancer in which tumor cells have a small number of recurrent, welldescribed somatic mutations in a limited number of functional classes of genes. Whole genomic sequencing of AML tumor cells confirmed these tumors to be relatively simple genetically in individual cells, yet patients treated with molecularly-targeted therapy often relapse with highly polyclonal disease, including genotypes rarely if ever seen outside of this context.

We sought to study and characterize this polyclonality using single cell techniques in order to better understand molecular underpinnings of drug resistance and perhaps identify clinical approaches that would circumvent these. This talk will describe the genetics of AML and describe how single cell analysis for the majority of recurrent mutations seen in AML uncovered unexpected genetic diversity and provided insight into intratumor heterogeneity with implications for targeted therapy of other malignancies.

\section{S11 Embryo and Placenta: Equal Partners}

S11.1

The unsung hero: The essential role of the placenta for development and lifelong health

\section{Hemberger ${ }^{1,2,3 *}$}

${ }^{1}$ Calgary, AB, Canada, ${ }^{2}$ University of Calgary, Calgary, $A B$, Canada, ${ }^{3}$ Alberta Children's Hospital Research Institute, Calgary, AB, Canada

\section{The unsung hero: The essential role of the placenta for} development and lifelong health

Despite significant progress over the past decades in gaining a better understanding of placental development and the contribution of the placenta to fetal and postnatal health, the placenta still remains a much too often overlooked organ. Here, we present data from a recent high-throughput phenotyping screen in which we systematically analysed the placental morphology of mouse mutants that cause embryonic lethality. Much in contrast to the known $\sim 10 \%$ of extra-embryonic defects reported for gene deletions that lead to intra-uterine demise, our systematic analysis shows that indeed $60-70 \%$ of these mutants are associated with a placental phenotype. This discrepancy results from the fact that the placenta is frequently ignored in the analysis of gene mutations, and demonstrates a great gap in knowledge as to the cohort of genes required for normal placentation. Linking the placental phenotype data with embryonic phenotypes obtained from the very same mouse lines, we observe a strong correlation between defects in placental development and abnormalities in heart, brain and vascular development. 
These unbiased correlations underpin the importance of the placenta not just merely for fetal nutrition, but for embryonic development on the whole which has longlasting consequences for postnatal health. In addition to genetic mutations, pregnancy complications that are unrelated to karyotypic abnormalities but often have their roots in a defective placenta are well known to become more prevalent with advanced maternal age. Here, we also present data on the impact of maternal age on placentation, and how changes to the uterine epigenome that accumulate with age contribute to these defects.

\section{S11.2}

Genetics of Recurrent Hydatidiform Moles: Novel Findings and Patient Counselling

\section{R. Slim*}

\section{McGill University Health Centre Research Institute,} Montreal, QC, Canada

Hydatidiform mole (HM) is a human conception with abnormal embryonic development and excessive trophoblastic proliferation. The common form of this condition is sporadic, not recurrent and affects one in every 600 pregnancies in western countries. HM are classified according to their morphological features (complete and partial) and genotypes (Diploid androgenetic, monospermic and dispermic; triploid dispermic, and diploid biparental). Recurrent HM affect for 1 to $9 \%$ of women with a prior HM and may have any of the three genotypes. Biallelic mutations in two genes, NLRP7 and KHDC3L, underlie most cases of recurrent diploid biparental moles. Recently, we identified three novel genes, MEI, TOP6BL and REC114, responsible for androgenetic $\mathrm{HM}$ associated with recurrent miscarriages and with female and male infertility. We demonstrated that HM tissues from two patients with recessive MEII mutations are diploid androgenetic monospermic. Androgenetic HM were first described in 1977, but how they occur have remained open questions. We next investigated the occurrence of androgenesis in Meil-deficient female mice and discovered that $8 \%$ of their oocytes lose all their chromosomes by extruding them with the spindles into the first polar body. We demonstrated that $\mathrm{Meil}^{-1-}$ oocytes are capable of fertilization and 5\% of them produce androgenetic zygotes. We therefore uncovered a novel meiotic abnormality in mammals and a mechanism for the genesis of androgenetic zygotes. Our findings will advance our understanding of the genetic causes of reproductive failure in humans and improve patient management and genetic counselling.

R. Slim: None.

\section{S11.3}

Organoids to understand pregnancy disorders

\section{G. J. Burton*}

\section{University of Cambridge, Cambridge, United Kingdom}

Human early pregnancy is a difficult period to research, yet mounting evidence indicates that many complications of pregnancy, ranging from miscarriage to growth restriction, are the result of poor placental development from implantation onwards. It is now appreciated that development of the placenta is supported predominantly by histotrophic secretions from the uterine glands during the first trimester. These secretions are well recognised in domestic species, where they are generically referred to as 'uterine milk' due to their high lipid content. Research in the sheep and horse has shown how lactogenic hormones secreted by the placenta signal to the glands and upregulate the secretion of 'uterine milk' proteins and growth factors. These in turn feedback on the placenta to stimulate proliferation and differentiation, in what has been referred to as a servo-mechanism.

We have proposed an equivalent mechanism operates in the human, but gaining evidence in vivo is difficult for ethical reasons. We have therefore adopted the organoid approach, generating organoid cultures of both the endometrial glands and the placental trophoblast. These are genetically stable in culture and hormonally responsive, and can be passaged for over one year or frozen for biobanking. The transcript profile of the organoids closely resembles that of the starting material. Exposing our endometrial organoids to various combinations of pregnancy hormones, such as human chorionic gonadotropin and prolactin, upregulates the transcription and secretion of known 'uterine milk' proteins and growth factors that have a mitogenic effect on the placenta.

The organoid approach therefore opens new possibilities for researching the critical events during early placental development in a systematic fashion. Our findings also reinforce the concept that endometrial function and correct decidualisation during the first trimester are critical to pregnancy outcome.

G. J. Burton: None.

\section{S12 Where Science and Ethics Meet}

\section{S12.1}

The state of the science of heritable human genome editing and ethical concerns

\section{Ramsay*}

Sydney Brenner Institute for Molecular Bioscience, Faculty of Health Sciences, University of the Witwatersrand, Johannesburg, South Africa 
Scientific advances in DNA editing technologies are approaching levels of accuracy and efficiency that bring us closer to imagining a responsible clinical translational pathway toward heritable human genome editing (HHGE). This profound step would signal the acceptance of wilful manipulation of human genomes, such that the changes are passed on to future generations. It has triggered the formation of two international panels to debate a responsible way forward. The WHO has convened an Expert Advisory Committee on Human Genome Editing to make recommendations on appropriate governance standards and mechanisms for oversight of somatic and germline human genome editing. The UK Royal Society and US National Academies of Medicine and Sciences convened an International Commission on the Clinical use of Human Germline Genome Editing. The Commission has been tasked with developing a framework to inform the development guidelines for a responsible pathway from research to potential clinical application. This should be done in the context of international discussions of societal implications and include considerations of access, equity and ethical norms and practices. The specific tasks include: Identifying scientific issues that should be evaluated for various classes of HHGE; identifying appropriate protocols to guide pre-clinical validation of potential adverse effects such as unintended on- and off-target events, mosaicism and long term effects; considering how to assess the balance between potential benefits and harms to the child and future generations; providing guidelines for ethics approval and consent, as well as long-term monitoring of children born with edited genomes; and considering potential oversight structures including the scientific criteria for establishing and monitoring HHGE. The Commission's recommendations should be applicable to the global community and guide national policy development and processes. * Ramsay is a member of the International Commission on the Clinical use of Human Germline Genome Editing

M. Ramsay: None.

\section{S12.2}

\section{Crack down on genomic surveillance}

\section{Y. Moreau*}

University of Leuven, Leuven, Belgium

Biometric surveillance is rapidly reshaping life in societies across the world. Genome sequencing is revolutionizing medicine. At the confluence of these two trends lies forensic DNA profiling, which is touted by law enforcement and governments as a silver bullet against crime. Serious concerns emerge however when this technology becomes a cornerstone of human rights abuse, from Xinjiang in China to the US-Mexico border. Moreover, consumer genomic databases are now putting the privacy of the entire population in jeopardy, and clinical genomic databases might soon as well. Even where legal frameworks protect fundamental rights and privacy, major failures are seen in practice. Furthermore, legal instruments are lacking to hold corporations accountable when they recklessly supply technology to abusive authorities. The academic community and scientific publishers have also failed to uphold their professed ethical standards, and hundreds of ethically questionable papers have been published in mainstream scientific journals. Urgent action is needed to shore up social norms, ethical standards, and legal frameworks to regulate biometric surveillance technology, including DNA profiling.

Y. Moreau: None.

\section{S13 Lysosomes in Health and Disease}

\section{S13.1}

The lysosome in the control of cell metabolism

\section{A. Ballabio ${ }^{1,2,3^{*}}$}

${ }^{I}$ Telethon Institute of Genetics and Medicine (TIGEM), Pozzuoli, Italy, ${ }^{2}$ Neurological Research Institute, Baylor college of Medicine, Hoston, TX, United States, ${ }^{3}$ Casma Therapeutics, Boston, MA, United States

The lysosome is the main mediator of cellular degradation and recycling processes. Several diseases are due to lysosomal malfunction. We have identified a lysosomal gene network and a master gene, TFEB, that controls lysosomal biogenesis and autophagy. The activity of TFEB is regulated by the mTORC1 kinase complex through a lysosomal signaling pathway that enables lysosomal function to adapt to environmental cues, such as nutrient availability. Thus, the lysosome acts as a signaling hub to control cell homeostasis and the switch between anabolism and catabolism. Targeting lysosome-mediated signaling pathways is an attractive therapeutic strategy for a variety of disease conditions.

A. Ballabio: Other; Significant; CASMA THERAPEUTICS.

\section{S14 ESHG-ASHG Building Bridges Session on Polygenic Risk Scores: Risky or Useful in the Clinic?}

\section{S14.2}

Improving Polygenic Risk Scores,but also improving our knowledge about them

K. Fischer ${ }^{*}$, K. Läll, M. Mändul

Tartu University, Tartu, Estonia 
In this presentation, the following aspects of implementation of the polygenic risk score (PGRS) will be discussed. First, we consider one of the main problems in the PGRS development - in many studies, there is some overlap between samples used in the different steps: discovery GWAS, score development and selection of the best PGRS, and finally, validation of the final PGRS. Next, we will highlight that there is no unique solution for choosing the optinal PGRS. Depending on the phenotype, different methodologies may lead to the best solution (simple PGRS that uses clumping of the SNPs in the discovery study and a p-value threshold, a PGRS with modified weights, MetaGRS, LDPRED and other Bayesian approaches, machine-learning methods, etc) A thirg range of problems is connected to the effect size estimation of the PGRS. We will show that incorrect time scale as well as the presence of competing risks may lead to biased and misleading estimates of the effect size. Finally, we address the question on how to assess the added value of the PGRS to the existing risk-prediction algorithms. We show that some results that have been presented with some scepticism may actually deserve another look.

K. Fischer: None. K. Läll: None. M. Mändul: None.

\section{S15 Detection of Structural Variation}

\section{S15.1}

\section{Detection of Structural Variation and Haplotype-Aware Genome Assembly}

\section{T. Marschall}

\section{Heinrich Heine University, Düsseldorf, Germany}

Recent breakthroughs in long-read sequencing technology and assembly methodology enable the de novo assembly of human genome to a quality approaching the quality of the reference genome GRCh38. My talk will center around creating such assemblies and how they facilitate the discovery of structural variation. My talk comes in three parts:

In the first part, I will describe work done by the Human Genome Structural Variation Consortium (HGSVC) and summarize how we performed (reference-based) haplotype phasing followed by local assembly to define the sequences of SV alleles (Chaisson et al., Nature Communications, 2019).

In the second part, I introduce a workflow for fully phased de novo genome assembly, overcoming the prevailing genome assembly paradigm to produce sequences that "collapse" parental haplotypes into a consensus sequence. To achieve this, we leverage the chromosome-wide phasing and scaffolding capabilities of single-cell strand sequencing (Strand-seq) and combine them with either high-fidelity (HiFi) long sequencing reads, PacBio CLR reads, or Oxford
Nanopore Technology reads. Employing this strategy, we produce completely phased de novo genome assemblies separately for each haplotype of a single individual of Puerto Rican origin (HG00733) in the absence of parental data. The assemblies are accurate $(\mathrm{QV}>40)$, highly contiguous (contig N50 > $25 \mathrm{Mbp}$ ) with low switch error rates $(0.17 \%)$ providing fully phased single-nucleotide variants $(\mathrm{SNVs})$, indels, and structural variants $(\mathrm{SVs})$.

In the third part, I sketch a way forward to leverage such haplotype-resolved genome assemblies to build a human pangenome. I present preliminary result on a new tool, PanGenie, able to genotype variation, and particularly SVs, inherent to such a pangenome given a short read data set of a study sample. This process is very scalable, going from input Fastq to final call set in around 20 single core CPU hours, and provides accurate genotypes for many SVs that used to be inaccessible by short reads.

T. Marschall: None.

\section{S15.2}

Improving the detection and genotyping of structural variation

\section{B. $\operatorname{Kehr}^{1,2 *}$}

${ }^{1}$ Berlin Institute of Health (BIH), Berlin, Germany, ${ }^{2}$ Charité - Universitätsmedizin Berlin, Berlin, Germany

Structural variants (SVs) can lead to a variety of diseases, contribute significantly to human genetic diversity, and are important marks of genome evolution. Therefore, incomplete detection and unreliable genotyping of SVs limit a wide range of human genetic studies. As recent studies have demonstrated that the number of SVs per human genome is at least an order of magnitude greater than the number of SVs we routinely detect in short read data, we need to explore opportunities to improve the detection and genotyping of SVs. One such opportunity is the use of data from novel technologies, e.g. long read data. Another opportunity is joint detection of SVs in many genomes together, which strengthens the detection of variants shared by different genomes. In this presentation, I will introduce computational methods for detecting and genotyping SVs in many short-read sequenced genomes jointly and demonstrate how the joint approach leads to more complete and more reliable SV call sets. Specifically, I will give an overview of the tools PopDel and PopIns we developed for identifying deletions and non-reference sequence variants in up to tens of thousands of human genomes. Finally, I will show how even the current SV callers for long read data are limited in detecting certain types of SVs.

B. Kehr: None. 


\section{S15.3}

Annotation and Curation of High-Confidence Structural Variants

\section{A. Quinlan*}

\section{University of Utah, Salt Lake City, UT, United States}

A typical human genome contains $>4,000$ structural variants (SVs) larger than $300 \mathrm{bp}$, ranging in size from hundreds to millions of nucleotides. SVs cause diverse phenotypes, and their size increases the potential to alter gene dosage, alter chromatin structure, affect expression, remove regulatory elements, and create gene fusions. However, detecting structural variants in a human genome via whole-genome sequencing is fraught is notoriously inaccurate. I will present recently developed methods for isolating high-confidence structural variants, recent advances in prioritizing SVs that may have phenotypic consequences, and challenges that remain in interpreting this important, yet complex form of genetic variation.

A. Quinlan: None.

\section{S16 Genetic Mechanisms in Haematological Neoplasias}

\section{S16.1}

\section{Inherited Myeloid Malignancies}

\section{J. Fitzgibbon *}

\section{Queen Mary University of London, London, United Kingdom}

Inherited haematological malignancy syndromes are typically characterised by acute myeloid leukaemia/myelodysplasia (AML/MDS) occurring in two or more genetically related family members. While these syndromes are considered relatively rare, accounting for $<5 \%$ of all AML/ MDS cases, it is likely that many patients with germline mutations are not readily identified, families may display marked variations in disease phenotype, latency and penetrance across mutation carriers and these features, coupled with an absence of comprehensive diagnostic assays and paucity of clinical guidelines, give rise to an apparent under-representation of familial leukaemia. Importantly, lack of awareness regarding familial involvement may lead to the unsuspecting selection of asymptomatic family members as stem cell donors, with several reports describing donor-derived episodes of AML. Fortunately, we are now making significant progress in recognising and understanding the molecular pathogenesis of these syndromes, as highlighted by the introduction of a provisional diagnostic category in the World Health Organisation (WHO) 2016 (Myeloid neoplasms with germ line predisposition). To date mutations in $>20$ discrete loci have been described but notably, investigators still report that perhaps as many as $40-50 \%$ of families with an appropriate clinical history have no readily identifiable genetic lesion from interrogation of the coding genome. Unsurprisingly, there is a significant overlap between genes mutated in both sporadic and inherited forms of leukaemia. Each gene has a distinct clinical, biological and molecular profile; for example, familial CEBPA-mutated AML is a recognized leukaemia predisposition entity where the pathological features and long-term survival of individuals mirrors sporadic CEBPAdm AML. We are lately beginning to decipher the genetic evolution of leukaemia across family members, with evidence suggesting that secondary mutations may cluster within pedigrees, for example GATA2 mutations in families with germline CEBPA mutations and JAK-STAT pathway mutations in RUNXI pedigrees potentially pointing to the host genotype impacting on the acquisition of somatic mutations. In the absence of standardised testing, much of our new knowledge is emerging from individual research groups and collaborations and will require validation and collective evaluation across the leukaemia research community. It is widely accepted that we need to develop a network of international advisors who may provide accessible guidance for clinicians worldwide, particularly when rare and insightful clinical observations may aid both clinical management and promote the detection of novel syndromes. Moving forward, it would be sensible to have an unified approach to analyse sporadic and familial leukaemia is now required.

J. Fitzgibbon: None.

\section{S16.2}

\section{Genetics and Mechanisms of Acute Lymphoblastic} Leukemia

\section{A. Ferrando*}

\section{Columbia University, New York, NY, United States}

Relapse acute lymphoblastic leukemia (ALL) is associated with chemotherapy resistance and poor prognosis. Persistent leukemia initiating cells with increased self-renewal capacity, clonal heterogeneity and selection of resistancedriving genetic alterations have been proposed as drivers of leukemia relapse. Gain of function mutations in the $5^{\prime}$ Nucleotidase, Cytosolic II (NT5C2) gene are selectively present in relapsed ALL and confer resistance to chemotherapy with 6-mercaptopurine (6-MP). Yet, the specific mechanisms mediating constitutive activation of NT5C2 and the role of these mutations as drivers of clonal evolution during leukemia initiation and disease progression remain unknown. Our structure function and crystallographic 
analyses have uncovered new intra-molecular mechanisms of NT5C2 regulation specifically targeted by relapsed leukemiaassociated mutations. In addition, using an in vivo leukemia model we demonstrate a dual role for NT5C2 R367Q, the most frequent relapse-associated NT5C2 allele, as a loss of fitness mutation impairing cell growth in the absence of chemotherapy, and a gain of fitness mutation resulting in disease progression and relapse in the context of 6-MP therapy. These results have important implications for monitoring of leukemia relapse driving clones and for the development of targeted therapies for the treatment of relapsed disease.

A. Ferrando: None.

\section{S17 Cornelia de Lange Syndrome and Cohesinopathies}

\section{S17.1}

\section{Cornelia de Lange Spectrum: does deep phenotyping help?}

F. Ramos $^{1 *}$, A. Latorre-Pellicer ${ }^{1}$, L. Trujillano ${ }^{2}$, M. Gil-Salvador ${ }^{1}$, M. Arnedo ${ }^{1}$, C. Lucía-Campos ${ }^{1}$, R. Antoñanzas-Pérez ${ }^{1}$, B. Puisac ${ }^{1}$, P. Gómez-Puertas ${ }^{3}$, J. Pié ${ }^{1}$

${ }^{1}$ University of Zaragoza, Zaragoza, Spain, ${ }^{2}$ University Hospital "Lozano Blesa", Zaragoza, Spain, ${ }^{3}$ Molecular Biology Center "Severo Ochoa", Madrid, Spain

Cornelia de Lange Syndrome (CdLS, OMIM \#122470, \#300590, \#610759 614701, \#300882) is a multisystemic condition characterized by a recognizable craniofacial phenotype, characteristic limb malformations and wide spectrum of symptoms from different organs and systems. Two main clinical subgroups are currently recognized based on the severity of manifestations: the classic (severe) CdLS and the non-classic CdLS. The first subgroup, the most common ( $\approx 60 \%$ of CdLS cases), includes the patients who have a pathogenic variant in the gene NIPBL. These individuals have a characteristic facial appearance, distal limb reduction defects (oligodactyly in the hands), hirsutism, and moderate to severe growth and psychomotor retardation/ intellectual disability. The non-classic CdLS includes the patients with milder craniofacial phenotypes and clinical manifestations and bearing pathogenic variants in the other four genes associated with the syndrome (SMC1A, SMC3, $H D A C 8$, and $R A D 21$ ). Clinical diagnosis of many of these individuals is often challenging and sometimes very difficult due to the overlapping with several syndromes that share clinical manifestations with CdLS but are caused by other genes that may not be related to the cohesin complex. Deep phenotyping can be defined as the precise and comprehensive analysis of phenotypic anomalies in which the individual components of the phenotype are observed and described in detail. The generalized use of the Human
Phenotype Ontology (HPO) system to annotate clinical manifestations has contributed to standardize the terminology and improve the accuracy of the genotype-phenotype correlations in many dysmorphic síndromes, including CdLS. There are a number of tools currently available to perform quick and efficient deep phenotyping processes and algorithms that are able to accurately identify facial patterns (gestalt) that match with specific dysmorphic known síndromes. These new tools, rather than substitute the clinical skills of the clinician, are intended to help them to diagnose patients who do not fit a recognizable dysmorphic pattern. We have used one of those deep phenotyping tools (Face2Gene ${ }^{\oplus}$ ) to check its diagnostic accuracy in $49 \mathrm{CdLS}$ patients whose clinical diagnosis was confirmed by molecular testing. Thirty-three of them had pathogenic variants in NIPBL, and the remaining in three of the other CdLS genes (SMC1A, HDAC8, and RAD21). Average accuracy was $83.7 \%$, meaning that CdLS was listed as the first choice. In $97.9 \%$ of the cases analyzed, CdLS was included in the top-five suggestions. In the subgroup of patients with the classic phenotype, $88.8 \%$ of the patients were diagnosed as CdLS as the first choice. Overall, KBG was the second most suggested diagnosis. No differences were found in the top-one sensitivity when the phenotypes were compared by age. Finally, we check the accuracy of the process comparing the facial phenotypes of the patients depending on the genes involved. We found, as expected, a top-one sensitivity of $97 \%$ for patients with variants in NIPBL, 66,6\% for $R A D 21,60 \%$ for $H D A C 8$, and $50 \%$ for $S M C 1 A$. We can conclude that, in general, deep phenotyping tools are useful for clinical geneticists and may help them in the diagnostic process of patients with non-recognizable dysmorphic patterns or in syndromes with overlapping phenotypes.

F. Ramos: None. A. Latorre-Pellicer: None. L. Trujillano: None. M. Gil-Salvador: None. M. Arnedo: None. C. LucíaCampos: None. R. Antoñanzas-Pérez: None. B. Puisac: None. P. Gómez-Puertas: None. J. Pié: None.

\section{S17.3}

Post-Zygotic Mutation as a Cause of Typical Cornelia De Lange Syndrome

\section{FitzPatrick ${ }^{*}$}

MRC Human Genetics Unit, University of Edinburgh, Edinburgh, United Kingdom

Typical Cornelia de Lange syndrome (CdLS, MIM \#122470) is a very severe, clinically distinct multisystem disorder characterised by intellectual disability, prenatal onset growth retardation, limb malformations, multiple chronic medical problems and distinctive facial features. $60-70 \%$ of affected individuals have de novo heterozygous, loss-of-function 
mutations in NIPBL which are detectable in blood DNA. Many of the remaining typical cases have mosaic mutations in NIPBL usually absent in DNA derived from peripheral leucocytes but detectable in saliva samples at low levels and in uncultured skin with variant allele frequencies approaching $50 \%$. Strikingly most of the children with mosaicism have a clinical disorder at least as severe as those with constitutional mutations. Mosaicism may be the result of post zygotic mutation (PZM) or rescue of a constitutional mutations via mitotic homologous recombination in blood cell progenitors. NIPBL performs fundamental functions critical for cell survival such as mitotic segregation of chromosomes, DNA repair and developmental gene regulation. As such rescue seemed the most plausible mechanism a priori. However using data from whole genome sequence and tissue specific genetic analysis we can find no evidence for rescue and, in a number of cases, definitive evidence for PZM. Given the severity of the phenotype in mosaic cases both at a cellular and organismal level it seems likely that the mutant cells have an advantage over wild-type cells in populating ectodermal neuroectodermal, in particular - lineages. This hypothesis can be tested using in vitro differentiation cell competition assays.

D. FitzPatrick: None.

\section{S18 Clonal Fitness of Somatic Mutations}

\section{S18.1}

\section{Somatic Evolution in Human Tissues}

\section{Martincorena*}

\section{Wellcome Sanger Institute, Cambridge, United Kingdom}

Cancers develop by somatic mutation and clonal selection within our tissues. Over the past decade, the ability to sequence cancer genomes has transformed our understanding of the genetics and evolution of a wide range of cancers. However, owing to technical limitations, little is known about the earliest steps of cancer and how cells in our tissues accumulate mutations during normal ageing and in their progression towards cancer. In this talk, I will present our work unveiling a hidden and unexpected world of cellular competition and somatic evolution in human healthy tissues, with implications for our understanding of cancer and ageing.

I. Martincorena: None.

\section{S18.2}

\section{Somatic Genetic Rescue in Mendelian Haematopoietic Diseases}

$$
\text { P. } \operatorname{Revy}^{*}
$$

Imagine Institute, Paris, France
Somatic mutations occur spontaneously in normal individuals and accumulate throughout life. These genetic modifications contribute to progressive ageing phenotypes and are directly involved in cancer development. However, a growing number of studies of Mendelian haematopoietic disorders indicate that somatic genetic events called "somatic genetic rescue" can offset the pathogenic effect of germline mutations at the cellular level, leading to genetic mosaicism and, in some cases, resulting in a milder disease phenotype. I will present two examples of somatic genetic rescue that we observed in our lab.

P. Revy: None.

\section{S18.3}

Somatic Mutations and Regeneration in Chronic Liver Disease

\section{H. $\mathbf{Z h u ^ { * }}$}

\section{University of Texas Southwestern, Dallas, TX, United States}

Normal tissues accumulate genetic changes with age, but it is unknown if somatic mutations promote clonal expansion of non-malignant cells in the setting of chronic degenerative diseases. Exome sequencing of diseased liver samples from 82 patients revealed a complex mutational landscape in cirrhosis. Additional ultra-deep sequencing identified recurrent mutations in PKD1, PPARGC1B, KMT2D, and ARID1A. The number and size of mutant clones increased as a function of fibrosis stage and tissue damage. To interrogate the functional impact of mutated genes, a pooled in vivo CRISPR screening approach was established. In agreement with sequencing results, examination of 147 genes again revealed that loss of $\mathrm{Pkd} 1, \mathrm{Kmt} 2 \mathrm{~d}$, and Arid1a promoted clonal expansion. Conditional heterozygous deletion of these genes in mice was also hepatoprotective in injury assays. Premalignant somatic alterations are often viewed through the lens of cancer, but we show that mutations can promote regeneration, likely independent of carcinogenesis.

H. Zhu: E. Ownership Interest (stock, stock options, patent or other intellectual property); Modest; Ionis. F. Consultant/Advisory Board; Modest; 28-7 Therapeutics.

S19 Data-driven Genomic Care and its Implementation in the Clinic: A Paradigm Shift

S19.2

Empirical evidence in preventive genomics: MedSeq, BabySeq and beyond

C. Blout*

Brigham and Women's Hospital, Boston, MA, United States 
As genomic technologies advance and costs decline, the ability to implement preventive genomics into everyday medicine becomes possible. The Genomes2People (G2P) research program is exploring the impact of implementing genomic sequencing into routine medical care. This presentation will summarize G2P studies as well as the development of the new Brigham Preventive Genomics Clinic. The MedSeq Project, which was the first randomized control trial of genome sequencing (GS), enrolled not only a disease cohort (cardiomyopathy), but also a healthy cohort. We enrolled 200 patients and 20 of their health care providers. In the separate but similarly designed BabySeq Project, we enrolled 309 newborns recruited from the well-baby nursery and neonatal intensive care unit. Both of these NIH-funded studies explored the medical, behavioral and economic outcomes of GS following disclosure.

Comprehensive sequencing and interpretation revealed that $20 \%$ of MedSeq, and $11 \%$ of BabySeq, participants had a non-indication-based, unanticipated variant in a gene associated with a monogenic condition. Upon rephenotyping, after the variant was identified, 6/20 MedSeq participants and 11/18 BabySeq participants were identified to have a personal and/or family history of the disease. Carrier variants were identified in $94 \%$ of MedSeq and $88 \%$ of BabySeq participants. In MedSeq there were no significant cost differences between sequenced cases and controls by 6 months, and in BabySeq there was a small but significant increase in downstream health care costs over 3 months among the sequenced infants when compared to the controls. Both studies revealed no increases in anxiety and depression related to genomic information. Lessons learned from this research prompted the creation of the Brigham and Women's Hospital Preventive Genomics clinic. This first of its kind service has seen over 40 clinical patients to date for preventive genomic consultations and testing. Our research outlines the many benefits of population genomic screening and our experiences provide important information regarding clinical implementation as well as identifying areas where further study is needed.

C. Blout: None.

\section{S20 Integrating Large-scale Functional Data Into Genome-Wide Association Studies}

\section{S20.1}

\section{Combining omics data with GWAS results}

\section{A. Gusev ${ }^{1,2 *}$}

${ }^{1}$ Dana-Farber Cancer Institute, Boston, MA, United States, ${ }^{2}$ Harvard Medical School, Boston, MA, United States
Genome-wide association studies (GWAS) have identified thousands of variants associated with disease, but the underlying mechanisms through which these variants operate remain elusive. This talk focuses on three topics related to overcoming this challenge: First, I will discuss some insights into disease architecture that we have gained from GWAS so far. Second, I will describe a statistical framework we have developed called MESC, which aims to quantify the total fraction of disease heritability that can be explained by all observed expression QTLs. Applying MESC to large-scale eQTL and GWAS data we find that only $\sim 11 \%$ of GWAS heritability appears to be mediated by measurable eQTLs, most of which resides in eQTLs with very weak effects that are difficult to detect. Finally, I will describe a computational tool - PLASMA - that leverages allele-specific expression to fine map such weak effect QTLs and colocalize them with disease. In application to real RNA-seq studies from hundreds of individuals, PLASMA increased power over conventional QTL mapping and colocalization by an order of magnitude.

A. Gusev: None.

\section{S20.2}

A Bayesian Framework for Joint Fine-Mapping with Applications to Immune-Mediated Diseases

\section{J. Asimit}

MRC Biostatistics Unit, University of Cambridge, Cambridge, United Kingdom

Hundreds of genetic variants have been identified as associated with a spectrum of diseases and related traits but pinpointing likely causal variants (fine-mapping) has been complicated by extended linkage disequilibrium (LD) and finite sample sizes. In the fine-mapping of single outcomes, inaccuracies often occur when there are two or more distinct causal variants that are both correlated with a single noncausal variant. This motivates the development of MFM (Multinomial Fine-Mapping) for multiple diseases with shared controls; joint fine-mapping that leverages information between outcomes in a Bayesian framework, where prior model probabilities are formulated to favour combinations of models with a degree of sharing of causal variants between outcomes. Our simulation studies demonstrate that MFM has greater accuracy than single disease fine-mapping when there are shared causal genetic variants between diseases, yet no precision loss if there is no sharing. In addition, MFM analysis of six immune-mediated diseases identifies novel genetic associations that are missed by marginal analyses. We jointly fine-map association signals for six diseases and of particular interest is IL2RA, which is known to be associated with several autoimmune 
diseases, including multiple sclerosis (MS), type 1 diabetes (T1D), autoimmune thyroid disease (AITD) and coeliac disease. MFM has the potential to increase fine-mapping resolution in related diseases/traits without collecting larger sample sizes and is computationally efficient.

J. Asimit: None.

\section{Educational Sessions}

\section{E01 New Technologies}

E01.1

Manifold destiny: exploring genetic interactions in high dimensions through massively parallel single cell RNAseq

\section{J. Weissman*}

\section{UCSF/HHMI, San Francisco, CA, United States}

A major principle that has emerged from modern genomic and gene expression studies is that the complexity of cell types in multicellular organisms is driven not by a large increase in gene number but instead by the combinatorial expression of a surprisingly small number of components. This is possible because specific combinations of genes exhibit emergent properties when functioning together, enabling the generation of many diverse cell types and behaviors. Understanding such genetic interactions has important practical and theoretical applications. For example, they can reveal synthetic lethal vulnerabilities in tumors, identify suppressors of inherited and acquired disorders, guide the design of cocktails of genes to drive transdifferentiation between cell types, inform the search for missing inheritance in genetic studies of complex traits, and enable systematic approaches to define gene function in an objective and principled manner. Defining how genes interact is thus a central challenge of the post-genomic era.

The combinatorial explosion of possible genetic interactions (GIs), however, has necessitated the use of scalar interaction readouts (e.g. growth) that conflate diverse outcomes. I will present our work developing an analytical framework for interpreting manifolds constructed from high-dimensional interaction phenotypes. We applied this framework to rich phenotypes obtained by Perturb-seq (single-cell RNA-seq pooled CRISPR screens) profiling of strong GIs mined from a growth-based, gain-of-function GI map. Exploration of this manifold enabled ordering of regulatory pathways, classification of GIs in a principled manner (e.g. identifying true suppressors), and mechanistic elucidation of synthetic lethal GIs, including an unexpected synergy between CBL and CNN1 driving erythroid differentiation. Finally, we applied recommender system machine learning to predict interactions, facilitating exploration of vastly larger GI manifolds. We expect the conceptual and computational frameworks presented here will be broadly applicable to genetic interactions obtained via other rich phenotyping approaches (e.g. proteomics, imaging) and methods of perturbation (e.g. knockdown, knockout, mutagenesis).

J. Weissman: None.

\section{E01.2}

Nanopore Sequencing of Human Genomes: the long and short of it.

\section{Loose*}

School of Life Sciences, University of Nottingham, Nottingham, United Kingdom

The utility of long read sequencing has dramatically increased in the last few years whilst costs have lowered. We have exploited Nanopore sequencing to sequence human genomes (and others) on MinION, GridION and PromethION. To do this we have been involved in developing methods to sequencing ever longer molecules, currently up to $2.3 \mathrm{Mb}$ in length. Alongside we have been developing methods for targeted sequencing of specific regions of genomes using CAS-9 library preparation methods and, more recently, computational methods including adaptive sampling allowing us to direct the sequencer to specific regions of the genome as required. We will present our work including sequencing over 700 cancer gene targets and the MHC domain to $30 \mathrm{x}$ coverage on a single MinION flowcell.

M. Loose: D. Speakers Bureau/Honoraria (speakers bureau, symposia, and expert witness); Modest; Oxford Nanopore Technologies. F. Consultant/Advisory Board; Modest; UK BioBank.

\section{E01.4}

Optical mapping for clinical structural variants detection

\section{A. Hoischen ${ }^{1,2,3 *}$}

${ }^{1}$ Department of Human Genetics, Radboud University Medical Center, Nijmegen, Netherlands, ${ }^{2}$ Radboud Institute of Molecular Life Sciences, Radboud University Medical Center, Nijmegen, Netherlands, ${ }^{3}$ Department of Internal Medicine and Radboud Center for Infectious Diseases (RCI), Radboud University Medical Center, Nijmegen, Netherlands 
Structural variants (SVs) are an important source of genetic variation in the human genome and they are involved in a multitude of human diseases. SVs are enriched in repeatrich regions of the human genome, and several remain undetected by conventional short-read sequencing technologies. Here I will introduce the basics of high-resolution optical mapping that allow the sensitive genome-wide identification of SVs. I shall demonstrate its utility for molecular cytogenetics for constitutional SVs in cases with e.g. germline micro-deletion/-duplication syndromes, inversions and translocations. Recent advances also allow for the sensitive identification of acquired i.e. somatic SVs e.g. in leukemia samples. Finally I will showcase first examples of how optical mapping enables unravelling of hidden $\mathrm{SVs}$ in rare disease cases that remained undetected by classical methods, including MLPA, Sanger sequencing, exome and/or genome sequencing. In summary, I will show how the complementary use of mapping rather than sequencing approaches allow for comprehensive detection of all cytogenetic abnormalities and how this can unmask hidden SVs. I predict that ultra-long read mapping may enable a revolution in 'next generation cytogenetics'.

A. Hoischen: None.

\section{E02 Abstracts Withdrawn}

\section{E03 Pharmacogenomics in the Clinic}

\section{E03.1}

\section{Pharmacogenetics - from bench to drug label}

\section{J. Stingl}

Institute of Clinical Pharmacology, UniversityHospital of the RWTH Aachen, Aachen, Germany

The role of pharmacogenetic diagnostics has changed during the past decades with a more explorative role in the beginning explaining differences in drug metabolism and elimination, further looking at differences in individual drug response and safety, and nowadays developing to a therapy companion diagnostic for indications of drug therapies. With the knowledge on our pharmacogenome, also new methods for genetic diagnostics arose, and large genome analyses now became convenient, easy to handle, and relatively cheap. While in centralized countries like the United States, the development of guidelines and pharmacogenetic information in drug labels is already advanced, in Europe, the diversity of national health systems affects regulation for pharmacogenetic diagnostics. We have now different sorts of pharmacogenetic information in the drug label which could be informative, actionable, or obligatory. The action consequence of this information depends on the section in the label that contains the information. For example, the section on indication would most probably contain information on mandatory pharmacogenetic diagnostics prior to onset of therapy, whereas in the section on adverse drug reactions, mostly informative pharmacogenetic aspects are mentioned about variability in the risk of side effects. Recently, a European referral was opened that should clear the question of a pretherapeutic testing of DPYD variants in therapy with fluoropyrimidines. The outcome of the referral (expected in 2020) could mean that for the first time in Europe, a pharmacogenetic test is mandatory before onset of a common drug therapy (so far, only genetic testing in rare disease therapy is mandatory). Pharmacogenetics is now in the implication phase with the use of pharmacogenetic tests for drug therapy indication, for individualized dosing and for the avoidance of side effects. Depending also on national health systems, genetic tests for therapy decisions are/and will become more and more in use in realworld clinical practice.

J. Stingl: None.

\section{E04 Hypermobility and the Ehlers Danlos Syndromes}

\section{E04.2}

Vascular Presentation of the Ehlers Danlos Syndromes

\section{P. Byers*}

\section{University of Washington, Seattle, WA, United States}

Normal 0 false false false EN-US X-NONE X-NONE /* Style Definitions */ table.MsoNormalTable \{mso-stylename:"Table Normal"; mso-tstyle-rowband-size:0; msotstyle-colband-size:0; mso-style-noshow:yes; mso-stylepriority:99; mso-style-parent:"'; mso-padding-alt:0in 5.4pt 0in 5.4pt; mso-para-margin-top:0in; mso-para-marginright:0in; mso-para-margin-bottom:10.0pt; mso-para-margin-left:0in; line-height:115\%; mso-pagination:widoworphan; font-size:12.0pt; font-family:"Arial","sans-serif";\} Given that many of the types of Ehlers Danlos syndrome (EDS) result from mutations in genes that encode proteins that are components of blood vessels, it should come as no surprise that arterial dissection and rupture can complicate the lives of people with these conditions. The flagship of these conditions is vascular EDS (vEDS), which results from pathogenic variants in COL3Al that encodes type III procollagen - a major component of arterial walls. These events are uncommon in children but prove to the related to the cause of death in most individuals, except for those with haploinsufficiency. One contribution to premature death (median survival of about 50 years) is that vessel wall is friable and, so, difficult to suture; in addition, it is difficult to anticipate dissection and rupture. Aortic and large arterial vessel dissection appears to be an uncommon event in people with classical EDS the most common kinds of 
mutations (COL5A1 haploinsufficiency) as well as those with missense and splicing alterations. The recessively inherited kyphoscoliotic EDS results from pathogenetic variants in $P L O D 1$ that encodes lysyl hydroxylase 1 (LH1), and in FKBP14. These affect the hydroxylation of lysyl residues in collagens that are critical for the formation of tissue stabilizing intermolecular cross links. In both, dissection may occur in late childhood and early adolescence. Surveillance protocols are not yet standardized among groups and there is not yet consensus recommendations for any of these conditions. Surgical intervention can be lifesaving and should not be ignored, and the effectiveness of medical therapies have yet to be clearly demonstrated. The pathway from nucleotide change to pathogenesis remains to be explored but there are hopes that unexpected therapeutic tools may be identified from ongoing studies.

P. Byers: None.

\section{E05 Interpreting Variation Outside of Protein-Coding Sequence}

\section{E05.1}

\section{Functional Characterization and Therapeutic Targeting of Gene Regulatory Elements}

\section{N. Ahituv ${ }^{*}$}

\section{UCSF, San Francisco, CA, United States}

Nucleotide variation in gene regulatory elements is a major determinant of phenotypes including morphological diversity between species, human variation and human disease. Despite continual progress in the cataloging of these elements, little is known about the code and grammatical rules that govern their function. Deciphering the code and their grammatical rules will enable high-resolution mapping of regulatory elements, accurate interpretation of nucleotide variation within them and the design of sequences that can deliver molecules for therapeutic purposes. To this end, we are using massively parallel reporter assays (MPRAs) to simultaneously test the activity of thousands of gene regulatory elements in parallel. By designing MPRAs to learn regulatory grammar or to carry out saturation mutagenesis of every possible nucleotide change in disease causing gene regulatory elements, we are increasing our understanding of the phenotypic consequences of gene regulatory mutations. Regulatory elements can also serve as therapeutic targets. To highlight this role, we used CRISPR/ Cas9 activation (CRISPRa) of regulatory elements to rescue haploinsufficient diseases (having $~ 50 \%$ dosage reduction due to having only one functional allele) in vivo. By targeting the Siml promoter or its $270 \mathrm{~kb}$ distant hypothalamic enhancer, we were able to rescue the haploinsufficient obesity phenotype in Siml heterozygous mice, both using a transgenic and adeno- associated virus (AAV) approach. Using a similar AAV CRISPRa approach, we also show that we can rescue the obesity phenotype in $M c 4 r$ heterozygous mice. Our results highlight how regulatory elements could be used as therapeutic targets to treat numerous altered gene dosage diseases.

N. Ahituv: E. Ownership Interest (stock, stock options, patent or other intellectual property); Modest; Encoded Therapeutics. E. Ownership Interest (stock, stock options, patent or other intellectual property); Significant; Enhancer Therapeutics.

\section{E05.2}

\section{Dissecting Non-Coding Sequences in Gene Regulation}

\section{A. Stark ${ }^{*}$}

Research Institute of Molecular Pathology (IMP), Vienna, Austria, Vienna, Austria

The vast majority of disease-associated SNPs in the human genome is located in the non-protein-coding part, which makes it difficult to assess the functional impact of the sequence variants. Non-coding sequences, which make up the largest part of the human genome, contain gene-regulatory elements - promoters and enhancers - that direct the dynamic expression of genes in complex spatial and temporal patterns. The promoter and enhancer sequences contain this gene regulatory information, which in the cells is realized by transcription factor and cofactor proteins.

Today, I will first introduce how reporter systems can be used to understand the sequence-encoding of gene-regulatory information and how sequence changes alter regulatory function. I will then present our recent work towards understanding how cells utilize this information with transcription factor and cofactor proteins. Using an interdisciplinary approach in Drosophila, we study how enhancers and the cofactor proteins they recruit activate different types of core promoters, enabling distinct sets of genes and alternative promoters of the same genes to be regulated differently. The distinct compatibilities between cofactors and core promoters forms the basis of specificity within and between gene regulatory programs.

A. Stark: None.

\section{E06 Brain Size Matters!}

E06.1

Microcephaly: genetics and disease mechanisms

G. M. S. Mancini*

ErasmusMC, Department of Clinical Genetics, Rotterdam, Netherlands 
Microcephaly is defined by a head circumference (HC) below 2 SD standard deviations for age and sex. A distinction is generally made between congenital or primary microcephaly, i.e. a small HC for gestational age at birth, and progressive or post-natal microcephaly, i.e. normal HC at birth followed by insufficient growth during infancy and childhood. When small head circumference reflects an insufficient brain growth, microcephaly is associated with neurodevelopmental disorders (NDD), such as intellectual disability, epilepsy, autism. The most common structural abnormality in primary microcephaly is a simplified gyral pattern of the cortex. Association with other malformations, e.g. lissencephaly or polymicrogyria, implies neurological sequelae and influences disease course. Nevertheless, prognostic evaluation in a newborn with microcephaly remains difficult. Compatibly with a very high mitotic rate and scheduled cell death of neural progenitors of the developing human cortex, the most relevant genes and processes affected in primary microcephaly relate to regulation of the cell cycle (e.g. mitotic spindle formation and orientation, centrosome biology, mitotic chromosome structure, double strand DNA repair and chromosome stability) or of scheduled apoptosis. There is partial etiologic overlap with the pathogenesis of progressive microcephaly, which mostly affect control of DNA repair, or regulation of intermediate metabolism. However, novel paradigms are emerging in the pathogenesis of microcephaly, which are related to regulation of the balance between ER-controlled proteostasis and cell cycle. This subtle balance might reveal to be one of the factors determining the difference between a stable microcephaly phenotype (as observed in many types of primary microcephaly) to a progressive, hence severe course as observed in many types of progressive microcephaly.

G. M. S. Mancini: None.

\section{E06.2}

\section{Macrocephaly}

G. Mirzaa ${ }^{1 *}$, F. Pirozzi ${ }^{2}$, A. Timms ${ }^{2}$, M. Berkseth ${ }^{2}, R$. Shear $^{2}$, G. Ruggeri ${ }^{2}$, E. Novotny ${ }^{3}$, W. Dobyns ${ }^{2}$, R. Kapur ${ }^{3}$, J. Hauptman ${ }^{2}$, J. Ojemann ${ }^{2}$

${ }^{1}$ Seattle, WA, United States, ${ }^{2}$ Seattle Children's Research Institute, Seattle, WA, United States, ${ }^{3}$ Seattle Children's Hospital, Seattle, WA, United States

\section{Megalencephaly: advances from genomic discoveries to precision-based medicine}

Megalencephaly disorders (MEG) constitute a growing spectrum of developmental disorders associated with early brain overgrowth, brain malformations including cortical dysplasia, and neurodevelopmental co-morbidities such as intractable epilepsy, autism and intellectual disability. Constitutional and mosaic mutations of multiple cell growth pathways, including the PI3K-AKT-MTOR and RAS pathways, have been identified in this spectrum; with promising therapeutic avenues on the horizon to ameliorate the associated neurological deficits (e.g. MTOR pathway inhibitors). However, significant molecular and clinical challenges remain. Widely used molecular diagnostic methods, such as standard-depth exome sequencing, lack the sensitivity to detect low-level mosaic mutations common in MEG. These methods are also not suitable for screening limited amounts of surgically resected brain tissues due to their high DNA input requirements. Ultimately, more optimal molecular diagnostic methods and high throughput pre-clinical models are necessary to identify and study the effects of MEG-related mutations on brain development. To address these challenges, we utilize a combination of ultra-deep targeted sequencing employing digital droplet PCR (ddPCR) as a new and sensitive tool for screening common mutations in the PI3K-AKT-MTOR pathway, and a quad based exome approach, sequencing lesional (affected) tissues at a higher depth (200-250X). To date, we performed genetic testing on a large cohort of MEG families and have identified both known and novel candidate genetic variants, including ultra-low-level mosaic mutations in the PI3K-AKT-MTOR pathway. Our data describe a gradient of mosaicism seen within the brain in hemimegalencephaly samples; with mutation-positive regions ranging in alternate allele frequencies (AAF) from $0.3 \%$ to $25.6 \%$ across several brain tissues. We subsequently aimed to model disease pathogenesis in vitro, selecting patient-derived fibroblast lines with mutations in key upstream (PIK3CA) and downstream (MTOR) nodes in the pathway. We generated induced pluripotent stem cells (iPSCs) carrying the common PIK3CA $A^{\mathrm{H} 1047 \mathrm{R}}$ and $M_{T O R}{ }^{\text {T1977I }}$ mutations to characterize these nodes. Our results suggest that targeted molecular analysis for the common PI3K-AKT-MTOR pathway mutations by ddPCR is an effective molecular diagnostic approach for MEG. Given the high sensitivity and low DNA input requirements for this assay, ddPCR may also be an effective molecular tool for other mosaic disorders with a narrow mutational spectrum. Our iPSC-derived lines provide a valuable model for dissecting the functional consequences of these mutations and future drug screens using PI3K-AKT-MTOR pathway inhibitors.

F. Pirozzi: None. A. Timms: None. M. Berkseth: None. R. Shear: None. G. Ruggeri: None. E. Novotny: None. W. Dobyns: None. R. Kapur: None. J. Hauptman: None. J. Ojemann: None. 
E07 Genome Editing: Multiple Tools and their Applications

\section{E07.1}

\section{Editing the Mammalian Genome: Targets and Tools}

\section{H. O’Neill*}

\section{University College London, London, United Kingdom}

Genome editing comprises a powerful set of tools which have enabled discovery in every model organism. CRISPR genome editing has propelled research from bench to clinic and shows promise for both the treatment and prevention of genetic disorders. Ongoing research into the components of this technology highlight where improvements can be made to sharpen editing efficiencies. This lecture explains what CRISPR is, how it works, and dissects the CRISPR machinery to illuminate aspects of experimental design which are essential for any genome editing experiment.

H. O’Neill: None.

\section{E07.2}

\section{Genome-Wide CRISPR-Cas9 Screening in Immune} Cells

\section{P. Tolar ${ }^{*}$}

\section{Francis Crick Institute, London, United Kingdom}

The advent of CRISPR/Cas9-mediated genome editing has revolutionised genetics. One of the new experimental approaches afforded by the high efficiency of CRISPR/Cas9 gene editing are high-throughput gene targeting experiments, such as genome-wide functional screens. Pooledlibrary genome-wide screens are a particularly powerful and efficient method to identify non-redundant genes regulating a variety of cell intrinsic functions. For the first time this allows comprehensive molecular characterisation of cellular processes in cells from higher organisms in which previous approaches failed, such as in primary immune cells from mice and humans. I will discuss examples of CRISPR screening strategies that my lab has developed for screening of immune functions in B cells. The results of these screens shed new light on molecular pathways that control antigenmediated B cell activation, proliferation, cell death and differentiation into antibody-secreting cells under healthy and pathological situations. The encouraging results of these approaches hold promise for future advances in studies of gene interactions, pathway mapping and molecular bases of pathology inferred directly from diseased human cells.

P. Tolar: None.
E08 Does Genetic Counselling Work?

\section{E08.1}

What Was, What Is, What If - Trends and Predictions In Assessing Genetic Counseling

\section{R. G. Resta*}

\author{
Swedish Medical Center, Seattle, WA, United States
}

Genetic counselling as a formalized clinical practice is about 75 years old and as a profession is about 50 years old. Initially (What Was) the criteria for assessing the effectiveness of genetic counselling focused on reproductive outcomes, cost-savings, and avoiding disability. As genetic technologies have advanced, social values have changed, and research sophistication has increased, (What Is) the effectiveness of genetic counselling has been assessed by patient focused outcomes such as empowerment, adaptation, and personal disease risk reduction and avoidance. Nonetheless, recurring themes of cost-savings and disability avoidance continue to be proposed and used as measures of genetic counselling success. Ultimately, the measures of the "success" of genetic counselling reflect social, ethical, cultural, geographic, and historical factors, as well as who is measuring genetic counselling outcomes and for what purposes. As genetic technologies and ethical values evolve (What If), different measures of effective genetic counselling will likely emerge.

This presentation reviews the different measures of the effectiveness of genetic counselling and their relationship to sociocultural and historical influences and speculates on emerging, potential, and speculative ways of assessing the effectiveness of genetic counselling. Ultimately, the "effectiveness" of genetic counselling depends upon how effectiveness is defined, who is defining effectiveness, and why they are defining effectiveness. The effectiveness of genetic counselling is defined not so much by its practitioners as it is by the sociocultural, medical, and historical context in which genetic counselling takes place.

R. G. Resta: None.

\section{E08.2}

How to help society interact with genomic technology

\section{A. Middleton ${ }^{*}$}

\section{Wellcome Genome Campus, Cambridge, United Kingdom}

The 'Your DNA, Your Say' project is a global online survey gathering public attitudes towards genomic data sharing. This project explores how society is responding to genomic technology and articulates the hopes and concerns that 
individuals have. I will share results from 37,000 members of the public, from 22 countries (survey gathered data 15 languages) and offer insight into awareness of genomics, interest in donating one's data for research and clinical use and fears around what would happen if a person is identified from their data. I will also explore what trust looks like to members of the public. I was also share details of another study, 'Music of Life' that explores how to explain genetics using metaphor in film. Films are being trialled at Addenbrookes Hospital in Cambridge and I will share some of these. All of my work focusses on how to build a bridge to society so that we can help society interact with genomics. Twitter: @genomethics, website: genomethics.org

A. Middleton: F. Consultant/Advisory Board; Modest; RareMark, Congenica.

\section{E10 Therapy for Rare Disorders}

\section{E10.1}

\section{Gene therapy in genetic disorders}

\section{Cavazzana ${ }^{1,2,3,4,5 *}$}

${ }^{1}$ Assistance Publique - Hôpitaux de Paris, Paris, France, ${ }^{2}$ Biotherapy Department, Hôpital Necker-Enfants malades, Paris, France, ${ }^{3}$ Biotherapy Clinical Investigation Center, Groupe Hospitalier Universitaire Ouest, Paris, France, ${ }^{4}$ Paris Descartes-Sorbonne Paris Cité University, Imagine Institute, Paris, France, ${ }^{5}$ INSERM UMR 1163, Laboratory of Human Lymphohematopoiesis, Paris, France

Over the last fifteen years, gene therapy has shown its powerful outcome to successfully treat genetic diseases such as X-SCID. Moreover the results of several other trials have confirmed the clinical potential of gene therapy approaches in other settings such as ADA deficiency and bhemoglobinopathies where the bone marrow content of the different stem and precursor cells and the cells' relationship with the stroma have very specific characteristics. The optimization of gene therapy requires better characterization or identification of bone marrow homeostasis features in disease settings. Recent progress has been achieved in the harvesting and expansion of healthy hematopoietic stem and progenitor cells (HSPCs) but also in identifying the appropriate tools. The use of SIN retroviral vectors has significantly reduced the risk of insertional mutagenesis and has been the best choice for the introduction of a therapeutic gene into autologous HSPCs than first-generation gamma retroviral vectors. The use of autologous gene-corrected HSPCs reduced the risk of alloreactive immune responses and decreases the risk of infectious complications. Although this progress is of great value, caution is required when translating these findings into a diseased HSPC setting: (i) in order to allow an engraftment of the corrected cells, genemodified autologous HSPCs need a conditioning regimen that could be responsible for acute and chronic toxicity, (ii) HSPCs derived from children under the age of 10 behave differently in culture. Lastly, genome editing and homologous recombination technologies have undergone spectacular developments over the last couple of decades. In view of the impressive progress reported for the gene addition strategy, offering gene-editing approaches to patients affected by inherited disorders would move gene therapy one step forward but would raise a number of other scientific questions.

M. Cavazzana: None.

E10.2

Trial readiness, trial endpoints and stakeholder collaboration

\section{Straub ${ }^{*}$}

Institute of Genetic Medicine, Newcastle University, Newcastle, United Kingdom

Neuromuscular diseases (NMD) are rare diseases caused by abnormalities in four anatomical structures: 1) the anterior horn cell, 2) the axon of the $2^{\text {nd }}$ motor neuron, 3) the neuromuscular junction and 4) the skeletal muscle cell itself. Mutations in more than 600 genes causing 1000 different diseases have so far been identified to cause genetic NMD. The spectrum of phenotypes reaches from floppy babies to late onset degenerative diseases. Most NMD are associated with profound disability and many have a reduced life expectancy. The clinical approach to NMD has changed quite dramatically over the past 30 years from a rather nihilistic and paternalistic to a research active and forward looking approach. Life changing treatments have now been approved for a number of genetic NMD, including gene replacement therapy for spinal muscular atrophy type 1 , and many interventional trials and registration studies are ongoing. This change in attitude was helped by improvements in diagnostics, a better understanding of pathomechanisms and joint international collaborations on trial readiness. Within the past 15 years, a field with a profound lack in clinical trial culture has changed into a specialty that is at the forefront of advanced therapy activities. This change was amongst other things made possible by TREAT-NMD, a patient centred collaborative infrastructure programme supported by patient advocacy groups and the European Commission. The project started as a network of excellence within the EU Framework Program 6 with the aim to accelerate the elaboration of clinical trials for rare genetic NMD. Over the past 13 years the network has established standardised operating procedures for the assessment of various animal models, standard 
of care guidelines for a number of NMD, patient registries, a care and clinical trial site inventory, a registry for outcome measures, the TREAT-NMD advisory committee for therapeutics (TACT), a global communication programme, training and education activities, a consortium of biobanks and close interactions with the regulatory authorities. The successful translational infrastructure has helped to overcome the fragmentation that existed in the field and shaped the way all stakeholders are working together. The presentation reflects on the achievements of TREAT-NMD and on lessons learned.

V. Straub: None.

\section{E11 Heritability Estimations}

E11.1

Heritability estimation and gene-environment
correlation

\section{A. Young ${ }^{*}$}

\section{University of Southern California, Los Angeles, CA, United} States

Heritability measures the fraction of trait variation in a population due to genetic inheritance. However, when genetic variants and environment are correlated, heritability becomes harder to measure. Sources of gene-environment correlation include population stratification and indirect genetic effects from relatives. indirect genetic effects are effects of genetic variants in one individual on another individual's trait through the environment. indirect genetic effects have been shown to be substantial for educational attainment, and likely affect other human traits. In this talk, I discuss how to use genetic data on families to separate out the direct causal effects of inherited genetic variants from indirect genetic effects and population stratification. The particular genetic variant that an individual inherits is the outcome of random segregation in the parent of that individual during meiosis. This randomisation, independent of environment, enables unbiased estimation of direct effects of SNPs and heritability by using genetic data on parents and offspring. We discuss results of applying such methods and compare them to results from methods that do not account for gene environment correlation.

A. Young: None.

\section{E11.2}

Flexible Heritability Estimation from Summary
Statistics

D. Speed ${ }^{*}$

Aarhus Institute of Advanced Studies, Aarhus, Denmark
The field of SNP-based heritability analysis began in 2010, when Yang et al showed it was possible to estimate SNP heritability, the total proportion of phenotypic variation explained by single nuclotide polymorphisms, from genome-wide association study (GWAS) data. Since then, many related methods have been proposed (e.g, methods for estimating heritability enrichments, genetic correlations and confounding bias), that allow us to further interrogate the genetic architecture of complex traits.

I will begin by providing an introduction to the field, and describing methods that use individual-level data. Then I will discuss the second-generation methods, that require only GWAS summary-statistics (and thus allow us to analyze ever-increasing amounts of data). I will explain how a crucial feature of all methods is the choice of heritability model, which reflects our prior information about the relative contributions of different SNPs. I will finish by providing examples not only of how these methods have improved our understanding of complex traits (e.g., allowed us to determine which types of SNPs are most important, and to infer the impact of selection), but how this information can be utilized in other areas of statistical genetics (e.g., to enable us to produce more accurate polygenic risk scores).

D. Speed: None.

\section{E12 Abstracts Withdrawn}

\section{E13 Mainstreaming Mitochondrial Replacement Techniques?}

\section{E13.1}

Mitochondrial replacement techniques: Exploring the biological consequences

\section{Herbert*, G. Hudson, Y. Takeda}

Newcastle University, Newcastle upon Tyne, United Kingdom

Mutations in mitochondrial DNA (mtDNA) are maternally inherited and can cause a broad range of debilitating and fatal diseases. Reproductive technologies, commonly known as "mitochondrial replacement" (MR) have been developed to enable women who carry pathogenic mutations to have a genetically related child with a greatly reduced risk of transmitting mtDNA disease. Such technologies involve transplantation of the nuclear DNA from the egg of an affected woman to an enucleated egg from an unaffected donor, effectively rescuing the nuclear genome from its defective mitochondrial environment. The nuclear DNA is transferred in a karyoplast containing a minimal amount of cytoplasm, which can contain mitochondria. 
Under optimal conditions, the fraction of mtDNA cotransferred with the nuclear genome typically accounts for $0-2 \%$ of the mtDNA content of MR embryos. Despite this, a minority of embryonic stem (ES) cell lines derived from MR embryos revert to the nuclear donor's mitochondrial genome. While the relevance to development in vivo is unclear, reversion in ES cell lines remains a consideration in the therapeutic application of MR. Our ongoing research, focussed on understanding the drivers of reversion and on developing techniques to prevent it, has revealed new insights into mitochondrial homeostasis during preimplantation development.

M. Herbert: None. G. Hudson: None. Y. Takeda: None.

\section{E14 Repeat Expansions: From Detection to Treatment}

\section{E14.2}

Therapeutic advances in Huntington's disease with relevance to other repeat expansion disorders

\section{S. J. Tabrizi*}

\section{University College London, London, United Kingdom}

Huntington's disease (HD) is one of the CAG repeat expansion disorders, it is a devastating autosomal dominantly inherited neurodegenerative disease for which there is currently no effective disease modifying therapy. The genetic predictability of HD provides an opportunity for early therapeutic intervention many years before overt symptom onset and at a time when reversal or prevention of neural dysfunction may still be possible. As HD is monogenic, fully penetrant, and characterised by a long premanifest phase, it is emerging as a potential model for studying therapeutic intervention in other neurodegenerative conditions such as Alzheimer's or Parkinson's disease where no preclinical diagnostic tests exist. In addition, HD manifests with a broad range of clinical symptoms and signs, many of them common to these other diseases, and involves widespread pathology throughout most of the brain. Understanding of HD pathogenesis is evolving, and I will present recent findings on identifying novel genetic modifiers of age of onset and disease progression, and their relevance for designing new therapeutics. In the main body of my lecture, I will give an overview of important approaches in development for targeting mutant HTT DNA and RNA, and in particular I will focus on the recent successful phase $1 \mathrm{~b} / 2 \mathrm{a}$ clinical trial testing the effects of RG6042 (formerly known as IONIS HTT Rx) in patients with early Huntington's Disease and present the results of the first successful HTT-lowering drug trial.
S. J. Tabrizi: F. Consultant/Advisory Board; Modest; F. Hoffmann-La Roche Ltd, Takeda Pharmaceuticals Ltd. F. Consultant/Advisory Board; Significant; Genentech.

\section{Concurrent Sessions}

\section{C01 From Carrier Screening to Infertility and Fetal Diagnostics}

\section{C01.1}

Consistency of carrier screening guidelines across seven populations and 408,00 individuals

\author{
A. Arjunan ${ }^{*}$, R. Ben-Shachar, R. Torres, K. Johansen \\ Taber, K. E. Kaseniit, D. Muzzey
}

Myriad Women's Health, South San Francisco, CA, United States

Introduction: Current American College of Obstetricians and Gynecologists (ACOG) guidelines recommend panethnic carrier screening for two conditions: cystic fibrosis and spinal muscular atrophy. In addition, ACOG recommends screening for approximately a dozen conditions in certain ethnicities. Here we analyzed carrier rates of 176 serious conditions to determine whether current guidelines support consistent care across all ethnicities.

Methods: Population-specific carrier rates of 176 Mendelian conditions were estimated using self-reported ethnicity in a cohort of over 408,000 individuals undergoing expanded carrier screening. Carrier screening guidelines were then analyzed to determine consistency across ethnicities.

Results: We analyzed 44 population-condition combinations, and found that the least frequent was cystic fibrosis (CF) in the Southeast Asian population (populationcondition carrier rate $0.6 \%, 1$ in 161). We then posited the question: how many disorders not recommended for screening are as frequent or more frequent than the least frequent disorder currently recommended by carrier-screening guidelines? Searching across other permutations revealed 191 additional population-condition carrier rates in excess of $0.6 \%$, suggesting that current guidelines are not consistently capturing reproductive risk across populations or conditions.

Conclusions: Many conditions have population-specific incidence comparable to $\mathrm{CF}$ in the Southeast Asian population - the rarest population-condition pair currently recommended for carrier screening by ACOG. Expanding guidelines to include pan-ethnic screening of additional conditions with comparable or higher incidence in select 
populations would improve equitability of medical care across all populations.

This study was funded by Myriad Genetics, Inc.

A. Arjunan: None. R. Ben-Shachar: A. Employment (full or part-time); Significant; Myriad Genetics, Inc. E. Ownership Interest (stock, stock options, patent or other intellectual property); Significant; Myriad Genetics, Inc. R. Torres: A. Employment (full or part-time); Significant; Myriad Genetics, Inc. E. Ownership Interest (stock, stock options, patent or other intellectual property); Significant; Myriad Genetics, Inc. K. Johansen Taber: A. Employment (full or part-time); Significant; Myriad Women's Health. E. Ownership Interest (stock, stock options, patent or other intellectual property); Significant; Myriad Genetics, Inc. K. E. Kaseniit: A. Employment (full or part-time); Significant; Myriad Women's Health. F. Consultant/Advisory Board; Significant; Myriad Women's Health. D. Muzzey: A. Employment (full or part-time); Significant; Myriad Women's Health. E. Ownership Interest (stock, stock options, patent or other intellectual property); Significant; Myriad Genetics, Inc.

\section{C01.2}

Expanded carrier screening of recessive disorders (CarrierTest) in clinical practice

J. Diblik ${ }^{*}$, M. Bittóová, F. Lhota, F. Zembol, L. Dohnalová, Z. Vilímová, I. Soldátová, M. Hrabíková, I. Pavlechová, M. Koudová, D. Stejskal

\section{GENNET s.r.o., Praha, Czech Republic}

Introduction: Screening for carrier status of recessive disorders is a standard part of preconception care. Expanded screening is based on simultaneous detection of variants in multiple genes that covers mutations prevalent in different human populations.

Methods: CarrierTest is a custom designed test based on amplicon sequencing of target regions in 77 genes for detection of 835 pathogenic mutations causing more than 61 inherited disorders.

Results: We have examined 10752 individuals from couples with infertility or recurrent pregnancy loss between April 2017 and October 2019. In total we have detected 3899 pathogenic variants, most often in following genes: GJB2 (in $4.9 \%$ of individuals), CFTR (3.6\%), SERPINA1 (2.6\%), DHCR7 (2.6\%), SMN1 (2.5\%), PAH (2.3\%), NBN (1.8\%), CYP21A2 (3.3\%), ATP7B (1.2\%), PMM2 $(1.1 \%), A C A D V L(1.1 \%)$ and ACADM $(1 \%)$. We have found a high risk of autosomal recessive disorder in 67 couples $(1.25 \%)$, most often both partners were carriers of mutations in GJB2 (18x), CFTR (13×), SMN1 (6×),

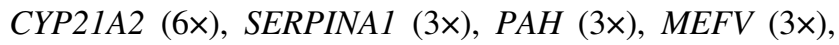

DHCR7 (2x), PMM2 (2x), ACADVL (2x), ACADM (2x), HEXA (2x) and ARSA (2x).

Conclusion: Results of CarrierTest provide the couples with information about the risk for their offspring. The severity depends on the gene and exact mutations. The findings thus must be assessed and communicated by a clinical geneticist so that couples can make informed decisions. The available preventive measures include preimplantation genetic testing, prenatal diagnosis as well as postnatal diagnosis for early treatment.

J. Diblík: None. M. Bittóová: None. F. Lhota: None. F. Zembol: None. L. Dohnalová: None. Z. Vilímová: None. I. Soldátová: None. M. Hrabíková: None. I. Pavlechová: None. M. Koudová: None. D. Stejskal: None.

\section{C01.3}

Exome sequencing reveals variants in known and novel candidate genes for severe sperm motility disorders

M. S. Oud ${ }^{1 *}$, B. J. Houston ${ }^{2}$, L. Volozonoka ${ }^{3,4}$, F. K. Mastrorosa $^{4}$, B. Alobaidi ${ }^{4}$, P. F. de Vries ${ }^{l}$, G. Astuti ${ }^{l}$,

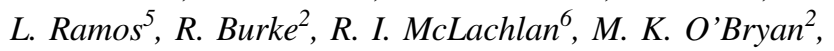
J. A. Veltman ${ }^{1,4}$, H. E. Chemes ${ }^{7}$, H. Sheth ${ }^{4,8}$

${ }^{1}$ Department of Human Genetics, Radboudumc, Nijmegen, Netherlands, ${ }^{2}$ Monash School of Biological Sciences, Clayton, Australia, ${ }^{3}$ Riga Stradins University, Riga, Latvia, ${ }^{4}$ Biosciences Institute Newcastle University, Newcastle, United Kingdom, ${ }^{5}$ Department of Gynaecology and Obstetrics, Radboudumc, Nijmegen, Netherlands, ${ }^{6} 6 \mathrm{Hudson}$ Institute of Medical Research, Clayton, Australia, ${ }^{7}$ Center for Research in Endocrinology, Buenos Aires Children's Hospital, Buenos Aires, Argentina, ${ }^{8}$ Foundation for Research in Genetics and Endocrinology, Ahmedabad, India

Severe sperm motility disorders are a form of male infertility characterized by immotile sperm, often in combination with a spectrum of structural abnormalities of the sperm flagellum. Depending on the sub-categorization, genetic factors explain up to $50 \%$ of these patients. We performed exome sequencing on 21 patients with severe sperm motility disorders to identify the underlying genetic defects. A diagnosis was reached in $9 / 21$ patients $(42.9 \%)$ due to variants in previously described genes CFAP43 (3 patients), CFAP44 (2 patients), QRICH2 (2 patients), DNAH1 (1 patient) and DNAH6 (1 patient). Furthermore, we identified seven patients with variants in novel spermatogenesis-related candidate genes: CFAP58, DNAH12, DRC1, MDC1, PACRG, SSPL2C and TPTE2. One other patient carried variants in four candidate genes. Electron microscopy imaging was available to further characterize the sperm flagellum phenotype in 4/9 diagnosed patients and 5/8 
patients with variants in candidate genes. This revealed short, irregular tails with thickening of the fibrous sheath (9/ 9 patients), abnormal outer dense fibers (5/9) and absence of dynein arms (2/9 patients), central microtubule pairs (6/9 patients) or mitochondrial sheath (9/9). RNA interference study in Drosophila melanogaster confirmed that loss of DNAH6, DNAH12 and QRICH2 expression resulted in male infertility, thus suggesting an evolutionary conserved role for these genes. Our results confirm an important role for five known genes and demonstrate that exome sequencing is an effective method to diagnose patients with severe motility disorders. Sequencing of additional patient cohorts is needed to further strengthen the role of the novel candidate genes in motility disorders.

M. S. Oud: None. B. J. Houston: None. L. Volozonoka: None. F. K. Mastrorosa: None. B. Alobaidi: None. P. F. de Vries: None. G. Astuti: None. L. Ramos: None. R. Burke: None. R. I. McLachlan: None. M. K. O’Bryan: None. J. A. Veltman: None. H. E. Chemes: None. H. Sheth: None.

\section{C01.4}

Identification of novel causes for male infertility: loss-offunction variants in $\mathrm{MSH} 4$ and $\mathrm{MSH} 5$

\section{J. Wyrwoll $^{1,2 *}$, C. Friedrich ${ }^{1}$, E. Kaminsky ${ }^{3}$, C. Krallmann ${ }^{2}$, S. Kliesch ${ }^{2}$, F. Tüttelmann ${ }^{1}$}

${ }^{1}$ Institute of Human Genetics, University of Münster, Münster, Germany, ${ }^{2}$ Centre of Reproductive Medicine and Andrology, Department of Clinical and Surgical Andrology, University Hospital, Münster, Germany, ${ }^{3}$ Praxis für Humangenetik Kaminsky, Hamburg, Germany

Infertility affects around $7 \%$ of all men and is in part due to azoospermia. Testicular sperm extraction (TESE) from biopsies in azoospermic patients offers the possibility to recover sperm that can be used for assisted reproduction. However, 2-10\% of azoospermic patients have meiotic arrest (MeiA). If MeiA is complete, TESE will not succeed. We performed whole exome sequencing (WES) in 68 patients with MeiA and negative TESE procedures. Alternative causes for infertility were excluded in advance. WES data was screened with focus on rare, biallelic variants [maximum allele frequency (MAF) $<1 \%$ in all databases], which are likely pathogenic (stop-, frameshift-, and splice site-mutations). We detected two novel genes, MSH4 and $\mathrm{MSH5}$, as possible causes for MeiA in men. Both genes are expressed predominantly in germ cells in humans and are associated with premature ovarian insufficiency in women. One of our MeiA patients carries biallelic loss of function variants in $\mathrm{MSH}$, while three azoospermic patients have homozygous variants in MSH5. MSH4 and MSH5 form a heterodimer, which binds DNA strands during prophase 1, stabilising the interaction between homologous chromosomes. Based on our data we propose that pathogenic variants in MSH4 and MSH5 cause autosomal recessive MeiA. If validated, screening of MSH4 and MSH5 in azoospermic patients before testicular biopsy may prevent unnecessary surgical procedures, if there is no chance of finding mature spermatozoa in the testis. This work was carried out within the frame of the DFG Clinical Research Unit 'Male Germ Cells: from Genes to Function'(CRU 326).

M. J. Wyrwoll: None. C. Friedrich: None. E. Kaminsky: None. C. Krallmann: None. S. Kliesch: None. F. Tüttelmann: None.

\section{C01.5}

Non-invasive prenatal diagnosis by genome-wide haplotyping of cell-free plasma DNA

H. Che $^{1 *}$, D. Villela ${ }^{1,2}$, E. Dimitriadou ${ }^{l}$, C. Melotte ${ }^{l}, N$. Brison $^{l}$, M. Neofytou ${ }^{l}, K$. Van Den Bogaert ${ }^{l}, O$. Tsuiko ${ }^{l}, K$. Devriendt $^{l}$, E. Legius ${ }^{I}$, M. Zamani Esteki ${ }^{1,3,4}$, T. Voet ${ }^{l}$, J. R. Vermeesch $^{l}$

${ }^{1}$ Center of Human Genetics, KU Leuven, Leuven, Belgium, ${ }^{2}$ Department of Genetics and Evolutionary Biology, Institute of Biosciences, University of Sao Paulo, Sao Paulo, Brazil, ${ }^{3}$ Department of Clinical Genetics, Maastricht University Medical Center, Maastricht, Netherlands, ${ }^{4}$ Department of Genetics and Cell Biology, GROW School for Oncology and Developmental Biology, Maastricht University, Maastricht, Netherlands

Introduction: While non-invasive prenatal testing for aneuploidies (NIPT-A) is widely implemented, there is an increasing need for universal approaches for noninvasive prenatal testing for monogenic diseases (NIPT-M). Here, we present a cost-effective, generic cell-free fetal DNA (cffDNA) haplotyping approach to scan the fetal genome for the presence of inherited monogenic diseases.

Methods: Families participating in the preimplantation genetic testing for monogenic disorders (PGT-M) program were recruited for this study. 250000 SNPs captured from maternal plasma DNA along with genomic DNAs from family members were massively parallel sequenced. Parental genotypes were phased via an available genotype from a close relative, and the fetal genome-wide haplotype and copy number were determined using cffDNA haplotyping analysis based on estimation and segmentation of fetal allele presence in the maternal plasma.

Results: In all families tested, mutational profiles from cffDNA haplotyping are consistent with embryo biopsy profiles. Genome-wide fetal haplotypes are on average $97 \%$ concordant with the newborn haplotypes and embryo haplotypes. 
Conclusion: We demonstrate that genome-wide targeted capture and sequencing of polymorphic SNPs from maternal plasma cell free DNA (cfDNA) allows haplotyping and copy number profiling of the fetal genome during pregnancy. The method enables the accurate reconstruction of the fetal haplotypes and can be easily implemented in clinical practice.

Grants: This work was funded by KULeuven funding (C1/018), the Horizon 2020 WIDENLIFE: 692065 to J. R. V. and T.V and by the Fonds voor Wetenschappelijk Onderzoek (FWO-Vlaanderen: G080217N) to J. V. D. V. was supported by FAPESP (2017/23448-8).

H. Che: None. D. Villela: None. E. Dimitriadou: None. C. Melotte: None. N. Brison: None. M. Neofytou: None. K. Van Den Bogaert: None. O. Tsuiko: None. K. Devriendt: None. E. Legius: None. M. Zamani Esteki: None. T. Voet: None. J. R. Vermeesch: B. Research Grant (principal investigator, collaborator or consultant and pending grants as well as grants already received); Modest; Agilent.

\section{C01.6}

Trio exome/whole exome sequencing in prenatal diagnosis: Experiences from $>400$ cases shows a high diagnostic yield and a great benefit for pregnancy management and genetic counselling.

\author{
H. Gabriel ${ }^{*}$, C. Wilhelm ${ }^{2}$, F. Battke ${ }^{1}$, E. Goldmann ${ }^{2}$, \\ C. Bus ${ }^{2}$, S. Biskup ${ }^{1}$ \\ ${ }^{1}$ CeGaT, Tuebingen, Germany, ${ }^{2}$ Praxis für Humangenetik, \\ Tuebingen, Germany
}

Whole exome sequencing (WES) and trio exome sequencing is becoming the first-tier diagnostic test for many unclear genetic conditions. However, in prenatal testing, several challenges have to be addressed: turn-around-time, patient acceptability, additional findings, interpretation of unclear variants, and ethical dilemmas. A cohort of $>400$ unselected fetuses with varying structural anomalies seen during routine ultrasonography were analysed after amniocentesis or chorionic villus sampling. In 326 samples, trio exome sequencing was performed. Only likely pathogenic and pathogenic (according to the current ACMG guideline; Richards et al., 2015), in genes to be potential involved in the observed fetal phenotype were considered. $30 \%$ of the cases were clearly solved by trio exome sequencing, meaning that class 4 or 5 variants consistent with the fetal phenotype were found. An additional $12 \%$ cases with variants very likely causative for the phenotype were detected. In most of these cases only limited clinical information was available or only soft markers (e.g. increased nuchal translucency) were present. The mean turn-around-time (TAT) was 18 days with a shortest TAT of 5 days. In this study, we have shown the value of trio exome sequencing for the prenatal genetic diagnosis of fetuses with varying structural anomalies. A fast, stable, and reliable process including sample processing, data analysis, and comprehensive reporting is required to provide patients with the maximal benefit. In summary, prenatal exome sequencing offers a feasible diagnostic approach for fetuses with anomalies, but a multidisciplinary team to cover all ethical, psychological, social and medical aspects is required.

H. Gabriel: None. C. Wilhelm: None. F. Battke: None. E. Goldmann: None. C. Bus: None. S. Biskup: None.

\section{C02 Intellectual Disability}

\section{C02.1}

SCAF4 loss of function in humans and Drosophila implicates mRNA transcriptional termination in neurodevelopmental disorders

A. Fliedner $^{1 *}$, P. Kirchner ${ }^{1}$, K. E. Agre ${ }^{2}$, I. de Graaf-van de Laar $^{3}$, M. Dutra Clarke ${ }^{4}$, L. Davis-Keppen ${ }^{5}$, A. B. Ekici ${ }^{1}$, A.

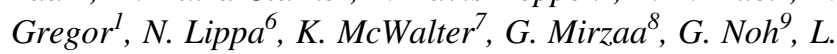

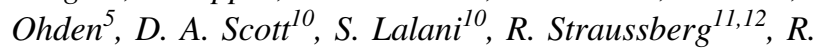
Cohen $^{13,12}$, A. Wiesener ${ }^{1}$, C. Zweier ${ }^{1}$

${ }^{1}$ Institute of Human Genetics, Friedrich-Alexander-Universität Erlangen-Nürnberg, Erlangen, Germany, ${ }^{2}$ Clinical Genomics, Mayo Clinic, Rochester, MN, United States, ${ }^{3}$ Department of Clinical Genetics, Erasmus MC, University Medical Center Rotterdam, Rotterdam, Netherlands, ${ }^{4}$ Division of Genetics and Genomic Medicine, Department of Pediatrics, University of California-Irvine, School of Medicine, Irvine, CA, United States, ${ }^{5}$ University of South Dakota, Sanford School of Medicine, Sioux Falls, SD, United States, ${ }^{6}$ Institute for Genomic Medicine, Columbia University Medical Center, New York, NY, United States, ${ }^{7}$ Clinical Genomics, GeneDx, Gaithersburg, MD, United States, ${ }^{8}$ Department of Pediatrics, University of Washington, Seattle, WA, United States, ${ }^{9}$ Department of Genetics, Southern California Permanente Medical Group, Fontana, CA, United States, ${ }^{10}$ Department of Molecular and Human Genetics, Baylor College of Medicine, Houston, TX, United States, ${ }^{11}$ INeurogenetics Clinic, Neurology Unit, Schneider Children Medical Center, Petah Tikvah, Israel, ${ }^{12}$ Sackler School of Medicine Tel Aviv University, Ramat Aviv, Israel,

${ }^{13}$ Neurology Institute and Epilepsy Center, Schneider Children Medical Center, Petah Tikvah, Israel

Regulation of gene expression is highly complex and involves a large number of different processes including mRNA termination at polyA sites. This is mediated by RNA polymerase II and requires binding of SR-related and CTD-associated factor 4 (SCAF4). SCAF4 represses the 
usage of early polyA sites and thus prevents usage of alternative last exons (ALEs) and formation of truncated, non-functional proteins. SCAF4 is expressed in all adult and fetal tissues, with high expression in brain, and has not been associated with disease, yet. By exome sequencing and international matchmaking we assembled six truncating, two splice-site and one missense variant in SCAF4, all but one de novo. All individuals presented with a variable neurodevelopmental disorder with developmental delay, mild to severe intellectual disability and various other aspects such as epilepsy (6/9), behavioral anomalies (6/9) and skeletal (3/9) or renal (3/9) anomalies. Knockdown of the SCAF4 orthologue CG4266 in the model organism Drosophila melanogaster resulted in impaired locomotor function in the climbing assay and impaired learning and short-term memory tested by the courtship conditioning paradigm. Furthermore, we observed an increased number of active zones in larval neuromuscular junctions, representing large glutamatergic synapses. These observations indicate a role of CG4266 in nervous system development and function and support a role of its human orthologue SCAF4 in neurodevelopmental disorders. We establish likely gene-disruptive variants in SCAF4 as causative for a variable neurodevelopmental disorder and thus implicate disturbed mRNA termination in impairment of neurodevelopment and cognition.

A. Fliedner: None. P. Kirchner: None. K. E. Agre: None. I. de Graaf-van de Laar: None. M. Dutra Clarke: None. L. Davis-Keppen: None. A. B. Ekici: None. A. Gregor: None. N. Lippa: None. K. McWalter: A. Employment (full or parttime); Significant; GeneDx. G. Mirzaa: None. G. Noh: None. L. Ohden: None. D. A. Scott: None. S. Lalani: None. R. Straussberg: None. R. Cohen: None. A. Wiesener: None. C. Zweier: None.

\section{C02.2}

Mutation-specific pathophysiological mechanisms in a new SATB1-associated neurodevelopmental disorder

J. den Hoed ${ }^{1 *}$, E. de Boer ${ }^{2,3}$, N. Voisin ${ }^{4}$, N. Guex ${ }^{4}$, L. Snijders Blok ${ }^{1,2,3}$, J. Chrast ${ }^{4}$, The DDD study, L. Manwaring ${ }^{5}$, M. Willing 5 , A. Waheeb ${ }^{6,7}$, M. Osmond ${ }^{7}, K$. McWalter ${ }^{8}$, A. Vitobello ${ }^{9,10}$, F. Demurger ${ }^{11}$, A. Lavillaureix $^{12}$, S. Odent ${ }^{12}$, B. Mazel ${ }^{10}$, L. Faivre ${ }^{10}$, I. Thiffault $^{13,14}$, C. Schwager ${ }^{13,14}$, S. M. Amudhavalli ${ }^{13,14}$, J. A. Rosenfeld $^{15}$, K. Radtke ${ }^{16}$, E. Preiksaitiene ${ }^{17,18}$, E. Ranza $^{19,20}$, C. Depienne ${ }^{21}$, A. Kuechler ${ }^{21}$, S. Mohammed ${ }^{22}$, Y. Hamzavi Abedi ${ }^{23,24}$, V. R. Bonagura ${ }^{25,24}$, B. Zuccarelli ${ }^{26}$, B. Horist ${ }^{27}$, V. Krishnamurthy ${ }^{27}$, A. A. Kattentidt-

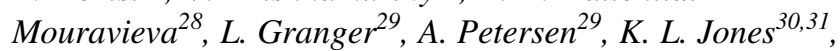
M. Sinnema ${ }^{32}$, A. P. A. Stegmann ${ }^{2,32}$, R. Newbury-Ecob ${ }^{33}$, U. Kini ${ }^{34}$, D. F. Newbury ${ }^{35}$, C. Gilissen ${ }^{2,36}$, H. Brunner ${ }^{2,3,32}$,
T. Kleefstra ${ }^{2,3}$, A. Reymond ${ }^{4}$, L. E. L. M. Vissers ${ }^{2,3}$, S. E. Fisher $^{1,3}$

${ }^{I}$ Max Planck Institute for Psycholinguistics, Nijmegen, Netherlands, ${ }^{2}$ Radboudumc, Nijmegen, Netherlands, ${ }^{3}$ Donders Institute for Brain, Cognition and Behaviour, Nijmegen, Netherlands, ${ }^{4}$ University of Lausanne, Lausanne, Switzerland, ${ }^{5}$ Washington University School of Medicine, St. Louis, MO, United States, ${ }^{6}$ Children's Hospital of Eastern Ontario, Ottawa, ON, Canada, ${ }^{7}$ Children's Hospital of Eastern Ontario Research Institute, Ottawa, ON, Canada, ${ }^{8}$ GeneDx, Gaithersburg, MD, United States, ${ }^{9}$ Université de Bourgogne Franche-Comté, Dijon, France, ${ }^{10}$ Centre Hospitalier Universitaire de Dijon, Dijon, France, ${ }^{11}$ Vannes hospital, Vannes, France, ${ }^{12}$ Rennes University, Institute of Genetics and Development of Rennes (IGDR), Rennes, France, ${ }^{13}$ University of Missouri-Kansas City School of Medicine, Kansas City, MO, United States, ${ }^{14}$ Children's Mercy Hospital, Kansas City, MO, United States, ${ }^{15}$ Baylor College of Medicine, Houston, TX, United States, ${ }^{16}$ Ambry Genetics, Aliso Viejo, CA, United States, ${ }^{17}$ Vilnius University, Vilnius, Lithuania, ${ }^{18}$ Vilnius University Hospital Santaros Klinikos, Vilnius, Lithuania, ${ }^{19}$ University of Geneva, Geneva, Switzerland, ${ }^{20}$ University Hospitals of Geneva, Geneva, Switzerland, ${ }^{21}$ University Hospital Essen, University of Duisburg-Essen, Essen, Germany, ${ }^{22}$ Guy's Hospital, London, United Kingdom, ${ }^{23}$ Northwell Health, Great Neck, NY, United States, ${ }^{24}$ Donald and Barbara Zucker School of Medicine at Hofstra/Northwell, Hempstead, NY, United States, ${ }^{25}$ Feinstein Institutes for Medical Research, Manhasset, NY, United States, ${ }^{26}$ The University of Kansas School of Medicine Salina Campus, Salina, KS, United States, ${ }^{27}$ Pediatrics \& Genetics Alpharetta, Alpharetta, GA, United States, ${ }^{28}$ Zuidwester, Middelharnis, Netherlands, ${ }^{29}$ Randall Children's Hospital at Legacy Emanuel, Portland, OR, United States, ${ }^{30}$ Children's Hospital of the King's Daughters, Norfolk, VA, United States, ${ }^{31}$ Eastern Virginia Medical School, Norfolk, VA, United States, ${ }^{32}$ Maastricht University Medical Center+, Maastricht, Netherlands, ${ }^{33}$ St Michael's Hospital Bristol, University Hospitals Bristol NHS Foundation Trust, Bristol, United Kingdom, ${ }^{34}$ Oxford University Hospitals NHS Foundation Trust, Oxford, United Kingdom, ${ }^{35}$ Oxford Brookes University, Oxford, United Kingdom, ${ }^{36}$ Radboud Institute for Molecular Life Sciences, Nijmegen, Netherlands

Introduction: Special AT-rich sequence-binding protein-1 (SATB1) is a transcription factor that has not been implicated in neurodevelopmental disorders, but was recently identified as a dominant candidate gene in two large-scale meta-analyses, together comprising $>65,000$ patients with 
neurodevelopmental disorders, including intellectual disability (ID) and/or autism spectrum disorders. A homozygous knockout of the orthologous Satbl leads to mice lethality, demonstrating its importance in early development.

Methods and results: Here, we report on 26 individuals with SATB1 variants. Seventeen patients have missense variants predicted to be deleterious, and nine have truncating variants, three of which predicted to escape nonsense-mediated decay. 21 of 26 variants $(80 \%)$ were confirmed to be de novo in origin. Patients showed a broad phenotypic spectrum, including ID and/or neurodevelopmental delay, epilepsy, dental abnormalities and aspecific brain MRI findings. Additionally, patients with missense variants are more severely affected than those with truncating variants. We characterized the effects of eight different missense mutations, including two recurrent variants, in cell-based assays. Compatible with a gain-offunction effect of missense variants, mutated SATB1 proteins showed altered nuclear localization and increased transcriptional repression on two characterized targets (IgHMAR site and the IL2 promoter). Consistent with this hypothesis, fluorescent recovery after photobleaching (FRAP) experiments demonstrated a decrease in mobility of mutant SATB1, suggesting stabilization of DNA binding underlying the increased transcriptional activity. Transcriptome analysis is currently ongoing to further substantiate these findings.

Conclusions: In summary, through multi-center collaborations, we show that SATB1 is associated with an autosomal dominant neurodevelopmental disorder, resulting from distinct mutation-specific pathophysiological mechanisms.

J. den Hoed: None. E. de Boer: None. N. Voisin: None. N. Guex: None. L. Snijders Blok: None. J. Chrast: None. L. Manwaring: None. M. Willing: None. A. Waheeb: None. M. Osmond: None. K. McWalter: None. A. Vitobello: None. F. Demurger: None. A. Lavillaureix: None. S. Odent: None. B. Mazel: None. L. Faivre: None. I. Thiffault: None. C. Schwager: None. S. M. Amudhavalli: None. J. A. Rosenfeld: None. K. Radtke: None. E. Preiksaitiene: None. E. Ranza: None. C. Depienne: None. A. Kuechler: None. S. Mohammed: None. Y. Hamzavi Abedi: None. V. R. Bonagura: None. B. Zuccarelli: None. B. Horist: None. V. Krishnamurthy: None. A. A. Kattentidt-Mouravieva: None. L. Granger: None. A. Petersen: None. K. L. Jones: None. M. Sinnema: None. A. P. A. Stegmann: None. R. NewburyEcob: None. U. Kini: None. D. F. Newbury: None. C. Gilissen: None. H. Brunner: None. T. Kleefstra: None. A. Reymond: None. L. E. L. M. Vissers: None. S. E. Fisher: None.

\section{C02.3}

De novo frameshift variants in the neuronal splicing factor NOVA2 result in a common C-terminal part and cause a severe form of neurodevelopmental disorder

\section{F. Mattioli ${ }^{1 *}$, G. Hayot ${ }^{l}$, N. Drouot ${ }^{l}$, B. Isidor ${ }^{2}, J$.} Courraud $^{l}$, M. Hinckelmann ${ }^{1}$, F. Tran Mau-Them ${ }^{3,4}$, C. Sellier ${ }^{1}$, A. Goldman ${ }^{5}$, A. Telegrafi ${ }^{6}$, A. Boughton ${ }^{7}$, C.

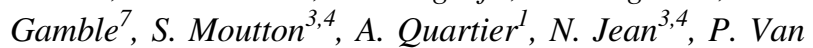

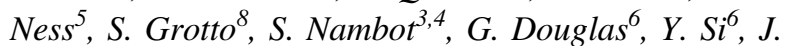

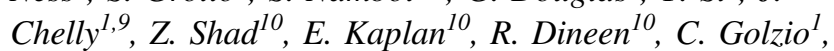
N. Charlet ${ }^{1}$, J. Mandel ${ }^{1}$, A. Piton ${ }^{1,9}$

${ }^{1}$ IGBMC, Illkirch, France, ${ }^{2} \mathrm{CHU}$ de Nantes, Nantes, France, ${ }^{3} \mathrm{CHU}$ de Dijon, Dijon, France, ${ }^{4}$ Burgundy University, Dijon, France, ${ }^{5}$ Baylor College of Medicine, Houston, TX, United States, ${ }^{6}$ GeneDx, Gaithersburg, MD, United States, ${ }^{7}$ Cook Children's Genetics, Fort Worth, TX, United States, ${ }^{8}$ AP-HP Robert-Debré, Paris, France, ${ }^{9}$ Hôpitaux Universitaires de Strasbourg, Strasbourg, France, ${ }^{10}$ University of Illinois College of Medicine, Chicago, IL, United States

Monogenic forms of intellectual disability (ID) are characterized by an extreme heterogeneity, with more than 700 genes now implicated. The most frequent cause of monogenic ID - the fragile X-syndrome- is due to the absence of the RNA-binding protein (RBP) FMRP. Many other ID genes have been implicated in the RNA metabolism. NOVA2 is a RBP known to regulate neuron specific alternative splicing (AS). We identified 6 individuals with de novo frameshift variants in NOVA2 affected with a neurodevelopmental disorder characterized by ID, motor and speech delay, autistic features, spasticity or ataxic gait and abnormal brain MRI. All variants cluster in a small GCrepeat rich region, located in the last exon, indicating that the mutated transcript might escape to nonsense mediated decay. All 6 frameshift variants lead to the addition of a novel common C-terminal part instead of the last KH RNAbinding domain, suggesting a dominant negative mechanism. We detected 41 genes differentially spliced after downregulation of NOVA2 in human neuronal cells. The NOVA2 variant protein shows decreased ability to bind target RNA sequences and to regulate target AS events. It also fails to complement the effect on neurite outgrowth induced by NOVA2 downregulation in vitro and to rescue alterations of retinotectal axonal pathfinding induced by loss of NOVA2 ortholog in zebrafish. Our results showed that truncating mutations in NOVA2 cause a syndromic form of ID, suggesting a hypomorphic mechanism rather than a full heterozygous loss of function, although a specific contribution of the novel C-terminal extension cannot be excluded. 
F. Mattioli: None. G. Hayot: None. N. Drouot: None. B. Isidor: None. J. Courraud: None. M. Hinckelmann: None. F. Tran Mau-Them: None. C. Sellier: None. A. Goldman: None. A. Telegrafi: A. Employment (full or part-time); Significant; Genedx. A. Boughton: None. C. Gamble: None. S. Moutton: None. A. Quartier: None. N. Jean: None. P. Van Ness: None. S. Grotto: None. S. Nambot: None. G. Douglas: A. Employment (full or part-time); Significant; GeneDx. Y. Si: A. Employment (full or part-time); Significant; GeneDx. J. Chelly: None. Z. Shad: None. E. Kaplan: None. R. Dineen: None. C. Golzio: None. N. Charlet: None. J. Mandel: None. A. Piton: None.

\section{C02.4}

Location, location, location: protein truncating variants in different loci of $S R C A P$ cause three distinct neurodevelopmental disorders, associated with distinctive DNA methylation signatures

D. Rots $^{1 *}$, E. Chater-Diehl ${ }^{2}$, A. J. M. Dingemans ${ }^{1}$, M. Siu ${ }^{2}$, C. Cytrynbaum ${ }^{2}$, N. Hoang ${ }^{2}$, S. Walker ${ }^{2}$, S. Scherer ${ }^{2}, R$. Pfundt $^{l}$, T. Rinne ${ }^{l}$, T. Gardeitchik ${ }^{l}$, B. B. A. de Vries ${ }^{l}$, C. T. R. M. Stumpel ${ }^{3}$, S. J. C. Stevens ${ }^{4}$, J. van Harssel ${ }^{5}$, D. G. M. Bosch $^{5}$, K. L. I. van Gassen ${ }^{5}$, E. van Binsbergen ${ }^{5}$, C. M. de

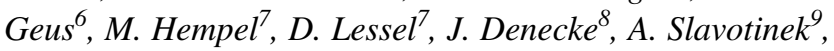
J. Strober ${ }^{9}$, L. B. Ousager ${ }^{10}$, M. Larsen ${ }^{10}$, L. SchultzRogers $^{11}$, E. Morava ${ }^{11}$, E. W. Klee ${ }^{11}$, I. R. Berry ${ }^{12}$, J. Campbell $^{13}$, K. Lindstrom ${ }^{14}$, A. M. Neumeyer ${ }^{15}$, J. A. Radley $^{16}$, C. Phornphutkul ${ }^{17}$, W. G. Wilson ${ }^{18}$, B. Schmidt ${ }^{18}$, S. Meyn ${ }^{2}$, K. Ounap ${ }^{19}$, K. Reinson ${ }^{19}$, S. Pajusalu ${ }^{19,20}$, C. Ruivenkamp $^{21}$, A. van Haeringen ${ }^{21}$, R. Cuperus $^{22}$, GeneDx inc., L. E. L. M. Vissers ${ }^{l}$, H. G. Brunner ${ }^{1,4}$, T. Kleefstra ${ }^{l}$, D. A. Koolen ${ }^{1}$, R. Weksberg ${ }^{2}$

${ }^{1}$ Radboudumc, Nijmegen, Netherlands, ${ }^{2}$ The Hospital for Sick Children, Toronto, ON, Canada, ${ }^{3}$ Department of Clinical Genetics and GROW-School for Oncology and Developmental Biology, Maastricht University Medical Center, Maastricht, Netherlands, ${ }^{4}$ Maastricht University Medical Center, Maastricht, Netherlands, ${ }^{5}$ University Medical Center Utrecht, Utrecht, Netherlands, ${ }^{6}$ University of Groningen, University Medical Center Groningen, Groningen, Netherlands, ${ }^{7}$ Institute of Human Genetics, University Medical Center Hamburg-Eppendorf, Hamburg, Germany, ${ }^{8}$ Department of Pediatrics, University Medical Center Hamburg-Eppendorf, Hamburg, Germany, ${ }^{9}$ Department of Pediatrics, UCSF, San Francisco, CA, United States, ${ }^{10}$ Odense University Hospital, Odense, Denmark, ${ }^{11}$ Mayo Clinic, Rochester, NY, United States, ${ }^{12}$ St. James's University Hospital, Leeds, United Kingdom, ${ }^{13}$ Chapel Allerton Hospital, Leeds, United Kingdom, ${ }^{14}$ Phoenix Children's Hospital, Phoenix, AZ, United States, ${ }^{15}$ Massachusetts General Hospital for Children, Harvard Medical
School, Boston, MA, United States, ${ }^{16}$ Birmingham Women's and Children's Hospitals NHS Foundation Trust, Birmingham, United Kingdom, ${ }^{17}$ Warren Alpert Medical School of Brown University, Providence, RI, United States, ${ }^{18}$ University of Virginia School of Medicine, Charlottesville, VA, United States, ${ }^{19}$ Tartu University Hospital and Institute of Clinical Medicine, Tartu, Estonia, ${ }^{20}$ Yale University School of Medicine, New Haven, CT, United States, ${ }^{21}$ Leiden University Medical Center, Leiden, Netherlands, ${ }^{22}$ Department of Paediatrics, Juliana Children's Hospital HAGA, the Hague, Netherlands

SRCAP functions in a chromatin remodeling complex, involved in epigenetic regulation of gene expression. Protein-truncating variants (PTVs) in the last two exons of SRCAP cause Floating-Harbor syndrome (FLHS), which is characterized by typical facial features, short stature with delayed bone age and expressive speech delay. We report on novel genotype-phenotype correlations, associated with SRCAP PTVs outside the FLHS locus.

We identified 21 probands with PTVs upstream, and 5 with PTVs downstream of the reported FLHS locus. Most patients presented with speech delay and intellectual disability. In contrast to FLHS, they have normal height and bone age. Moreover, facial-recognition algorithms on 2D-photos showed that patients with upstream and downstream PTVs do not cluster with the FLHS-facial gestalt. The upstream PTVs result in subtle facial dysmorphism, autism spectrum disorder, severe behavioral problems and joint issues, such as hypermobility and premature degeneration. In contrast, patients with downstream PTVs have neurodevelopmental delay and Marfanoid habitus.

At the molecular level, FLHS is characterized by a unique DNA methylation (DNAm) signature consisting of $\sim 570$ differentially methylated CpGs. The DNAm profiles of the upstream and downstream PTVs yielded different signatures from FLHS.

In summary, we delineate the phenotype of patients with SRCAP PTVs outside the FLHS locus. None of the patients show clinical features typical for FLHS. Analysis at the molecular level suggests that each clinical entity is associated with its own molecular mechanism. We propose that the upstream PTVs result in haploinsufficiency, whereas the downstream PTVs and FLHS-causing variants result in distinct dominant-negative or gain-of-function effect.

D. Rots: None. E. Chater-Diehl: None. A. J. M. Dingemans: None. M. Siu: None. C. Cytrynbaum: None. N. Hoang: None. S. Walker: None. S. Scherer: None. R. Pfundt: None. T. Rinne: None. T. Gardeitchik: None. B. B. A. de Vries: None. C. T. R. M. Stumpel: None. S. J. C. Stevens: None. J. van Harssel: None. D. G. M. Bosch: None. K. L. I. van Gassen: None. E. van Binsbergen: None. C. M. de Geus: 
None. M. Hempel: None. D. Lessel: None. J. Denecke: None. A. Slavotinek: None. J. Strober: None. L. B. Ousager: None. M. Larsen: None. L. Schultz-Rogers: None. E. Morava: None. E. W. Klee: None. I. R. Berry: None. J. Campbell: None. K. Lindstrom: None. A. M. Neumeyer: None. J. A. Radley: None. C. Phornphutkul: None. W. G. Wilson: None. B. Schmidt: None. S. Meyn: None. K. Ounap: None. K. Reinson: None. S. Pajusalu: None. C. Ruivenkamp: None. A. van Haeringen: None. R. Cuperus: None. L. E. L. M. Vissers: None. H. G. Brunner: None. T. Kleefstra: None. D. A. Koolen: None. R. Weksberg: None.

\section{C02.5}

Mechanistic dissection of chromatin topology disruption in the 5q14.3 MEF2C locus as an indirect driver of neurodevelopmental disorders

K. Mohajeri ${ }^{1,2}$, E. D'haene $^{3 *}$, R. Yadav ${ }^{1,2}, H . G u^{4,5}, B$. Menten $^{3}$, A. Presser Aiden ${ }^{4,5}$, C. Lowther ${ }^{1,2}$, S. Erdin ${ }^{1,2}$, E. Lieberman Aiden ${ }^{4}$, J. Gusellal, ${ }^{1,2}$ S. Vergult ${ }^{3}, M$.

Talkowski ${ }^{1,2}$

${ }^{1}$ Center for Genomic Medicine, Massachusetts General Hospital, Boston, MA, United States, ${ }^{2}$ Program in Medical and Population Genetics, Broad Institute, Cambridge, MA, United States, ${ }^{3}$ Center for Medical Genetics, Dept. of Biomolecular Medicine, Ghent University, Gent, Belgium, ${ }^{4}$ The Center for Genome Architecture, Baylor College of Medicine, Houston, TX, United States, ${ }^{5}$ Department of Molecular and Human Genetics, Baylor College of Medicine, Houston, TX, United States

Structural variants have the potential to create long-range positional effects, uncouple genes from regulatory elements, and facilitate aberrant 3D chromatin folding. In an independent study, we revealed a significant enrichment of intergenic balanced chromosomal abnormality (BCA) breakpoints from congenital anomaly cases at chromosome 5q14. 3. All $115 q 14.3$ BCA carriers had breakpoints disrupting the topologically associated domain (TAD) housing $M E F 2 C$, a known driver of neurodevelopmental disorders. In a second study, we showed, using $4 \mathrm{C}$-seq, that $M E F 2 C$ forms proximal and distal interaction loops within this $\mathrm{TAD}$, contacting multiple neuronal enhancers.

To understand the functional impact of these BCAs, we performed a mechanistic dissection of the $3 \mathrm{D}$ regulatory network at the 5q14.3 locus and its constituent functional elements using Cas9-based genome editing. We generated $>200$ cell lines, representing deletions of $M E F 2 C$ alongside five $M E F 2 C$ TAD boundary and interaction loop targets in iPS-derived neural stem cells (NSCs) and cortical induced neurons (iNs). We profiled changes in expression and 3D chromatin interactions within these models using RNA-seq and $4 \mathrm{C}$-seq. $M E F 2 C$ was variably differentially expressed upon deletion of proximal and distal $M E F 2 C$ loop regions, with effects depending on cell type and variant class. Underlying chromatin interaction patterns revealed evidence of loop maintenance in these models, possibly via CTCF buffering, highlighting compensatory mechanisms against 3D chromatin disruption. In contrast, deletion of the proximal TAD boundary facilitated increased contacts with predicted enhancers in the adjacent TAD.

Our results suggest novel regulatory mechanisms driving phenotypic outcomes for the 5q14.3 region, with significant implications for interpretation of pathogenic structural variation.

K. Mohajeri: None. E. D'haene: None. R. Yadav: None. H. Gu: None. B. Menten: None. A. Presser Aiden: None. C. Lowther: None. S. Erdin: None. E. Lieberman Aiden: None. J. Gusella: None. S. Vergult: None. M. Talkowski: None.

\section{C02.6}

Crispr-Cas9-engineered Pigv ${ }^{341 E}$ mouse model mirrors the human phenotype, reveals a hippocampal synaptic defect and suggests a major role of impaired Abl1/ Ephrin signaling pathway in GPI anchor deficiency

M. Rodríguez de los Santos ${ }^{1,2,3^{*}}$, M. Rivalan ${ }^{4}, F . S$. David $^{3,5}$, A. Knaus ${ }^{3}$, A. Stumpf $f^{6}$, L. Moreno Velasquez ${ }^{6}$, A. Voigt $^{6}$, D. Mattei ${ }^{7}$, M. Long ${ }^{4}$, L. Wittler ${ }^{8}$, B. Timmermann ${ }^{9}$, D. Horn ${ }^{1}$, S. Mundlos ${ }^{1,2,10}$, U. Kornak ${ }^{1,10}$, D. Schmitz ${ }^{6}$, Y. Winter $^{4}$, P. M. Krawitz ${ }^{3}$

${ }^{1}$ Institute for Medical Genetics and Human Genetics, Charité - Universitätsmedizin Berlin, Berlin, Germany, ${ }^{2}$ Max-Planck-Institute for Molecular Genetics, FG Development and Disease, Berlin, Germany, ${ }^{3}$ Institute for Genomic Statistics and Bioinformatics, University of Bonn, Bonn, Germany, ${ }^{4}$ Animal Outcome Core Facility of the NeuroCure Center, Charité - Universitätsmedizin Berlin and Institute of Cognitive Neurobiology, Humboldt University Berlin, Berlin, Germany, ${ }^{5}$ Institute of Human Genetics, University of Bonn, Faculty of Medicine \& University Hospital Bonn, Bonn, Germany, ${ }^{6}$ Neuroscience Research Center, Charité - Universitätsmedizin Berlin, Berlin, Germany, ${ }^{7}$ Institute of Veterinary Pharmacology and Toxicology, University of Zurich, UZH-Zürich, Zürich, Switzerland, ${ }^{8}$ Max-Planck-Institute for Molecular Genetics, Department Developmental Genetics, Berlin, Germany, ${ }^{9}$ Max Planck Institute for Molecular Genetics, Sequencing Core Facility, Berlin, Germany, ${ }^{10}$ Berlin-Brandenburg Center for Regenerative Therapies, Charité - Universitätsmedizin Berlin, Berlin, Germany

Pathogenic germline mutations in PIGV result in a glycosylphosphatidylinositol biosynthesis deficiency (GPIBD). 
Patients with biallelic mutations in genes of this molecular pathway show cognitive impairments, a psychomotor delay and often suffer from epilepsies. Thus far, the pathophysiology underlying the disease remains unclear and suitable rodent models were lacking. Hence, we generated a mouse model using CRISPR-Cas9 to introduce the most prevalent hypomorphic mutation observed in European patients, that is also conserved in mice Pigv:c.1022 C>A (p.Ala341Glu). At the behavioral level, $P i g v^{341 \mathrm{E}}$ mice mutants showed deficits in motor coordination and cognitive impairments in long-term spatial memory. Furthermore, Pigv ${ }^{341 \mathrm{E}}$ mice displayed alterations in social behavior and sleep patterns reflecting the human phenotype. Immunohistochemistry showed a decreased synaptophysin immunoreactivity in hippocampal CA1 and electrophysiology recordings demonstrated a reduced hippocampal synaptic transmission in $P i g v^{341 E}$ mice that may explain impaired memory formation. In addition, we performed single cell RNA sequencing of hippocampal cells of adult Pigv $v^{341 \mathrm{E}}$ mice that exhibit changes of gene expression, most prominently in a microglia subtype, subicular neurons and CA3 pyramidal neurons. A significant reduction of Abll transcripts in several cell clusters suggests a link to the signaling pathway of GPI-anchored ephrins, which are known to play a major role in synapse formation and plasticity. In summary, we present here the first mouse model with a patient-specific hypomorphic mutation that mirrors the human phenotype and will allow us to explore the pathophysiology in GPIBDs in future studies.

M. Rodríguez de los Santos: None. M. Rivalan: None. F. S. David: None. A. Knaus: None. A. Stumpf: None. L. Moreno Velasquez: None. A. Voigt: None. D. Mattei: None. M. Long: None. L. Wittler: None. B. Timmermann: None. D. Horn: None. S. Mundlos: None. U. Kornak: None. D. Schmitz: None. Y. Winter: None. P. M. Krawitz: None.

\section{C03 Novel Bioinformatic and Machine-Learning Methods}

\section{C03.1}

\section{Reconciling GWAS and Mendelian genetics through} core-gene identification

O. B. Bakker ${ }^{I *}$, A. Claringbould ${ }^{l}$, H. Westra ${ }^{l}$, S. Mulcahy

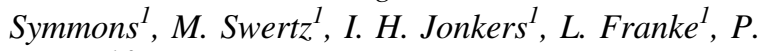
Deelen $^{1,2}$

${ }^{1}$ University Medical Center Groningen, Groningen, Netherlands, ${ }^{2}$ University Medical Center Utrecht, Utrecht, Netherlands

Genome-wide association studies (GWAS) have identified thousands of disease susceptibility loci, however the omnigenic model suggests that many of the genes in these loci might not directly be involved in core disease pathways. The omnigenic model postulates that most genes in the GWAS loci are peripheral genes that exert smaller downstream effects on genes that directly affect the phenotype (core genes). These core genes are expected to overlap with genes that result in rare Mendelian diseases when mutated.

In order to map the relationships between peripheral and core genes, we re-analysed 31,499 publicly available RNAseq samples from various tissues and cell-types to calculate a gene-gene co-regulation network. We then integrated the co-regulation network with GWAS summary statistics to identify genes that are co-regulated with a large number of GWAS genes. We hypothesise that these genes are more likely to be core disease genes.

As a proof of concept, we applied this gene prioritization strategy to a height GWAS and show that prioritized core genes are strongly enriched for Mendelian disease genes for both disproportionate short and tall stature (AUC 0.69 and 0.86 respectively) as expected under the omnigenic model.

The enrichment of Mendelian disease genes among the predicted core genes suggest that complex disease and rare disease genetics are on two ends of the same spectrum. We therefore expect that our method can ultimately be used to better understand common diseases and we hope to predict new rare disease genes.

O. B. Bakker: None. A. Claringbould: None. H. Westra: None. S. Mulcahy Symmons: None. M. Swertz: None. I. H. Jonkers: None. L. Franke: None. P. Deelen: None.

\section{C03.2}

\section{Improved de novo mutation detection through deep} learning

K. Šablauskas ${ }^{*}$, W. Steyaert, D. Rots, R. Pfundt, H. G. Yntema, H. G. Brunner, A. Hoischen, L. E. L. M. Vissers, C. Gilissen

Department of Human Genetics, Radboud University Medical Center, Nijmegen, Netherlands

De novo mutations (DNMs) are an important cause of genetic disorders. The accurate identification of DNMs is therefore fundamental to rare disease research and diagnostics. Unfortunately, identifying reliable DNMs remains a major challenge due to sequence errors, uneven coverage and mapping artifacts.

Here, we developed a deep convolutional neural network (CNN) DNM caller: deNovoCNN. We trained deNovoCNN by encoding the sequence alignment reads for a trio as $164 \times 160$ resolution images. We trained our model $(80 / 20 \%$ training/validation split) on 4,868 high-quality DNMs from whole exome sequencing (WES) data of 2,018 trios with 
intellectual disability (ID). We then compared the DNM calls in 20 separate trios to those of three other de novo callers: GATK, DeNovoGear (DNG), and Radboudumc inhouse tool $(\mathrm{IH})$. The identified variants were manually inspected though IGV.

deNovoCNN outperformed other calling approaches with an accuracy of $99.38 \%$ (GATK $75.69 \%$, DNG $30.97 \%$, IH 97.54\%). Additional Sanger validation for a subset of mutations is ongoing. When applying deNovoCNN to 278 unresolved trios, we identified 5 previously missed DNMs in monoallelic ID genes (CDK13, PAFAH1B1, TCF20, SMC1A and PRKARIA), that provide a likely diagnosis based on ACMG criteria. All of these mutations were confirmed to be de novo events by Sanger sequencing.

Overall, deep convolutional neural networks are a powerful tool for DNM discovery, and have the potential to call clinically relevant DNMs missed by existing approaches.

K. Šablauskas: None. W. Steyaert: None. D. Rots: None. R. Pfundt: None. H. G. Yntema: None. H. G. Brunner: None. A. Hoischen: None. L. E. L. M. Vissers: None. C. Gilissen: None.

\section{C03.3}

GestaltMatch: breaking the limits of rare Mendelian disorder diagnosis by matching patients with nextgeneration phenotyping

\author{
T. Hsieh ${ }^{\text {* }}$, A. Bar-Haim ${ }^{2}$, G. Nadav ${ }^{2}$, T. J. Pantel ${ }^{3}$, N. \\ Fleischer $^{2}$, P. Krawitz ${ }^{1}$
}

${ }^{1}$ Institute for Genomic Statistics and Bioinformatics, Bonn, Germany, ${ }^{2}$ FDNA, Boston, MA, United States, ${ }^{3}$ Charité Universitätsmedizin Berlin, Berlin, Germany

Introduction: Recent advances in next-generation phenotyping (NGP) for syndromology such as DeepGestalt have learned phenotype representations of multiple disorders by training on thousands of patient photos. However, many Mendelian syndromes are still not represented by existing NGP tools as only handful of patients were diagnosed. We therefore propose "GestaltMatch" which utilizes the similarity among patients to identify syndromic patients by their facial gestalt in order to extend the coverage of NGP tools.

Methods: We compiled a dataset consisting of 23,000 patients with 1,500 different rare disorders. For each individual a frontal photo and the molecularly confirmed diagnosis were available. We utilized deep convolutional neural networks to train a model on patient's frontal photos, and facial embeddings were further extracted to construct a Clinical Face Phenotype Space (CFPS). Joint Bayesian was used to quantify the similarity among patients and correct the confounding effects of age and ethnicity in CFPS.
Results: The patients with similar phenotype were located in close proximity in the CFPS. Ranking syndromes by distance in CFPS, we achieved $80 \%$ top- 10 accuracy in identifying super rare Mendelian diseases that are not yet represented in DeepGestalt. Moveover, as a tool for matching patients of unknown cause, more than 100 pairs of unrelated patients were identified that show a similarity comparable to patients with the aforementioned super rare disorders, suggesting novel phenotypes of shared molecular etiology.

Conclusions: GestaltMatch opens the door to extend the coverage of syndromes recognized by NGP tools and to enable further exploration of the unknown phenotypegenotype associations.

T. Hsieh: None. A. Bar-Haim: A. Employment (full or part-time); Significant; FDNA. G. Nadav: A. Employment (full or part-time); Significant; FDNA. T. J. Pantel: None. N. Fleischer: A. Employment (full or part-time); Significant; FDNA. P. Krawitz: F. Consultant/Advisory Board; Modest; FDNA.

\section{$\mathbf{C 0 3 . 4}$}

Untranslated region (UTR) variant analysis across 71,702 genomes to build a framework for variant interpretation

S. Pajusalu ${ }^{1,2,3^{*}}$, N. J. Lake $e^{1,4}$, M. Lek ${ }^{1}$

${ }^{I}$ Department of Genetics, Yale University School of Medicine, New Haven, CT, United States, ${ }^{2}$ Department of Clinical Genetics, Institute of Clinical Medicine, University of Tartu, Tartu, Estonia, ${ }^{3}$ Department of Clinical Genetics, United Laboratories, Tartu University Hospital, Tartu, Estonia, ${ }^{4}$ Murdoch Children's Research Institute, Royal Children's Hospital, Melbourne, Australia

The importance of UTRs in the regulation of gene expression has been widely appreciated. However, UTR variants associated with Mendelian disorders have been understudied. This is due to our inability to interpret the functional impact of UTR variants and a lack of clear interpretation guidelines.

To investigate human UTR variants, we gathered $1,049,754$ single nucleotide substitutions in canonical $5^{\prime}$ UTRs and 5,289,132 in canonical $3^{\prime}$ UTRs from gnomAD v3 database. Notably, this is more than a 2-fold increase of UTR variants from the previous gnomAD release. We assessed the effects of variants affecting upstream open reading frames (uORFs), Kozak sequences, polyadenylation signals, and miRNA-binding sites. These data were used to build a publicly available variant annotation tool which is the basis of our UTR variant interpretation framework.

Using a genome-wide heptamer model of constraint enabled us to identify that UTRs show less variation than 
expected; this is even more pronounced across $5^{\prime}$ UTRs and functional elements like uORFs. By assessing UTR constraint and the absence of variants with strong regulatory effects, we were able to prioritize novel candidate disease genes. For example, MDGA1, a regulatory synaptic protein, is under strong constraint for $5^{\prime} \mathrm{UTR}$ and none of its 55 putative uORF-creating variants exist in gnomAD indicating strong negative selection.

We demonstrate the utility of the framework by classifying variants reported in the literature and databases, as well as present results from large neuromuscular disease cohorts where we have applied this framework to improve the interpretation of UTR variants.

Grant: PUTJD827

S. Pajusalu: None. N. J. Lake: None. M. Lek: None.

\section{C03.5}

Hotspot detection in homologous protein domains using de novo mutations from 31,058 patients identify candidate developmental disorder genes

L. van de Wiel $^{1,2 *}, H$. Venselaar ${ }^{2}$, L. E. L. M. Vissers ${ }^{3}$, G.

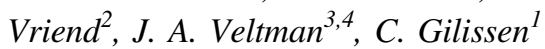

${ }^{1}$ Department of Human Genetics, Radboud Institute for Molecular Life Sciences, Radboud University Medical Center, Nijmegen, Netherlands, ${ }^{2}$ Centre for Molecular and Biomolecular Informatics, Radboud Institute for Molecular Life Sciences, Radboud University Medical Center, Nijmegen, Netherlands, ${ }^{3}$ Department of Human Genetics, Donders Institute for Brain, Cognition and Behaviour, Radboud University Medical Center, Nijmegen, Netherlands, ${ }^{4}$ Institute of Genetic Medicine, International Centre for Life, Newcastle University, Newcastle upon Tyne, United Kingdom

Identification of disease-causing variants is an ongoing effort, and requires integration of various biologically relevant prioritization strategies. We have previously shown that genetic variation can be aggregated through homologous protein domains and used to interpret variants of unknown significance. Here, we show that we can use this concept to robustly identify critical hotspots of de novo mutations (DNMs) that suggest novel disease gene candidates. We used a dataset of 45,221 DNMs from 31,058 patients with developmental disorders (DD). Of these, 15,392 DNMs locate to protein domain regions, belonging to 2,311 protein domain families across 6,910 genes. We curated this list for unique genomic positions and mapped these onto aggregated domain families. We subsequently used a binomial test to identify significantly enriched positions as domain-based hotspots. From 12,389 aggregated domain positions, we found three hotspots significantly enriched for missense DNMs, all in the "ion transport protein" (PF00520) domain family (position 96, $\mathrm{p}=3.6 \times 10^{-2} ; 102, \mathrm{p}=7.1 \times 10^{-5} ;$ and $\left.231, p=8.0 \times 10^{-6}\right)$, and no hotspots enriched for protein truncating or synonymous DNMs. The 57 missense variants driving enrichment are located in 25 genes. So far, 20 of these genes (with 52 DNMs) have been previously associated with DD $(\mathrm{p}=6.3 \times$ $\left.10^{-15}\right)$, suggesting that our approach is able to identify true disease genes. In the remaining 5 genes, genetic intolerance and molecular gene function suggest $K C N H 5, K C N G 1$, and TPCN1 as putative novel candidate genes for DD. Thus, we show a novel approach based on hotspot identification in homologous protein domains that may be used to identify candidate disease genes.

L. van de Wiel: None. H. Venselaar: None. L. E. L. M. Vissers: None. G. Vriend: None. J. A. Veltman: None. C. Gilissen: None.

\section{C03.6}

Cell type specific genetic regulation of gene expression across human tissues

S. Kim-Hellmuth ${ }^{1,2^{*}}$, F. Aguet ${ }^{3}$, M. Oliva ${ }^{4}$, S. Kasela ${ }^{2}, M$. Muñoz-Aguirre ${ }^{5}$, V. Wucher ${ }^{5}$, GTEx Consortium, R. Guigó ${ }^{5}$, B. E. Stranger ${ }^{6}, K$. G. Ardlie ${ }^{3}$, T. Lappalainen ${ }^{2,7}$

${ }^{I}$ Dr. von Hauner Children's Hospital, Department of Pediatrics, University Hospital, LMU Munich, Munich, Germany, ${ }^{2}$ New York Genome Center, New York, NY, United States, ${ }^{3}$ The Broad Institute of Massachusetts Institute of Technology and Harvard University, Cambridge, MA, United States, ${ }^{4}$ Section of Genetic Medicine, Department of Medicine, The University of Chicago, Chicago, IL, United States, ${ }^{5}$ Centre for Genomic Regulation (CRG), The Barcelona Institute for Science and Technology, Barcelona, Spain, ${ }^{6}$ Center for Genetic Medicine, Department of Pharmacology, Northwestern University, Feinberg School of Medicine, Chicago, IL, United States, ${ }^{7}$ Department of Systems Biology, Columbia University, New York, NY, United States

Introduction: The Genotype-Tissue Expression (GTEx) project has identified expression and splicing quantitative trait loci (eQTLs and sQTLs) in cis for the majority of genes across a range of human tissues. However, the interpretation of these e/sQTLs has been limited by the heterogeneity of cell type abundances in bulk tissue.

Materials and Methods: Here, we applied cell type deconvolution to characterize the cell type specificity of eQTLs and sQTLs for 43 cell type-tissue combinations, using seven cell types across 35 tissues from up to 838 individuals. 
Results: Across cell types and tissues, we detected 3,347 genes with a cell type interacting eQTL (ieQTL) and 987 genes with a cell type interacting SQTL (isQTL) at 5\% FDR. Furthermore, ieQTL-genes (ieGenes) had a larger number of independent eQTLs in standard eQTL mapping and were enriched among tissue-specific eGenes, indicating that cell type-specific regulatory activity drives cell typespecific eQTL effects that contribute to allelic heterogeneity and tissue-specificity of eQTLs. Cell type ieQTLs were also enriched in sex-biased eQTLs suggesting that these higher order $\mathrm{G} \times \mathrm{E}$ interactions are driven by cell composition changes. Finally, colocalization analysis of cell type ieQTLs and isQTLs with 87 complex traits not only pinpointed the cellular specificity of known eQTLs but identified novel colocalized loci that were masked in bulk tissue.

Conclusions: Our results emphasize the importance of identifying genetic effects on gene expression at the cellular level and demonstrate that cell type-specific effects can be uncovered in bulk tissues by integrating increasingly available single-cell and cell type-specific transcriptomic data.

S. Kim-Hellmuth: None. F. Aguet: None. M. Oliva: None. S. Kasela: None. M. Muñoz-Aguirre: None. V. Wucher: None. R. Guigó: None. B. E. Stranger: None. K. G. Ardlie: None. T. Lappalainen: None.

\section{C04 Cancer Genetics I: Basic Research in Cancer}

\section{C04.1}

Unique somatic mutation lanscape enhances tumor predisposition in the kidney proximal tubule

I. Franco ${ }^{1 *}$, H. T. Helgadottir ${ }^{l}$, A. Moggio ${ }^{1}$, M. Larsson $^{2}$, P. Vrtacnik ${ }^{l}$, A. Johansson ${ }^{3}$, N. Norgren ${ }^{4}$, P. Lundin ${ }^{5}, D$. Mas-Ponte ${ }^{6}$, J. Nordström ${ }^{7}$, T. Lundgren ${ }^{7}$, P. Stenvinkel ${ }^{7}, L$.

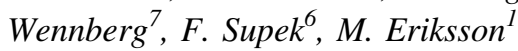

${ }^{1}$ Karolinska Institutet, HUDDINGE, Sweden, ${ }^{2}$ Science for Life Laboratories, LINKÖPING, Sweden, ${ }^{3}$ Science for Life Laboratories, UPPSALA, Sweden, ${ }^{4}$ Science for Life Laboratories, UMEA, Sweden, ${ }^{5}$ Science for Life Laboratories, STOCKHOLM, Sweden, ${ }^{6}$ Institute for Research in Biomedicine (IRB Barcelona), BARCELONA, Spain, ${ }^{7} \mathrm{Kar}$ olinska University Hospital, HUDDINGE, Sweden

Introduction: Somatic mutations accumulating in the genome of human cells can be used to track mutagen exposure and to identify cancer-prone cell types.

Materials and methods: To detect somatic mutations in single genomes from healthy, human cells, biopsies were obtained from six healthy donors undergoing kidney explant for donation. Donors' age spanned from 30 to 69 . Single cells from kidney, skin, blood, subcutaneous and visceral fat were clonally expanded and subjected to both whole genome sequencing and gene expression analyses. Multi-organ biopsies from each donor allowed wellcontrolled comparison of somatic mutation landscapes in different tissues.

Results: Somatic mutation and gene expression data identified a population of kidney tubule progenitors that showed a mutation profile similar to progenitor cells from other tissues (fat, skeletal muscle and blood) and low amount of mutations, indicating protection from mutagen exposure. Conversely, de-differentiated cells derived from damaged proximal tubules showed a mutation profile similar to the most common cancer tumor subtypes (clear cell renal cell- and papillary-carcinoma). High mutation burden and a unique mutation landscape, characterized by mutation enrichment in active chromatin, regulatory and transcribed regions, caused 6-fold faster accumulation of potential, cancer-driver mutations.

Conclusions: Our somatic mutation data support the existence of a stem cell-like population protected from mutations in the kidney tubules. Conversely, the proximal tubule of healthy, human kidneys is exposed to unique mutagenic processes that increase tumor predisposition. Fundings from Swedish Research Council, Center for Innovative Medicine, Engkvist, Hagelen, Österman and Jeanssons Foundations, Svenska Läkaresällskapet and Riksbankens Jubileumsfond

I. Franco: None. H. T. Helgadottir: None. A. Moggio: None. M. Larsson: None. P. Vrtacnik: None. A. Johansson: None. N. Norgren: None. P. Lundin: None. D. Mas-Ponte: None. J. Nordström: None. T. Lundgren: None. P. Stenvinkel: None. L. Wennberg: None. F. Supek: None. M. Eriksson: None.

\section{C04.2}

TFEB constitutive activation underlies polycystic kidney and renal carcinoma in Birt-Hogg-Dube' syndrome

\author{
C. Di Malta ${ }^{*}$, G. Napolitano, A. Esposito, M. Matarese, V. \\ Benedetti, A. Zampelli, D. Siciliano, A. Venuta, M. Cesana, \\ C. Vilardo, E. Nusco, J. Monfregola, A. Ballabio
}

Telethon Institute of Genetics and Medicine (TIGEM), Pozzuoli (NA), Italy

Loss of function mutations in the Folliculin $(F L C N)$ gene are responsible for the autosomal dominant inherited BirtHogg-Dube' (BHD) syndrome, a genetic disorder characterized by benign skin tumors, lung and kidney cysts and kidney cancers. Renal tumors represent the most severe manifestation of the disease and develop following a somatic second-hit mutation leading to the inactivation of the remaining FLCN allele. To date, the pathogenetic 
mechanisms downstream FLCN depletion are still unknown. FLCN acts a GTPase-activating protein (GAP) for the Rag family GTPase, in particular $\mathrm{RagC} / \mathrm{D}$, converting them to their active forms, which signal amino acid sufficiency to the mechanistic Target Of Rapamycin Complex 1 (mTORC1), a key regulator of cell metabolism, highly implicated in cancer. Previous studies indicated that kidney-specific Flcn deletion in mice recapitulates kidney phenotype observed in BHD patients. Here we show that loss of FLCN induces a constitutive activation of the Transcription Factor EB (TFEB), the master regulator of lysosomal biogenesis and autophagy, which drives upregulation of several target genes relevant for disease phenotype, in particular Rag $\mathrm{C} / \mathrm{D}$, thus leading to hyperactivation of mTORC1 signaling. Remarkably, depletion of Tfeb in Flcn-KO mice, which was achieved by generating $\mathrm{Flcn} / \mathrm{Tfeb}$ double $\mathrm{KO}$ mice, fully rescued kidney phenotype and associated lethality, thus pointing to TFEB as the major driver of BHD syndrome. Interestingly, increased TFEB nuclear localization is also observed in renal tumors from BHD patients, hence suggesting that targeting this transcription factor could be a valuable therapeutic strategy for the treatment of this genetic disorder.

C. Di Malta: None. G. Napolitano: None. A. Esposito: None. M. Matarese: None. V. Benedetti: None. A. Zampelli: None. D. Siciliano: None. A. Venuta: None. M. Cesana: None. C. Vilardo: None. E. Nusco: None. J. Monfregola: None. A. Ballabio: F. Consultant/Advisory Board; Modest; CASMA Therapeutics, Inc, Cambridge, MA 02139.

\section{C04.3}

Oncogenic transcription factors are over-represented in mutated active DNA binding sites in neuroblastoma and Wilms tumor

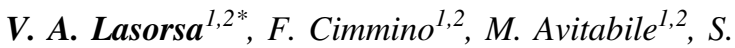 \\ Cantalupo $^{1,2}{ }^{\text {, A. Montella }}{ }^{1,2}$, B. De Angelis ${ }^{3}$, M. Morini $^{4}, C$. \\ de Torres ${ }^{5}, A$. Castellano ${ }^{3}, F$. Locatelli ${ }^{3,6}$, A. Iolascon $^{1,2}$, M. \\ Capasso $^{1,2,7}$
}

${ }^{1}$ CEINGE - Biotecnologie Avanzate, Napoli, Italy, ${ }^{2}$ Department of Molecular Medicine and Medical Biotechnology, Università degli Studi di Napoli Federico II, Napoli, Italy, ${ }^{3}$ Department of Pediatric Haematology and Oncology, IRCCS Ospedale Pediatrico Bambino Gesù, Roma, Italy, ${ }^{4}$ Laboratory of Molecular Biology, IRCCS Istituto Giannina Gaslini, Genova, Italy, ${ }^{5}$ Hospital Sant Joan de Déu, Developmental Tumor Biology Laboratory and Department of Oncology, Barcelona, Spain, ${ }^{6}$ Department of Paediatrics, Sapienza University of Rome, Roma, Italy, ${ }^{7}$ IRCCS SDN, Napoli, Italy
Introduction: The contribution of coding mutations to oncogenesis has been largely clarified whereas little is known about somatic mutations in noncoding DNA and their role in driving tumors remains controversial. Here, we used an alternative approach to interpret the functional significance of noncoding somatic mutations in promoting tumorigenesis.

Methods: Noncoding somatic mutations of 151 neuroblastomas (NB) and of 81 Wilms tumours (WT) were, in parallel, integrated with ENCODE data to locate somatic mutations in regulatory elements specifically active in tumor cells (of NB and WT), non-specifically active in tumor cells (of NB and WT), and non-active. Within these types of elements, we identified transcription factors (TFs) whose binding sites (BS) were enriched or depleted in mutations. For these TFs, a gene expression signature was built to assess their implication in NB and WT.

Results: For both tumor types, the pathogenicity of mutations was significantly higher in TFBS of regulatory elements specifically active in tumor cells, as compared to the others. Here, we found over-represented TFs mainly involved in cell cycle phase transitions $(n=18$ and $n=35$ for NB and WT, respectively), and under-represented TFs primarily regulating cell differentiation ( $n=15$ and $n=21$ for NB and WT, respectively). A gene expression signature based on over-represented TFs correlated with poor survival and unfavourable prognostic markers.

Conclusions: We propose a novel approach to study the involvement of regulatory variants in NB and WT that could be extended to other cancers and provide further evidence that alterations of gene expression may have relevant effects in tumour development.

V. A. Lasorsa: None. F. Cimmino: None. M. Avitabile: None. S. Cantalupo: None. A. Montella: None. B. De Angelis: None. M. Morini: None. C. de Torres: None. A. Castellano: None. F. Locatelli: None. A. Iolascon: None. M. Capasso: None.

\section{C04.4}

Characterization of circRNA profiles in childhood acute lymphoblastic leukemia

A. Gutierrez-Camino ${ }^{1,2 *}$, M. Caron $^{2}$, C. Richer ${ }^{2}$, E. LopezLopez $^{l}$, I. Martin-Guerrero ${ }^{1}$, B. Frutos ${ }^{1}$, P. St-Onge ${ }^{2}$, A. Bataille $^{2}$, D. Sinnett ${ }^{2,3}$

${ }^{1}$ University of the Basque Country, Leioa, Spain, ${ }^{2}$ Division of Hematology-Oncology, Sainte-Justine University Health Center, Montreal, QC, Canada, ${ }^{3}$ Department of Pediatrics, Montreal University, Montreal, QC, Canada

Childhood acute lymphoblastic leukemia (cALL) represents one of the most frequent causes of death by disease in 
children. Accurate patient diagnosis is fundamental to obtain higher cure rates. In recent years, a new type of non-coding RNAs, circRNA, has emerged as a promising biomarker for cancer diagnosis, however little is known about their roles in cALL development and treatment response. We propose to investigate and characterize a circRNAs in cALL, which might allow a more precise diagnosis and prognosis assessment, thus opening the door to better outcomes. Towards this goal we have performed a transcriptome analysis (RNA-Seq) of $156 \mathrm{cALL}$ patients (124 B-ALL and $26 \mathrm{~T}$-ALL) from two independent cohorts. CircExplorer was used to quantify, annotate and characterize circRNAs. A total of 187,504 circRNAs were identified in the discovery cohort. The size of the circRNAs was on average $820 \mathrm{bp}$, with a mean of 5 exons per circRNA. The overall expression of circRNA variants was between 1 and 452 per gene with a mean of 14,6. Unsupervised clustering analysis showed that circRNAs could properly stratify cALL subtypes. Differential circRNA expression between ALL and controls showed a total of 726 deregulated in the whole cohort (390 upregulated and 336 downregulated). The $50 \%$ of circRNAs were unique for specific subtypes. These results were validated in an independent cohort. In conclusion, we have defined circRNA characteristics in cALL and identified subtype-specific circRNAs. In addition to protein-coding genes and other noncoding RNAs, we have shown that circRNA expression profiles can accurately classify cALL subtypes.

A. Gutierrez-Camino: None. M. Caron: None. C. Richer: None. E. Lopez-Lopez: None. I. Martin-Guerrero: None. B. Frutos: None. P. St-Onge: None. A. Bataille: None. D. Sinnett: None.

\section{C04.5}

Patient-derived PDAC organoids recapitulate tumor cell state heterogeneity and functional hierarchy in vitro

T. G. Krieger ${ }^{1}$, J. Jabs ${ }^{2}$, S. LeBlanc ${ }^{3}, A$. Giri $^{l}$, O. Strobel ${ }^{3}$, R. Eils ${ }^{l}$, C. Conrad ${ }^{1}$

${ }^{1}$ Digital Health Center, Berlin Institute of Health (BIH)/ Charité-Universitätsmedizin, Berlin, Germany, ${ }^{2}$ Division of Theoretical Bioinformatics, German Cancer Research Center (DKFZ), Heidelberg, Germany, ${ }^{3}$ European Pancreas Center, Department of Surgery, Heidelberg University Hospital, Heidelberg, Germany

Pancreatic ductal adenocarcinoma (PDAC) is projected to be the second leading cause of cancer mortality by 2030 , and responses to current therapy differ markedly between patients. Transcriptomic analyses have established two subtypes of PDAC: 'classical' tumors characterised by a higher level of differentiation, and 'basal-like' tumors showing aggressive clinical behaviour. Recent single-cell sequencing studies have demonstrated that PDAC tumors comprise diverse cell populations, but the functional interplay and evolutionary hierarchy of PDAC cells remains elusive.

We derived PDAC organoids from primary tumors of 18 patients, together with two matched samples from liver metastases. By scRNA-seq, we determined that PDAC organoids consist of ductal cells with patient-specific expression of several gene groups. Organoids derived from higher-grade tumors tend to show more 'basal-like' gene expression, consistent with previous data. However, 'classical' and 'basal-like' cells may coexist within the same tumor, and the level of subtype heterogeneity within tumors is independently linked to poor prognosis.

Despite intertumor heterogeneity, we identified several tumor cell states shared across patients, including a cycling progenitor cell state and a differentiated secretory state, which are connected by a differentiation hierarchy. By integrating scRNA-seq data from PDAC organoids and primary tumors, we show that these cell states and hierarchy are mirrored within the ductal cell compartment in primary PDAC.

Our results uncover a hierarchy of functional PDAC cell states in organoids and primary tumors, supporting the use of PDAC organoids as a clinically relevant model for in vitro studies of tumor heterogeneity.

T. G. Krieger: None. J. Jabs: None. S. LeBlanc: None. A. Giri: None. O. Strobel: None. R. Eils: None. C. Conrad: None.

\section{C04.6}

Integrative analyses of 100 genomes of Basal Cell Carcinoma in the context of transcription and methylation profiles
S. I. Nikolaev ${ }^{1 *}$, A. Yurchenko ${ }^{l}$, A. Sartori ${ }^{2}$, I. Padioleau $^{l}$, F. Rajabi ${ }^{1}$, L. Parmentier ${ }^{3}$, D. Salomon ${ }^{4}$, E. Dermitzakis ${ }^{2}$, H. Ongen ${ }^{5}$

${ }^{1}$ Gustave Roussy, VILLEJUIF, France, ${ }^{2}$ Univeristy of Geneva, Geneva, Switzerland, ${ }^{3}$ Hospital of Sierre, Sierre, Switzerland, ${ }^{4}$ Seujet Dermatological Clinics, Geneva, Switzerland, ${ }^{5}$ University of Geneva, Geneva, Switzerland

Basal Cell Carcinoma (BCC) of skin is the commonest human malignancy with the lifetime risk of $30 \%$ and increasing incidence. BCC is characterized by constitutive activation of the Sonic hedgehog pathway and by hypermutator phenotype due to UV. In this study we present Whole Genome Sequences (WGS) of 96 and Whole Exome Sequences of 259 BCC. These data are complemented by RNA-Seq and Methylation profiling of BCC and adjacent skin. Over 20 million point mutations allowed 
unprecedentedly detailed analysis of UV-induced mutagenesis in skin cancer and revealed differences between $\mathrm{BCC}$ and melanoma. Variation in spectrums and clusterization of mutations across genomic regions with different sets of genomic covariates including tumor-derived transcriptomes and methylomes was used for decomposition into separate biological processes. Driver discovery, performed with multiple methods, validated known genes including PTCH1, SMO, MYCN, PTPN14 and others as well as rendered a handful of novel drivers in other cancer pathways. RNA-Seq analysis uncovered the most important pathways involved in BCC pathogenesis and histological heterogeneity. Numerous structural variants and gene fusions were retrieved using both WGS and RNA-Seq. These events as well as a list of 23 focal deletions suggested novel putative tumor suppressor genes in BCC. Functional non-coding genomic variation was assessed with somatic cis-eQTL analyses which revealed consequences of somatic mutations in promoters at the allelic expression of nearby genes including putative cancer drivers. This work represents a first large scale genomic study of BCC dissecting mutational mechanisms and functional consequences of coding and non-coding somatic mutations.

S. I. Nikolaev: None. A. Yurchenko: None. A. Sartori: None. I. Padioleau: None. F. Rajabi: None. L. Parmentier: None. D. Salomon: None. E. Dermitzakis: None. H. Ongen: None.

\section{C05 Elucidating the Function of Cardiac Genes}

\section{C05.1}

\section{Investigating the role of KMT2C in heart development}

\section{S. C. Borland", G. Tenin, R. Monaghan, S. Williams, M. Zi, C. Wilson, S. Prehar, E. Cartwright, S. Abraham, B. Keavney}

Division of Cardiovascular Sciences, University of Manchester, Manchester, United Kingdom

Histone modifiers have been implicated as candidate genes in congenital heart disease indicating they are important in heart development. Previously, our lab performed exome sequencing of 829 tetralogy of Fallot patients which identified 18 mutations in $K M T 2 C$ that are absent in gnomAD and have a combined annotation dependent depletion (CADD) score of more than 20. KMT2C is a H3K4 methyltransferase active predominantly at enhancers. Using in situ hybridisation we have shown $\mathrm{Kmt2c}$ expression during key stages of mouse heart development. Human embryonic hearts also show KMT2C expression during equivalent stages. We have studied a mouse model where the SET domain of KMT2C is deleted making the protein catalytically inactive. Homozygous deletion results in neonatal lethality. Homozygous embryos and pups with the deletion have an incompletely penetrant congenital heart defect phenotype. They have ventricular septal defects with or without an overriding aorta (as in tetralogy of Fallot). In homozygous pups and embryos the ventricular myocardium is abnormal resembling non-compaction. At day 16.5 of development the compact myocardium of the left ventricle is significantly thinner $(\mathrm{p}<0.05)$ in homozygous embryos compared to wild-type. Adult heterozygous mice have a significantly prolonged PR interval $(p=0.01)$ compared to wild-type (measured by unconscious ECG). All other electrocardiography and echocardiography parameters measured were not significantly different compared to wildtype. Our data for the first time shows that KMT2C plays a role in cardiac development and congenital heart disease. We have also identified a novel role for KMT2C in myocardium formation and cardiac function. Grant reference: BHF FS/16/58/32734

S. C. Borland: None. G. Tenin: None. R. Monaghan: None. S. Williams: None. M. Zi: None. C. Wilson: None. S. Prehar: None. E. Cartwright: None. S. Abraham: None. B. Keavney: None.

\section{C05.2}

Mutations in $K D R$, encoding for vascular endothelial growth factor receptor 2, contribute to Tetralogy of Fallot

D. Skoric-Milosavljevic ${ }^{l *}, N$. Lahrouchi ${ }^{l}, F$. Bosada ${ }^{l}, G$. Dombrowsky ${ }^{2}$, F. Tjong ${ }^{1}$, I. El Bouchikhi ${ }^{3}$, M. Hababa ${ }^{1}$, S.

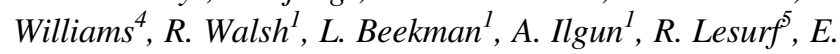
Audain $^{2}$, J. Breckpot ${ }^{6}$, B. J. Mulder ${ }^{1}$, S. Clur ${ }^{1}$, S. Mital ${ }^{5}$, B. Keavney $^{4}$, M. Hitz ${ }^{2}$, V. Christoffels ${ }^{l}$, E. M. Lodder ${ }^{l}$, A. V. Postma $^{l}$, C. R. Bezzina ${ }^{l}$

${ }^{1}$ Amsterdam University Medical Center, Amsterdam, Netherlands, ${ }^{2}$ University Medical Center Schleswig-Holstein, Kiel, Germany, ${ }^{3}$ University of Sidi Mohammed Ben Abdellah, Fez, Morocco, ${ }^{4}$ The University of Manchester, Manchester, United Kingdom, ${ }^{5}$ The Hospital for Sick Children, Toronto, ON, Canada, ${ }^{6}$ Center for Human Genetics, Catholic University Leuven, Leuven, Belgium

Introduction: Congenital heart defects (CHD) are the most common birth defects. Although there is a clear genetic component, a substantial proportion of CHD cases remain genetically unexplained. Family based studies are suitable to identify rare, highly penetrant variants in novel candidate genes.

Material and Methods: We conducted whole-exome sequencing in a family with two children affected with tetralogy of fallot (TOF). We generated and studied knock- 
in mice carrying variants homologues to two missense variants in the gene $K D R$, which likely underlie the TOF phenotype in the family. Furthermore, we screened a cohort of TOF probands of European descent for likely diseasecausing variants in $K D R$ and performed a burden test for rare genetic variants in this gene in an ancestry-matched case-control dataset.

Results: Both affected children are compound heterozygous for two missense variants in KDR (p.Gly345Trp/p. Gly537Arg), encoding vascular endothelial growth factor receptor 2. While mice that were compound heterozygous for these variants displayed no CHD, mice homozygous for the p.Gly537Arg variant displayed embryonic lethality with defective vasculogenesis and pericardial edema, mimicking the phenotype of $K d r$ knock-out mice. Compared to the gnomAD database there seems to be an enrichment of protein-truncating variants (PTVs) among a set of 1,226 TOF probands of European descent $\left(\mathrm{P}=5 \times 10^{-7}\right)$. Furthermore, we identified a significant burden of rare $(\mathrm{MAF}<0.1 \%)$ missense variants and PTVs in $K D R$ in European TOF patients versus controls $\left(\mathrm{P}=9 \times 10^{-4}\right)$.

Conclusions: Based on a family study and case-control analysis, we highlight the importance of $K D R$ in the pathogenesis of CHD in humans.

D. Skoric-Milosavljevic: None. N. Lahrouchi: None. F. Bosada: None. G. Dombrowsky: None. F. Tjong: None. I. El Bouchikhi: None. M. Hababa: None. S. Williams: None. R. Walsh: None. L. Beekman: None. A. Ilgun: None. R. Lesurf: None. E. Audain: None. J. Breckpot: None. B. J. Mulder: None. S. Clur: None. S. Mital: None. B. Keavney: None. M. Hitz: None. V. Christoffels: None. E. M. Lodder: None. A. V. Postma: None. C. R. Bezzina: None.

\section{C05.3}

Bi-allelic loss-of-function mutations in PLD1 cause congenital right-sided cardiac valve defects and neonatal cardiomyopathy

N. Lahrouchi ${ }^{*}$, A. Postma ${ }^{2}$, C. M. Salazar ${ }^{3}$, D. De

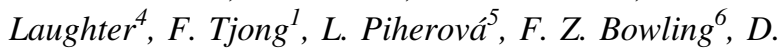
Zimmerman $^{1}$, E. M. Lodder ${ }^{1}$, A. Ta-Shma ${ }^{7}$, Z. Perles ${ }^{7}$, L. Beekman $^{1}$, A. Ilgun ${ }^{8}$, Q. Gunst ${ }^{8}$, M. Hababa ${ }^{1}$, D. ŠkorićMilosavljević $^{1}, P$. de Knijff, R. de Leeuw ${ }^{9}, J$. Y. Robinson ${ }^{10}$, S. C. Burn ${ }^{11}$, H. Mustafa ${ }^{11}$, M. Ambrose ${ }^{12}$, T. Moss ${ }^{13}$, J. Jacober $^{14}$, D. M. Niyazov ${ }^{14}$, A. Rousounides ${ }^{15}$, A. AristidouKallika $^{16}$, G. Tanteles $^{17}$, M. Magliozzi ${ }^{18}$, F. C. Radio ${ }^{19}, G$. W. E. van Santen ${ }^{20}$, J. C. Herkert ${ }^{21}$, O. Elpeleg ${ }^{22}$, M. van den Hoff', B. Mulder ${ }^{1}$, M. V. Airola ${ }^{6}$, S. Kmoch ${ }^{5}$, J. V. Barnett $^{10}$, S. Clur ${ }^{23}$, M. A. Frohman ${ }^{3}$, C. R. Bezzina ${ }^{1}$

${ }^{1}$ Amsterdam UMC, University of Amsterdam, Heart Center; Department of Clinical and Experimental Cardiology, Amsterdam Cardiovascular Sciences, Amsterdam,
Netherlands, ${ }^{2}$ Department of Clinical Genetics, Amsterdam UMC, Amsterdam, Netherlands, ${ }^{3}$ Department of Pharmacological Sciences and Graduate Program in Molecular and Cellular Pharmacology, Stony Brook University, Stony Brook, New York, NY, United States, ${ }^{4}$ Department of Cell and Developmental Biology, Vanderbilt University School of Medicine, Nashville, TN, United States, ${ }^{5}$ Research Unit for Rare Diseases, Department of Pediatrics and Adolescent Medicine, 1st Faculty of Medicine, Charles University and General University Hospital, Prague, Czech Republic, ${ }^{6}$ Department of Biochemistry and Cell Biology, Stony Brook University, Stony Brook, New York, NY, United States, ${ }^{7}$ Department of Pediatric Cardiology, Hadassah, Hebrew University Medical Center, Jerusalem, Israel, ${ }^{8}$ Department of Medical Biology, Amsterdam UMC, Amsterdam, Netherlands, ${ }^{9}$ Department of Human Genetics, Leiden University Medical Centre, Leiden, Netherlands, ${ }^{10}$ Department of Pharmacology, Vanderbilt University School of Medicine, Nashville, TN, United States, ${ }^{11}$ University of Minnesota, Department of Obstetrics, Gynecology \& Women's Health, Minneapolis, MN, United States, ${ }^{12}$ University of Minnesota, Department of Pediatrics, Division of Pediatric Cardiology, Minneapolis, MN, United States, ${ }^{13}$ University of Minnesota, Department of Pediatrics, Minneapolis, MN, United States, ${ }^{14}$ Department of Pediatrics, Ochsner Clinic, Tulane University, University of Queensland, New Orleans, LA, United States, ${ }^{15}$ Makarios Medical Centre, Nicosia, Cyprus, ${ }^{16}$ Ultrasound and Fetal Medicine Diagnostic Centre, Nicosia, Cyprus, ${ }^{17}$ Cyprus School of Molecular Medicine, Nicosia, Cyprus, ${ }^{18}$ Genetic and Rare Disease Research Division, Bambino Gesù Children's Hospital IRCCS, Rome, Italy, ${ }^{19}$ Genetic and Rare Disease Research Division, Bambino Gesù Children's Hospital IRCC, Rome, Italy, ${ }^{20}$ Department of Human Genetics, Leiden University Medical Center, Leiden, Netherlands, ${ }^{21}$ University of Groningen, University Medical Center Groningen, Department of Genetics, Groningen, Netherlands, ${ }^{22}$ Monique and Jacques Roboh Department of Genetic Research, Hadassah, Hebrew University Medical Center, Jerusalem, Israel, ${ }^{23}$ Department of Pediatric Cardiology, Emma Children's Hospital, Amsterdam UMC, University of Amsterdam, Amsterdam, Netherlands

Introduction: Mutations in PLD1, which encodes phospholipase D1, were recently associated with severe congenital cardiac valve defects in two families. However, the consequences of the mutations on PLD1 protein function and the mechanism by which PLD1 dysfunction leads to congenital valve abnormalities remained unknown.

Methods: We established an international cohort of patients with autosomal-recessive mutations in PLD1. We assessed the location of the disease-causing missense mutations on the 3-dimensional structure of PLD1 and tested 
their effect on PLD1 enzymatic activity in a cellular assay. To investigate the involvement of PLDI in endocardialmesenchymal transition (EndoMT) we used an in vitro assay to asses EndoMT in atrioventricular cushion explants.

Results: We identified 26 patients with bi-allelic PLD1 mutations. While most patients presented with right-sided congenital cardiac valve defects (24/26), two patients displayed isolated severe fetal/neonatal cardiomyopathy. Using haplotype analyses, we established that p.I668F is a founder mutation among the Ashkenazi-Jews (AJS, minorallele frequency in gnomAD-ASJ $=2 \%$ ). We found that disease-causing PLD1 missense mutations cluster in regions of the protein critical for catalytic activity. Correspondingly, a drastic reduction in enzymatic activity was observed for the mutant proteins. We demonstrate that PLD1 inhibition decreases EndoMT, an established pivotal early step in valvulogenesis.

Conclusion: We here provide the genetic and phenotypic characterization of the largest cohort of PLD1 patients. Genotyping of the p.I668F founder mutation using population-based carrier screening may be justified to assess risk of cardiac defects among ASJ-descendants. Finally, our results demonstrate that functional testing is useful for clinical interpretation of variants in PLD1.

N. Lahrouchi: None. A. Postma: None. C. M. Salazar: None. D. De Laughter: None. F. Tjong: None. L. Piherová: None. F. Z. Bowling: None. D. Zimmerman: None. E. M. Lodder: None. A. Ta-Shma: None. Z. Perles: None. L. Beekman: None. A. Ilgun: None. Q. Gunst: None. M. Hababa: None. D. Škorić-Milosavljević: None. P. de Knijff: None. R. de Leeuw: None. J. Y. Robinson: None. S. C. Burn: None. H. Mustafa: None. M. Ambrose: None. T. Moss: None. J. Jacober: None. D. M. Niyazov: None. A. Rousounides: None. A. Aristidou-Kallika: None. G. Tanteles: None. M. Magliozzi: None. F. C. Radio: None. G. W. E. van Santen: None. J. C. Herkert: None. O. Elpeleg: None. M. van den Hoff: None. B. Mulder: None. M. V. Airola: None. S. Kmoch: None. J. V. Barnett: None. S. Clur: None. M. A. Frohman: None. C. R. Bezzina: None.

\section{C05.4}

Pathogenic variants in THSD4, encoding the ADAMTSLike 6 protein, predispose to inherited thoracic aortic aneurysm

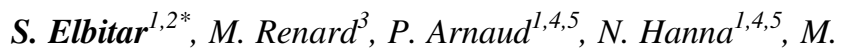

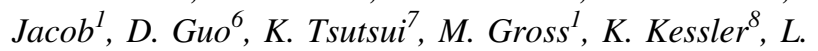
Tosolini $^{1}$, V. Dattilo ${ }^{9}$, S. Dupont ${ }^{1}$, J. Jonquet ${ }^{1}$, M.

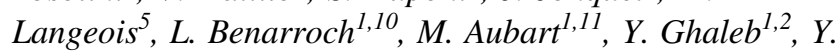
Abou Khalil ${ }^{1,2}$, M. Varret $^{1}$, P. El Khoury ${ }^{1,2}$, B. Ho-TinNoé ${ }^{1}$, Y. Alembik ${ }^{12}$, S. Gaertner ${ }^{13}$, B. Isidor ${ }^{14}$, L. Gouya ${ }^{5}$, O. Milleron $^{5}$, K. Sekiguchi ${ }^{7}$, D. Milewicz ${ }^{6}$, J. De Backer ${ }^{3}$, C. Le

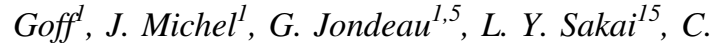
Boileau $^{1,4,5}$, M. Abifadel ${ }^{1,2}$

${ }^{1}$ Laboratory for Vascular Translational Science, INSERM U1148, Université de Paris, Centre Hospitalo-Universitaire Xavier Bichat, Paris, France, ${ }^{2}$ Laboratory of Biochemistry and Molecular Therapeutics, Faculty of Pharmacy, SaintJoseph University, Beirut, Lebanon, ${ }^{3}$ Center for Medical Genetics, Ghent University, Ghent, Belgium, ${ }^{4}$ Département de Génétique, Centre Hospitalo-Universitaire Xavier Bichat, Paris, France, ${ }^{5}$ Hôpital Bichat, Centre de Référence Maladies Rares, Syndrome de Marfan et pathologies apparentées, Assistance Publique-Hôpitaux de Paris, Paris, France, ${ }^{6}$ Department of Internal Medicine, McGovern Medical School, University of Texas Health Science Center, Houston, TX, United States, ${ }^{7}$ Institute for Protein Research, Osaka University, Suita, Osaka, Japan, ${ }^{8}$ Centre for Evolution and Cancer, Division of Molecular Pathology, Division of Cancer Therapeutics, The Institute of Cancer Research, London, United Kingdom, ${ }^{9}$ IRCCS Istituto Centro San Giovanni di Dio Fatebenefratelli, Brescia, Italy, ${ }^{10}$ Inserm UMRS_974, Centre de recherche en myologie, G. H. Pitié-Salpétrière, 47 boulevard de l'Hôpital, Paris, France, ${ }^{11}$ Service de Neuropédiatrie, Hôpital Necker-Enfants-Malades, 149 rue de Sèvres, Paris, France, ${ }^{12}$ Department of Clinical Genetic, Hôpitaux Universitaires de Strasbourg, 67091, Strasbourg, France, ${ }^{13}$ Department of Hypertension, Vascular Diseases and Pharmacology, University of Strasbourg, Strasbourg, France, ${ }^{14}$ Service de Génétique Médicale, Hôpital HôtelDieu, Centre Hospitalier Universitaire de Nantes, 44093, Nantes, France, ${ }^{15}$ Shriners Hospital for Children, Molecular \& Medical Genetics and Biochemistry \& Molecular Biology, Oregon Health \& Science University, Portland, OR, United States

Thoracic aortic aneurysm and dissection (TAAD) is a lifethreatening disease with often unrecognized inherited forms (hTAAD). Although pathogenic variants have been found in several genes, the majority of hTAAD cases are of unknown etiology. We identified in probands and families with TAAD, five functional variants in THSD4 of which two heterozygous variants leading to a premature termination codon. THSD4 encodes ADAMTSL6 (member of the ADAMTS/L superfamily), a microfibril-associated protein that promotes fibrillin-1 matrix assembly. The THSD4 variants studied lead to haplo-insufficiency or impaired assembly of fibrillin-1 microfibrils. $T h s d 4^{+/-}$mice showed progressive dilatation of the thoracic aorta. Histologic examination of aortic samples from a patient carrying a THSD4 variant and from $T h s d 4^{+/-}$mice, revealed typical medial degeneration and diffuse disruption of extracellular matrix. These findings highlight the role of ADAMTSL6 in 
aortic physiology and TAAD pathogenesis. They will improve genetic screening for hTAAD and could help develop new targeted therapies.

S. Elbitar: None. M. Renard: None. P. Arnaud: None. N. Hanna: None. M. Jacob: None. D. Guo: None. K. Tsutsui: None. M. Gross: None. K. Kessler: None. L. Tosolini: None. V. Dattilo: None. S. Dupont: None. J. Jonquet: None. M. Langeois: None. L. Benarroch: None. M. Aubart: None. Y. Ghaleb: None. Y. Abou Khalil: None. M. Varret: None. P. El Khoury: None. B. Ho-Tin-Noé: None. Y. Alembik: None. S. Gaertner: None. B. Isidor: None. L. Gouya: None. O. Milleron: None. K. Sekiguchi: None. D. Milewicz: None. J. De Backer: None. C. Le Goff: None. J. Michel: None. G. Jondeau: None. L. Y. Sakai: None. C. Boileau: None. M. Abifadel: None.

\section{C05.5}

Functional characterization of rare $\mathrm{SHOX} 2$ variants identified in sinus node dysfunction and atrial fibrillation

S. Hoffmann ${ }^{1,2 *}$, C. Paone ${ }^{3}$, S. A. Sumer ${ }^{1,2}$, S. Diebold ${ }^{3}$, B. Weiss $^{I}$, R. Roeth ${ }^{l}$, S. Clauss ${ }^{4,5}$, I. Klier ${ }^{4,5}$, S. Kääb ${ }^{4,5}$, A. Schulz, P. S. Wild ${ }^{6}$, A. Ghrib ${ }^{7,8}$, T. Zeller ${ }^{7,8}$, R. B. Schnabel $^{7,8}$, S. Just ${ }^{3}$, G. A. Rappold ${ }^{1,2}$

${ }^{I}$ Department of Human Molecular Genetics, Institute of Human Genetics, University of Heidelberg, Heidelberg, Germany, ${ }^{2}$ DZHK (German Centre for Cardiovascular Research), Partner site Heidelberg/Mannheim, Heidelberg, Germany, ${ }^{3}$ Department of Internal Medicine II, University of Ulm, Ulm, Germany, ${ }^{4}$ Department of Medicine I, Klinikum Grosshadern, University of Munich (LMU), Munich, Germany, ${ }^{5}$ DZHK (German Centre for Cardiovascular Research), Partner site Munich, Munich, Germany, ${ }^{6}$ Preventive Cardiology and Preventive Medicine, Center for Cardiology, University Medical Center of the Johannes Gutenberg-University Mainz, Mainz, Germany, ${ }^{7}$ Department of General and Interventional Cardiology, University Heart Center Hamburg (UHZ), University Hospital Hamburg/Eppendorf, Hamburg, Germany, ${ }^{8}$ DZHK (German Centre for Cardiovascular Research), Partner site Hamburg/Kiel/Luebeck, Hamburg, Germany

Sinus node dysfunction (SND) and atrial fibrillation (AF) often coexist; however, the molecular mechanisms linking both conditions remain elusive. Mutations in the homeobox-containing SHOX2 gene have been recently associated with early-onset and familial AF. Shox 2 is a key regulator of sinus node development, and its deficiency leads to bradycardia, as demonstrated in animal models. We investigated $\mathrm{SHOX} 2$ as a susceptibility gene for SND and AF by screening 98 SND patients and 450 individuals with AF. The functional relevance of the novel mutations was investigated in vivo and in vitro. A heterozygous missense mutation (p.P33R) was identified in the SND cohort and four heterozygous variants (p.G77D, p.L129=, p.L130F, p. $\mathrm{A} 293=)$ in the AF cohort. Overexpression of the pathogenic predicted mutations in zebrafish revealed pericardial edema for p.G77D, whereas the p.P33R and p.A293= variants showed no effect. In addition, a dominant-negative effect with reduced heart rates was detected for p.G77D. In vitro reporter assays demonstrated for both missense variants p.P33R and $p$. G77D significantly impaired transactivation activity. Also, a reduced Bmp4 target gene expression was revealed in zebrafish hearts upon overexpression of the p.P33R mutant. This study associates rare variants in the $\mathrm{SHOX} 2$ gene implicated in the susceptibility to arrhythmias and allows frequency estimations in an AF cohort (3/990). We also demonstrate for the first time a genetic link between SND and AF involving SHOX2. This work was supported by grants from the DFG (RA 380/14-4) and DZHK (81X2500107).

S. Hoffmann: None. C. Paone: None. S. A. Sumer: None. S. Diebold: None. B. Weiss: None. R. Roeth: None. S. Clauss: None. I. Klier: None. S. Kääb: None. A. Schulz: None. P. S. Wild: None. A. Ghrib: None. T. Zeller: None. R. B. Schnabel: None. S. Just: None. G. A. Rappold: None.

\section{C06 Sensory Disorders}

\section{C06.2}

Whole Exome Sequencing, Molecular Assays, Immunohistology and Animal Models associate USP48 to Hereditary Hearing Loss

S. Bassani ${ }^{1 *}$, A. Morgan ${ }^{2}$, M. Cocca ${ }^{3}, N$. Voisin $^{4}, J$.

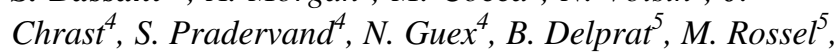
H. Locher ${ }^{6}$, E. Van Beelen ${ }^{6}$, M. Tangui ${ }^{5}$, P. Gasparini ${ }^{2,3}$, G. Girotto $^{2,3}$, A. Reymond ${ }^{4}$

${ }^{1}$ University of Lausanne, Laussane, Switzerland, ${ }^{2}$ Department of Medicine, Surgery and Health Sciences, University of Trieste, Trieste, Italy, ${ }^{3}$ IRCCS Materno Infantile Burlo Garofolo, Trieste, Italy, ${ }^{4}$ Centre for Integrative Genomics, University of Lausanne, Lausanne, Switzerland, ${ }^{5} M M D N$, University of Montpellier, EPHE-INSERM U1198, Montpellier, France, ${ }^{6}$ Department of ORL-HNS, Leiden University Medical Center, Leiden, Netherlands

Non-Syndromic Hearing Loss (NSHL) is a sensorineural disorder with high genetic heterogeneity and 115 genes already associated. We identified through exome sequencing and data aggregation an Italian family with six affected individuals and two unrelated NSHL Dutch patients with predicted-to-be deleterious missense variants in USP48. We uncovered a ninth patient presenting unilateral cochlear nerve aplasia and a de novo splice variant in the same gene. USP48 
encodes a ubiquitin carboxyl-terminal hydrolase under evolutionary constraints according to GnomAD (pLI=1; Zmissense $=4.37$ ). Pathogenicity of the patients' missense variants is supported by the three-dimensional representation of the encoded peptide. For example, the Italian missense variant affects a flexible loop that controls binding to ubiquitin thus leading to an incapacity to hydrolyze tetra-ubiquitin in our in vitro assays. Consistent with a contribution of USP48 to auditory function, immunohistology showed that the encoded protein is expressed in human hair cells/stereocilia of the utricle, the dark cell area, the Reissner's membrane, the stria vascularis and the developing organ of Corti. Engineered zebrafishes knocked-out for the USP48 paralog presented with delayed primary motoneurons development, decreased swimming velocity and circling swimming behavior indicative of vestibular dysfunction and hearing impairment. Corroboratingly, acoustic startle response assays revealed a reduced auditory response of zebrafish lacking usp 48 with a significant decrease at $600 \mathrm{~Hz}$ and $800 \mathrm{~Hz}$ frequencies, highlighting the role of this gene in auditory function. In conclusion, we describe a novel NSHL gene through a multipronged approach combining Exome Sequencing, Animal Modelization, Immunohistology and Molecular Assays.

S. Bassani: None. A. Morgan: None. M. Cocca: None. N. Voisin: None. J. Chrast: None. S. Pradervand: None. N. Guex: None. B. Delprat: None. M. Rossel: None. H. Locher: None. E. Van Beelen: None. M. Tangui: None. P. Gasparini: None. G. Girotto: None. A. Reymond: None.

\section{C06.3}

Ablation of the congenital microcoria (MCOR) critical region on 13q32.1 activates common-type glaucoma signaling pathways challenging a developmental etiology of MCOR-associated glaucoma.

C. Angee ${ }^{1 *}$, B. Nedelec ${ }^{1}$, P. David ${ }^{2}$, S. Gerber ${ }^{1}$, S. Crippa ${ }^{3}$, B. Passet ${ }^{4}$, J. Vilotte ${ }^{4}$, N. Chassaing ${ }^{5}$, J. Kaplan', C.

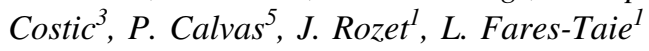

${ }^{1}$ Imagine Institute - Laboratory of Genetics in Ophtalmology (LGO), Paris, France, ${ }^{2}$ Imagine Institute - Transgenesis Platform, Paris, France, '3ules Gonin Eye Hospital, Lausanne, Switzerland, ${ }^{4}$ INRA - Animal Models for the Differentiation of Tissue (MoDiT), Jouy-en-Josas, France, ${ }^{5}$ Purpan Hospital, Toulouse, France

The iris is essential to controlling the intraocular pressure, the elevation of which leads to glaucoma. Accordingly, glaucoma is frequent in individuals with irido-dysgenesis, including congenital microcoria (MCOR), a rare dominant disease of dilator muscle (DM) development (>30\%). MCOR is due to deletions within a $1 \mathrm{Mb}$ TAD on 13q32. 1 . We generated deletions at/or around the $35-\mathrm{Kb}$ critical deletion (CD) in the mouse to investigate the regulatory architecture of the region and its role in iris development and glaucoma. Heterozygous mice carrying the CD developed normally, except for moderate reduction in pupil diameter $(\mathrm{p}<0.01)$. RNAseq revealed ectopic expression of Sox21, downstream of the CD. Other genes within the TAD were unaffected. Smaller deletions encompassing CTCFinsulators within the CD did not alter Sox 21 regulation, suggesting adoption of a nearby enhancer by Sox 21 promotor in CD mice. ChIPseq-H3K27Ac identified two enhancers of Dct involved in melanin synthesis, at the $5^{\prime}$ boundary of the TAD. Consistent with a role of Dct enhancer(s), immunohistology detected Sox 21 in the pigmented epithelium (PE). It was undetectable in the anterior layer where the DM develops, but ChIPseq identified a trophic factor among Sox 21 targets, the mRNA and product of which were elevated in the iris and aqueous humor $(\mathrm{AH})$ of CD mice as determined by RNAseq $(\mathrm{p}<0.01)$ and ELISA $(\mathrm{p}<0.01)$, respectively. Knowing that $\mathrm{AH}$ elevation of this factor is associated with primary open-angle glaucoma (POAG), we suggest that both DM malformation and glaucoma in MCOR are secondary to Sox21-mediated activation of a POAG paracrine signaling in the PE.

C. Angee: None. B. Nedelec: None. P. David: None. S. Gerber: None. S. Crippa: None. B. Passet: None. J. Vilotte: None. N. Chassaing: None. J. Kaplan: None. C. Costic: None. P. Calvas: None. J. Rozet: None. L. FaresTaie: None.

\section{C06.4}

Mouse and human studies support a role for $\mathrm{CAPN15}$ variants in cataract and microphthalmia

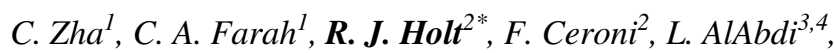 A. O. Khan ${ }^{4,5,6}$, R. Helaby, F. S. Alkuray ${ }^{4,7}$, A. Kraus $^{8}$, N. K. Ragge R.9 $^{2, \text { W. S. Sossin }}{ }^{1}$

${ }^{1}$ McGill University, Montreal, QC, Canada, ${ }^{2}$ Oxford Brookes University, Oxford, United Kingdom, ${ }^{3}$ King Saud University, Riyadh, Saudi Arabia, ${ }^{4}$ King Faisal Specialist Hospital and Research Center, Riyadh, Saudi Arabia, ${ }^{5}$ Eye Institute, Cleveland Clinic Abu Dhabi, Abu Dhabi, United Arab Emirates, ${ }^{6}$ Cleveland Clinic Lerner College of Medicine at Case Western University, Cleveland, $\mathrm{OH}$, United States, ${ }^{7}$ Alfaisal University, Riyadh, Saudi Arabia, ${ }^{8}$ Leeds General Infirmary, Leeds, United Kingdom, ${ }^{9}$ West Midlands Regional Clinical Genetics Service and Birmingham Health Partners, Birmingham Women's and Children's NHS Foundation Trust, Birmingham, United Kingdom

Introduction: Anophthalmia, microphthalmia and coloboma (AMC) are genetically heterogeneous developmental 
eye anomalies, with variants in over 100 genes implicated. However, only $25-60 \%$ of affected individuals are genetically diagnosed, depending on the phenotype. Calpains are a group of intracellular cysteine proteases important in development. Calpain 15 (CAPN15) is located at chromosome $16 \mathrm{p} 13.3$, a region previously implicated in an individual with cataracts and microphthalmia. We describe a Capn15 mouse knockout with cataract and microphthalmia, and three human families with developmental eye anomalies and CAPN15 recessive variants.

Materials and Methods: A mouse Capn15 knockout was generated using embryonic stem cells from the International Mouse Phenotyping Consortium. Variants in human AMC cases were identified through whole exome sequencing of 55 individuals from a UK patient cohort, examination of the Deciphering Developmental Disorders Study cohort, and from a clinical diagnostic laboratory.

Results: During mouse development Capn 15 was widely expressed throughout the central nervous system, including in the brain and eyes. Capn15 knockout mice presented with a significant growth deficit and a range of structural eye disorders, including cataract (31\%), microphthalmia (26\%) and anophthalmia (18\%). Three human cases with phenotypes including growth delay (2/3), cataracts $(1 / 3)$, coloboma (2/3) and microphthalmia (2/3) were identified with homozygous or compound heterozygous likely pathogenic variants in CAPN15.

Conclusions: We present evidence that variants in CAPN15 are associated with AMC. Testing for variants in this gene can be used to enhance AMC patient diagnosis, and meets criteria for clinical diagnostic testing, including those of Clinical Genome Resource (ClinGen) and Genomics England.

C. Zha: None. C. A. Farah: None. R. J. Holt: None. F. Ceroni: None. L. AlAbdi: None. A. O. Khan: None. R. Helaby: None. F. S. Alkuray: None. A. Kraus: None. N. K. Ragge: None. W. S. Sossin: None.

\section{C06.5}

\section{DYNC2H1 hypomorphic or retina-predominant variants} cause non-syndromic retinal degeneration

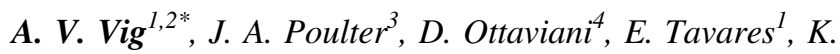
Toropova ${ }^{5}$, A. M. Tracewska, ${ }^{6,7}$, A. Mollica ${ }^{1}$, J. Kang $^{1}$, O. $K_{\text {Kehelwathugoda }}^{l}$, T. Paton ${ }^{1,8}$, J. T. Maynes ${ }^{1}$, G. Wheway ${ }^{9}$, G. Arno ${ }^{4,10}$, K. N. Khan ${ }^{3}$, M. McKibbin ${ }^{3}$, C. Toomes ${ }^{3}$, M.

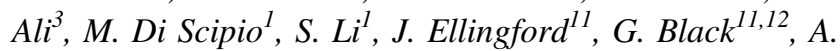
Webster $^{4,10}$, M. Rydzanicz ${ }^{7}$, P. Stawiński ${ }^{7}$, R. Ptoski ${ }^{7}, A$. Vincent $^{1,2}$, M. E. Cheetham ${ }^{4}$, C. F. Inglehearn ${ }^{3}$, A. Roberts ${ }^{5}$, E. Heon ${ }^{1,2}$

${ }^{1}$ The Hospital for Sick Children, Toronto, ON, Canada, ${ }^{2}$ The University of Toronto, Toronto, ON, Canada, ${ }^{3} \mathrm{St}$
James' University Hospital, Leeds, United Kingdom, ${ }^{4}$ UCL Institute of Ophthalmology, London, United Kingdom, ${ }^{5}$ Birbeck University of London, London, United Kingdom, ${ }^{6}$ PORT Polish Center for Technology Development, Wroclaw, Poland, ${ }^{7}$ Medical University of Warsaw, Warsaw, Poland, ${ }^{8}$ The Centre for Applied Genomics, Toronto, ON, Canada, ${ }^{9}$ University of Southampton, Toronto, ON, Canada, ${ }^{10}$ Moorfields Eye Hospital, London, United Kingdom, ${ }^{11}$ The University of Manchester, Manchester, United Kingdom, ${ }^{12}$ Saint Mary's Hospital, Manchester, United Kingdom

Introduction: The cytoplasmic dynein-2, heavy chain 1 gene $(D Y N C 2 H 1)$ encodes a motor protein important for retrograde ciliary intraflagellar transport. $\mathrm{DYNC} 2 \mathrm{Hl}$ variants have been associated with severe syndromic conditions such as Jeune asphyxiating thoracic dystrophy (JATD), with rare retinal involvement.

Material and Methods: Genome and exome sequencing were performed for 5 unrelated cases of IRD with no identified variant. In vitro assays were developed to validate the variants identified; fibroblast ciliation assay, iPSCderived retinal organoids, and an in vitro dynein motility assay.

Results: Four novel DYNC2H1 variants (V1, g.103327020_103327021dup; V2, g.103055779 A>T; V3, g.103112272 C>G; V4, g.103070104 A>C) and one previously reported (V5, g.103339363 T>G) were identified. V1 introduced a premature termination codon (PTC), whereas V2 disrupted the exon 41 splice donor site causing incomplete skipping of exon 41 . V1 and V2 impaired dynein-2 motility in vitro and perturbed IFT88 distribution within cilia. V3 is predicted to cause a PTC in a retinapredominant transcript. Analysis of retinal organoids showed that expression of this transcript increased with organoid differentiation.

Conclusions: The $\mathrm{DYNC} 2 \mathrm{Hl}$ variants discussed were either hypomorphic or affect a retina-predominant transcript. This is the first report of dynein variants, specifically DYNC2H1 variants, causing non-syndromic IRD.

Funding: Foundation Fighting Blindness Canada and USA, University of Toronto McLaughlin Centre WGS Initiative, Henry Brent Chair in Innovative Pediatric Ophthalmology Research, Brenda Eye Research Fund, Fight For Sight UK, NIH Research, Moorfields Eye Charity, Wellcome Trust, Cancer Research UK, Medical Research Council, Retina UK, IRDC Leeds and Manchester, National Science Center Poland, Royal Society, BBSRC.

A. V. Vig: None. J. A. Poulter: None. D. Ottaviani: None. E. Tavares: None. K. Toropova: None. A. M. Tracewska: None. A. Mollica: None. J. Kang: None. O. Kehelwathugoda: None. T. Paton: None. J. T. Maynes: None. G. Wheway: None. G. Arno: None. K. N. Khan: F. Consultant/ 
Advisory Board; Modest; Advisory board fees from MeiraGTx, Advisory board fees from Roche. M. McKibbin: B. Research Grant (principal investigator, collaborator or consultant and pending grants as well as grants already received); Modest; Educational travel grant from Novartis, Educational travel grant from Bayer, Educational travel grant from Allegran. C. Other Research Support (supplies, equipment, receipt of drugs or other in-kind support); Modest; Personal research funding from Alcon, Personal research funding from Roche. F. Consultant/Advisory Board; Modest; Lecture or advisory board feed from Novartis, Lecture or advisory board feed from Bayer. C. Toomes: None. M. Ali: None. M. Di Scipio: None. S. Li: None. J. Ellingford: None. G. Black: None. A. Webster: None. M. Rydzanicz: None. P. Stawiński: None. R. Płoski: None. A. Vincent: F. Consultant/Advisory Board; Modest; Adverum Technologies. M. E. Cheetham: None. C. F. Inglehearn: None. A. Roberts: None. E. Heon: F. Consultant/Advisory Board; Modest; Deep Genomics, Sanofi.

\section{C06.6}

Proteasome subunit PSMC3 variants cause neurosensory syndrome combining deafness and cataract due to proteotoxic stress

A. Kröll-Hermi ${ }^{1}$, F. Ebstein ${ }^{2}$, C. Stoetzel ${ }^{1}$, V. Geoffroy ${ }^{1}$, E. Schaefer $^{l}$, S. Scheidecker ${ }^{1}$, S. Bär ${ }^{3}$, M. Takamiya ${ }^{4}, K$. Kawakami $^{5}$, B. Zieba ${ }^{2}$, F. Studer ${ }^{6}$,V. Pelletier ${ }^{6}$, C. Speeg-

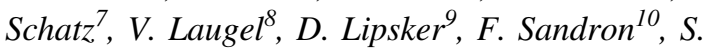
McGinn ${ }^{10}$, A. Boland ${ }^{10}$, J. Deleuze , L. Kuhn $^{11}$, J.

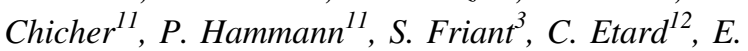
Krüger $^{2}$, J. Muller ${ }^{1 *}$, U. Strähle ${ }^{4}, H$. Dollfus ${ }^{1}$

${ }^{1}$ U1112, Strasbourg, France, ${ }^{2}$ Institut für Medizinische Biochemie und Molekularbiologie (IMBM), Greifswald, Germany, ${ }^{3}$ Laboratoire de Génétique Moléculaire, Génomique, Microbiologie (GMGM), UMR7156, Centre National de Recherche Scientifique (CNRS), Université de Strasbourg, Strasbourg, France, Strasbourg, France, ${ }^{4}$ Karlsruhe Institute of Technology (KIT), Institute of Toxicology and Genetics (ITG), Eggenstein-Leopoldshafen, Germany., Karlsruhe, Germany, ${ }^{5}$ Laboratory of Molecular and Developmental Biology, National Institute of Genetics, and Department of Genetics. SOKENDAI (The Graduate University for Advanced Studies), Mishima, Japan, Mishima, Japan, ${ }^{6}$ Centre de Référence pour les affections rares en génétique ophtalmologique, CARGO, Filière SENSGENE, Hôpitaux Universitaires de Strasbourg, Strasbourg, France, Strasbourg, France, ${ }^{7}$ Department of Ophthalmology, Hôpitaux universitaires de Strasbourg, Strasbourg, France, Strasbourg, France, ${ }^{8}$ Service de Pédiatrie, Hôpitaux Universitaires de Strasbourg, 1 avenue Molière, Strasbourg, France, Strasbourg, France, ${ }^{9}$ Faculté de
Médecine, Université de Strasbourg et Clinique Dermatologique, Hôpitaux Universitaires, Strasbourg, France., Strasbourg, France, ${ }^{10}$ Centre National de Recherche en Génomique Humaine (CNRGH), Institut de Biologie François Jacob, CEA, Université Paris-Saclay, F-91057, Evry, France., Evry, France, ${ }^{11}$ Institut de Biologie Moléculaire et Cellulaire (IBMC), Plateforme Protéomique Strasbourg-Esplanade, CNRS FRC1589, 67084 Strasbourg, France, Strasbourg, France, ${ }^{12}$ Karlsruhe Institute of Technology (KIT), Institute of Toxicology and Genetics (ITG), Eggenstein-Leopoldshafen, Germany., Strasbourg, France

Patients with a very rare association of early-onset cataracts and severe deafness as part of a neurological, sensorial and cutaneous novel syndrome were explored by Whole Genome Sequencing in a very large family. A unique deep intronic homozygous variant was identified in the PSMC3 gene (encoding the proteasome ATPase subunit Rpt5) leading to the transcription of a cryptic exon. The ubiquitinproteasome system represents the most important nonlysosomal cellular protein degradation machinery. By way of the $26 \mathrm{~S}$ proteasome as the degradative enzyme complex, it is tightly connected with the ubiquitin-dependent timely degradation of regulatory proteins involved in signalling cascades and the elimination of mis-folded proteins. The proteasome content and activity in patient's fibroblasts was however unaffected. Nevertheless, patient's cells exhibited impaired protein homeostasis characterized by accumulation of ubiquitinated proteins suggesting severe proteotoxic stress. Indeed, the TCF11/Nrf1 transcriptional pathway allowing proteasome recovery after proteasome inhibition is permanently activated in the patient's fibroblasts. Upon chemical proteasome inhibition this pathway was impaired in patient's cells, which were unable to compensate for proteotoxic stress although a higher proteasome content and activity was observed. PSMC3 knock-out in zebrafish remarkably reproduced the human phenotype with inner ear development anomalies as well as cataracts suggesting that Rpt5 plays a major role in inner ear, lens and central nervous system development. This report is the first description of a recessive pathogenic variant in a proteasomal ATPase. $19 \mathrm{~S}$ subunits are highly expressed in the central nervous system and appear to be of utmost importance for neuronal /sensorial protein homeostasis and development.

A. Kröll-Hermi: None. F. Ebstein: None. C. Stoetzel: None. V. Geoffroy: None. E. Schaefer: None. S. Scheidecker: None. S. Bär: None. M. Takamiya: None. K. Kawakami: None. B. Zieba: None. F. Studer: None. V. Pelletier: None. C. Speeg-Schatz: None. V. Laugel: None. D. Lipsker: None. F. Sandron: None. S. McGinn: None. A. Boland: None. J. Deleuze: None. L. Kuhn: None. J. Chicher: None. P. Hammann: None. S. Friant: None. C. 
Etard: None. E. Krüger: None. J. Muller: None. U. Strähle: None. H. Dollfus: None.

\section{C07 Impact of Genomic Medicine on Public and Patients}

\section{C07.1}

Implementing a hybrid clinical/research model in genomic medicine: post 100,000 Genomes Project

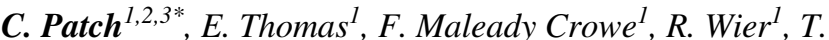
Fowler $^{1}$, M. Bishop ${ }^{4}$, A. Pichini ${ }^{4}$

${ }^{1}$ Genomics England, London, United Kingdom, ${ }^{2}$ Wellcome Genome Campus, Cambridge, United Kingdom, ${ }^{3}$ Sheffield Hallam University, Sheffield, United Kingdom, ${ }^{4}$ Genomics Education Programme, Health Education England, Birmingham, United Kingdom

Building on the UK 100,000 Genomes Project and existing NHS genomic infrastructure, the creation of the NHS Genomics Medicine service by NHS England together with Genomics England has established the principle of patient choice to both have a diagnostic test and agree to use of their data for the research. Through the national test directory whole genome sequencing (WGS) has been commissioned for clinical diagnostic use for 21 rare disease clinical indications and 4 cancer indications (https://www.england. nhs.uk/publication/national-genomic-test-directories/). All persons agreeing to the WGS testing will be offered the opportunity to agree to the use of their data and available samples within the National Genomic Research Library. This will be part of the clinical pathway. The development of the model and the development of the necessary complex implementation processes was underpinned by collaboration and informal co-design. This included ensuring the choice model was compliant with current understandings of governance across the clinical and research interface, development of the Genomics England research environment, development of materials to support patients and the clinical workforce and formalising processes for clinical implementation. Implementation was informed by a series of multi-agency task and finish groups and by consultation with health care professionals, educationalists, participants of the 100,000 genomes project and other stakeholders. Here we present the finalised products for the first stage of the Genomics Medicine Service. The long-term aim being to create an ethically robust and trusted research ecosystem as a routine part of clinical care.

C. Patch: A. Employment (full or part-time); Significant; Genomics England. E. Thomas: A. Employment (full or part-time); Significant; Genomics England. F. Maleady Crowe: A. Employment (full or part-time); Significant;
Genomics England. R. Wier: A. Employment (full or parttime); Significant; Genomics England. T. Fowler: A. Employment (full or part-time); Significant; Genomics England. M. Bishop: None. A. Pichini: None.

\section{C07.2}

A tailored approach towards informing relatives at risk of inherited cardiac diseases: preliminary results of a randomized controlled trial

L. M. Van den Heuvel ${ }^{*}$, Y. M. Hoedemaekers ${ }^{2}$, A. F. Baas $^{3}$, M. J. H. Baars ${ }^{l}$, E. M. A. Smets ${ }^{\text {I }}$ J. P. van Tintelen ${ }^{3}$, I. Christiaans ${ }^{4}$

${ }^{1}$ Amsterdam UMC, location AMC, Amsterdam, Netherlands, ${ }^{2}$ Radboud University Medical Centre, Nijmegen, Netherlands, ${ }^{3}$ University Medical Centre Utrecht, Utrecht, Netherlands, ${ }^{4}$ University Medical Centre Groningen, Groningen, Netherlands

Introduction: If undetected, inherited cardiac diseases can lead to sudden cardiac death, while preventive and treatment options are available. Predictive DNA testing is advised for at-risk relatives. Currently, probands are asked to inform relatives with a family letter. However, only half of relatives attends genetic counselling.

Methods: Current practice was compared to a tailored approach, in which probands were asked to decide to (initially) inform relatives themselves or by the counsellor, and family letters were sent directly 1 month after disclosure of the probands' test result with consent of the proband. Outcomes were uptake of counselling in the first year (using pedigrees), and impact on psychological/family functioning, and evaluation of the approach (measured with surveys at 3 (T1) and 9 (T2) months after disclosure).

Results: 96 probands were included; 549 relatives were eligible for counselling. Preliminary findings showed no significant difference in uptake of genetic counselling between the control (34.5\%) and the intervention (36.7\%) group. At T1, 92\% of probands in the intervention group reported that all relatives were informed, compared to $73 \%$ in the control group. In both the intervention and control group, probands were satisfied with the approach (resp. $95 \%$ vs. $88 \%$ ) and reported no impact on family functioning (resp. $94.6 \%$ vs. $92.3 \%$ ).

Conclusions: Preliminary findings of this trial show no differences in uptake, impact on family functioning and satisfaction between the tailored and current approach, despite more relatives being informed using the tailored approach. Data collection of uptake and T2 surveys will be finished in November 2020. 
L. M. Van den Heuvel: None. Y. M. Hoedemaekers: None. A. F. Baas: None. M. J. H. Baars: None. E. M. A. Smets: None. J. P. van Tintelen: None. I. Christiaans: None.

\section{C07.3}

Measuring the burden of direct-to-consumer genetic testing on clinical genetics services

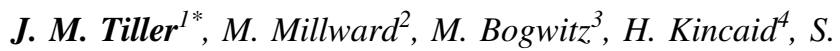
Taylor $^{3}$, A. Trainer ${ }^{3,2}$, P. Lacaze ${ }^{l}$

${ }^{1}$ Public Health Genomics, Monash University, Melbourne, Australia, ${ }^{2}$ University of Melbourne, Melbourne, Australia, ${ }^{3}$ Genomic Medicine \& Parkville Familial Cancer Centre, Melbourne, Australia, ${ }^{4}$ Monash Health, Melbourne, Australia

Introduction: Direct-to-consumer genetic testing (DTCGT) is increasing in popularity, as is the practice of consumers interrogating their resultant genotype data with third-party software tools to generate unvalidated imputed disease risk estimates. This is suspected to be creating a burden on clinical genetic health services, as consumers seek assistance with interpretation. However, few studies have collected recent evidence regarding the extent of this burden.

Methods and results: We administered an online survey to Australian clinical genetic services, asking questions related to DTCGT-related referrals over the past 10 years. Eleven publicly-funded clinical genetic services completed the survey, reporting over 100 DTCGT-related referrals. Most referrals (83\%) were made by general practitioners to aid interpretation of DTCGT results. Over 30\% of referrals were related to imputed disease risk estimates generated from third-party web-based software tools. Services reported low validation rates for DTCGT results $(<10 \%)$. Procedures for managing DTCGT referrals and granting appointments were variable between services, with most services (8/11) lacking specific procedures.

Conclusions: Our study provides evidence that the increasing popularity of DTCGT is causing a material burden on publicly-funded Australian clinical genetics services. We also report a sizeable portion of that burden resulting from the use of third-party genetic risk-imputation software tools. The impact of DTCGT is likely to increase in coming years, if the use of DTCGT continues to grow. Our findings are relevant for other countries with publiclyfunded or single-payer clinical genetics services, which will have to contend with the growing popularity of DTCGT in the near future.

J. M. Tiller: None. M. Millward: None. M. Bogwitz: None. H. Kincaid: None. S. Taylor: None. A. Trainer: None. P. Lacaze: None.

\section{C07.4}

Critical components of informed consent for genetic testing: Results of a Delphi Consensus process

K. E. Ormond ${ }^{1 *}$, M. Borensztein ${ }^{l}$, A. H. Buchanan ${ }^{2}, W$. Faucett $^{2}$, M. L. G. Hallquist ${ }^{2}$, H. L. Peay ${ }^{3}$, M. E. Smith ${ }^{4}$, E.

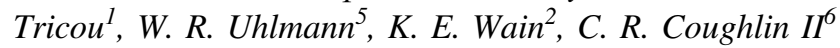

${ }^{1}$ Stanford University, Stanford, CA, United States, ${ }^{2}$ Geisinger Health Care, Danville, PA, United States, ${ }^{3}$ RTI, Research Triangle Park, NC, United States, ${ }^{4}$ Northwestern University, Chciago, IL, United States, ${ }^{5}$ University of Michigan, Ann Arbor, MI, United States, ${ }^{6}$ University of Colorado, Denver, CO, United States

Background: Genetic testing has historically been tied to pre-test genetic counseling to facilitate informed consent. While core concepts exist for general informed consent, there is not specific guidance defining them around the clinical use of genetic testing.

Method: We conducted a scoping literature review that identified 77 concepts, and used a modified Delphi methodology to survey experts. Participants used 5 point likert scales to determine how "critical" each concept was for a "typical patient" to make an informed decision, and ranked the top choices within each subcategory of concepts over 2 rounds.

Findings: There were 25 respondents $(9$ medical geneticists, 8 genetic counselors, 9 bioethicists), $60 \%$ of whom regularly consent for genetic testing. The most highly endorsed concepts for inclusion were: (1) condition(s) tested; (2) management implications; (3) primary and (4) secondary results to be returned, and options for choice; (5) To whom results will be reported; (6) voluntariness of testing; (7) test limitations (8) potential to learn unexpected information about family relationships; (9) potential impact on personal health, (10) on treatment/screening, and (11) on the family (health, emotions, relationships); (12) risks for discrimination and/or stigma. A larger survey, meant to validate whether medical geneticists and genetic counselors more broadly agree with the Delphi results, will be conducted in spring 2020 and included in the presentation.

Discussion: Defining the minimal critical concepts to allow clients to reach informed decisions about genetic testing is important as genetic testing increases exponentially and the consent process is increasingly done by nongenetics healthcare providers.

K. E. Ormond: None. M. Borensztein: None. A. H. Buchanan: None. W. Faucett: None. M. L. G. Hallquist: None. H. L. Peay: None. M. E. Smith: None. E. Tricou: None. W. R. Uhlmann: None. K. E. Wain: None. C. R. Coughlin II: None. 


\section{C07.5}

Public attitudes towards human germline gene editing: a baseline survey in the Netherlands

D. Houtman ${ }^{1 *}$, M. Polak ${ }^{2}$, B. Vijlbrief ${ }^{1}$, P. Verheggen ${ }^{3}, R$. Hofstra $^{l}$, S. Riedijk ${ }^{l}$

${ }^{1}$ Erasmus Medical Center, Rotterdam, Netherlands, ${ }^{2}$ Erasmus University Rotterdam, Rotterdam, Netherlands, ${ }^{3}$ Motivaction International B. V., Rotterdam, Netherlands

Introduction: A multidisciplinary consortium is organizing a public dialogue about human germline gene editing (GLGE) in the Netherlands. Challenges of public dialogue include ensuring inclusivity, designing appropriate content for engagement, and evaluating the impact of the dialogue on opinion formation. The current study provides a baseline measurement, which will later be used to evaluate the impact of the dialogue. For now, we aim to use this measurement to identify subgroups of society based on their attitudes towards human GLGE and their willingness to engage in science and technology.

Materials and Methods: A representative sample of 1185 adults filled out an online survey, measuring their acceptance of different applications of human GLGE and their willingness to discuss new technologies and how to deal with them.

Results: By means of exploratory analyses we found that in our sample $68.9 \%$ agreed with application to prevent a severe muscle disease, $37.0 \%$ agreed with application to prevent an infection disease, and $7.8 \%$ agreed with application to increase intelligence. Willingness to discuss new technologies and how to deal with them was associated with income $\left(\chi^{2}(3)=15.577, p<0.01\right)$, educational level $\left(\chi^{2}(2)=25.830, p<0.01\right)$, and personal values $\left(\chi^{2}(7)=\right.$ $68.930, p<0.01)$. Comparatively, people who did not want to discuss new technologies and how to deal with them (36.5\%) showed lower educational-level, lower income, and more traditional and consumption-oriented values.

Conclusions: Our data provide insights into attitudes of the Dutch population towards different applications of human GLGE and into the identification of subgroups of society for whom participation is not self-evident.

D. Houtman: None. M. Polak: None. B. Vijlbrief: None. P. Verheggen: A. Employment (full or part-time); Modest; Motivaction International B. V. R. Hofstra: None. S. Riedijk: None.

\section{C07.6}

Does Genetic Counseling lead to effective coping with genetic risk information?

B. B. Biesecker ${ }^{*}, M . T . C h o^{2}$
${ }^{1}$ RTI, International, Bethesda, MD, United States, ${ }^{2}$ NHGRI, National Institutes of Health, Bethesda, MD, United States

Patient coping has been intermittently studied as a practice outcome of genetics services. Yet, assessments have been inconsistent and coping strategies have been reported without evidence of their effectiveness. Discrepant theoretical and methodological approaches may have distracted attention from compelling evidence that genetic counseling can enhance effective patient coping and, with time, adaptation to genetic information. We report key findings from a systematic literature review of 68 abstracts that met our inclusion criteria for studies of coping with genetic risk or a condition. The studies represent $\mathbf{1 2 , 0 0 6}$ patients. Study designs included: cross-sectional (31), longitudinal (10), randomized (5), and observational (1). Among qualitative studies were semi-structured interviews (16) and focus groups (3). The majority were studies conducted in cancer genetics, yet most specialty settings were represented. A synthesis of outcomes generated five hypotheses: H1: The effectiveness of coping strategies depends on their congruence with the stress source and its actionability. $\mathrm{H} 2$ : In the face of genetic risk, avoidant coping is most likely to result in psychological distress, unless used to avoid learning unwanted genetic information. H3: Family history, risk perception and personality traits mediate effective use of coping strategies to manage illness-related stress. H4: Identifying unhelpful coping strategies and offering useful ones reduces risk-related distress and promotes adaptation. H5: Enhancing patient coping self-efficacy improves coping effectiveness. Overall, the evidence that generated these hypotheses suggests that genetics providers have important clinical opportunities to enhance coping effectiveness as a valuable outcome in helping patients adapt to genetic risk or a condition.

B. B. Biesecker: None. M. T. Cho: None.

\section{C08 Impact of Diagnostics Improvements in Healthcare}

\section{C08.1}

Project Baby Bear: The first state-funded quality improvement project of rapid Whole Genome Sequencing in neonatal and pediatric intensive care units in the USA
K. A. Ellsworth ${ }^{1 *}$, S. Caylor ${ }^{1}$, W. Benson ${ }^{l}$, C. Ashburner ${ }^{2}$, J. Carmichael ${ }^{2}$, E. Cham ${ }^{3}$, S. Chowdhury ${ }^{I}, J$. Cleary ${ }^{4}$, A. $D^{\prime}$ Harlingue $^{3}$, L. Farnaes ${ }^{l}, J$. Hunt ${ }^{4}$, C. Hobbs ${ }^{l}, K$. Houtchens $^{3}$, P. Joe ${ }^{3}$, J. Knight ${ }^{4}$, A. Kochhar ${ }^{2}$, M. Joseph ${ }^{2}$, J. Limon $^{2}$, M. Martin ${ }^{5}$, S. Nahas ${ }^{1}$, K. A. Rauen ${ }^{5}$, A. Schwarz ${ }^{4}$, S. P. Shankar ${ }^{5}$, R. Spicer ${ }^{2}$, M. Rojas ${ }^{2}$, O. Vargas-Shiraishi ${ }^{4}$, K. Wigby ${ }^{l}$, N. Zadeh ${ }^{4}$, S. Kingsmore ${ }^{l}$, D. Dimmock ${ }^{l}$ 
${ }^{1}$ Rady Children's Institute for Genomic Medicine, San Diego, CA, United States, ${ }^{2}$ Valley Children's Healthcare, Madera, CA, United States, ${ }^{3}$ University of California - San Francisco, Benioff Children's Hospital Oakland, Oakland, CA, United States, ${ }^{4}$ Children's Hospital of Orange County, Orange, CA, United States, ${ }^{5}$ University of California Davis Children's Hospital, Sacramento, CA, United States

Previous studies showed that rapid Whole Genome Sequencing (rWGS) results in improved outcomes and reduced costs for acutely ill infants in the intensive care unit (ICU) when compared to standard of care. Currently, rWGS is not a reimbursed test under California Medicaid (Medi$\mathrm{Cal}$ ), private insurers or federal Medicare programs. California's State Department of Health Care Services appropriated \$2,000,000 for rWGS Quality Improvement Pilot Program, known as Project Baby Bear (PBB) with a goal to identify the clinical and economic benefits of rWGS for critically ill pediatric patients enrolled in Medi-Cal. rWGS was performed on 116 babies admitted to five medical centers in California. Data was collected on the outcomes of patients and costs to evaluate the clinical and economic benefits of rWGS. The clinical utility of diagnoses and changes in outcome were determined by patients' medical teams. To date, $43 \%$ of those sequenced received a molecular diagnosis, with turnaround time for provisional results in $<72$ hrs. In $>35 \%$ of cases, rWGS altered the clinical management. Collectively, patients avoided $>225$ hospital days, numerous invasive procedures and diagnostic tests. In the preliminary analysis of PBB cases, it was estimated a charge expenditure saving of up to $\$ 2.4 \mathrm{M}$ across all episodes of care. In conclusion, the preliminary data from PBB demonstrated the clinical utility and cost effectiveness of utilization of rWGS in the pediatric ICU setting. PBB provided evidence that rWGS should become a Medi-Cal covered benefit, and presented the framework for future studies in other states to show the utility of rWGS.

K. A. Ellsworth: None. S. Caylor: None. W. Benson: None. C. Ashburner: None. J. Carmichael: None. E. Cham: None. S. Chowdhury: None. J. Cleary: None. A. D'Harlingue: None. L. Farnaes: None. J. Hunt: None. C. Hobbs: None. K. Houtchens: None. P. Joe: None. J. Knight: None. A. Kochhar: None. M. Joseph: None. J. Limon: None. M. Martin: None. S. Nahas: None. K. A. Rauen: None. A. Schwarz: None. S. P. Shankar: None. R. Spicer: None. M. Rojas: None. O. Vargas-Shiraishi: None. K. Wigby: None. N. Zadeh: None. S. Kingsmore: None. D. Dimmock: None.

\section{C08.2}

Integration of genome sequencing into health care experiences from 3211 rare disease patients show high diagnostic rates across multiple clinical entities
A. Lindstrand ${ }^{1,2 *}$, H. Stranneheim ${ }^{1,3,4}$, K. Lagerstedt-

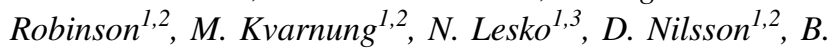
Anderlid $^{1,2}$, H. Arnell ${ }^{5}$, C. Backman Johansson ${ }^{3}, M$. Barbaro $^{3}$, E. Björck ${ }^{1,2}, H$. Bruhn ${ }^{3}$, J. Eisfeldt ${ }^{1,2,4}, M$. Engvall $^{3}$, C. Freyer ${ }^{3}$, G. Grigelioniene ${ }^{1,2}$, P. Gustavsson ${ }^{1,2}$, A. Hammarsjö ${ }^{1,2}$, M. Hellström-Pigg ${ }^{1,2}$, A. Jemt $^{6}, M$. Laaksonen $^{7}$, S. Lind Enoksson ${ }^{8}$, M. Magnusson ${ }^{1,7}, \mathrm{H}$. Malmgren $^{1,2}$, K. Naess $^{3}$, M. Nordenskjöld ${ }^{1,2}$, M. Oscarson ${ }^{3}$, M. Pettersson ${ }^{1,2}$, C. Rasi ${ }^{4}$, A. Rosenbaum ${ }^{7}$, E. Sahlin ${ }^{1,2}, T$. Stödberg ${ }^{3,5}$, B. Tesi $^{1,2}$, E. Tham ${ }^{1,2}$, H. Thonberg ${ }^{1,2}$, U. von Döbeln $^{3}, S$. Vonlanthen ${ }^{8}$, A. Wikström ${ }^{8}, J$. Wincent ${ }^{1,2}, O$. Winqvist $^{8}$, A. Wredenberg ${ }^{3}$, S. Ygberg ${ }^{3,5}$, R. H. Zetterström ${ }^{3}$, P. Marits ${ }^{8}$, M. Johansson-Soller ${ }^{1}$, M. Johansson Soller ${ }^{1,2}$, A. Nordgren ${ }^{1,2}$, V. Wirta ${ }^{4,6,7}$, A. Wedell ${ }^{1,3}$

${ }^{1}$ Department of Molecular Medicine and Surgery, Karolinska Institutet, Stockholm, Sweden, ${ }^{2}$ Department of Clinical Genetics, Karolinska University Hospital, Stockholm, Sweden, ${ }^{3}$ Centre for Inherited Metabolic Diseases, Karolinska University Hospital, Stockholm, Sweden, ${ }^{4}$ Science for Life Laboratory, Department of Microbiology, Tumour and Cell Biology, Karolinska Institutet, Stockholm, Sweden, ${ }^{5}$ Department of Women's and Children's Health, Karolinska Institutet, Stockholm, Sweden, ${ }^{6}$ Genomic Medicine Center Karolinska, Karolinska University Hospital, Stockholm, Sweden, ${ }^{7}$ Science for Life Laboratory, School of engineering sciences in Chemistry, Biotechnology and Health, KTH Royal Institutet of Technology, Stockholm, Sweden, ${ }^{8}$ Department of Clinical Immunology and Transfusion Medicine, Karolinska University Hospital, Stockholm, Sweden., Stockholm, Sweden

We report the findings from 4553 samples (3211 cases) that have been analyzed by genome sequencing at the Genomic Medicine Center Karolinska-Rare Diseases (GMCK-RD). GMCK-RD represents a long-term collaborative initiative between Karolinska University Hospital and SciLifeLab to establish advanced, genomics-based diagnostics in the Stockholm healthcare setting. Our bioinformatic analysis pipeline includes SNVs, Indels, CNVs (including SMN), balanced structural variants and short tandem repeat expansions. Visualization of results for clinical interpretation is carried out in Scout - a custom-developed decision support system. Both singleton (84\%) and trio/family (16\%) analysis is performed. The variant interpretation is done by 15 expert teams at the hospital involving staff from three clinics and for patients with complex phenotypes data is shared between the teams. Overall $33 \%$ of the patients receive a molecular diagnosis (1208 positive findings) ranging from $30 \%$ to $60 \%$ for specific disease groups. There is extreme heterogeneity regarding causative genes $(n=701)$ with some of the most common being $\operatorname{SCNIA~(~} n=13$; epilepsy), TNFRSF13B ( $n=5$; inborn errors of immunity) and 
ARIDIB ( $n=8$; intellectual disability). Some of the causative variants were recurrent; including both previously known founder mutations, known recurrent de novo mutations and novel recurrent variants. Overall, GMCK-RD has had dramatic results as a large number of patients have received specific molecular diagnoses. Furthermore, negative cases are systematically included in research studies that have led to the discovery of 21 novel disease-causing genes. To facilitate discovery of new disease genes, GMCK-RD has joined international data sharing initiatives, including ClinVar, UDNI, Beacon and MatchMaker Exchange.

A. Lindstrand: None. H. Stranneheim: None. K. Lagerstedt-Robinson: None. M. Kvarnung: None. N. Lesko: None. D. Nilsson: None. B. Anderlid: None. H. Arnell: None. C. Backman Johansson: None. M. Barbaro: None. E. Björck: None. H. Bruhn: None. J. Eisfeldt: None. M. Engvall: None. C. Freyer: None. G. Grigelioniene: None. P. Gustavsson: None. A. Hammarsjö: None. M. HellströmPigg: None. A. Jemt: None. M. Laaksonen: None. S. Lind Enoksson: None. M. Magnusson: None. H. Malmgren: None. K. Naess: None. M. Nordenskjöld: None. M. Oscarson: None. M. Pettersson: None. C. Rasi: None. A. Rosenbaum: None. E. Sahlin: None. T. Stödberg: None. B. Tesi: None. E. Tham: None. H. Thonberg: None. U. von Döbeln: None. S. Vonlanthen: None. A. Wikström: None. J. Wincent: None. O. Winqvist: None. A. Wredenberg: None. S. Ygberg: None. R. H. Zetterström: None. P. Marits: None. M. Johansson-Soller: None. M. Johansson Soller: None. A. Nordgren: None. V. Wirta: None. A. Wedell: None.

\section{C08.3}

Genomic loci susceptible to systematic sequencing bias in clinical whole genomes

\section{T. M. Freeman ${ }^{1 *}$, Genomics England Research Consortium, D. Wang ${ }^{1}$, J. Harris ${ }^{2}$}

${ }^{I}$ The University of Sheffield, Sheffield, United Kingdom, ${ }^{2}$ Personalis Inc., Menlo Park, CA, United States

Introduction: Accurate massively parallel sequencing (MPS) of genetic variants is key to many areas of science and medicine, including diagnosing genetic diseases. Certain genomic positions can be prone to higher rates of systematic sequencing and alignment bias that limit accuracy, resulting in false positive variant calls. Current standard practices to differentiate between loci that can and cannot be sequenced with high confidence utilise consensus between different sequencing methods as a proxy for sequencing confidence. These practices have significant limitations and alternative methods are required to overcome these.
Materials and Methods: We have developed a novel statistical method based on summarising sequenced reads from whole genome clinical samples and cataloguing them in "Incremental Databases" that maintain individual confidentiality. Allele statistics were catalogued for each genomic position that consistently showed systematic biases with the corresponding MPS pipeline.

Results: We found systematic biases present at $\sim 1-3 \%$ of the human autosomal genome across five patient cohorts. We identified how susceptible different genomic regions were to systematic biases, including large homopolymer flanks (odds ratio $=23.29-33.69$ ) and the NIST high confidence genomic regions (odds ratio $=0.154-0.191$ ). We confirmed our predictions on a gold-standard reference genome and showed that these systematic biases can lead to suspect variant calls within clinical panels.

Conclusion: Our results recommend increased caution to address systematic sequencing biases. This study implements a novel statistical approach to enhance quality control of clinically sequenced samples by flagging variants at suspect loci for further analysis or exclusion.

This work has recently been accepted for publication in Genome Research.

T. M. Freeman: None. D. Wang: None. J. Harris: A. Employment (full or part-time); Significant; Personalis Inc. E. Ownership Interest (stock, stock options, patent or other intellectual property); Significant; Personalis Inc.

\section{C08.4}

Systematic analysis of short tandem repeats in $\mathbf{3 8 , 2 5 6}$ exomes provides additional diagnostic yield

\section{B. P. G. H. van der Sanden ${ }^{1,2 *}, M$. de Groot ${ }^{l}, J$. Corominas $^{1}$, M. Pennings ${ }^{1}$, R. P. P. Meijer ${ }^{1}$, L. E. L. M. Vissers $^{1,2}$, E. Kamsteeg ${ }^{1}$, C. Gilissen ${ }^{1,3}$ \\ ${ }^{1}$ Department of Human Genetics, Radboudumc, Nijmegen, Netherlands, ${ }^{2}$ Donders Institute for Brain, Cognition and Behaviour, Nijmegen, Netherlands, ${ }^{3}$ Radboud Institute for Molecular Life Sciences, Nijmegen, Netherlands}

Short tandem repeats (STRs) are defined as tandemly repeated nucleotide motifs of 2-12 base pairs in length and occur across the genome. Extreme expansion of specific STRs can lead to approximately 30 different human genetic disorders. Despite the extensive application of whole exome sequencing (WES) in routine diagnostic genetic testing, STRs are not routinely identified from these data. Here, we present our results of a systematic analysis for STR expansion disorders in our cohort of 38,256 clinical exomes.

We evaluated four different tools, exSTRa, STRetch, ExpansionHunter, and GangSTR, on a validation cohort of 
11 patients with known pathogenic repeat expansion allele (s). ExpansionHunter demonstrated highest sensitivity $(91 \%)$ and specificity $(100 \%)$, after which we applied it to 2,900 exomes from movement and muscle disorder patients.

We identified 49 patients with a likely aberrant STR length. Validation by GeneScan ${ }^{\circledR}$ and/or triplet repeatprimed PCR technologies confirmed the presence of aberrant expansion allele(s) for $18(0.6 \%)$. Importantly, 10 STR expansions had not previously been identified using conventional genetic testing, and may result in novel diagnoses. Three expansions were detected in ATXN7, known to cause spinocerebellar ataxia type 7 , for which increase in repeat size between generations may cause extreme anticipation. We subsequently tested the remainder of our diagnostic WES cohort, including over 30 clinically and genetically heterogeneous disorders, and currently 1,187 aberrant expansion alleles are being validated.

Our results show that systematic STR evaluation may increase diagnostic WES yield by $0.6 \%$, and should become a routine part of WES data analysis in genetic testing laboratories.

B. P. G. H. van der Sanden: None. M. de Groot: None. J. Corominas: None. M. Pennings: None. R. P. P. Meijer: None. L. E. L. M. Vissers: None. E. Kamsteeg: None. C. Gilissen: None.

\section{C08.5}

A cost-effectiveness and utility analysis of genomic sequencing in a prospective versus historical cohort of complex pediatric patients

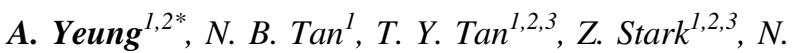
Brown $^{1,3}$, M. J. Hunter ${ }^{4,5}$, M. Delatycki ${ }^{1,3}$, C. Stutterd ${ }^{1,3}, R$. Savarirayan $^{1,3}$, G. McGillivray ${ }^{l}$, R. Stapleton ${ }^{l}$, S. Kumble ${ }^{l}$, L. Downie ${ }^{1}$, M. Regan ${ }^{4,5}$, S. Lunke ${ }^{1}$, B. Chong ${ }^{1}$, D. Phelan ${ }^{1}$, G. Brett ${ }^{1}$, A. Jarmolowicz', Y. Prawer ${ }^{4}, G$. Valente ${ }^{6}, Y$.

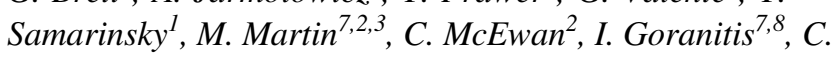
Gaff $^{7,2,3}$, S. M. White W,2,3 $^{1,3}$

${ }^{1}$ Victorian Clinical Genetics Services, Murdoch Children's Research Institute, Parkville, Australia, ${ }^{2}$ Melbourne Genomics Health Alliance, Parkville, Australia, ${ }^{3}$ Department of Paediatrics, University of Melbourne, Parkville, Australia, ${ }^{4}$ Monash Genetics, Monash Health, Melbourne, Australia, ${ }^{5}$ Department of Paediatrics, Monash University, Melbourne, Australia, ${ }^{6}$ Genetics in the North East, Austin Health, Melbourne, Australia, ${ }^{7}$ Murdoch Children's Research Institute, Parkville, Australia, ${ }^{8}$ Centre for Health Policy, Melbourne School of Population and Global Health, University of Melbourne, Parkville, Australia

The diagnosis of children with genetic conditions places a significant economic burden on health care services. The lack of well-defined comparative cohorts has been a limitation of health economic studies comparing first-line genomic sequencing (GS) against traditional approaches.

Aim: To evaluate utility and cost effectiveness of early GS in pediatric patients with complex monogenic conditions compared to a matched historical cohort.

Method: Data, including diagnosis rate and investigation costs, were collected in a prospective cohort of 92 pediatric patients who underwent singleton GS over an 18-month period (2016-2017). Inclusion required patients to have two of the following: a condition with high morbidity or mortality, a multi-system disease involving three or more organ systems, or severe limitation of daily function. For comparison, data were collected in a historical patient cohort fulfilling the inclusion criteria who underwent traditional investigations in the two years (2012-2013) prior to the availability of clinical genomic sequencing.

Results: Genomic sequencing yielded a diagnosis in $42 \%$ while traditional investigations yielded a diagnosis in $23 \%$ $(\mathrm{p}=0.003$ ). $75 \%$ of diagnosed patients experienced a change in management, compared to $33 \%$ of diagnosed patients who underwent traditional investigations. In the GS cohort, four times fewer invasive investigations were observed. Compared to traditional investigations, singleton genomic sequencing at a cost of $\$ 3100$ AUD resulted in a mean saving per person of $\$ 2780$ AUD (95\%CI $\$ 1585$ \$3974).

Conclusion: Early genomic sequencing is highly costeffective while doubling the diagnostic yield of traditional approaches and improving the care of patients with complex genetic conditions.

A. Yeung: None. N. B. Tan: None. T. Y. Tan: None. Z. Stark: None. N. Brown: None. M. J. Hunter: None. M. Delatycki: None. C. Stutterd: None. R. Savarirayan: None. G. McGillivray: None. R. Stapleton: None. S. Kumble: None. L. Downie: None. M. Regan: None. S. Lunke: None. B. Chong: None. D. Phelan: None. G. Brett: None. A. Jarmolowicz: None. Y. Prawer: None. G. Valente: None. Y. Samarinsky: None. M. Martin: None. C. McEwan: None. I. Goranitis: None. C. Gaff: None. S. M. White: None.

\section{C08.6}

The impact of the 100,000 Genomes Project on rare disease in national healthcare

D. Smedley ${ }^{1,2^{*}}$, S. Abbs ${ }^{3}$, G. Arno ${ }^{4}$, E. Baple ${ }^{5}$, M. Barnes ${ }^{1}$, P. Beales ${ }^{6}$, M. Bitner-Glindzicz ${ }^{6}$, G. Black ${ }^{7}$, P. Brennan ${ }^{8}, G$.

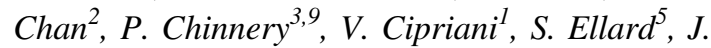
Ellingford $^{7}$, P. Elliott ${ }^{6}, H$. Firth ${ }^{3}$, F. Flintner ${ }^{10}$, K. Ibanez Garikano $^{1,2}$, H. Houlden ${ }^{6}$, M. Irving ${ }^{10}$, J. Jacobsen ${ }^{1}$, E. McDonagh $^{2}$, D. McMullan ${ }^{11}$, L. Moutsianas ${ }^{1,2}$, W. Newman ${ }^{7}$, W. H. Ouwehand ${ }^{3,9,12}$, T. Ratnaike ${ }^{3}$, A. Rueda

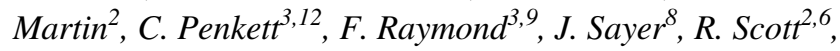




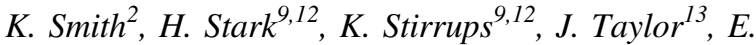
Thomas $^{2}$, A. Tucci ${ }^{2,1,6}, J^{2}$ Vandrovcova $^{6}$, L. Vestito $^{6}$, A. Webster $^{4}$, W. Wei ${ }^{9}$, M. Wielscher ${ }^{2}, H$. Williams ${ }^{6}, A$. Wilkie $^{13}$, C. Wright ${ }^{5}$, A. Rendon ${ }^{2,9}$, M. Caulfield $^{2,1}$, . NIHR BioResource $^{3}$, . Genomics England Research Consortium ${ }^{2}$

${ }^{1}$ Queen Mary University of London, London, United Kingdom, ${ }^{2}$ Genomics England, London, United Kingdom, ${ }^{3}$ Cambridge University Hospitals, Cambridge, United Kingdom, ${ }^{4}$ Moorfields Eye Hospital, London, United Kingdom, ${ }^{5}$ University of Exeter, Exeter, United Kingdom, ${ }^{6}$ University College London, London, United Kingdom, ${ }^{7}$ University of Manchester, Manchester, United Kingdom, ${ }^{8}$ The Newcastle upon Tyne NHS Foundation Trust, Newcastle, United Kingdom, ${ }^{9}$ University of Cambridge, Cambridge, United Kingdom, ${ }^{10}$ Guys and St Thomas' NHS Foundation Trust, London, United Kingdom, ${ }^{11}$ Birmingham Women's and Children's NHS Trust, Birmingham, United Kingdom, ${ }^{12} \mathrm{NHS}$ Blood and Transplant, Cambridge, United Kingdom, ${ }^{13}$ University of Oxford, Oxford, United Kingdom

Introduction: The UK 100,000 Genomes Project was created to define the role of whole genome sequencing (WGS) in rare disease, cancer and infection. For rare disease a diagnostic pipeline and research environment was built to address the needs of patients who remained undiagnosed following standard testing in the National Health Service (NHS) in England.

Methods: Detailed clinical features on enrolled participants were captured, WGS performed and automated variant interpretation run based on gene panels (PanelApp) as well as phenotype-based prioritisation (Exomiser). Prioritised variants were returned to the NHS for laboratory review and clinical reporting.

Results: We report on the first 4660 participants across a broad spectrum of rare disease. Diagnostic yields varied by family structure (highest in trios and larger pedigrees) and disease. Likely Mendelian disorders had higher yields (37\%) with intellectual disability, hearing and eye disorders achieving 40-50\%. Those with more complex causes (e.g. familial cancer) had lower yields $(11 \%)$, particularly where known loci had been excluded by standard tests. The overall diagnostic yield was $26 \%$, involving 470 unique diagnoses. Clinical phenotypes (Human Phenotype Ontology terms) were critical to performance of the automated pipeline in detecting $90 \%$ of SNV/indel diagnoses. WGS enabled diagnoses that would be missed by other technologies: $4 \%$ involved non-coding variation, $9 \%$ with structural variants, and $8 \%$ in coding regions with no/low coverage by whole exome sequencing. Finally, cohort-wide analysis detected numerous novel disease-gene associations.

Conclusions: Our findings will inform healthcare uptake and utilisation of WGS world-wide, identifying and enabling prioritization of clinical indications for adoption.

D. Smedley: F. Consultant/Advisory Board; Modest; Congenica Ltd. S. Abbs: None. G. Arno: None. E. Baple: None. M. Barnes: None. P. Beales: None. M. BitnerGlindzicz: None. G. Black: None. P. Brennan: None. G. Chan: None. P. Chinnery: None. V. Cipriani: None. S. Ellard: None. J. Ellingford: None. P. Elliott: None. H. Firth: None. F. Flintner: None. K. Ibanez Garikano: None. H. Houlden: None. M. Irving: None. J. Jacobsen: None. E. McDonagh: None. D. McMullan: None. L. Moutsianas: None. W. Newman: None. W. H. Ouwehand: None. T. Ratnaike: None. A. Rueda Martin: None. C. Penkett: None. F. Raymond: None. J. Sayer: None. R. Scott: None. K. Smith: None. H. Stark: None. K. Stirrups: None. J. Taylor: None. E. Thomas: None. A. Tucci: None. J. Vandrovcova: None. L. Vestito: None. A. Webster: None. W. Wei: None. M. Wielscher: None. H. Williams: None. A. Wilkie: None. C. Wright: None. A. Rendon: None. M. Caulfield: None. NIHR BioResource: None. Genomics England Research Consortium: None.

\section{C09 Metabolic and Mitochondrial Disorders}

\section{C09.1}

The Australian Genomic Health Alliance Mitochondrial Flagship - A national approach to genomic diagnostics

D. Thorburn ${ }^{1,2,3^{*}}$, N. Baker ${ }^{3}$, S. Balasubramaniam ${ }^{4}, D$.

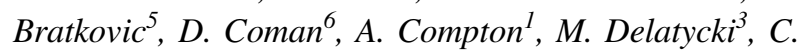

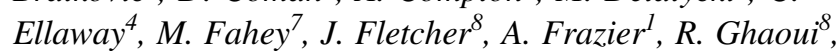
H. Goel ${ }^{9}$, D. Hock ${ }^{10}$, M. Kava ${ }^{11}$, N. Lake ${ }^{12}$, P. Lamont ${ }^{13}$, J.

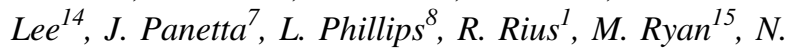

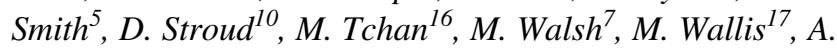
Welch $^{l}$, C. Wools ${ }^{7}$, J. Christodoulou ${ }^{1,2,3}$

${ }^{1}$ Murdoch Children's Res Inst, Melbourne, Australia, ${ }^{2}$ University of Melbourne, Melbourne, Australia, ${ }^{3}$ Victorian Clinical Genetics Services, Melbourne, Australia, ${ }^{4}$ Sydney Children's Hospitals Network, Sydney, Australia, ${ }^{5}$ Women's and Children's Hospital, Adelaide, Australia, ${ }^{6}$ Queensland Children's Hospital and Wesley Hospital, Brisbane, Australia, ${ }^{7}$ Royal Melbourne Hospital, Melbourne, Australia, ${ }^{8}$ Royal Adelaide Hospital, Adelaide, Australia, ${ }^{9}$ Hunter Genetics, John Hunter Hospital, Newcastle, Australia, ${ }^{10}$ Bio21 Molecular Science and Biotechnology Institute, Melbourne, Australia, ${ }^{11}$ Perth Children's Hospital, Perth, Australia, ${ }^{12}$ Yale School of Medicine, New Haven, CT, United States, ${ }^{13}$ Royal Perth Hospital, Perth, Australia, ${ }^{14}$ Royal Children's Hospital, Melbourne, Australia, ${ }^{15}$ Monash Biomedicine Discovery Institute, Monash University, Melbourne, Australia, ${ }^{16}$ Westmead Hospital, 
Sydney, Australia, ${ }^{17}$ Tasmanian Clinical Genetics Service, Hobart, Australia

Introduction: The Australian Genomics Health Alliance is a nationally funded health services research project focused on bringing genomic technologies to the clinic. The Mitochondrial Disease (MD) Flagship brings together expert clinicians from around Australia, specialist diagnostic laboratories and researchers expert in functional characterization of MD to examine the clinical utility of genomic sequencing for efficient diagnosis of MD.

Methods: We recruited 134 paediatric and adult individuals with a probable or definite MD diagnosis, based on modified Nijmegen criteria. Their blood DNA was randomized to either whole genome sequencing or whole exome plus mitochondrial DNA sequencing.

Results: Our definitive diagnostic yield is currently $\sim 36 \%$ for both whole genome and whole exome/ mitochondrial DNA approaches, with most diagnosed cases having a primary defect in the nuclear genome. Not surprisingly, $14 \%$ of individuals with confirmed molecular diagnoses had mutations in non-MD genes, reflecting phenotypic overlap. Unsolved patients have been triaged to one or more targeted functional tests, quantitative proteomics, RNAseq or long-read genome sequencing. This enabled re-categorising of numerous class 3 variants and identification of novel disease genes and mechanisms, including a de novo duplication within the ATAD3 locus resulting in a presumably toxic fusion protein (see Frazier et al. abstract).

Conclusions: Our collaborative effort has demonstrated that using a consistent national approach for clinically triaging cases for genomic sequencing is both effective and efficient, usually obviates the need for invasive biopsies and in some cases enables targeted therapies. Supported by grants from an NHMRC Targeted Call for Research (GNT1113531) and the Mito Foundation.

D. Thorburn: None. N. Baker: None. S. Balasubramaniam: None. D. Bratkovic: None. D. Coman: None. A. Compton: None. M. Delatycki: None. C. Ellaway: None. M. Fahey: None. J. Fletcher: None. A. Frazier: None. R. Ghaoui: None. H. Goel: None. D. Hock: None. M. Kava: None. N. Lake: None. P. Lamont: None. J. Lee: None. J. Panetta: None. L. Phillips: None. R. Rius: None. M. Ryan: None. N. Smith: None. D. Stroud: None. M. Tchan: None. M. Walsh: None. M. Wallis: None. A. Welch: None. C. Wools: None. J. Christodoulou: None.

\section{C09.2}

High resolution respirometric analysis of a Barth Syndrome disease model
G. Oemer $^{l *}$, K. Lackner ${ }^{l}$, J. Koch ${ }^{l}$, E. R. Werner ${ }^{l}$, C. Doerrier $^{2}$, G. Krumschnabel ${ }^{2}$, G. Leman ${ }^{l}$, S. Dubrac ${ }^{l}$, R. Houtkooper $^{3}$, J. Zschocke ${ }^{1}$, M. A. Keller ${ }^{1}$

${ }^{I}$ Medical University of Innsbruck, Innsbruck, Austria, ${ }^{2}$ Oroboros Instruments, Innsbruck, Austria, ${ }^{3}$ Amsterdam Medical Center, Amsterdam, Netherlands

Barth Syndrome is a rare $\mathrm{X}$-linked disorder that is characterized by mitochondrial dysfunction leading to severe cardiomyopathy, growth delay, muscle weakness and neutropenia. The affected $T A Z$ gene encodes the CoA-independent transacylase tafazzin that remodels the acyl chains of cardiolipins. This phospholipid, which is synthesized and localized in mitochondrial membranes, is functionally involved in cristae formation, protein anchoring, supercomplex assembly, ROS scavenging and apoptotic signaling. Cardiolipin has a unique structure with four fatty acyls rendering cardiolipins the most diverse phospholipid class. We generated a Barth Syndrome disease model by CRISPR/Cas9mediated HEK TAZ-knockout cell lines and have thoroughly characterized the induced alterations on cardiolipin homeostasis and mitochondrial functions. Furthermore we applied a recently developed LC-MS/MS lipidomics method to qualitatively, quantitatively and structurally characterize changes in the cardiolipin composition. In combination with systematic alteration of the lipid environment by BSA-mediated fatty acid supplementation, we obtained new insights in the complex mitochondrial - and partially rescuable - phenotypes of Barth Syndrome. As such, we observed fatty acid dependent mitochondrial fission and oxidative stress in TAZ-knockouts. Most strikingly, high-resolution respirometry revealed a shift in the OXPHOS flux control from NADH-depended to succinate-linked pathways without affecting the mitochondrial central carbon metabolic network or B-oxidation. This indicates a compensatory adaptation caused by the altered mitochondrial lipid homeostasis in TAZ-deficient cells.

G. Oemer: None. K. Lackner: None. J. Koch: None. E. R. Werner: None. C. Doerrier: None. G. Krumschnabel: None. G. Leman: None. S. Dubrac: None. R. Houtkooper: None. J. Zschocke: None. M. A. Keller: None.

\section{C09.3}

Recurrent de novo ATAD3 duplications cause fatal perinatal mitochondrial cardiomyopathy, persistent hyperlactacidemia, encephalopathy and heart-specific mitochondrial oxidative phosphorylation complex I deficiency.

\author{
A. E. Frazier ${ }^{1,2}$, A. G. Compton ${ }^{1,2 *}$, Y. Kishita ${ }^{3}$, D. H. \\ Hock $^{4}$, A. E. Welch ${ }^{1}$, S. S. C. Amarasekera ${ }^{1,2}$, R. Rius ${ }^{1,2}$, L. \\ E. Formosa ${ }^{5}$, A. Imai-Okazaki ${ }^{3,6}$, D. Francis ${ }^{7}$, M. Wang ${ }^{1}, N$. \\ J. Lake ${ }^{1,2,8}$, S. Tregoning ${ }^{1,7}$, J. S. Jabbari ${ }^{9}$, A. Lucattini ${ }^{9}, K$.
}




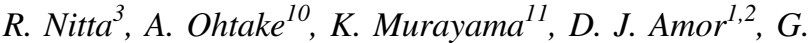
McGillivray ${ }^{7}$, F. Y. Wong ${ }^{12}$, M. S. van der Knaap ${ }^{13,14}$, R. J. Vermeulen $^{15}$, E. J. Wiltshire ${ }^{16}$, J. M. Fletcher ${ }^{17}$, B. Lewis ${ }^{18}$, G. Baynam ${ }^{19,20}$, C. Ellaway ${ }^{21,22}$, S. Balasubramaniam ${ }^{21}, K$. Bhattacharya $^{21,22}$, M. L. Freckmann ${ }^{23}$, R. J. Taft ${ }^{24}, S$. Sadedin $^{1,7}$, M. J. Cowley ${ }^{25,26}$, A. E. Minoche ${ }^{26}$, S. E. Calvo $^{27}$, V. K. Mootha ${ }^{27}$, M. T. Ryan ${ }^{5}$, Y. Okazaki , D. A. Stroud $^{4}$, C. Simons ${ }^{1,28}$, J. Christodoulou ${ }^{1,2,7}$, D. R. Thorburn ${ }^{1,2,7}$

${ }^{1}$ Murdoch Children's Research Institute, Melbourne, Australia, ${ }^{2}$ Department of Paediatrics, University of Melbourne, Melbourne, Australia, ${ }^{3}$ Diagnostics and Therapeutics of Intractable Diseases, Intractable Disease Research Center, Juntendo University, Graduate School of Medicine, Tokyo, Japan, ${ }^{4}$ Department of Biochemistry and Molecular Biology and Bio21 Molecular Science and Biotechnology Institute, University of Melbourne, Melbourne, Australia, ${ }^{5}$ Department of Biochemistry and Molecular Biology, Monash Biomedicine Discovery Institute, Monash University, Melbourne, Australia, ${ }^{6}$ Division of Genomic Medicine Research, Medical Genomics Center, National Center for Global Health and Medicine, Tokyo, Japan, ${ }^{7}$ Victorian Clinical Genetics Services, Murdoch Children's Research Institute, Royal Children's Hospital, Melbourne, Australia, ${ }^{8}$ Department of Genetics, Yale School of Medicine, New Haven, CT, United States, ${ }^{9}$ Australian Genome Research Facility Ltd, Victorian Comprehensive Cancer Centre, Melbourne, Australia, ${ }^{10}$ Department of Pediatrics \& Clinical Genomics, Saitama Medical University Hospital, Saitama, Japan, ${ }^{11}$ Department of Metabolism, Chiba Children's Hospital, Chiba, Japan, ${ }^{12}$ Ritchie Centre, Hudson Institute of Medical Research; Department of Paediatrics, Monash University; and Monash Newborn, Monash Children's Hospital, Melbourne, Australia, ${ }^{13}$ Child Neurology, Emma Children's Hospital, Amsterdam University Medical Centers, Vrije Universiteit and Amsterdam Neuroscience, Amsterdam, Netherlands, ${ }^{14}$ Functional Genomics, Center for Neurogenomics and Cognitive Research, Vrije Universiteit and Amsterdam Neuroscience, Amsterdam, Netherlands, ${ }^{15}$ Department of Neurology, Maastricht University Medical Center, Maastricht, Netherlands, ${ }^{16}$ Department of Paediatrics and Child Health, University of Otago Wellington and Capital and Coast District Health Board, Wellington, New Zealand, ${ }^{17}$ Department of Genetics and Molecular Pathology, SA Pathology, Adelaide, Australia, ${ }^{18}$ Department of Clinical Biochemistry, PathWest Laboratory Medicine Western Australia, Perth, Australia, ${ }^{19}$ Western Australian Register of Developmental Anomalies and Genetic Services of Western Australia and King Edward Memorial Hospital for Women Perth, Perth, Australia, ${ }^{20}$ Telethon Kids Institute and School of Paediatrics and Child Health, The University of Western Australia, Perth, Australia, ${ }^{21}$ Genetic Metabolic Disorders Service, Sydney Children's Hospital Network, The Children's Hospital at Westmead, Sydney, Australia, ${ }^{22}$ Disciplines of Genomic Medicine and Child and Adolescent Health, Sydney Medical School, University of Sydney, Sydney, Australia, ${ }^{23}$ Clinical Genetics, The Canberra Hospital, Canberra, Australia, ${ }^{24}$ Illumina, Inc., San Diego, CA, United States, ${ }^{25}$ Children's Cancer Institute, and St Vincent's Clinical School, UNSW Sydney, Sydney, Australia, ${ }^{26}$ Kinghorn Centre for Clinical Genomics, Garvan Institute of Medical Research, Sydney, Australia, ${ }^{27}$ Broad Institute; Howard Hughes Medical Institute and Department of Molecular Biology, Massachusetts General Hospital; and Harvard Medical School, Boston, MA, United States, ${ }^{28}$ Institute for Molecular Bioscience, The University of Queensland, Brisbane, Australia

Mitochondrial disorders are clinically heterogeneous and comprise over 350 different genetic conditions. However, the molecular diagnosis is unknown in $\sim 50 \%$ of cases, partly due to some genomic regions being refractory to standard genomic analysis. One such region is the ATAD3 locus consisting of 3 highly homologous tandemly arrayed genes ( $A T A D 3 C, A T A D 3 B$ and $A T A D 3 A$ ) encoding mitochondrial proteins implicated in processes including cholesterol metabolism, and mitochondrial replication, dynamics and morphology. Recessive deletions and dominant duplications in this locus have recently been reported to cause rare, lethal perinatal mitochondrial disorders characterised by pontocerebellar hypoplasia or cardiomyopathy, respectively. We report 17 subjects from 16 unrelated families with cardiomyopathy, persistent hyperlactacidemia, encephalopathy and frequently corneal clouding or cataracts due to de novo ATAD3 duplications. The six different $68 \mathrm{~Kb}$ duplications were consistently identifiable from whole genome and exome sequencing, but usually missed on microarray. The duplications all resulted in the formation of an identical chimeric ATAD3A/ATAD3C fusion protein, which appears to act in a dominant manner causing altered ATAD3 complexes and a striking reduction in mitochondrial oxidative phosphorylation complex I and its activity in heart tissue. In our experience, the ATAD3 locus is one of the five most common causes of nuclear-encoded paediatric mitochondrial disease but the repetitive nature of the locus means ATAD3 diagnoses may be frequently missed by current genomic strategies. Supported by grants from the Australian NHMRC 1164479 and 1113531, US DoD CDMRP PR170396; the Japanese AMED 19ek0109273, 18ek0109177, 19kk0205014 and 18kk0205002; Japanese JSPS 19H03624 and the Australian Mito Foundation. 
A. E. Frazier: None. A. G. Compton: None. Y. Kishita: None. D. H. Hock: None. A. E. Welch: None. S. S. C. Amarasekera: None. R. Rius: None. L. E. Formosa: None. A. Imai-Okazaki: None. D. Francis: None. M. Wang: None. N. J. Lake: None. S. Tregoning: None. J. S. Jabbari: None. A. Lucattini: None. K. R. Nitta: None. A. Ohtake: None. K. Murayama: None. D. J. Amor: None. G. McGillivray: None. F. Y. Wong: None. M. S. van der Knaap: None. R. J. Vermeulen: None. E. J. Wiltshire: None. J. M. Fletcher: None. B. Lewis: None. G. Baynam: None. C. Ellaway: None. S. Balasubramaniam: None. K. Bhattacharya: None. M. L. Freckmann: None. R. J. Taft: A. Employment (full or part-time); Significant; Illumina, Inc. S. Sadedin: None. M. J. Cowley: None. A. E. Minoche: None. S. E. Calvo: None. V. K. Mootha: None. M. T. Ryan: None. Y. Okazaki: None. D. A. Stroud: None. C. Simons: None. J. Christodoulou: None. D. R. Thorburn: None.

\section{C09.4}

Hyperuricemia and gout caused by missense mutation in D-lactate dehydrogenase, revealing novel enzymatic activity

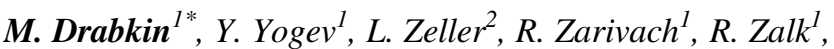
D. Halperin ${ }^{1}$, O. Wormser ${ }^{1}$, E. Gurevich ${ }^{3}$, D. Landau ${ }^{4}, R$.

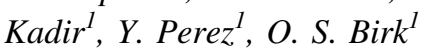

${ }^{1}$ Ben-Gurion University, Beer-Sheva, Israel, ${ }^{2}$ Soroka Medical Center, Beer-Sheva, Israel, "Clalit" Health Services, Beer-Sheva, Israel, ${ }^{4}$ Tel-Aviv University, Tel-Aviv, Israel

Introduction: Gout is caused by deposition of monosodium urate crystals in joints when plasma uric acid levels are elevated beyond saturation threshold, mostly due to renal under-excretion of uric acid. While molecular pathways of this under-excretion have been elucidated, its etiology remains mostly unknown. We studied a consanguineous Bedouin kindred affected with gout and hyperuricemia.

Materials and Methods: Whole-exome sequencing, linkage analysis, protein structure modeling, mass-spectrometry, metabolomics, confocal microscopy, in-vivo metabolic studies, co-immunoprecipitation, proteomics, knockout mouse model.

Results: We demonstrate that gout can be caused by a mutation in $L D H D$ within the putative catalytic site of the encoded D-lactate dehydrogenase, resulting in augmented blood and urine levels of D-lactate, a stereoisomer of Llactate, normally present in human blood in miniscule amounts. Consequent excessive renal secretion of D-lactate in exchange for uric acid reabsorption culminated in hyperuricemia and gout. Metabolome analysis of patient's blood and urine using mass-spectrometry revealed elevated levels of intermediate metabolites of purine degradation, which ultimately lead to uric acid production. Further metabolome analyses performed on $L D H D$ knockout mice generated using CRISPR-Cas9 recapitulated the human phenotype. We showed that $L D H D$ expression is enriched in tissues with high metabolic rate and abundant mitochondria, and that D-lactate dehydrogenase resides in the mitochondria of cells overexpressing the human $L D H D$ gene. In line with the human phenotype, injection of Dlactate to naïve mice resulted in hyperuricemia.

Conclusion: Hyperuricemia and gout can result from accumulation of D-lactate and possibly additional metabolites whose renal excretion is coupled to uric acid reabsorption.

M. Drabkin: None. Y. Yogev: None. L. Zeller: None. R. Zarivach: None. R. Zalk: None. D. Halperin: None. O. Wormser: None. E. Gurevich: None. D. Landau: None. R. Kadir: None. Y. Perez: None. O. S. Birk: None.

\section{C09.5}

Metabolite set enrichment improves biomarker identification and detection in untargeted metabolic profiling (UMP) data for patients with inborn errors of metabolism

\section{B. Hoegen ${ }^{*}$, U. F. H. Engelke ${ }^{2}$, K. L. M. Coene ${ }^{2}$, J. E. Hampstead $^{1}$, P. Kulkarni ${ }^{2}$, R. A. Wevers ${ }^{2}$, H. G. Brunner ${ }^{3}$, C. Gilissen ${ }^{1}$}

${ }^{I}$ Department of Human Genetics, Radboud Institute for Molecular Life Sciences, Radboud University Medical Center, Nijmegen, Netherlands, ${ }^{2}$ Department of Laboratory Medicine, Translational Metabolic Laboratory (TML), Radboud University Medical Center, Nijmegen, Netherlands, ${ }^{3}$ Department of Human Genetics, Donders Center for Neuroscience, Radboud University Medical Center, Nijmegen, Netherlands

Inborn errors of metabolism (IEMs) are a large, predominantly recessive class of genetic disorders associated with the congenital disruption of one or more metabolic pathways. Untargeted metabolomic profiling (UMP) allows the simultaneous measurement of hundreds of metabolites, including diagnostic biomarker metabolites for IEM disorders, in a single test and can aid in the interpretation of variants of uncertain significance (VUS) in genes with a known or suspected metabolic phenotype. However, the large amount of data generated in a single UMP run limits its diagnostic utility. Here, we present a metabolite set enrichment method (MSEA) that leverages biological pathway information to prioritise clinically relevant metabolites and their associated pathways in UMP data. We further cluster enriched pathways into pathway sets to eliminate redundancy. We ran MSEA across 43 diagnosed 
patient plasma samples with pathway information available in databases $(\mathrm{KEGG}=317, \mathrm{SMPDB}=901)$ spanning 21 distinct IEMs, and show that MSEA prioritises known diagnostic biomarkers better than a naive filtering approach in 18 samples across 10 IEMs without a genetic prior $(\mathrm{p}=0.00015)$. This has particular relevance for patients with unresolved IEMs, or novel IEMs not included on standard gene panels. Additionally, our method identifies putative novel biomarkers for at least one IEM. MSEA improves the analysis of UMP data for patient diagnostics, and provides a first approach to opening the metabolome. These approaches will be crucial for the interpretation of variants of unknown significance from WES data, and for the integration of WES and WGS results with untargeted metabolomics data.

B. Hoegen: None. U. F. H. Engelke: None. K. L. M. Coene: None. J. E. Hampstead: None. P. Kulkarni: None. R. A. Wevers: None. H. G. Brunner: None. C. Gilissen: None.

\section{C09.6}

A genome-wide association study for a proxy nonalcoholic fatty liver phenotype identifies novel loci and trait-relevant candidate genes

M. Vujkovic ${ }^{1,2}$, S. Ramdas ${ }^{3}$, K. Gawronski ${ }^{4}$, K. Lorenz $^{1,3,5}$, M. Serper ${ }^{1,2,6}$, D. E. Kaplan ${ }^{1,2}$, R. Carr ${ }^{1,2}$, K. M. Lee ${ }^{7}$, S.

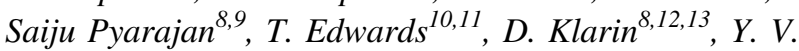
Sun $^{14,15}$,D. R. Miller ${ }^{16,17}$, P. D. Reaven ${ }^{18,19}$, L. S. Phillips $^{14,20}$, C. J. O'Donnell ${ }^{8,9}$, J. B. Meigs ${ }^{21,22,23}$, P. W. F. Wilson $^{24,25}$, R. Vickers-Smith ${ }^{1,26}$, H. R. Kranzler ${ }^{1,27}$, A. C.

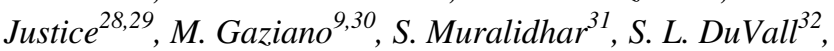
T. L. Assimes ${ }^{33,34}$, J. S. Lee L3,34, P. S. Tsao $^{33,34}$, D. J. Rader $^{2,3,35}$, C. D. Brown ${ }^{3}$ S. M. Damrauer ${ }^{1,36}$, J. A. Lynch $^{32,37}$, D. Saleheen ${ }^{38,39}$, B. F. Voight ${ }^{1,3,5^{*}}, K$. M. Chang $^{1,2}$, on behalf of the VA Million Veteran Program

${ }^{1}$ Corporal Michael J. Crescenz VA Medical Center, Philadelphia, PA, United States, ${ }^{2}$ Department of Medicine, University of Pennsylvania, Philadelphia, PA, United States, ${ }^{3}$ Department of Genetics, University of Pennsylvania, Philadelphia, PA, United States, ${ }^{4}$ Cellular and Molecular Biology Graduate Group, University of Pennsylvania, Philadelphia, PA, United States, ${ }^{5}$ Department of Systems Pharmacology and Translational Therapeutics, University of Pennsylvania, Philadelphia, PA, United States, ${ }^{6}$ Leonard Davis Institute of Health Economics, Philadelphia, PA, United States, ${ }^{7}$ VA Salt Lake City Health Care System, Salt Lake City, UT, United States, ${ }^{8}$ VA Boston Healthcare System, Boston, MA, United States, ${ }^{9}$ Department of Medicine, Brigham Women's Hospital, Boston, MA, United States, ${ }^{10}$ Nashville VA Medical Center, Philadelphia, PA, United States, ${ }^{11}$ Vanderbilt Genetics Institute, Vanderbilt University Medical Center, Nashville, TN,
United States, ${ }^{12}$ Center for Genomic Medicine, Massachusetts General Hospital, Boston, MA, United States, ${ }^{13}$ Division of Vascular Surgery and Endovascular Therapy, University of Florida School of Medicine, Gainesville, FL, United States, ${ }^{14}$ Atlanta VA Health Care System, Atlanta, GA, United States, ${ }^{15}$ Department of Epidemiology, Emory University Rollins School of Public Health, Atlanta, GA, United States, ${ }^{16}$ Edith Nourse Rogers Memorial VA Hospital, Bedford, MA, United States, ${ }^{17}$ Department of Health Law, Policy and Management, Boston University School of Public Health, Boston, MA, United States, ${ }^{18}$ Phoenix VA Health Care System, Pheonix, AZ, United States, ${ }^{19}$ College of Medicine, University of Arizona, Tuscon, AZ, United States, ${ }^{20}$ Division of Endocrinology, Emory University School of Medicine, Atlanta, GA, United States, ${ }^{21}$ Division of General Internal Medicine, Massachusetts General Hospital, Boston, MA, United States, ${ }^{22}$ Department of Medicine, Harvard Medical School, Boston, MA, United States, ${ }^{23}$ Broad Institute of MIT and Harvard, Boston, MA, United States, ${ }^{24}$ Atlanta VA Medical Center, Atlanta, GA, United States, ${ }^{25}$ Division of Cardiology, Emory University School of Medicine, Atlanta, GA, United States, ${ }^{26}$ University of Louisville, Louisville, $K Y$, United States, ${ }^{27}$ Department of Psychiatry, University of Pennsylvania, Philadelphia, PA, United States, ${ }^{28}$ Veterans Affairs Connecticut Healthcare System, West Haven, CT, United States, ${ }^{29}$ Yale School of Medicine and Yale School of Public Health, New Haven, CT, United States, ${ }^{30}$ Massachusetts Veterans Epidemiology Research and Information Center, Veterans Affairs Boston Healthcare System, Boston, MA, United States, ${ }^{31}$ Office of Research and Development, Veterans Health Administration, Washington, DC, United States, ${ }^{32}$ VA Informatics and Computing Infrastructure, VA Salt Lake City Health Care System, Salt Lake City, UT, United States, ${ }^{33}$ VA Palo Alto Health Care System, Palo Alto, CA, United States, ${ }^{34}$ Department of Medicine, Stanford University, Stanford, CA, United States, ${ }^{35}$ Department of Pediatrics, University of Pennsylvania, Philadelphia, PA, United States, ${ }^{36}$ Department of Surgery, University of Pennsylvania, Philadelphia, PA, United States, ${ }^{37}$ University of Massachusetts College of Nursing and Health Sciences, Boston, MA, United States, ${ }^{38}$ Department of Medicine and Cardiology, Columbia University Irving Medical Center, New York, NY, United States, ${ }^{39}$ Center for Non-Communicable Diseases, Karachi, Pakistan

Introduction: Non-alcoholic fatty liver disease (NAFLD) is a prevalent, heritable trait that can progress to cirrhosis, liver failure, and cancer. Elucidation of its etiology has been stymied by the lack of human genomics data at scale. We previously reported a proxy phenotype for NAFLD: elevated serum alanine aminotransferase levels across multiple time 
points without alternate causes of liver disease, recovering all previously associated variants. Using this definition, we performed genome-wide association in a cohort of United States Veteran participants in the Million Veteran Program.

Methods: Trans-ethnic meta-analysis included 95,940 cases and 145,858 controls of European, African, Asian, and Latinx ancestries. Gene prioritization was based on linkage with coding variants or co-localization (COLOC $\mathrm{P} 4>0.8$ ) with quantitative trait loci (QTL) from GTEx. Enrichment analyses were performed via GREGOR and DEPICT; heritability analyses via LD-score regression.

Results: 75 loci exceeded genome-wide significance, with 3 additional loci identified in an African-American specific scan. Candidate genes at 34 loci were prioritized based on coding or QTL variation tightly linked with lead variants, with 9 variants predicted damaging or had reduced expression associated with lower incidence of our proxy trait. Total liability scale $\mathrm{h}^{2}$ was estimated at $16 \%$ (95\% CI: 12-19\%). Functional genomic annotations and gene-sets demonstrated enrichment of liver biology at associated sites. Causal inference analyses indicated that body mass index (BMI) and waist-hip ratio adjusted for BMI were associated with our proxy trait $\left(\mathrm{P}<10^{-10}\right)$.

Conclusion: We identified novel loci with 34 candidate genes plausibly associated with non-alcoholic fatty liver disease.

M. Vujkovic: None. S. Ramdas: None. K. Gawronski: None. K. Lorenz: None. M. Serper: None. D. E. Kaplan: None. R. Carr: None. K. M. Lee: None. S. Saiju Pyarajan: None. T. Edwards: None. D. Klarin: None. Y. V. Sun: None. D. R. Miller: None. P. D. Reaven: None. L. S. Phillips: None. C. J. O'Donnell: None. J. B. Meigs: None. P. W. F. Wilson: None. R. Vickers-Smith: None. H. R. Kranzler: None. A. C. Justice: None. M. Gaziano: None. S. Muralidhar: None. S. L. DuVall: None. T. L. Assimes: None. J. S. Lee: None. P. S. Tsao: None. D. J. Rader: None. C. D. Brown: None. S. M. Damrauer: None. J. A. Lynch: None. D. Saleheen: None. B. F. Voight: None. K. M. Chang: None.

\section{C10 Rare and Common Variants in Personalized Medicine}

\section{C10.1}

Recessive vs dominant GWAS of 82,516 coding variants on electronic health records in a population-wide analysis of 176,899 Finns

H. O. Heyne ${ }^{1,2,3^{*}}$, J. Karjalainen ${ }^{1,2,3}$, S. M. Lemmelä $\ddot{A}^{1,4}$, FinnGen, A. S. Havulinna ${ }^{1,4}$, M. Kurki ${ }^{1,2,3}$, A. Palotie ${ }^{1,2,3}$, M. J. Daly ${ }^{1,2,3}$

${ }^{1}$ Institute for Molecular Medicine Finnland: FIMM, Helsinki, Finland, ${ }^{2}$ Analytic and Translational Genetics Unit,
Massachusetts General Hospital, Boston, MA, United States, ${ }^{3}$ Broad Institute of MIT and Harvard, Cambridge, MA, United States, ${ }^{4}$ Finnish Institute for Health and Welfare, Helsinki, Finland

Rare genetic variants influencing diseases often have large effect sizes with potential direct treatment implications; studying their effects comprehensively requires however large cohort sizes. The population-wide cohort of FinnGen offers an unprecedented opportunity to investigate phenotype associations of rare variants because the founding bottleneck of Finland concentrates them to higher population frequencies. This 'Finnish disease heritage' applies particularly to recessive variants. Here, we studied recessive, dominant and additive effects of 82,516 coding variants on 2,264 disease endpoints using nation wide electronic health record data of 176,899 Finns. Globally, variant effects in different GWAS models were in agreement with inheritance modes of the respective genes in OMIM (recessive, dominant or "both"). However, there were many exceptions e.g. 33 genes listed only as recessive in OMIM had coding variants with dominant associations $\left(\mathrm{p}<10^{-7}\right)$. One example was XPA (recessive disease: Xeroderma pigmentosa), where a heterozygous truncating variant was associated with skin cancer $\left(\mathrm{p}=8 \times 10^{-11}\right.$, beta $=$ 1.24). Another example was a variant in $S C N 5 A$ associated with sick sinus syndrome in recessive state. Interestingly, in heterozygous form it had a protective effect for cardiac arrhythmia phenotypes $\left(\mathrm{p}=2 \times 10^{-8}\right.$, beta $=-0.48$, replicated in UK Biobank with $\mathrm{p}=0.04$, beta $=-0.39$ ). Our data also included 6794 coding variants listed in ClinVar. We found significantly more phenotype associations than for random intergenic variants in 498 (likely) pathogenic/conflicting, but also 4019 "benign" variants in dominant and recessive genes. We report new findings from associations of coding variants with population wide electronic health record data in FinnGen.

H. O. Heyne: None. J. Karjalainen: None. S. M. Lemmelä: None. A. S. Havulinna: None. M. Kurki: None. A. Palotie: None. M. J. Daly: E. Ownership Interest (stock, stock options, patent or other intellectual property); Modest; Maze Therapeutics.

\section{C10.2}

Germline genetic testing following tumor sequencing in cancer patients has a remarkably high yield of clinically important findings that inform patient care

\author{
S. Lincoln ${ }^{*}$, K. Das, N. Ngo, S. Michalski, S. Yang, D. \\ Pineda, E. Esplin, S. Aradaya, R. Nussbaum
}

Invitae, San Francisco, CA, United States 
Introduction: Recent ESMO guidelines recommend germline genetic testing following tumor sequencing, but only for certain genes in specific patients (PMID 31050713). We examined the utility of germline findings in patients receiving both test types.

Methods: 1849 consecutive patients were analyzed, who: (a) received germline testing, and (b) previously had received tumor sequencing. Indications for germline testing were diverse and included: tumor findings of potential germline origin, treatment/surgical planning, personal/ family history, and patient concern. 238 patients had colorectal cancer, 227 breast, 208 prostate, 189 lung, 173 pancreas, and the remainder had other cancers. $12 \%$ had multiple primary diagnoses.

Results: $31 \%$ of patients harbored a high or moderate penetrance pathogenic germline variant (PGV) in a known cancer predisposition gene. Mutations in certain genes were more likely to be germline compared to others (Table) and PGVs were prevalent among most cancer types studied. Most PGVs were potentially actionable, but $25 \%$ of PGVs failed to meet ESMO criteria for germline testing. $8.6 \%$ of $\mathrm{PGVs}$ were not reported by tumor sequencing as either somatic or germline findings, often because of test limitations. $9.3 \%$ of patients had PGVs identified only after presenting with a second, possibly preventable, cancer.

Conclusions: Germline testing following tumor sequencing often produces clinically important findings. Our results suggest that such testing may however be underutilized and that guidelines for reflex testing appear effective although conservative.

Germline and Somatic Findings in 1849 Patients (Genes with no germline findings are not listed)

\begin{tabular}{lllll}
\hline Genes & $\begin{array}{l}\text { Total } \\
\text { Findings }\end{array}$ & $\begin{array}{l}\text { \#Germline } \\
\text { (\%Findings) }\end{array}$ & $\begin{array}{l}\text { Met ESMO } \\
\text { criteria? }\end{array}$ & $\begin{array}{l}\text { Potential } \\
\text { Utility }\end{array}$ \\
\hline BRCA1/2 & 765 & $295(39 \%)$ & Yes & MG, PT, CT \\
ATM & 136 & $58(43 \%)$ & No & MG, CT \\
MLH1, MSH2, & 182 & $55(30 \%)$ & Yes & MG, PT, CT \\
MSH6, PMS2 & & & & \\
CHEK2 & 68 & $49(72 \%)$ & No & MG, CT \\
PALB2 & 60 & $44(73 \%)$ & Yes & MG, CT \\
BRIP1 & 24 & $10(42 \%)$ & Yes & MG, CT \\
TP53 & 357 & $14(4 \%)$ & $10 / 14$ No* & MG, CT \\
RET & 23 & $8(35 \%)$ & Yes & MG, CT \\
BAP1 & 33 & $7(21 \%)$ & $3 / 7$ No* & MG, CT \\
CDKN2A & 105 & $6(6 \%)$ & No & MG \\
NF1 & 41 & $6(15 \%)$ & Yes & MG \\
SDHA & 13 & $5(39 \%)$ & Yes & MG \\
FH & 6 & $5(83 \%)$ & $3 / 5$ No* & MG
\end{tabular}

(continued)

Germline and Somatic Findings in 1849 Patients (Genes with no germline findings are not listed)

\begin{tabular}{lllll}
\hline Genes & $\begin{array}{l}\text { Total } \\
\text { Findings }\end{array}$ & $\begin{array}{l}\text { \#Germline } \\
(\% \text { Findings })\end{array}$ & $\begin{array}{l}\text { Met ESMO } \\
\text { criteria? }\end{array}$ & $\begin{array}{l}\text { Potential } \\
\text { Utility }\end{array}$ \\
\hline$N B N$ & 11 & $5(46 \%)$ & No & MG, CT \\
$C D H 1$ & 67 & $4(6 \%)$ & No & MG \\
RAD51C & 4 & $4(100 \%)$ & Yes & MG, CT \\
Others & 312 & $28(9 \%)$ & varies & varies \\
\hline
\end{tabular}

*ESMO criteria for this gene specify patient age and/or tumor types **MG: Management Guidelines, PT: Precision Therapy (FDA), CT: Clinical Trials

S. Lincoln: A. Employment (full or part-time); Significant; Invitae. E. Ownership Interest (stock, stock options, patent or other intellectual property); Significant; Illumina, Thermo Fisher. K. Das: A. Employment (full or part-time); Significant; Invitae. E. Ownership Interest (stock, stock options, patent or other intellectual property); Modest; Amarin Dx. F. Consultant/Advisory Board; Modest; Amarin Dx. N. Ngo: A. Employment (full or part-time); Significant; Invitae. S. Michalski: A. Employment (full or part-time); Significant; Invitae. S. Yang: A. Employment (full or part-time); Significant; Invitae. D. Pineda: A. Employment (full or part-time); Significant; Invitae. E. Esplin: A. Employment (full or part-time); Significant; Invitae. S. Aradaya: A. Employment (full or part-time); Significant; Invitae. R. Nussbaum: A. Employment (full or part-time); Significant; Invitae.

\section{C10.3}

The first genotype-phenotype study on European carriers of $\mathrm{CDH} 1$ germline mutations

J. García-Peláez ${ }^{l *}$, R. Monteiro ${ }^{l}$, L. Sousa ${ }^{l}$, H. Pinheiro ${ }^{l}$, S. Castedo ${ }^{1,2,3}$, L. Garrido ${ }^{2}$, M. Teixeira ${ }^{4,5}$, G. Michils $^{6}$, V. Bours $^{7,8,5}$, R. de Putter ${ }^{9,5}$, L. Golmard \& M. Blanluet ${ }^{10}, C$. Colas $^{10,5}$, P. Benusiglio ${ }^{11}$, S. Aretz \& I. Spier ${ }^{12,13,5}, R$. Hüneburg ${ }^{13,14}$, L. Gieldon ${ }^{15,16}$, E. Schröck ${ }^{15,16,5}$, E. Holinski-Feder \& V. Steinke ${ }^{17,18,5}$, D. Calistri \& G. Tedaldi $^{19}$, G. Ranzani ${ }^{20}$, M. Genuardi ${ }^{21,22}$, C. Silveira \& I. Silva ${ }^{23}$, M. Krajc \& A. Blatnik ${ }^{24,5}$, S. Novakovic ${ }^{24}$, A. Patiño-García ${ }^{25}$, J. Soto ${ }^{26}$, C. Lázaro ${ }^{27,5}$, G. Capelláa ${ }^{27,5}, J$. Brunet-Vidal $^{28,5}$, J. Balmaña ${ }^{29,5}$, E. Domínguez-Garrido ${ }^{30}$, M. Ligtenberg ${ }^{31,5}$, E. Fewings $^{32}$, R. Fitzgerald Fi $^{33}, E$. Woodward $^{34,35,5}$, G. Evans ${ }^{36,5}$, H. Hanson ${ }^{37}$, K. LagerstedtRobinson $^{38}$, S. Bajalica-Lagercrantz ${ }^{38,5}$, C. Egas ${ }^{39}, C$. Martínez-Bouzas et al. ${ }^{40,41}, K$. Dahan \& D. Feret ${ }^{42}, N$. Hoogerbrugge $^{31,5}$, M. Tischkowitz ${ }^{32,5}$, C. Oliveira ${ }^{43}$ 
${ }^{1}$ IPATIMUP/i3S, Porto, Portugal, ${ }^{2}$ Centro Hospitalar Universitário São João (CHSJ), Porto, Portugal, ${ }^{3}$ Faculty of Medicine of the University of Porto (FMUP), Porto, Portugal, ${ }^{4}$ IPO, Instituto Português de Oncologia do Porto, Porto, Portugal, ${ }^{5}$ European Reference Network on Genetic Tumour Risk Syndromes (ERN GENTURIS), Nijmegen, Netherlands, ${ }^{6}$ Centre for Human Genetics, KU Leuven, Leuven, Belgium, ${ }^{7}$ Laboratory of Human Genetics, GIGAInstitute, University of Liège, Liège, Belgium, ${ }^{8}$ Center of Genetics, University Hospital (CHU), Liège, Belgium, ${ }^{9}$ Clinical Genetics department, University Hospital of Ghent, Ghent, Belgium, ${ }^{10}$ Genetic Department, Institut Curie, Paris, France, ${ }^{11}$ Département de Génétique, GH Pitié-Salpêtrière, Paris, France, ${ }^{12}$ Institute of Human Genetics, University of Bonn, Bonn, Germany, ${ }^{13}$ National Center for Hereditary Tumor Syndromes, University of Bonn, Bonn, Germany, ${ }^{14}$ Department of Internal Medicine I, University Hospital Bonn, Bonn, Germany, ${ }^{15}$ Institut für Klinische Genetik, Medizinische Fakultät Carl Gustav Carus, Dresden, Technische Universität Dresden, Dresden, Germany, ${ }^{16}$ Core Unit for Molecular Tumordiagnostics (CMTD), National Center for Tumor Diseases (NCT) site Dresden, German Cancer Research Center (DKFZ)Heidelberg, German Cancer Consortium (DKTK), Dresden, Germany, ${ }^{17}$ Medizinische Klinik und Poliklinik IV, Campus Innenstadt, Klinikum der Universität München, Ziemssenstr, Münich, Germany, ${ }^{18} M G Z$ - Medizinisch Genetisches Zentrum, Münich, Germany, ${ }^{19}$ Biosciences Laboratory, Istituto Scientifico Romagnolo per lo Studio e la Cura dei Tumori (IRST) IRCCS, Meldola, Italy, ${ }^{20}$ Department of Biology and Biotechnology "Lazzaro Spallanzani", University of Pavia, Pavia, Italy, ${ }^{21}$ Fondazione Policlinico Universitario "A. Gemelli" IRCCS, UOC di Genetica Medica, Rome, Italy, ${ }^{22}$ Istituto di Medicina Genomica, Università Cattolica del Sacro Cuore, Rome, Italy, ${ }^{23}$ Genomed Diagnósticos de Medicina Molecular, S. A., Lisbon, Portugal, ${ }^{24}$ Institute of Oncology Ljubljana, Department of Molecular Diagnostics, Ljubljana, Slovenia, ${ }^{25}$ Clinical Genetics Unit and CIMA LAB Diagnostics. University Clinic of Navarra, Pamplona, Spain, ${ }^{26}$ Molecular Genetics Laboratory, Elche University Hospital, Elche, Spain, ${ }^{27}$ Catalan Institute of Oncology, IDIBELL, CIBERONC, Barcelona, Spain, ${ }^{28}$ Catalan Institute of Oncology, IDIBELL, IDIBGI, Barcelona-Girona, Portugal, ${ }^{29}$ Hospital Vall d'Hebron, Barcelona, Spain, ${ }^{30}$ Molecular Diagnostics Laboratory, Fundación Rioja Salud, Logroño, Spain, ${ }^{31}$ Department of Human Genetics, Radboud University Medical Center and Radboud Institute for Molecular Life Sciences, Nijmegen, Netherlands, ${ }^{32}$ Academic Laboratory of Medical Genetics. National Institute for Health Research Cambridge Biomedical Research Centre, Cambridge, UK University of Cambridge, Cambridge, United Kingdom, ${ }^{33}$ MRC Cancer Unit, Hutchison-MRC Research Centre,
University of Cambridge, Cambridge, United Kingdom, ${ }^{34} \mathrm{NW}$ Genomic Laboratory hub, Manchester Centre for Genomic Medicine, Manchester University Hospitals NHS Foundation Trust, Manchester, United Kingdom, ${ }^{35}$ Division of Evolution and Genomic Sciences, School of Biological Sciences, Faculty of Biology, Medicine and Health, University of Manchester, Manchester Academic Health Science Centre, Manchester, United Kingdom, ${ }^{36}$ Manchester Centre for Genomic Medicine, Manchester Centre for Genomic Medicine, Manchester, United Kingdom, ${ }^{37} \mathrm{St}$ Georges NHS Foundation Trust, London, United Kingdom, ${ }^{38}$ Department of Molecular Medicine and Surgery, Karolinska Institutet and Department of Clinical Genetics, Karolinska Univ Hospital, Stockholm, Sweden, ${ }^{39}$ Advanced Services Unit, Biocant - Biotechnology Innovation Center, Cantanhede, Portugal, ${ }^{40}$ Biocruces Health Research Institute, Barakaldo, Spain, ${ }^{41}$ Molecular Genetics Laboratory, Genetics Service, Cruces University Hospital, Barakaldo, Spain, ${ }^{42}$ Institut de Génétique et de Pathologie, IPG, Gosselies, Belgium, ${ }^{43} i 3 S$ \& Ipatimup \& FMUP \& ERNGENTURIS \& CDH1-ClinGen working Group, Porto, Portugal

Hereditary Diffuse Gastric Cancer-HDGC syndrome, predisposing for diffuse gastric cancer-DGC and lobular breast cancer-LBC, is caused by germline $\mathrm{CDH} 1$ Pathogenic-P and Likely Pathogenic-LP variants. Surveillance/prophylactic surgery is life-saving in asymptomatic carriers, but clinical management remains challenging in carriers lacking clinical criteria. Through genotype-phenotype analysis in $\mathrm{P} /$ LP variant carrier-families, we studied $\mathrm{CDH} 1$-associated spectrum and age-of-disease onset to optimize clinical management. We collected/curated variant classification, family phenotypes and clinical-criteria of 506 coding and splice-site $C D H 1$-variant' carriers, registering 1361 phenotypes of 1302 family members from 10 European ERNGENTURIS countries. From 506 families, 160 (31.6\%) fulfilled HDGC clinical-criteria and $87.5 \%$ of these carried P/LP actionable variants (96.4\% truncating). From 346 (68.3\%) families lacking criteria, $13.3 \%$ carried such variants (100\% truncating). Among P/LP-variant bearing families fulfilling criteria, $48 \%$ of family phenotypes was DGC and $6 \%$ was LBC, both before 50 and without enrichment of other cancer types. In families lacking criteria, LBC increases to $24 \%$, and DGC drops to $8 \%$, still in the 40-50 age-range. $\mathrm{CDHl}$ missense variant carrierfamilies were almost completely excluded after clinical classification. Still, the ones remaining as P/LP revealed an enrichment in non-specified breast cancer and other cancer types, rarely presenting DGC and LBC. This is the first P/ LP $C D H 1$ variant-driven genotype-phenotype analysis. It demonstrates that clinically-actionable $C D H 1$ variants are mainly truncating, most carriers fulfill HDGC clinical- 
criteria, reinforcing use in clinical practice; supports 50 as the upper age-limit for $\mathrm{CDHI}$ testing; supports widening criteria to accommodate family history of LBC, and; highlight a likely irrelevant role of missense variants in HDGC.

J. García-Peláez: None. R. Monteiro: None. L. Sousa: None. H. Pinheiro: None. S. Castedo: None. L. Garrido: None. M. Teixeira: None. G. Michils: None. V. Bours: None. R. de Putter: None. L. Golmard \& M. Blanluet: None. C. Colas: None. P. Benusiglio: None. S. Aretz \& I. Spier: None. R. Hüneburg: None. L. Gieldon: None. E. Schröck: None. E. Holinski-Feder \& V. Steinke: None. D. Calistri \& G. Tedaldi: None. G. Ranzani: None. M. Genuardi: None. C. Silveira \& I. Silva: None. M. Krajc \& A. Blatnik: None. S. Novakovic: None. A. Patiño-García: None. J. Soto: None. C. Lázaro: None. G. Capellá: None. J. Brunet-Vidal: None. J. Balmaña: None. E. DomínguezGarrido: None. M. Ligtenberg: None. E. Fewings: None. R. Fitzgerald: None. E. Woodward: None. G. Evans: None. H. Hanson: None. K. Lagerstedt-Robinson: None. S. BajalicaLagercrantz: None. C. Egas: None. C. Martínez-Bouzas et al.: None. K. Dahan \& D. Feret: None. N. Hoogerbrugge: None. M. Tischkowitz: None. C. Oliveira: None.

\section{C10.4}

Enhancing rare variant interpretation in inherited arrhythmias through quantitative analysis of $\mathbf{5 1 8 2}$ cases from long QT syndrome and Brugada syndrome consortia cohorts and gnomAD population controls

\section{R. Walsh ${ }^{1 *}$, N. Lahrouchi ${ }^{l}$, C. Glinge ${ }^{1,2}$, C. Krijger ${ }^{l}, D$. Skoric-Milosavljevic ${ }^{l}$, N. Whiffin ${ }^{3}$, F. Mazzarotto ${ }^{4}$, J. S. Ware $^{3}$, R. Tadros ${ }^{5}$, International Brugada Syndrome Genetics Consortium, International LQTS Genetics Consortium, C. R. Bezzina ${ }^{l}$}

${ }^{1}$ Amsterdam UMC, Amsterdam, Netherlands, ${ }^{2}$ Copenhagen University Hospital, Copenhagen, Denmark, ${ }^{3}$ Imperial College, London, United Kingdom, ${ }^{4}$ University of Florence, Florence, Italy, ${ }^{5}$ Montreal Heart Institute, Montreal, QC, Canada

Introduction: Guidelines for variant interpretation in Mendelian disease set stringent criteria to report variants as (likely) pathogenic, prioritising control of false positive rate over test sensitivity and diagnostic yield, and require customisation for the specific genetic characteristics of genedisease dyads. Inherited arrhythmias like long QT syndrome (LQTS) and Brugada syndrome (BrS) are genetically heterogeneous, with missense variants constituting the preponderance of disease-causing variants. Evidence from family segregation or functional assays to confirm pathogenicity are often unavailable or impractical in clinical genetic testing, leading to high rates of variants of uncertain significance and false negative test results.

Materials and Methods: We compared rare variant frequencies from 1847 LQTS (KCNQ1,KCNH2,SCN5A) and $3335 \mathrm{BrS}$ (SCN5A) cases from the International LQTS/ BrS Genetics Consortia to population-specific gnomAD data. We propose disease-specific criteria for ACMG/AMP evidence classes - rarity (PM2/BS1 rules) and enrichment of individual (PS4) and domain-specific (PM1) variants in cases over controls.

Results: Rare $S C N 5 A$ variant prevalence differed between $\mathrm{BrS}$ cases with spontaneous $(28.7 \%)$ versus induced $(15.8 \%)$ type 1 Brugada ECG $\left(\mathrm{p}=1.3 \times 10^{-13}\right)$ and between European $(20.8 \%)$ and Japanese $(8.9 \%)$ patients $\left(\mathrm{p}=8.8 \times 10^{-18}\right)$. Transmembrane regions and specific $\mathrm{N}$ terminus $(\mathrm{KCNH} 2)$ and $\mathrm{C}$-terminus (KCNQ1/KCNH2) domains were characterised by high enrichment of case variants and $>95 \%$ probability of pathogenicity. Applying the customised rules, $17.5 \%$ of European $\mathrm{BrS}$ cases and $73.7 \%$ of European LQTS cases had variants classified as (likely) pathogenic, compared to estimated diagnostic yields (case excess over gnomAD) of 19.3\%/82.6\%.

Conclusions: Large case/control datasets enable quantitative implementation of ACMG/AMP guidelines and increased sensitivity for inherited arrhythmia genetic testing.

R. Walsh: None. N. Lahrouchi: None. C. Glinge: None. C. Krijger: None. D. Skoric-Milosavljevic: None. N. Whiffin: None. F. Mazzarotto: None. J. S. Ware: None. R. Tadros: None. C. R. Bezzina: None.

\section{C10.5}

Genome wide association study based on cardiac magnetic resonance imaging in 23,634 individuals identifies five new loci associated with left atrial enlargement

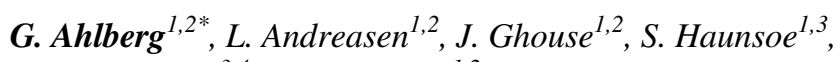
J. H. Svendsen ${ }^{3,4}$, M. S. Olesen ${ }^{1,2}$

${ }^{1}$ Laboratory for Molecular Cardiology, Department of Cardiology, Centre for Cardiac, Vascular, Pulmonary and Infectious Diseases, Rigshospitalet, University Hospital of Copenhagen, Copenhagen, Denmark, ${ }^{2}$ Department of Biomedical Sciences, University of Copenhagen, Copenhagen, Denmark, ${ }^{3}$ Department of Clinical Medicine, University of Copenhagen, Copenhagen, Denmark, ${ }^{4}$ Department of Cardiology, Centre for Cardiac, Vascular, Pulmonary and Infectious Diseases, Rigshospitalet, University Hospital of Copenhagen, Copenhagen, Denmark

Background: Left atrial enlargement is associated with mortality due to cardiovascular disease. The pathology behind and the association with cardiovascular outcomes 
are, however, poorly characterised. Compromised atrial function predisposes for stroke.

Methods: We obtained data from the UK Biobank cardiovascular magnetic resonance (CMR) protocol containing CMR scans from $\sim 31,000$ individuals at the time of study. Genome-wide association studies were carried out on state-of-the-art imaging variables exposed to machine learning algorithms to investigate the genetic basis of left atrial volume and function.

Results: We tested 10.6 million variants for association with left atrial (LA) minimum/maximum volumes and LA emptying fraction in 23,624 European individuals without prevalent myocardial infarction or heart failure. We identified five genetic loci located in close proximity to the genes EIF2D, C9orf3, ANKRD1, FGFR2, and MYO18B. We observed a notable genetic correlation between both maximum and minimum LA volumes and stroke and cardioembolic stroke.

Conclusions: We report on five genetic loci and suggest several plausible candidate genes important for atrial structural pathology. Genetic correlation analyses indicate that the genetic determinants of LA volumes could be markers of stroke risk. These findings enhance our understanding of the importance of the LA and are potential novel therapeutic targets for cardiovascular disease, including stroke.

G. Ahlberg: None. L. Andreasen: None. J. Ghouse: None. S. Haunsoe: None. J. H. Svendsen: None. M. S. Olesen: None.

\section{C10.6}

\section{Genetic interactions modulate lipid plasma levels and} cellular uptake

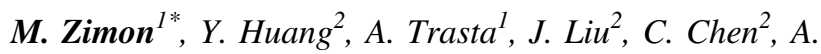

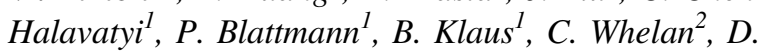
Sexton $^{2}$, S. John ${ }^{2}$, E. Tsai ${ }^{2}$, W. Huber ${ }^{l}$, R. Pepperkok ${ }^{l}, H$. Runz $^{2}$

${ }^{1}$ EMBL, Heidelberg, Germany, ${ }^{2}$ Biogen Inc., Cambridge, MA, United States

Introduction: Genetic Interactions (GIs), where variants in two genes collectively cause non-additive phenotypes different from the sum of the variants' individual effects, are considered a foundational element in the genetic architecture of complex traits. In humans, however, GIs have remained largely elusive. Here, we combined exomesequencing and genotyping in the UK Biobank with systematic cell-based RNA-interference (RNAi) to assess whether GIs between lipid genes contribute to changes in plasma lipid levels as risk factors for coronary artery disease (CAD).

Materials and Methods: We systematically tested for modifier effects between 30 lipid-regulatory genes derived from GWAS loci associated with plasma LDL, HDL, TG, TC or CAD using robust linear model fitting with three complementary data types: protein-truncating variants (PTVs) from whole-exome sequencing of 161,508 UK Biobank participants; GWAS lead SNPs from 378,033 UK Biobank participants; and results from a combinatorial RNAi screen measuring cellular LDL-uptake.

Results: We show that additive effects between lipid genes are pervasive, consistent with the use of summed effect sizes in polygenic risk scores. Yet, all three approaches also indicated existence of non-additive GIs, with one GI identified from PTV-based, 26 from SNPbased, and 20 from cell-based analyses, respectively. Integrating genetic and functional data validated three GIs as modulating both plasma lipid levels and cellular uptake.

Conclusions: Our combined genetic and functional analyses propose distinct gene pairs that modulate plasma and cellular lipid levels via additive and non-additive effects. Our results can help prioritize drug target pairs for the development of lipid-lowering combination therapies.

M. Zimon: None. Y. Huang: A. Employment (full or parttime); Significant; Biogen Inc. E. Ownership Interest (stock, stock options, patent or other intellectual property); Significant; Biogen Inc. A. Trasta: None. J. Liu: A. Employment (full or part-time); Significant; Biogen Inc. E. Ownership Interest (stock, stock options, patent or other intellectual property); Significant; Biogen Inc. C. Chen: A. Employment (full or part-time); Significant; Biogen Inc. E. Ownership Interest (stock, stock options, patent or other intellectual property); Significant; Biogen Inc. A. Halavatyi: None. P. Blattmann: None. B. Klaus: None. C. Whelan: A. Employment (full or part-time); Significant; Biogen Inc. E. Ownership Interest (stock, stock options, patent or other intellectual property); Significant; Biogen Inc. D. Sexton: A. Employment (full or part-time); Significant; Biogen Inc. E. Ownership Interest (stock, stock options, patent or other intellectual property); Significant; Biogen Inc. S. John: A. Employment (full or part-time); Significant; Biogen Inc. E. Ownership Interest (stock, stock options, patent or other intellectual property); Significant; Biogen Inc. E. Tsai: A. Employment (full or part-time); Significant; Biogen Inc. E. Ownership Interest (stock, stock options, patent or other intellectual property); Significant; Biogen Inc. W. Huber: None. R. Pepperkok: None. H. Runz: A. Employment (full or part-time); Significant; Biogen Inc. E. Ownership Interest (stock, stock options, patent or other intellectual property); Significant; Biogen Inc. 


\section{C11 Multiple Malformation Syndromes I}

\section{C11.1}

Biallelic variants in $K Y N U$ cause a multisystemic syndrome with hand hyperphalangism

N. Ehmke ${ }^{I^{*}}$, K. Cusmano-Ozog ${ }^{2}$, R. Koenig ${ }^{3}$, M. Holtgrewe $^{4,5}$, B. Nur ${ }^{6}$, E. Mihci ${ }^{6}$, H. Babcock ${ }^{7}$, C. GonzagaJauregui $^{8}$, J. D. Overton ${ }^{9}$, J. Xiao $^{10}$, B. Fischer-Zirnsak ${ }^{1}$, C. Huber $^{11}$, U. Kornak ${ }^{1}$, S. H. Elsea ${ }^{12}$, V. Cormier-Daire ${ }^{13}, C$. R. Ferreira ${ }^{14,15}$

${ }^{1}$ Institute of Medical Genetics and Human Genetics, Berlin, Germany, ${ }^{2}$ Biochemical Genetics and Metabolism Laboratory, Children's National Medical Center, Washington, DC, United States, ${ }^{3}$ Department of Human Genetics, University of Frankfurt, Frankfurt, Germany, ${ }^{4}$ Core Unit Bioinformatics - CUBI, Berlin Institute of Health, Berlin, Germany, ${ }^{5}$ Charité - Universitätsmedizin Berlin, Berlin, Germany, ${ }^{6}$ Department of Pediatric Genetics, Akdeniz University Medical School, Antalya, Turkey, ${ }^{7}$ Rare Disease Institute, Children's National Health System, Washington, DC, United States, ${ }^{8}$ Regeneron Genetics Center, Regeneron Pharmaceuticals Inc. Tarrytown, Tarrytown, NY, United States, ${ }^{9}$ Regeneron Genetics Center, Regeneron Pharmaceuticals Inc. TarrytownInstitute of Medical Genetics and Human Genetics, Tarrytown, NY, United States, ${ }^{10}$ Department of Molecular and Human Genetics, Baylor College of Medicine, Houston, TX, United States, ${ }^{11}$ Department of Genetics, INSERM UMR 1163, Université Paris DescartesSorbonne Paris Cité, Institut Imagine, AP-HP, Hôpital Necker Enfants MaladesInstitute of Medical Genetics and Human Genetics, Paris, France, ${ }^{12}$ Department of Molecular and Human Genetics, Baylor College of MedicineInstitute of Medical Genetics and Human Genetics, Houston, TX, United States, ${ }^{13}$ Department of Genetics, INSERM UMR 1163, Université Paris Descartes-Sorbonne Paris Cité, Institut Imagine, AP-HP, Hôpital Necker Enfants Malades, Paris, France, ${ }^{14}$ Institute of Medical Genetics and Human GeRare Disease Institute, Children's National Health Systemnetics, Washington, DC, United States, ${ }^{15}$ National Human Genome Research Institute, National Institutes of Health, Bethesda, MD, United States

Catel-Manzke syndrome is characterized by the combination of Pierre Robin sequence and radial deviation, shortening as well as clinodactyly of the index fingers, due to an accessory ossification center. Mutations in TGDS have been identified as one cause of Catel-Manzke syndrome, but cannot be found as causative in every patient with the clinical diagnosis. We performed a chromosome microarray and/or exome sequencing in three patients with hand hyperphalangism, heart defect, short stature, and mild to severe developmental delay, all of whom were initially given a clinical diagnosis of Catel-Manzke syndrome. In all patients, we detected biallelic variants in $K Y N U$, mainly missense variants. Plasma and urine metabolomic analysis of two patients indicated a block along the tryptophan catabolic pathway and urine organic acid analysis showed excretion of xanthurenic acid. Biallelic loss-of-function mutations in $K Y N U$ were recently described as a cause of NAD deficiency with vertebral, cardiac, renal and limb defects; however, no hand hyperphalangism and excretion of xanthurenic acid were described in those patients, and Catel-Manzke syndrome was not discussed as a differential diagnosis. In conclusion, we present unrelated patients identified with biallelic variants in $K Y N U$ leading to kynureninase deficiency and xanthurenic aciduria as a very likely cause of their hyperphalangism, heart defect, short stature, and developmental delay. We found urine organic acid analysis as a simple method that will facilitate differential diagnosis in patients with hyperphalangism and suspected Catel-Manzke syndrome. Funding resources: RahelHirsch program (Charité); Intramural Research Program, National Human Genome Research Institute, National Institutes of Health.

N. Ehmke: None. K. Cusmano-Ozog: None. R. Koenig: None. M. Holtgrewe: None. B. Nur: None. E. Mihci: None. H. Babcock: None. C. Gonzaga-Jauregui: A. Employment (full or part-time); Significant; Regeneron Genetics Center from Regeneron Pharmaceuticals Inc. J. D. Overton: None. J. Xiao: None. B. Fischer-Zirnsak: None. C. Huber: None. U. Kornak: None. S. H. Elsea: None. V. Cormier-Daire: None. C. R. Ferreira: None.

\section{C11.2}

Integrated genome and transcriptome analyses solves about one third of the patients with rare developmental disorders and negative first-line molecular investigations

A. Vitobello $^{1,2 *}$, F. Tran Mau-Them ${ }^{1,2}$, A. L. Bruel ${ }^{1,2}$, Y. Duffourd $^{l}$, E. Tisserant ${ }^{l}$, P. Callier ${ }^{1,2}$, S. Moutton ${ }^{1,3}, S$. Nambot $^{1,3}$, D. Lehalle ${ }^{1,3}$, N. Jean-Marçais ${ }^{3}$, J. Delanne ${ }^{1,3}$,

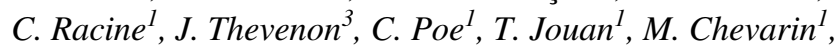
M. Willems ${ }^{4}$, C. Coubes $^{4}$, D. Geneviève ${ }^{4,5}$, N. Houcinat ${ }^{1,3}$, A. Masurel-Paulet ${ }^{3}$, A. Mosca-Boidron ${ }^{1,2}$, A. Sorlin ${ }^{1,2,3}$, B. Isidor $^{6}$, S. Heide ${ }^{7}$, A. Afenjar ${ }^{8}$, D. Rodriguez ${ }^{9}$, C. Mignot $^{7}$, D. Heron ${ }^{7}$, M. Vincent ${ }^{6}$, P. Charles ${ }^{7}, S$. Odent $^{10}, C$.

Dubourg ${ }^{11}$, A. Faudet ${ }^{7}$, B. Keren ${ }^{7}$, B. Cognél ${ }^{12}$, A. Boland ${ }^{13}$, R. Olaso ${ }^{13}$, C. Philippe P., $^{1,}$ J. F. Deleuze ${ }^{13}$, L. Faivre F $^{1,3}, C$. Thauvin-Robinet ${ }^{1,2,3}$

${ }^{1}$ Inserm UMR 1231 GAD team, Genetics of Developmental disorders, Université de Bourgogne-Franche Comté, Dijon, France, ${ }^{2}$ Unité Fonctionnelle d'Innovation diagnostique des maladies rares, Pôle de Biologie, CHU Dijon 
Bourgogne, Dijon, France, ${ }^{3}$ Centre de Référence maladies rares "Anomalies du Développement et syndrome malformatifs » de l'Est, Hôpital d'Enfants, CHU Dijon Bourgogne, Dijon, France, ${ }^{4}$ Département de Génétique Médicale, Maladies Rares et Médecine Personnalisée, Centre de Référence Maladies Rares Anomalies du Développement et Syndromes Malformatifs ADSOOR, CHU Montpellier, Montpellier, France, ${ }^{5}$ Université Montpellier, Unité Inserm U1183, Montpellier, France, ${ }^{6} \mathrm{CHU}$ de Nantes, Service de Génétique Médicale, Nantes, France, ${ }^{7}$ AP-HP, Département de Génétique, Hôpital de la PitiéSalpêtrière, 75013, Paris, France et Centre de Référence "déficiences intellectuelles de causes rares", 75013, Paris, France, ${ }^{8}$ APHP, Département de génétique et embryologie médicale, Hôpital Armand Trousseau, F-75012, Paris, France, ${ }^{9}$ Centre de Référence de Neurogénétique et Service de Neurologie Pédiatrique, AP-HP, Hôpital Armand Trousseau, GHUEP ; Sorbonne Université, GRC $n^{\circ} 19$, pathologies Congénitales du Cervelet-LeucoDystrophies, Paris, France, ${ }^{10} \mathrm{CHU}$ de RENNES, Génétique; Univ Rennes, CNRS, IGDR (Institut de Génétique et Développement de Rennes) UMR 6290, Rennes, France, ${ }^{11}$ Service de Génétique Moléculaire et Génomique, BMT-HC «Jean Dausset ", CHU Pontchaillou, 2 rue Henri Le Guilloux, 35033, Rennes, France, ${ }^{12}$ L'institut du thorax, INSERM, CNRS, UNIV Nantes, CHU de Nantes, Nantes, France., Nantes, France, ${ }^{13}$ Centre National de Recherche en Génomique Humaine (CNRGH), Institut de Biologie François Jacob, CEA, Université Paris-Saclay and LabEx GENMED, F-91057, Evry, France

Exome sequencing (ES) represents the first-tier diagnostic test in patients presenting with syndromic developmental delay with suspected monogenic etiology. Yet, about 50\% of these patients remain unsolved, arguing the interest to extend the genetic investigations beyond the protein-coding genome and to integrate multi-omics approaches. We launched a multi-centric pilot study gathering 53 unsolved patients, after trio ES and array-CGH results, presenting with heterogeneous mild to severe syndromic intellectual disabilities. We performed genome sequencing (GS) combined with transcriptome analysis, highlighting a molecular cause in $32 \%$ of the cohort (18/53 patients). GS identified 7 causative structural variants, including 1 deletion, 2 balanced inversions, 1 balanced translocation and 3 complex variants. The molecular readouts of such variants were all validated and furtherly investigated by RNAseq. One deep intronic SNV causing the activation of a cryptic exon, changing the open reading frame of the transcript, was detected by RNAseq. Two frameshift-causing indels were identified in protein-coding regions not captured by ES. Three variants were identified in genes not yet known as disease-causing at the time of the ES analysis. Finally, genotype-phenotype correlation could establish 3 additional diagnoses not identified during the ES analysis. In addition, we detected 2 complex structural variants of unknown significance, not resolvable by short-read GS, as well as new candidate genes identified through RNA-seq differential expression analysis. Overall, GS and RNAseq analyses allowed the identification of the molecular mechanisms underlying $1 / 3$ of previously unsolved patients, and additional candidate variants requiring further investigation $\left(3^{\text {rd }}\right.$-generation sequencing, $3 \mathrm{C}$ techniques $)$ to demonstrate their causality.

A. Vitobello: None. F. Tran Mau-Them: None. A. L. Bruel: None. Y. Duffourd: None. E. Tisserant: None. P. Callier: None. S. Moutton: None. S. Nambot: None. D. Lehalle: None. N. Jean-Marçais: None. J. Delanne: None. C. Racine: None. J. Thevenon: None. C. Poe: None. T. Jouan: None. M. Chevarin: None. M. Willems: None. C. Coubes: None. D. Geneviève: None. N. Houcinat: None. A. Masurel-Paulet: None. A. Mosca-Boidron: None. A. Sorlin: None. B. Isidor: None. S. Heide: None. A. Afenjar: None. D. Rodriguez: None. C. Mignot: None. D. Heron: None. M. Vincent: None. P. Charles: None. S. Odent: None. C. Dubourg: None. A. Faudet: None. B. Keren: None. B. Cogné: None. A. Boland: None. R. Olaso: None. C. Philippe: None. J. F. Deleuze: None. L. Faivre: None. C. Thauvin-Robinet: None.

\section{C11.3}

Characterization and treatment of overactive $K_{A T P}$ channels associated with Cantú syndrome in zebrafish

H. I. Roessler ${ }^{1 *}$, S. S. Singareddy ${ }^{2}$, C. McClenaghan ${ }^{2}, S$. Savelberg' ${ }^{1}$, F. Tessadori ${ }^{3}, J$. Bakkers $^{3}, R$. Tyron $^{2}, C . G$. Nichols $^{2}$, G. van Haaften ${ }^{1}$

${ }^{1}$ Department of Genetics, Center for Molecular Medicine, University Medical Center Utrecht, Utrecht, Netherlands, ${ }^{2}$ Department of Cell Biology and Physiology, and Center for the Investigation of Membrane Excitability Diseases (CIMED), Washington University, St. Louis, MO, United States, ${ }^{3}$ Hubrecht Institute-KNAW and University Medical Centre Utrecht, Utrecht, Netherlands

Cantú Syndrome (CS) is a rare genetic disorder caused by gain-of-function $(\mathrm{GoF})$ mutations in genes encoding the pore-forming (Kir6.1, KCNJ8) and regulatory (SUR2, $A B C C 9)$ subunits of cardiovascular ATP-sensitive potassium $\left(\mathrm{K}_{\mathrm{ATP}}\right)$ channels. CS patients consistently present with hypertrichosis and facial anomalies alongside a varying range of cardiovascular abnormalities. CS is debilitating with no targeted therapy available. To further elucidate the complex pathophysiology of CS and to perform therapeutic drug screening, we introduced patient-specific point 
mutations in the germline of zebrafish orthologues of $A B C C 9$ and KCNJ8. Applying RT-PCR and patch-clamping of isolated ventricular cardiomyocytes (VCMs) and vascular smooth muscle cells (VSMCs) we confirm for the first time that expression, composition and functional properties of cardiovascular $\mathrm{K}_{\mathrm{ATP}}$ channels are conserved between zebrafish and mammals. CS-related GoF in mutant fish was validated and is reflected by a shift in ATP sensitivity in VCMs and a greater basal $\mathrm{K}$ conductance in VSMCs. It is postulated that by directly targeting overactive $\mathrm{K}_{\mathrm{ATP}}$ channels repurposing channel blockers, it may be possible to reverse consequences of GoF. Thus, we tested the in vivo efficacy of glibenclamide and repaglinide, clinically applied to block $\mathrm{GoF} \mathrm{K}_{\mathrm{ATP}}$ channels involved in neonatal diabetes, and the Kir6.1 inhibitor PNU-37883. Glibenclamide resulted in significant correction of CS cardiac phenotypes in $a b c c 9$ knock-in (KI) larvae, whereas repaglinide revealed similar effects in kcnj8 KI mutants. These results indicate that zebrafish represent a suitable model to study pathophysiology and therapy options for CS and further disorders involving aberrant cardiovascular $\mathrm{K}_{\mathrm{ATP}}$ channels.

E-Rare 2014 'CantuTreat' (I-2101-B26) to GvH.

H. I. Roessler: None. S. S. Singareddy: None. C. McClenaghan: None. S. Savelberg: None. F. Tessadori: None. J. Bakkers: None. R. Tyron: None. C. G. Nichols: None. G. van Haaften: None.

\section{C11.4}

Biallelic MADD variants cause a phenotypic spectrum ranging from developmental delay to a multisystem disorder

P. E. Schneeberger ${ }^{*}$, F. Kortüm ${ }^{l}$, G. C. Korenke $^{2}, M$.

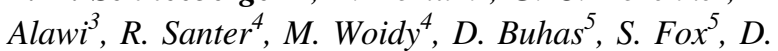
McKnight ${ }^{6}$, M. Alfadhel ${ }^{7}$, B. D. Webb ${ }^{8}$, E. G. Coci, R. Abou Jamra ${ }^{10}$, A. Finck ${ }^{10}$, M. Siekmeyer ${ }^{11}$, S. Biskup ${ }^{12}$, C. Heller $^{12}$, E. M. Maier ${ }^{13}$, P. Javaher-Haghighi ${ }^{14}$, M. F. Bedeschil $^{15}$, P. F. Ajmone ${ }^{16}$, M. Iascone ${ }^{17}$, H. Peeters ${ }^{18}, K$. Ballon $^{19}$, J. Jaeken ${ }^{20}$, A. Rodríguez Alonso ${ }^{21}$, M. Palomares-Bralo ${ }^{22}$, F. Santos-Simarro ${ }^{22}$, M. E. C.

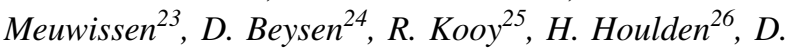
Murphy $^{26}$, M. Doosti ${ }^{27}$, E. G. Karimiani ${ }^{27}$, M. Mojarrad ${ }^{28}$, R. Maroofian ${ }^{26}$, B. D. Gelb ${ }^{8}$, I. Kurth ${ }^{29}$, M. Hempel $^{l}, K$. Kutsche $^{l}$

${ }^{1}$ Institute of Human Genetics, University Medical Center Hamburg-Eppendorf, Hamburg, Germany, ${ }^{2}$ Klinik für Neuropädiatrie und angeborene Stoffwechselerkrankungen, Klinikum Oldenburg, Oldenburg, Germany, ${ }^{3}$ Bioinformatics Core Unit, University Medical Center HamburgEppendorf, Hamburg, Germany, ${ }^{4}$ Department of Pediatrics, University Medical Center Hamburg-Eppendorf, Hamburg,
Germany, ${ }^{5}$ Division of Medical Genetics, Department of Specialized Medicine, McGill University Health Centre, Montreal, QC, Canada, ${ }^{6}$ GeneDx, Gaithersburg, MD, United States, ${ }^{7}$ Division of Genetics, Department of Pediatrics, King Abdullah specialized Children's Hospital, Riyadh, Saudi Arabia, ${ }^{8}$ Department of Genetics and Genomic Sciences, Icahn School of Medicine at Mount Sinai, New York, NY, United States, ${ }^{9}$ Department for Neuropediatrics, University Children's Hospital, Ruhr University Bochum, Bochum, Germany, ${ }^{10}$ Institute of Human Genetics, University Medical Center Leipzig, Leipzig, Germany, ${ }^{11}$ Universitätsklinikum Leipzig - AöR, University of Leipzig, Hospital for Children and Adolescents, Leipzig, Germany, ${ }^{12} \mathrm{CeGaT} \mathrm{GmbH}$ and Praxis für Humangenetik Tübingen, Tübingen, Germany, ${ }^{13} \mathrm{Dr}$. von Hauner Children's Hospital, University of Munich, Munich, Germany, ${ }^{14}$ Medicover Humangenetik Hannover, Hanover, Germany, ${ }^{15}$ Medical Genetic Unit, Fondazione IRCCS Ca' Granda Ospedale Maggiore Policlinico, Milan, Italy, ${ }^{16}$ Child and Adolescent Neuropsychiatric Unit, Fondazione IRCCS Cà Granda Ospedale Maggiore Policlinico, Milan, Italy, ${ }^{17}$ Laboratorio di Genetica Medica, ASST Papa Giovanni XXIII, Bergamo, Italy, ${ }^{18}$ Center for Human Genetics, KU Leuven, Leuven, Belgium, ${ }^{19}$ Centre for Developmental Disabilities, Department of Development and Regeneration, KU Leuven, Leuven, Belgium, ${ }^{20}$ Center for Metabolic Diseases, KU Leuven, Leuven, Belgium, ${ }^{21}$ Unidad de Patología Compleja, Servicio de Pediatría, Hospital Universitario La Paz, Madrid, Spain, ${ }^{22}$ Instituto de Genética Médica y Molecular, Hospital Universitario La Paz, IdiPAZ, CIBERER, ISCIII, Madrid, Spain, ${ }^{23}$ Center of Medical Genetics, University Hospital Antwerp, Antwerp, Belgium, ${ }^{24}$ Department of Pediatric Neurology, University Hospital Antwerp, Antwerp, Belgium, ${ }^{25}$ Department of Medical Genetics, University of Antwerp, Antwerp, Belgium, ${ }^{26}$ Department of Neuromuscular Diseases, UCL Queen Square Institute of Neurology and The National Hospital for Neurology and Neurosurgery, London, United Kingdom, ${ }^{27}$ Next Generation Genetic Polyclinic, Mashhad, Iran, Islamic Republic of, ${ }^{28}$ Department of Medical Genetics, Mashhad University of Medical Sciences, Mashhad, Iran, Islamic Republic of, ${ }^{29}$ Institute of Human Genetics, RWTH Aachen University, Aachen, Germany

We report a new pleiotropic disorder with a unique constellation of neurological, endocrine, exocrine, and hematological findings that is caused by biallelic $M A D D$ variants. MADD, the mitogen-activated protein kinase (MAPK) activating death domain protein, regulates various cellular functions, such as vesicle trafficking, activity of the Rab3 and Rab27 small GTPases, tumor necrosis factor- $\alpha$ (TNF$\alpha$ )-induced signaling and prevention of cell death. Through international collaboration, we collected 20 patients with 18 
different pathogenic $M A D D$ variants identified by nextgeneration sequencing. We clinically evaluated the patients and categorized the phenotypes in two groups. Eleven patients in Group 1 had severe developmental delay, endocrine and exocrine dysfunction, impairment of sensory and autonomic nervous system, and hematological anomalies. Nine patients in Group 2 presented with predominant neurological phenotype comprising developmental delay, hypotonia, speech impairment, and seizures. Relative quantification of MADD mRNA and protein in patients' fibroblasts showed drastic reduction or loss of MADD. Treatment of patient-derived fibroblasts with TNF- $\alpha$ resulted in reduced phosphorylation of ERK1/2, enhanced activation of the pro-apoptotic caspase- 3 and -7 and increased apoptosis compared to control cells. Internalization study of epidermal growth factor (EGF) in patient cells revealed a defect in endocytosis of EGF. We conclude that MADD deficiency underlies multiple cellular defects that can be attributed to alterations of TNF- $\alpha$-dependent signaling pathways and defects in vesicular trafficking. Our data highlights the multifaceted role of MADD as signaling molecule in different organs and reveals its physiological role in regulating the function of the sensory and autonomic nervous system and endocrine and exocrine glands.

P. E. Schneeberger: None. F. Kortüm: None. G. C. Korenke: None. M. Alawi: None. R. Santer: None. M. Woidy: None. D. Buhas: None. S. Fox: None. D. McKnight: None. M. Alfadhel: None. B. D. Webb: None. E. G. Coci: None. R. Abou Jamra: None. A. Finck: None. M. Siekmeyer: None. S. Biskup: None. C. Heller: None. E. M. Maier: None. P. Javaher-Haghighi: None. M. F. Bedeschi: None. P. F. Ajmone: None. M. Iascone: None. H. Peeters: None. K. Ballon: None. J. Jaeken: None. A. Rodríguez Alonso: None. M. Palomares-Bralo: None. F. Santos-Simarro: None. M. E. C. Meuwissen: None. D. Beysen: None. R. Kooy: None. H. Houlden: None. D. Murphy: None. M. Doosti: None. E. G. Karimiani: None. M. Mojarrad: None. R. Maroofian: None. B. D. Gelb: None. I. Kurth: None. M. Hempel: None. K. Kutsche: None.

\section{C11.5}

Modelling the developmental spliceosomal craniofacial disorder Burn-McKeown Syndrome using induced pluripotent stem cells

\section{K. A. Wood", C. Rowlands, H. Thomas, S. Woods, J. O'Flaherty, S. Kimber, W. Newman, R. O'Keefe}

\section{University of Manchester, Manchester, United Kingdom}

The developmental craniofacial disorder Burn-McKeown Syndrome (BMKS) is caused by compound heterozygous mutations in the pre-messenger RNA splicing factor
TXNL4A/DIB1. Patients with BMKS have a 34 base pair deletion in the promoter region of one allele of TXNL4A combined with a loss-of-function mutation in the other allele, resulting in reduced TXNLAA expression. However, it is unclear how reduced expression of this spliceosome protein results in craniofacial defects during development. Here we reprogramme BMKS patient and parent blood cells into induced pluripotent stem cells (iPSCs) and differentiate the iPSCs into induced neural crest cells (iNCCs). We find that patient-derived iPSCs proliferate more slowly than parent- and unrelated control-derived iPSCs. Patient iPSCs show defective differentiation into iNCCs compared to parent and unrelated control iPSCs, in particular undergoing an epithelial-to-mesenchymal transition. RNA-seq analysis of the iNCCs reveals considerable differential gene expression and mis-splicing in genes relevant to craniofacial development. The mis-spliced genes share common physical properties such as length and splice site strengths, suggesting that splicing of certain subsets of genes is particularly sensitive to changes in TXNL4A expression. We are comparing mis-expressed and mis-spliced genes in iPSCs and iNCCs from patients with parent and unrelated control lines to delineate why neural crest cells are particularly affected by reduced TXNL4A expression. Using this disease-relevant model, we will study properties such as migration and the cell cycle in patient-specific iNCCs to unravel how BMKS-specific mutations in TXNLAA result in craniofacial defects.

Grant references: $M R C$ (1916606; 1926882), BBSRC (BB/N000358/1), NIHR Manchester Biomedical Research Centre (IS-BRC-1215-20007).

K. A. Wood: None. C. Rowlands: None. H. Thomas: None. S. Woods: None. J. O'Flaherty: None. S. Kimber: None. W. Newman: None. R. O'Keefe: None.

\section{C11.6}

A novel Bannayan-Riley-Ruvalcaba (BRRS)/CLOVES syndrome model in Xenopus tropicalis, by CRISPR/ Cas9

\section{Dimitrakopoulou*, S. Demuynck, K. Vleminckx}

\section{Ghent Univeristy, Ghent, Belgium}

Introduction: Xenopus has been widely used in disciplines of developmental biology and functional genomics and the CRISPR/Cas9 breakthrough has significantly expanded its use for human disease modeling. Activating mutations in the PIK3CA gene and PTEN loss of function mutations generate PIK3CA-related overgrowth syndromes (PROS/ CLOVES) and Bannayan-Riley-Ruvalcaba (BRRS) syndrome, respectively. Although the genetic mutations that drive these syndromes are different, disease phenotypes 
overlap. Here we describe a BRRS/CLOVES model by CRISPR/Cas9 targeting of the pten gene in X. tropicalis.

Materials and methods: X.tropicalis 4-cell stage embryos were injected with pten $+m y c+n o t c h 1 *$ and pten + notchl ${ }^{*}$ sgRNA complexed with Cas9. After 5 weeks, froglets' limbs and main body demonstrated overgrowths and at critical point, animals were sacrificed. Overgrowths and internal organs were histologically processed. The mutation pattern of overgrowths was evaluated by targeted deep sequencing.

Results: The pten mutant animals, that presented overgrowths were dissected. These animals demonstrated muscle hyperthrophy and abdominal haemorrhage. Histological analysis characterized the overgrowths as lipomatous tumors. Furthermore, immunohistochemical analysis of lipomas demonstrated highly activated PI3K/AKT pathway. Internal organs displayed disorganized architecture and highly activated PI3K/AKT pathway. Samples from overgrowths were sequenced, revealing that bi-allelic inactivation of the pten gene is sufficient for CLOVES development. Pten mutants will be used for validating our model by testing BYL719, a PIK3CA inhibitor with efficacy in treating human CLOVES patients.

Conclusion: We generated the first amphibian model in BRRS/CLOVES syndrome. We believe that a Xenopus tropicalis BRRS/CLOVES model could become a valid experimental platform for further compound testing or identification of new drug targets.

D. Dimitrakopoulou: None. S. Demuynck: None. K. Vleminckx: None.

\section{C12 Neuropsychiatry}

\section{C12.1}

Polygenic background of psychotic disorders and genetic determinants of disease severity

A. V. Ahola-Olli ${ }^{*}$, Z. Misiewicz ${ }^{2}, N$. Mars $^{2}$, M. Lähteenvuo $^{3}$, B. Neale ${ }^{1}$, T. Männynsalo ${ }^{4}$, E. Isometsä ${ }^{5}, A$. Wegelius $^{5}$, J. Hietala ${ }^{6}$, W. Haaki ${ }^{6}$, O. Kampman ${ }^{7}$, J. Veijola $^{8}$, J. Tiihonen ${ }^{3}$, T. Kieseppä ${ }^{9}$, J. Suvisaari ${ }^{9}, S$. Hyman $^{1}$, M. Daly ${ }^{2}$, A. Palotie ${ }^{2}$

${ }^{1}$ Broad Institute of MIT and Harvard, Cambridge, MA, United States, ${ }^{2}$ Institute for Molecular Medicine Finland (FIMM), Helsinki, Finland, ${ }^{3}$ Department of Forensic Psychiatry, University of Eastern Finland, Niuvanniemi Hospital, Kuopio, Finland, ${ }^{4}$ Department of Psychiatry, Helsinki City Health Centre, Helsinki, Finland, ${ }^{5}$ Department of Psychiatry, University of Helsinki and Helsinki University Hospital, Helsinki, Finland, ${ }^{6}$ Department of Psychiatry, Turku University Hospital, Turku, Finland, ${ }^{7}$ Faculty of Medicine and Life Sciences, Tampere
University, Tampere, Finland, ${ }^{8}$ Department of Psychiatry, Research Unit of Clinical Neuroscience, University of Oulu, Oulu, Finland, ${ }^{9}$ Department of Public Health Solutions, Mental Health Unit, National Institute for Health and Welfare, Helsinki, Finland

Introduction: SUPER-Finland is a nationwide study which has recruited 10,075 patients with psychotic disorder across the country. Because of comprehensive national registers, longitudinal data on education, employment, hospitalizations, drug prescriptions and cause of death can be drawn from registers and linked to information collected at study visit using national social security number. Our aim is to shed light to genetic basis of different psychotic disorders.

Materials and Methods: Illumina Global Screening Array was used to genotype 10,075 subjects from SUPERFinland study. Genotypes were imputed using populationspecific Sequencing Initiative Suomi panel as reference. Polygenic risk scores were calculated with LDpred software using publicly available genome-wide association study summary statistics. Data on hospitalizations, duration of involuntary hospitalization and psychosocial functioning at hospitalization and discharge was drawn from registers.

Results: When comparing PRSs for schizophrenia and major depression jointly, subjects with schizoaffective disorder have different polygenic background than those with schizophrenia and bipolar disorder. Schizoaffective disorder is also different from psychotic depression. Subjects with high polygenic risk for schizophrenia have lower psychosocial functioning at hospital discharge, possibly reflecting residual disease. They also have more involuntary hospital days.

Conclusions: Schizoaffective disorder seems to have different polygenic background from other forms of psychotic illnesses. This supports the idea of maintaining it as separate disorder in psychiatric disease classification. High polygenic risk for psychotic illness predisposes to more severe disease forms.

Funding: The Stanley Center for Psychiatric Research at the Broad Institute of MIT and Harvard, Orion Research Foundation, Finnish Cultural Foundation.

A. V. Ahola-Olli: None. Z. Misiewicz: None. N. Mars: None. M. Lähteenvuo: None. B. Neale: None. T. Männynsalo: None. E. Isometsä: None. A. Wegelius: None. J. Hietala: None. W. Haaki: None. O. Kampman: None. J. Veijola: None. J. Tiihonen: F. Consultant/Advisory Board; Modest; Lundbeck. Other; Modest; Eli Lilly, Janssen-Cilag. T. Kieseppä: None. J. Suvisaari: None. S. Hyman: None. M. Daly: None. A. Palotie: None.

\section{C12.2}

Autism-linked Cullin3 germline haploinsufficiency impacts cytoskeletal dynamics and cortical neurogenesis through RhoA signaling 
M. Amar ${ }^{l}$, A. Pramod ${ }^{l}$, V. Munive Herrera ${ }^{l}, N . Y^{2}$, L. R. Qiu $^{3}$, P. Moran-Losada ${ }^{l}$, P. Zhang ${ }^{l}$, C. A. Trujillo ${ }^{l}, J$. Ellegood $^{3}$, J. Urresti ${ }^{1}$, K. Chau ${ }^{l}, J$. Diedrich ${ }^{2}, J$. Chen $^{l}, J$. Gutierrez $^{l}$, J. Sebat ${ }^{1}$, D. Ramanathan ${ }^{1}, J$. P. Lerch ${ }^{3}, J . R$. Yates III $^{2}$, A. R. Muotri ${ }^{l}$, L. M. Iakoucheva ${ }^{l *}$

${ }^{1}$ University of California San Diego, La Jolla, CA, United States, ${ }^{2}$ The Scripps Research Institute, La Jolla, CA, United States, ${ }^{3}$ Hospital for Sick Children, Toronto, ON, Canada

Introduction: E3-ubiquitin ligase Cullin3 (Cul3) is a high confidence risk gene for Neurodevelopmental Disorders (NDD) including Autism Spectrum Disorder (ASD) and Developmental Delay (DD). To better understand the impact of ASD-associated Cul3 mutations on brain development, we generated Cul3-haploinsufficient mouse model using CRISPR/Cas9 genome editing technology.

Materials and Methods: We investigated brain anatomy, behavior, molecular, cellular, and circuit-level mechanisms dysregulated by $\mathrm{Cul3}$ mutations. Brain MRI, transcriptomic and quantitative proteomic profiling of cerebral cortex, hippocampus, and cerebellum of Cul3-haploinsufficient mice were carried out at three developmental periods (embryonic, early postnatal and adult) to understand spatiotemporal impact of $\mathrm{Cul3}$ mutations.

Results: Brain MRI of $\mathrm{Cul3}$ mutant animals found profound abnormalities in half of brain regions, including decreased volume of cortical regions and increased volume of subcortical regions. Cul3 mutant mice exhibited social and cognitive deficits, and hyperactive behavior. Spatiotemporal transcriptomic and proteomic profiling of the brain implicated neurogenesis and cytoskeletal defects as key drivers of $\mathrm{Cul3}$ functional impact. Cortical neurons from mutant mice had reduced dendritic length and loss of filamentous actin puncta, along with reduced spontaneous network activity. Inhibition of small GTPase RhoA, a molecular substrate of Cul3 ligase, rescued dendrite length phenotype.

Conclusions: Novel CRISPR/Cas9 Cul3-haploinsufficient mouse model implicated dysregulation of RhoA pathway and downstream cytoskeletal and neurogenesis defects during early brain development in autism. This study identified RhoA signaling as a potential mechanism, through which $\mathrm{Cul3}$ mutation impacts early brain development.

Funding: NIH grants MH108528, MH109885, MH105524, MH104766, and Simons Foundation for Autism Research \#345469.

M. Amar: None. A. Pramod: None. V. Munive Herrera: None. N. Yu: None. L. R. Qiu: None. P. Moran-Losada: None. P. Zhang: None. C. A. Trujillo: None. J. Ellegood: None. J. Urresti: None. K. Chau: None. J. Diedrich: None.
J. Chen: None. J. Gutierrez: None. J. Sebat: None. D. Ramanathan: None. J. P. Lerch: None. J. R. Yates III: None. A. R. Muotri: None. L. M. Iakoucheva: None.

\section{C12.3}

Tissue-specific transcriptional and functional signatures in reciprocal genomic disorders : Insights from integrated mouse and human neuronal models

R. Yadav ${ }^{1,2,3^{*}}$, D. JC Tai ${ }^{1,2,3}, J$. Wang ${ }^{4,5}$, T. Aneichyk ${ }^{1,2}, S$. Erdin $^{1,3}$, B. B Currall ${ }^{1,2,3}$, K. O'Keefe ${ }^{1,2}$, A. Stortchevoi ${ }^{1,2}$, U. Rudolph ${ }^{6}$, R. H. Perlis ${ }^{4,5}$, J. F Gusella, ${ }^{1,2,3,7}$, M. E Talkowskil ${ }^{1,2,3}$

${ }^{I}$ Center for Genomic Medicine and Department of Neurology, Massachusetts General Hospital, Boston, MA, United States, ${ }^{2}$ Department of Neurology, Harvard Medical School, Boston, MA, United States, ${ }^{3}$ Program in Medical and Population Genetics, Broad Institute of MIT and Harvard, Cambridge, MA, United States, ${ }^{4}$ Center for Quantitative Health, Center for Genomic Medicine, and Department of Psychiatry, Massachusetts General Hospital, Boston, MA, United States, ${ }^{5}$ Department of Psychiatry, Harvard Medical School, Boston, MA, United States, ${ }^{6} 7$ Department of Comparative Biosciences, College of Veterinary Medicine, University of Illinois at UrbanaChampaign, Urbana, IL, United States, ${ }^{7}$ Department of Genetics, Harvard Medical School, Boton, MA, United States

Reciprocal genomic disorders (RGDs) involve large copy number variation $(\mathrm{CNV})$ mediated by flanking segmental duplications. Though individually rare, the collective contribution of RGDs to developmental disorders is substantial. Here, we compared the tissue-specific functional and transcriptional signatures associated with two RGDs: 16p11.2 (740 kb, 29 genes) and 15q13.3 (1.5 Mb, 7 genes). For $16 \mathrm{p} 11.2$ we generated 304 RNAseq libraries across three tissues cerebellum, prefrontal cortex, and striatum from 101 total mice, and isogenic CRISPR-engineered human neural stem cells (NSCs, $n=27$ lines) and NGN2-induced neurons (iNs, $\mathrm{n}=19$ lines), while 119 libraries were analyzed for 15q13.3 across NSCs (24 lines), iNs (23 lines), and brain tissue from 48 mice. Transcriptome profiling of both the RGDs revealed that the strongest and most consistent effects occurred within the CNV segment while most secondary regulatory effects were tissue-specific. Co-expression analyses isolated modules that were enriched for RGD genes, DEGs, and highly constrained and genes associated with NDD and autism. Genes regulating actin filament and cytoskeleton organization, neuron projection, calcium signaling pathway, and ion channel activity were significantly altered in $15 \mathrm{q} 13.3$ iNs. Using a weighted z-score meta- 
analysis, we discovered convergence on shared molecular pathways across the RGDs, most prominently including alterations to genes involved in myelin sheath formation, dendritic function, and mitochondrial function. Intriguingly, neuron phenotyping assays revealed significant antagonistic effect of these RGDs on neurite length and number of branchpoints in iNs. These results suggest the pathogenic mechanisms associated with recurrent RGDs may converge on common molecular signatures.

R. Yadav: None. D. JC Tai: None. J. Wang: None. T. Aneichyk: None. S. Erdin: None. B. B Currall: None. K. O'Keefe: None. A. Stortchevoi: None. U. Rudolph: None. R. H. Perlis: None. J. F Gusella: None. M. E Talkowski: None.

\section{C12.4}

Autism Comorbidities: Role of $\mathrm{CHD} 8$ during the Development of the Enteric Nervous System

\section{G. L. Hayot ${ }^{*}$, C. Weber, C. Golzio \\ IGBMC, Illkirch-Graffenstaden, France}

Introduction: $C H D 8$ is mutated in $0.21 \%$ of total autism spectrum disorders (ASD) cases. Recently, clinical reassessment highlighted the high prevalence of gastrointestinal (GI) defects in ASD. Transient suppression of $\operatorname{chd} 8$ in zebrafish led to a reduced number of enteric neurons and a decreased gut mobility which likely drive the GI problems (Bernier et al. Cell, 2014).

Materials and Methods: To understand how the loss of chd8 causes the loss of mature enteric neurons, we generated and characterized a stable mutant zebrafish line: $T g\left(\right.$ chd $8^{\text {sal9827}}$;phox $\left.2 b b: G F P\right)$ in which the neuronal progenitors (i.e. enteric neural crest cells, eNCCs) are labeled with GFP.

Results: First, we observed a migration delay and reduced proliferation of eNCCs during early development and a decreased number of mature neurons and more specifically serotonergic neurons at 5 days post-fertilization. Second, transcriptomic analyses of eNCCs revealed the upregulation of the serotonin receptor htr6 and the dysregulation of several actors of the Wnt/Planar Cell Polarity and RhoA pathways, extracellular matrix components, and several genes from the human morbid genome. Third, behavioral analyses showed $i$ ) hyperactivity of the mutant chd8 larvae and ii) normal sociability and no aggressive behavior in the adult mutants.

Conclusion: We showed that the decreased number of enteric neurons in absence of $c h d 8$ is due to a combination of proliferation, migration and differentiation defects of the eNCCs. Moreover, our transcriptomic data identified druggable targets in $c h d 8$-associated signaling pathways and we are currently performing rescue experiments with small molecules in the chd8 mutants.

Grants: ANR-10-IDEX-0002-02, ANR-10-LABX-0030INRT

G. L. Hayot: None. C. Weber: None. C. Golzio: None.

\section{C12.5}

A human single-cell atlas of the Substantia nigra reveals novel cell-specific pathways associated with the genetic risk of Parkinson's disease and neuropsychiatric disorders.

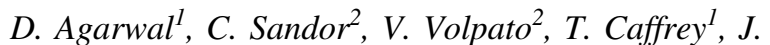
Monzon-Sandoval $^{2}, R$. Bowden ${ }^{l}, J$. Alegre-Abarrategui ${ }^{1}, R$. Wade-Martins $^{l}$, C. Webber $^{2, l^{*}}$

${ }^{1}$ Oxford University, Oxford, United Kingdom, ${ }^{2}$ UK Dementia Research Institute, Cardiff, United Kingdom

We sequenced $~ 17,000$ nuclei from both the Cortex and Substantia Nigra (SN) of five human brains and generated the first human single-nuclei transcriptomic atlas of the SN, a region playing important roles in reward and movement. By mapping genetic variants associated with different human traits to SN cell-type-specific gene expression patterns, we demonstrate for the first time that Parkinson's disease (PD) genetic risk, for which the symptoms are caused by loss of SN dopaminergic neurons (DaNs), is indeed associated with DaN-specific gene expression affecting pathways such as mitochondrial organisation and functioning, protein ubiquitination and vesicle transport. However, we also identify a distinct cell type association between PD risk and oligodendrocyte-specific gene expression. Unlike Alzheimer's disease (AD), we find no association between PD risk and microglia or astrocytes, suggesting that neuroinflammation plays a less causal role in PD than AD. Beyond PD, we find other neuropsychiatric disorders, particularly schizophrenia (SCZ), to be associated to SN DaNs and GABAergic neurons. Nevertheless, we find that each neuropsychiatric disorder is associated with a distinct set of genes within those neuronal types. On the contrary, we find an overlapping component of risk between neuropsychiatric disorders in the association to glial cells, particularly oligo-precursor cells (OPCs). This atlas provides the first objective associations between genetic risk of multiple disorders and the midbrain cell types these risks likely manifest through, thereby directing our aetiological understanding.

D. Agarwal: None. C. Sandor: None. V. Volpato: None. T. Caffrey: None. J. Monzon-Sandoval: None. R. Bowden: None. J. Alegre-Abarrategui: None. R. Wade-Martins: None. C. Webber: None. 


\section{C12.6}

\section{Clinical and molecular complexity of X-linked clustering epilepsy, a disorder of cellular mosaics}

\section{J. Gecz ${ }^{I^{*}, \text { K. Kolc }}{ }^{1}$, L. Sadleir ${ }^{2}$, C. Depienne ${ }^{3}$, C. Marini ${ }^{4}$, I. E. Scheffer ${ }^{5}$, R. S. Moller ${ }^{6}$, M. Trivisano ${ }^{7}$, N. Specchio ${ }^{7}$, R.

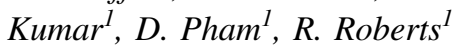

${ }^{1}$ The University of Adelaide, Adelaide, Australia, ${ }^{2}$ University of Otago, Wellington, New Zealand, ${ }^{3}$ University Hospital Essen, Essen, Germany, ${ }^{4}$ University of Florence, Florence, Italy, ${ }^{5}$ University of Melbourne, Melbourne, Australia, ${ }^{6}$ The Danish Epilepsy Centre, Dianalund, Denmark, ${ }^{7}$ Bambino Gesù Children's Hospital, Rome, Italy

PCDH19 is a well-established neurodevelopmental disability (NDD) gene implicated in an early onset seizure disorder, X-linked clustering epilepsy or XCE. XCE is one of the most frequent epilepsies. It is a disorder of cellular mosaics, i.e. heterozygous females (due to $\mathrm{X}$ inactivation mosaicism) or postzygotic somatic mosaic males. Hemizygous males without functional PCDH19 are not affected. In the present study we investigated the complexity of the XCE NDD phenotype as well as the cellular and molecular determinants of its variable $(\sim 80 \%)$ penetrance. Firstly, using standardized survey tools we have assessed executive function (Behavior Rating Inventory) and psychiatric comorbidities (Social Responsiveness Scale, Social Communication Questionnaire, Strengths and Difficulties Questionnaire, and Dimensional Obsessive Compulsive Scale) on 112 individuals with deleterious PCDH19 variant, males and females. Genetic, seizure, and developmental information were also collected. Developmental delay prior to seizure onset occurred in 18\%, executive dysfunction and ASD in $60 \%$ of individuals. $21 \%$ of individuals met criteria for obsessive-compulsive disorder, distinct from ASD. There were no phenotypic differences between heterozygous females and somatic mosaic males attesting to the cellular mosaicism as the disease driver. Secondly, we assessed 322 PCDH19 missense variants, 45 of which we tested experimentally using in vitro assays to engineer a high accuracy PCDH19 variant predictor toolbox. Thirdly, using patient-derived skin fibroblast cell lines we identified genetic (mRNA, protein) and epigenetic (methylation) biomarkers of the penetrance of XCE even among discordant monozygotic twins. Our research set foundations of a Phase III international clinical trial of ganaxolone in XCE.

J. Gecz: None. K. Kolc: None. L. Sadleir: None. C. Depienne: None. C. Marini: None. I. E. Scheffer: None. R.
S. Moller: None. M. Trivisano: None. N. Specchio: None. R. Kumar: None. D. Pham: None. R. Roberts: None.

\section{C13 3D Genome Architecture}

\section{C13.1}

Landscape of cohesin-mediated chromatin loops in the human genome

F. Grubert ${ }^{*}$, R. Srivas ${ }^{l}$, D. Spacek ${ }^{l}$, M. Kasowski ${ }^{l}, M$.

Ruiz-Velasco ${ }^{2}$, N. Sinnott-Armstrong ${ }^{1}$, P. Greenside ${ }^{l}, A$. Narasimha $^{l}$, Q. Liu ${ }^{l}$, B. Geller ${ }^{l}$, A. Sanghi ${ }^{l}$, M. Kulik ${ }^{3}$, S. $\mathrm{Sa}^{l}$, M. Rabinovitch ${ }^{l}$, A. Kundaje ${ }^{l}$, S. Dalton ${ }^{3}$, J. Zaugg $^{2}$, M. Snyder ${ }^{l}$

${ }^{1}$ Stanford University, Stanford, CA, United States, ${ }^{2} E M B L$, Heidelberg, Germany, ${ }^{3}$ University of Georgia, Athens, GA, United States

Physical interactions between distal regulatory elements in the genome play a key role in regulating gene expression, yet the extent to which these interactions vary between cell types and contribute to cell type-specific gene expression and how they are related to overall gene function remains unclear. To address these questions as part of ENCODE Phase III, we mapped cohesin-mediated chromatin loops, using a high-resolution ChIA-PET assay, and gene expression in 24 diverse human cell types, including core ENCODE cell lines. We find that $28 \%$ of all chromatin loops vary across cell types, and these changes are effective at grouping cell types based on their tissue of origin, indicating commonalities in three-dimensional (3D) genome architecture amongst related cell types. This also applied to different cell types that were derived from the same individual, demonstrating that the observed variation is mainly due to epigenetic changes. Cell type-specific loops show different patterns of chromatin states, with stem cell loops being depleted of active promoter states and transcription, and enriched in bivalent states. We further find that the connectivity of genes corresponds to different functional classes, with housekeeping genes being connected by few loops, and dosagesensitive genes being highly connected to enhancer elements. Finally, we demonstrate that genetic variants identified in GWAS are enriched in variable loops in disease-relevant cell types. Overall, our results provide important insights on how variation in 3D chromatin organization corresponds to cell type-specific functions and identity.

F. Grubert: None. R. Srivas: None. D. Spacek: None. M. Kasowski: None. M. Ruiz-Velasco: None. N. SinnottArmstrong: None. P. Greenside: None. A. Narasimha: None. Q. Liu: None. B. Geller: None. A. Sanghi: None. M. Kulik: None. S. Sa: None. M. Rabinovitch: None. A. Kundaje: None. S. Dalton: None. J. Zaugg: None. M. Snyder: None. 


\section{C13.2}

Analysis of chromatin looping at joint endometrialovarian cancer risk loci to identify candidate target genes

\section{Glubb*, I. Jeong, A. Spurdle, T. O’Mara}

QIMR Berghofer Medical Research Institute, Brisbane, Australia

We have previously identified 11 joint risk endometrialovarian cancer loci through analysis of genome-wide association study (GWAS) data from the two largest studies of these diseases. To find candidate target genes at joint risk loci, we performed HiChIP chromatin looping analysis of three endometrial and three ovarian cancer cell lines. To enrich for enhancer-promoter interactions, a mechanism through which GWAS-identified variation may target genes, we captured loops associated with H3K27Ac histone (characteristic of promoters and enhancers). We identified $60,208-182,001$ replicated loops per cell line, $28-44 \%$ of which contacted a promoter. Intersection of promoterassociated loops with statistically credible risk variants highlighted 59 genes, at eight joint risk loci, as candidate targets in both endometrial and ovarian cancer cell lines. These genes included those with well-established oncogenic roles (e.g. TERT, MYC and CCND1). Among the candidate target genes, there was a two-fold enrichment $(\mathrm{p}=0.01)$ for genes differentially expressed in endometrial or ovarian tumours. Network analysis prioritised a set of proteins that interact with those encoded by candidate target genes. This set was 16 -fold enriched $\left(\mathrm{p}=2 \times 10^{-15}\right)$ for endometrial or ovarian cancer drivers and included proteins encoded by mismatch repair genes $M L H 1, M S H 2$ and $M S H 6$, for which variants are penetrant for endometrial cancer and ovarian cancer. Lastly, candidate target genes and prioritised interacting proteins were over-represented in pathways related to endometrial and ovarian cancer development. In summary, we have identified biologically relevant candidate genes and pathways, providing insight into endometrial and ovarian cancer aetiology.

Funded by NHMRC project grant \#1158083 (DG and TOM)

D. Glubb: None. I. Jeong: None. A. Spurdle: None. T. O’Mara: None.

\section{C13.3}

When the silent genome gets loud: transcription of repeated genomic elements at the single-cell resolution in K27M-mutated high-grade gliomas

M. Coutelier ${ }^{1,2^{*}}$, V. Lisi ${ }^{2,3}$, S. Hébert ${ }^{1,2}$, D. Faury ${ }^{4,3}, B$.

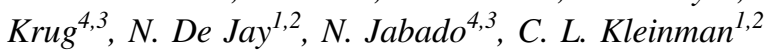

${ }^{l}$ Department of Human Genetics, McGilll University, Montreal, QC, Canada, ${ }^{2}$ Lady Davis Institute for Medical Research, Jewish General Hospital, Montreal, QC, Canada, ${ }^{3}$ The Research Institute of the McGill University Health Center, Montreal, QC, Canada, ${ }^{4}$ Department of Pediatrics, McGill University, Montreal, QC, Canada

Introduction: High-grade gliomas are a leading cause of cancer-related death in children and young adults, and no targeted treatments are currently available. A recurrent lysine-to-methionine substitution at position 27 of histone 3 variants has been shown to induce alterations of repressive and active chromatin landscapes, respectively a global loss of H3K27 trimethylation, and a pervasive gain of H3K27 acetylation. Repeated genomic elements are particularly targeted by this de-repression, leading to an exquisite sensitivity of $\mathrm{H} 3 \mathrm{~K} 27 \mathrm{M}$ cells to agents triggering viral mimicry.

Materials and methods: In K27M-mutated gliomas, we combine single-nuclei (sn)RNA-sequencing and snATACsequencing to investigate the intra-tumoral heterogeneity of genomic repeats expression.

Results: In several samples bearing K27M mutations in all three variants of histone 3, we consistently identified gradients of repeats expression. These gradients correlated with gene signatures specific of K27M gliomas, a result corroborated by snATAC-sequencing. Cells displaying high repeats expression also showed a higher enrichment for targets of the repressive methylation complex PRC2, as well as a transcriptomic profile resembling less differentiated oligodendrocyte progenitor cells.

Conclusions: We have implemented an approach to detect expression of genomic repeats at the single cell level and demonstrate gradients of expression in high-grade gliomas. Our results corroborate the existence of tumor compartments in K27M-tumors that consist of progenitorlike, aggressive malignant cells, displaying higher expression of the silent genome. They advocate for the potential of viral mimicry trigger as therapeutic option for these untreatable tumors.

M. Coutelier: None. V. Lisi: None. S. Hébert: None. D. Faury: None. B. Krug: None. N. De Jay: None. N. Jabado: None. C. L. Kleinman: None.

\section{C13.4}

$\mathrm{CDH1}$ regulatory noncoding elements: a hidden master for tissue-specific E-cadherin expression

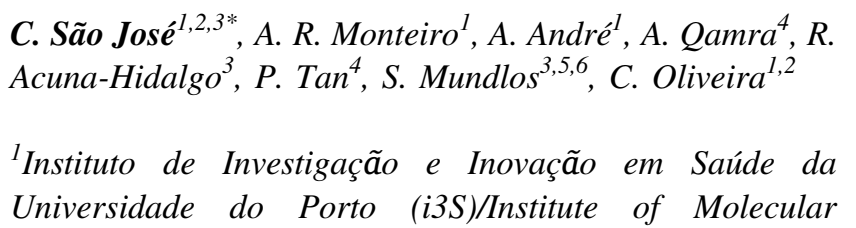


Pathology and Immunology of the University of Porto (Ipatimup), Porto, Portugal, ${ }^{2}$ Faculty of Medicine of the University of Porto, Porto, Portugal, ${ }^{3}$ Max Planck Institute for Molecular Genetics, Berlin, Germany, ${ }^{4}$ Cancer Science Institute of Singapore (NUS), Singapore, Singapore, ${ }^{5}$ Institute for Medical and Human Genetics, Charité Universitätsmedizin, Berlin, Germany, ${ }^{6}$ Berlin-Brandenburg Center for Regenerative Therapies (BCRT), Charité Universitätsmedizin, Berlin, Germany

Introduction: Germline $\mathrm{CDH} 1 / \mathrm{E}-\mathrm{cadherin}$ loss of function (LoF) causes inherited gastric and breast cancer; and somatic LoF occurs during progression of most epithelial cancers. Classical genetic/epigenetic/post-transcriptional inactivation mechanisms fail to explain $\mathrm{CDH} 1 \mathrm{LoF}$ in most inherited/somatic contexts. We hypothesized that $\mathrm{CDHI}$ LoF occurs due to defects in the $\mathrm{CDH} 1$ regulatory network and explored potential expression modifier sequences controlling $\mathrm{CDH} 1$ expression.

Materials and Methods: Six gastric-cancer cell lines were submitted to Capture $\mathrm{Hi}-\mathrm{C}(\mathrm{cHi}-\mathrm{C})$ and $4 \mathrm{C}$-seq with a $\mathrm{CDH} 1$ promoter viewpoint. Interactors' expression patterns in normal tissues were explored in GTex. Interactors' regulatory function was assessed in mouse embryo LacZreporter assays, followed by tissue-specific $\beta$-galactosidase expression analysis in transgenic embryonic stomach, esophagus, duodenum, liver (endoderm), heart (ectoderm) and skin (mesoderm).

Results: We found simultaneous interactions between CDH1 promotor and (1) a new IncRNA at chromosome\#2 short-arm, and (2) a CDH1-intronic cis-regulatory element (CDH1-iCRE). These were specific for $\mathrm{CDH1}$ non-expressing cells, and undetected in $\mathrm{CDH} 1$-expressing cells, highlighting a potential negative regulatory network. GTEx data revealed that IncRNA and $\mathrm{CDH} 1$ have mutually exclusive expression patterns, with $\mathrm{CDHl}$ being expressed across epithelial tissues, and the lncRNA restricted to brain and testis. $C D H 1$ expression was 100-fold higher than lncRNA expression. The interacting $\mathrm{CDH}$ l-iCRE showed tissue-specificity in endodermal-derived tissues where Ecadherin exerts a primordial function (stomach, esophagus and duodenum).

Conclusion: We found a potential negative regulatory network in gastric cancer cell lines through $\mathrm{CDH} 1$ promoter cis and trans interactions, and evidence for tissue-specific regulation. These findings suggest a novel mechanism triggering $\mathrm{CDH} / \mathrm{E}$-cadherin $\mathrm{LoF}$.

C. São José: None. A. R. Monteiro: None. A. André: None. A. Qamra: None. R. Acuna-Hidalgo: None. P. Tan: None. S. Mundlos: None. C. Oliveira: None.

\section{C13.5}

Local gene co-expression: molecular characterisation, tissue specificity and its genetic control

\author{
D. Ribeiro ${ }^{1,2^{*}}$, A. Ramisch $^{3,2}$, E. Dermitzakis ${ }^{3,2}, O$. \\ Delaneau ${ }^{l, 2}$
}

${ }^{1}$ University of Lausanne, Lausanne, Switzerland, ${ }^{2}$ Swiss Institute of Bioinformatics (SIB), Lausanne, Switzerland, ${ }^{3}$ University of Geneva, Geneva, Switzerland

Many Eukaryotic genes tend to be expressed together. Recent studies highlight the existence of structural and regulatory domains orchestrating the organised expression of nearby genes (Delaneau et al. 2019 Science 364(6439)). Yet, the molecular mechanisms, genetic control and tissue specificity of local gene co-expression are far from being understood. Here, we developed a novel method to identify groups of nearby genes (within $1 \mathrm{Mb}$ ) that are co-expressed, using genome-wide RNA-seq datasets from large populations (e.g. $>200$ individuals). First, we identified 9548 highconfidence co-expressed gene pairs (FDR 1\%) from 358 human lymphoblastoid cell line (LCL) samples from the Geuvadis dataset. These constitute $52.5 \%$ of the proteincoding and lincRNA genes expressed in LCLs, demonstrating that local gene co-expression is highly prevalent. Next, by integrating various molecular assays (e.g. ChIPseq, Hi-C), we determined the relative contribution of several molecular features towards local gene co-expression. For instance, we found that CTCF binding plays a role in determining which gene pairs can be co-expressed, whereas chromatin contacts between genes shown to be key only when these are distant $(>200 \mathrm{~kb})$ from each other. Notably, developing novel QTL mapping approaches, we found that the co-expression of more than $50 \%$ of the gene pairs is controlled by nearby genetic variants (co-QTLs), many of whom falling within shared regulatory elements, such as enhancers. Finally, we applied our novel approaches across 53 human GTEx tissues to study in-depth the tissuespecificity and genetic control of local gene co-expression, ultimately improving the functional interpretation of QTL and GWAS findings.

D. Ribeiro: None. A. Ramisch: None. E. Dermitzakis: None. O. Delaneau: None.

\section{C13.6}

Structural variants in HDAC9 that disrupt TWIST1 transcriptional regulation but not HDAC9 protein function are associated with craniofacial and limb malformations

R. Y. Birnbaum ${ }^{1,2 *}, N$. Hirsch $^{3}$ 
${ }^{1}$ Department of Life Sciences, Faculty of Natural Sciences, The Ben-Gurion University of the Negev, Israel., BeerSheva, Israel, ${ }^{2}$ Center of Evolutionary Genomics and Medicine, The Ben-Gurion University of the Negev, Beer Sheva, Israel, ${ }^{3}$ Department of Life Sciences, Faculty of Natural Sciences, The Ben-Gurion University of the Negev, Beer-Sheva, Israel

Structural variants (indels, translocations and inversions) are associated with human variations. SVs affecting protein coding sequences andlor regulatory elements are associated with human disorders. However, SVs disrupting protein coding sequences that also function as transcriptional regulation are yet to be demonstrated. Here, we show that patients with SVs involving HDAC9 protein coding sequence are associated with craniosynostosis due to disruption of TWIST1 regulatory elements that reside in HDAC9 sequence and not due to disruption of HDAC9 protein. By generating CRISPRICas9 mouse models, we deleted Hdac9 protein coding sequence that also function as Twist1 transcription enhancers $\left(\mathbf{e} \mathbf{T w 5}-\mathbf{7}^{\mathbf{\Delta} / \mathbf{\Delta}}\right.$ ) that altered Twistl expression and led to significant craniofacial phenotype and polydactyly, resemble Saethre-Chotzen syndrome. Furthermore, deletion of Hdac 9 sequence encompasses CTCF site $\left(\mathbf{C T C F}^{\mathbf{\Delta} / \mathbf{\Delta}}\right)$ disrupted Twist enhancer-promoter interactions which led to craniofacial phenotype and polydactyly. Using RNA-seq and 4C-seq, we demonstrate that in both Hdac9 deletion models Twist1 expression, but not Hdac9, was reduced and the effect on the downstream targets of Twist1 elucidated unexpected pathways. Finally, we generated a large inversion of the entire Hdac9 sequence (Hdac9 ${ }^{\mathbf{I N V} /+}$ ) that does not disrupt Hdac9 coding sequence but rather reposition Twist1 regulatory elements. We showed reduction of Twist1 expression, but not of Hdac9, that led to craniofacial phenotype and polydactyly. Thus, we elucidated the TWISTI transcriptional machinery that reside in HDAC9 sequence, suggesting that SVs encompass protein coding sequences could lead to a phenotype that is not attributed to the protein function but rather a disruption of the transcription regulation of nearby gene, such as TWIST1.

R. Y. Birnbaum: None. N. Hirsch: None.

\section{C14 Internal Organs}

\section{C14.1}

\section{Implication of $\mathrm{FOXD2}$ in autosomal recessive syndromic CAKUT}

K. M. Riedhammer ${ }^{1,2 *}, M$. Nguyen $^{3}, B$. Alhaddad $^{l}, S . J$. Arnold $^{4}$, G. J. Kim ${ }^{4}$, U. Heemann ${ }^{2}$, M. Schmidts ${ }^{5}, J$. Hoefele $^{l}$
${ }^{1}$ Institute of Human Genetics, Klinikum rechts der Isar, Technical University of Munich, Munich, Germany, ${ }^{2}$ Department of Nephrology, Klinikum rechts der Isar, Technical University of Munich, Munich, Germany, ${ }^{3}$ Department of Human Genetics, Radboud University Medical Center, Nijmegen, Netherlands, ${ }^{4}$ Institute of Experimental and Clinical Pharmacology and Toxicology, Faculty of Medicine, University of Freiburg, Freiburg, Germany, ${ }^{5}$ Center for Pediatrics and Adolescent Medicine, Freiburg University Hospital, University of Freiburg, Freiburg, Germany

Introduction: $50 \%$ of chronic kidney disease up to age 30 can be attributed to congenital anomalies of the kidney and urinary tract (CAKUT). CAKUT is genetically complex: Although more than 40 genes have been linked to monogenic CAKUT and familial clustering is frequent, monogenic causes can only be identified in about $12 \%$ of cases. Exome sequencing (ES) has become a valuable tool for the analysis of genetically heterogeneous diseases both in diagnostics and research. We report a multiplex consanguineous Arab family with several individuals segregating syndromic kidney hypoplasia in which ES identified a homozygous frameshift variant in FOXD2 and show functional characterization of Foxd2 knockout (KO).

Material \& Methods: Analysis of the index patient by ES, Sanger sequencing of relatives. CRISPR/Cas9-mediated KO of Foxd2 (Foxd2 $\left.{ }^{-I-}\right)$ in murine metanephric mesenchyme cells (mk4). RNA sequencing of KO cells.

Results: ES identified a homozygous frameshift variant in FOXD2 NM_004474.3:c.789dup, p.(Gly264Argfs*228) in the index patient (bilateral hypoplastic kidneys, facial dysmorphism, developmental delay). Sanger sequencing confirmed segregation of the variant in the family. Pathway analysis of RNA data from Foxd $2^{-1-}$ cells vs. WT revealed significant enrichment of differential expression of renal developmental genes. Pax2 and Wnt4 were notable significantly downregulated genes.

Discussion: FOXD2 is a transcription factor with strong expression in the developing kidney. Foxd $2^{-/-}$mice can show a CAKUT phenotype. Our data implicate FOXD2 in autosomal recessive syndromic CAKUT. RNA sequencing results suggest that $\mathrm{KO}$ of Foxd2 leads to impaired transition from metanephric mesenchyme to epithelium in the developing kidney via the Pax2-Wnt4 axis.

K. M. Riedhammer: None. M. Nguyen: None. B. Alhaddad: None. S. J. Arnold: None. G. J. Kim: None. U. Heemann: None. M. Schmidts: None. J. Hoefele: None. 


\section{C14.2}

Heterozygous DACT1 mutations in patients with renal anomalies and features of Townes-Brocks syndrome

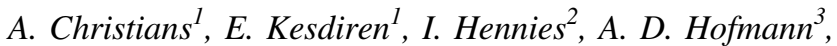

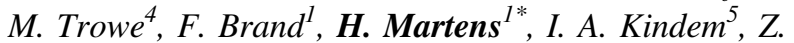

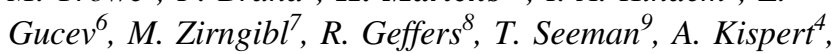
H. Billing ${ }^{7}$, A. Bjerre ${ }^{5}$, V. Tasic ${ }^{6}$, D. Haffner ${ }^{2}, J$.

Dingemann $^{3}$, R. G. Weber ${ }^{l}$

${ }^{I}$ Department of Human Genetics, Hannover Medical School, Hannover, Germany, ${ }^{2}$ Department of Pediatric Kidney, Liver and Metabolic Diseases, Hannover Medical School, Hannover, Germany, ${ }^{3}$ Department of Pediatric Surgery, Hannover Medical School, Hannover, Germany, ${ }^{4}$ Institute of Molecular Biology, Hannover Medical School, Hannover, Germany, ${ }^{5}$ Division of Pediatric and Adolescent Medicine, Oslo University Hospital, Oslo, Norway, ${ }^{6} \mathrm{Med}$ ical Faculty Skopje, University Children's Hospital, Skopje, Macedonia, The Former Yugoslav Republic of, ${ }^{7}$ Department of Pediatric Nephrology, University Children's Hospital Tübingen, Tübingen, Germany, ${ }^{8}$ Genome Analytics Research Group, Helmholtz Centre for Infection Research, Braunschweig, Germany, ${ }^{9}$ Department of Pediatrics, Second Faculty of Medicine, Charles University Prague, Prague, Czech Republic

Less than $20 \%$ of patients with congenital anomalies of the kidney and urinary tract (CAKUT) carry variants in the approximately 50 known CAKUT genes, indicating that this disorder is genetically highly heterogeneous. To identify novel genes associated with CAKUT, we applied whole-exome sequencing to a patient with renal anomalies, i.e. left-sided agenesis and right-sided duplex, who also had extrarenal abnormalities, e.g. anorectal and sacral malformation, thus detecting a rare heterozygous missense variant in the dishevelled binding antagonist of beta catenin 1 (DACT1) gene. As combined renal, anorectal, and skeletal defects, features overlapping Townes-Brocks syndrome, were previously described in a family carrying a $D A C T 1$ nonsense variant and in Dactl-deficient mice, the identified DACT1 variant was presumed causative in our patient. Therefore, we aimed to determine the frequency of $D A C T 1$ variants in patients with renal anomalies. Taken together, we identified seven maternally inherited, heterozygous, likely pathogenic DACT1 missense variants in 8 of 209 families (3.8\%) with renal agenesis/dysplasia/hypoplasia and other features of Townes-Brocks syndrome. Immunoprecipitation revealed reduced dishevelled segment polarity protein 2 (DVL2) binding capacity of mutant DACT1. By RNA in situ hybridization on murine embryo sections, Dactl expression was detected in different organs including anal canal and kidney, whereby renal expression was confined to the mesenchyme of ureter, kidney capsule, cortical and medullary stroma. CRISPR/Cas9-derived Dactl-deficient murine inner medullary collecting duct cells showed impaired tubule formation. Altogether, we demonstrate a statistically significant overrepresentation of rare heterozygous hypomorphic $D A C T 1$ variants in patients with renal anomalies versus controls. (DFG grant no. KO5614/21)

A. Christians: None. E. Kesdiren: None. I. Hennies: None. A. D. Hofmann: None. M. Trowe: None. F. Brand: None. H. Martens: None. I. A. Kindem: None. Z. Gucev: None. M. Zirngibl: None. R. Geffers: None. T. Seeman: None. A. Kispert: None. H. Billing: None. A. Bjerre: None. V. Tasic: None. D. Haffner: None. J. Dingemann: None. R. G. Weber: None.

\section{C14.3}

Loss of UNC45A causes microvillus inclusion diseaselike by impairing myosin-dependent epithelial morphogenesis

R. Duclaux-Loras ${ }^{I}$, F. Charbit-Henrion ${ }^{l}$, C. Lebreton ${ }^{l}$, O. Nicolle $^{2}$, M. Rabant ${ }^{3}$, C. Guerrera ${ }^{4}$, A. Fabre ${ }^{5}$, L. Faivre ${ }^{6}$, G. Michaux ${ }^{2}$, H. Uligh ${ }^{7}$, F. Ruemmele ${ }^{3}$, N. CerfBensussan $^{1}$, M. Parlato ${ }^{1 *}$

${ }^{1}$ Institut Imagine, Paris, France, ${ }^{2}$ CNRS, UMR6290, Institut de Génétique et Développement de Rennes, Rennes, France, ${ }^{3}$ Assistance Publique-Hôpitaux de Paris, Hôpital NeckerEnfants Malades, Department of Pediatric Gastroenterology, Paris, France, ${ }^{4}$ Proteomics platform 3P5-Necker, Université Paris Descartes-Structure Fédérative de Recherche Necker, INSERM US24/CNRS UMS3633, Paris, France, ${ }^{5}$ Service de pédiatrie multidisciplinaire, Hôpital de la Timone Enfants, APHM, Marseille, France, ${ }^{6}$ Centre de Génétique, Hôpital d'Enfants, CHU Dijon, Dijon, France, ${ }^{7}$ Department of Pediatrics, University of Oxford; Translational Gastroenterology Unit, University of Oxford, Oxford, United Kingdom

Introduction: Congenital diarrhea disorders (CDDs) are rare inherited diseases characterized by chronic enteropathy starting early in life. Recently, mutations in UNC45A, coding for a myosins- co-chaperone, have been associated with a complex syndrome combining cholestasis, diarrhea, deafness and bone fragility. We aimed to investigate the pathomechanism underlying the intestinal defect in UNC45A deficient patients.

Materials and Methods: We investigated 4 unrelated patients with history of intestinal failure due to congenital diarrhea. We used next generation sequencing to uncover the underlying gene defect and immunohistochemistry/ electron microscopy to analyse intestinal tissue. UNC45A 
$\mathrm{CaCo} 2$ cells were generated by CRISPR/Cas9 editing and cultured in matrigel to form cysts. UNC45A interactome was investigated by mass spectrometry. Organoids were generated from controls and from patients using blood-derived pluripotent stem cells.

Results: Exome analysis identified biallelic mutations in UNC45A in all patients. Abnormal PAS and CD10 accumulation throughout the subapical cytoplasm was found on patient duodenal biopsies. Microvesicles and microvilli inclusions were associated with microvillous atrophy. UNC45 $\mathrm{A}^{-1} \mathrm{CaCo} 2$ cells developed into multilumens-cysts, indicating a defect in epithelial morphogenesis. Mass spectrometry confirmed MYO5B as UNC45A-HSP90 client and expanded the number of non-muscle myosins-II, dependent on UNC45A for proper folding and maturation. Patient's organoids displayed features of microvillus inclusion disease.

Conclusions: We identified 6 novel mutations in UNC45A causative of a complex syndrome including congenital diarrhea. EM on patient derived organoids recapitulates the defects of morphology and polarization observed in patient's biopsies. Overall, our results underscore the important role of UNC45A in shaping epithelial morphogenesis in the gut.

R. Duclaux-Loras: None. F. Charbit-Henrion: None. C. Lebreton: None. O. Nicolle: None. M. Rabant: None. C. Guerrera: None. A. Fabre: None. L. Faivre: None. G. Michaux: None. H. Uligh: None. F. Ruemmele: None. N. Cerf-Bensussan: None. M. Parlato: None.

\section{C14.4}

A comprehensive exome study of the genetic architecture of asthma reveals a putative novel patient subgroup defined by filaggrin truncating variants

S. R. Cameron-Christie ${ }^{{ }^{*}}, H$. Olsson ${ }^{2}, A$. Mackay $^{2}, Q$.

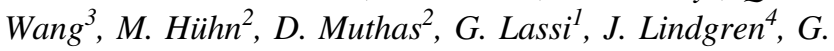
Povysil $^{5}$, D. B. Goldstein ${ }^{5}$, G. Belfield ${ }^{4}$, I. Dillmann ${ }^{4}, Y$.

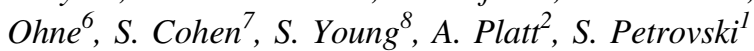

${ }^{1}$ Centre for Genomics Research, Precision Medicine and Genomics, IMED Biotech Unit, AstraZeneca, Cambridge, United Kingdom, ${ }^{2}$ Translational Science and Experimental Medicine, Research and Early Development, Respiratory, Inflammation and Autoimmune (RIA), R\&D BioPharmaceuticals, AstraZeneca, Cambridge, UK, Gothenburg, Sweden, ${ }^{3}$ Centre for Genomics Research, Precision Medicine and Genomics, IMED Biotech Unit, AstraZeneca, Waltham, MA, United States, ${ }^{4}$ Translational Genomics, Discovery Sciences, R\&D BioPharmaceuticals, AstraZeneca, Gothenburg, Sweden, Gothenburg, Sweden, Institute for Genomic Medicine, Columbia University Irving Medical Center, New York, New York, New York, NY, United States,
${ }^{6}$ Bioscience Asthma, Research and Early Development, Respiratory, Inflammation and Autoimmune (RIA), BioPharmaceuticals R\&D, AstraZeneca, Gothenburg, Sweden, ${ }^{7}$ Bioscience Asthma, Research and Early Development, Respiratory, Inflammation and Autoimmune (RIA), BioPharmaceuticals $R \& D$, AstraZeneca, Cambridge, United Kingdom, ${ }^{8}$ Precision Medicine, Oncology R\&D, AstraZeneca, Cambridge, UK, Cambridge, United Kingdom

Asthma is a highly heterogenous disease with considerable unmet need. Understanding the genetic architecture may enable a precision medicine approach. We studied exome data from 24,584 asthma cases from the UKBiobank (UKB) and SAGA, an AstraZeneca study. Controls were 120,557 UKB participants with FEV/FVC ratio $>70 \%$, without respiratory illness. In addition to all-comer asthma $(n=24,584)$, we studied familial $(\mathrm{n}=1,065)$ and early-onset $($ age $<18 \mathrm{yrs}, \mathrm{n}=$ $5,962)$ asthma subsets. Gene- and variant-level analyses identified statistically significant signals from moderate-to-common protein-truncating variants (PTVs), including in $F L G$, which has previously been associated with asthma. Asthma risk was significantly increased not only by common $F L G$ PTVs, but also the collection of rare-to-private $F L G$ PTVs. This signal was driven by early onset asthma, with no measurable impact on diagnoses $>18$ years old $(p=0.91, O R=1.00)$. Risk was strongest among atopic asthma (diagnoses $<18$ y.o., $\mathrm{p}=$ $1.13 \times 10^{-37}, \mathrm{OR}=2.32$ ), but remained significant after correcting for allergies and related phenotypes $\left(\mathrm{p}=1.26 \times 10^{-13}\right.$, $\mathrm{OR}=1.4)$. Finally, we found $F L G$-associated asthma does not correlate with eosinophil levels. In addition, a single splice variant in IL33, rs146597587-C, previously proposed as a protective, loss-of-function PTV (Smith $D$ (2017) PLoS Genet), was replicated as protective $\left(\mathrm{p}=8.0 \times 10^{-10}, \mathrm{OR}=\right.$ 0.58 ). We further observed significantly lower carrier rates of this splice-variant among early-onset asthmatics (0.36\%) compared to later onset $(0.61 \%)$ (Mann-Whitney $\mathrm{p}=0.001)$. While we did not find evidence of novel asthma genes among current samples, we did identify a putative novel patient subgroup from the recognised $F L G$ asthma loci, namely $F L G$ PTV carriers, whose prevalence in UKB is $12 \%$.

S. R. Cameron-Christie: A. Employment (full or parttime); Significant; AstraZeneca. H. Olsson: A. Employment (full or part-time); Significant; AstraZeneca. A. Mackay: A. Employment (full or part-time); Significant; AstraZeneca. Q. Wang: A. Employment (full or part-time); Significant; AstraZeneca. M. Hühn: A. Employment (full or part-time); Significant; AstraZeneca. D. Muthas: A. Employment (full or part-time); Significant; AstraZeneca. G. Lassi: A. Employment (full or part-time); Significant; AstraZeneca. J. Lindgren: A. Employment (full or part-time); Significant; AstraZeneca. G. Povysil: None. D. B. Goldstein: F. Consultant/Advisory Board; Modest; AstraZeneca. G. Belfield: A. Employment (full or part-time); Significant; 
AstraZeneca. I. Dillmann: A. Employment (full or part-time); Significant; AstraZeneca. Y. Ohne: A. Employment (full or part-time); Significant; AstraZeneca. S. Cohen: A. Employment (full or part-time); Significant; AstraZeneca. S. Young: A. Employment (full or part-time); Significant; AstraZeneca. A. Platt: A. Employment (full or part-time); Significant; AstraZeneca. S. Petrovski: A. Employment (full or parttime); Significant; AstraZeneca.

\section{C14.5}

Random plasma glucose GWAS in 479,678 individuals: genetic relationships with impaired lung function and intestinal health

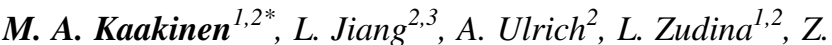

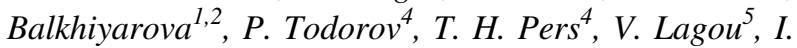 Prokopenko $^{1,2}$, MAGIC Investigators}

${ }^{1}$ University of Surrey, Guildford, United Kingdom, ${ }^{2}$ Imperial College London, London, United Kingdom, ${ }^{3}$ The University of Queensland, Brisbane, Australia, ${ }^{4}$ University of Copenhagen, Copenhagen, Denmark, ${ }^{5}$ VIB Center for Brain \& Disease Research, Leuven, Belgium

Fasting glucose and 2-hour post-prandial glucose are used as the gold standard tests for diagnosing type 2 diabetes (T2D). High non-fasting i.e. random plasma glucose (RG) levels may also indicate abnormal glucose homeostasis. We sought to characterise the genetic architecture of RG and its genetic relationships with other traits by performing a genome-wide association study of $8.1 \mathrm{M}$ SNPs with $\mathrm{MAF}>1 \%$ for $\mathrm{RG}$ in non-diabetic individuals from 17 European studies, including UK Biobank, adjusted for i) age, sex, population structure $(\mathrm{N}=479,678)$ and ii) additionally for time since last meal $(\mathrm{N}=438,678)$. We evaluated the results from each model and their combination. The LD score regression was used to evaluate the genetic correlation between RG and other traits. We identified 156 signals at 117 loci associated with RG at genome-wide significance $\left(P<5 \times 10^{-8}\right)$, including 38 novel signals for glycaemic traits and T2D. These include five common coding variants within GCKR, TET2, RREB1, NMT1 and $W I P I 1$, and three low-frequency nonsynonymous variants within EDEM3, NEURODI and GLPIR genes. The LD score regression revealed significant genetic correlations between RG and glycaemic/obesity traits and T2D, as well as a novel inverse genetic correlation with forced vital capacity $\left(r_{\mathrm{g}}=-0.088, \mathrm{SE}=0.022, P=6.04 \times 10^{-5}\right)$. The latter finding brings molecular clues to the observational studies showing an impairment in lung function in individuals with T2D. DEPICT analysis of large-scale human expression data showed significant enrichment of genes at RG-associations in ileum and pancreas, indicating a role of the digestive tract in $\mathrm{RG}$ level regulation.

Funding: H2020-SC1-HBC-28-2019-LONGITOOLS, WCRF-2017/1641.

M. A. Kaakinen: None. L. Jiang: None. A. Ulrich: None. L. Zudina: None. Z. Balkhiyarova: None. P. Todorov: None. T. H. Pers: None. V. Lagou: None. I. Prokopenko: None.

\section{C14.6}

A complementary study approach unravels novel players in the pathoetiology of Hirschsprung's disease

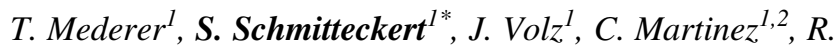
Röth $^{1,3}$, T. Thumberger ${ }^{4}$, V. Eckstein ${ }^{5}$, J. Scheuerer ${ }^{6}, C$. Thöni ${ }^{6}, F$. Lasitschka ${ }^{6}$, L. Carstensen ${ }^{7}$, P. Günther ${ }^{7}, S$. Holland-Cunz ${ }^{8}$, R. Hofstra ${ }^{9}$, E. Brosens ${ }^{9}$, D. Schriemer ${ }^{10}$, I. Ceccherini $^{11}$, M. Rusmini ${ }^{11}$, J. Tilghman ${ }^{12}$, B. LuzónToro $^{13,14}$, A. Torroglosa ${ }^{13,14}$, S. Borrego ${ }^{13,14}$, C. Szeman Tang $^{15}$, M. Garcia-Barcelo ${ }^{15}$, P. Tam ${ }^{15}$, N. Paramasivam ${ }^{16}$, M. Bewerunge-Hudler ${ }^{17}$, C. de la Torre ${ }^{18}$, N. Gretz $^{18}$, G. Rappold $^{1,19}$, P. Romero ${ }^{7}$, B. Niesler ${ }^{1,3,19}$

${ }^{1}$ Department of Human Molecular Genetics, Heidelberg University Hospital, Heidelberg, Germany, ${ }^{2}$ Lleida Institute for Biomedical Research Dr. Pifarré Foundation, Lleida, Spain, ${ }^{3}$ Counter Core Facility, Department of Human Molecular Genetics, Heidelberg University Hospital, Heidelberg, Germany, ${ }^{4}$ Center for Organismal Studies, Heidelberg University, Heidelberg, Germany, ${ }^{5}$ FACS Core Facility, Campus Heidelberg, Heidelberg, Germany, ${ }^{6}$ Institute of Pathology, Heidelberg University Hospital, Heidelberg, Germany, ${ }^{7}$ Pediatric Surgery Division, Heidelberg University Hospital, Heidelberg, Germany, ${ }^{8}$ Pediatric Surgery, University Children's Hospital, Basel, Switzerland, ${ }^{9}$ Department of Clinical Genetics, Erasmus University Medical Center, Rotterdam, Netherlands, ${ }^{10}$ Department of Neuroscience, University Medical Center, Groningen, Netherlands, ${ }^{11}$ UOSD Genetica e Genomica delle Malattie Rare, IRCCS, Instituto Giannina Gaslini, Genova, Italy, ${ }^{12}$ Center for Human Genetics and Genomics, New York University School of Medicine, New York, NY, United States, ${ }^{13}$ Department of Maternofetal Medicine, Genetics and Reproduction, Institute of Biomedicine of Seville (IBIS), University Hospital Virgen del Rocío/CSIC/University of Seville, Seville, Spain, ${ }^{14}$ Centre for Biomedical Network Research on Rare Diseases (CIBERER), Seville, Spain, ${ }^{15}$ Department of Surgery, Li Ka Shing Faculty of Medicine, The University of Hong Kong, Hong Kong, China, ${ }^{16}$ Division of Theoretical Bioinformatics, German Cancer Research Center, Heidelberg, Germany, ${ }^{17}$ Genomics and 
Proteomic Core Facility, German Cancer Research Center, Heidelberg, Germany, ${ }^{18}$ Center of Medical Research, Medical Faculty Mannheim, Mannheim, Germany, ${ }^{19}$ Interdisciplinary Center for Neurosciences, University of Heidelberg, Heidelberg, Germany

Introduction: Hirschsprung's disease (HSCR, OMIM 142623) involves congenital intestinal obstruction caused by dysfunction of neural crest cells and their progeny during enteric nervous system (ENS) development. HSCR is a multifactorial disorder and risk genes have only been identified in a minority of cases. Due to its complex genetic architecture, defining novel disease-relevant gene variants remains challenging.

Material and Methods: To identify candidate genes, whole exome sequencing (WES) of two HSCR patients and their non-affected parents was performed. To examine their disease-causing relevance, a complementary study approach has been established. This included transcriptome analysis of murine embryonic ENS-relevant tissues, in silico network analyses, expression analyses as well as functional assays assessing migration, differentiation, proliferation and cell survival capability of genome-edited cell clones.

Results: WES data were nailed down to four novel HSCR candidate genes: ATP7A, SREBF1, ABCDI and $P I A S 2$. Further rare variants in these genes were identified in additional HSCR patients, indicating disease relevance. In addition, transcriptomics revealed that these genes are expressed in embryonic and fetal gastrointestinal tissues. Functional analyses using candidate gene-specific, neuronal-like CRISPR/Cas9-edited knockout cell clones demonstrated impaired differentiation, proliferation and/or cell survival capacity.

Conclusions: Our approach identified and validated candidate HSCR genes and provided further insight into underlying pathomechanisms of HSCR.

Major Funding: This study was supported by the Heidelberg Stiftung Chirurgie, the Heinz and Heide Dürr Stiftung and the Dres. Majic/Majic Schlez Stiftung.

T. Mederer: None. S. Schmitteckert: None. J. Volz: None. C. Martinez: None. R. Röth: None. T. Thumberger: None. V. Eckstein: None. J. Scheuerer: None. C. Thöni: None. F. Lasitschka: None. L. Carstensen: None. P. Günther: None. S. Holland-Cunz: None. R. Hofstra: None. E. Brosens: None. D. Schriemer: None. I. Ceccherini: None. M. Rusmini: None. J. Tilghman: None. B. Luzón-Toro: None. A. Torroglosa: None. S. Borrego: None. C. Szeman Tang: None. M. Garcia-Barcelo: None. P. Tam: None. N. Paramasivam: None. M. Bewerunge-Hudler: None. C. de la Torre: None. N. Gretz: None. G. Rappold: None. P. Romero: None. B. Niesler: None.

\section{C15 Best Posters 1}

\section{C15.01}

START: The STudy of Assisted Reproductive Technologies

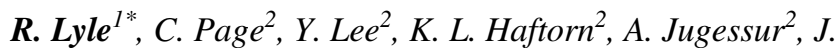

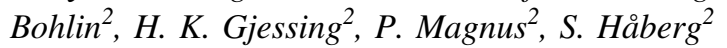

${ }^{1}$ Oslo University Hospital, Oslo, Norway, ${ }^{2}$ Norwegian Institute of Public Health, Oslo, Norway

In the last few decades there have been marked changes in patterns of fertility and family structure in rich countries. These include increasing age at childbirth, a lower number of children born to each woman or man, greater use of assisted reproductive technology (ART), higher frequency of family disruptions and increasingly complex family structures. The Centre for Fertility and Health $(\mathrm{CeFH}$; www.cefh.no) consists of a multidisciplinary research team of epidemiologists, geneticists, demographers, sociologists and economists with research themes including fertility problems and intergenerational transmission of health. The START project aims to understand the causes and health consequences of subfertility and how genetic influences and epigenetic differences are associated with subfertility and Assisted Reproductive Technologies (ART). ART may introduce epigenetic changes in the embryo since the procedures are during a period of extensive epigenetic repatterning. It is unknown which genes may be affected and the potential health consequences for the children. START uses data from the Norwegian Mother, Father and Child Cohort Study (MoBa; https://www.fhi.no/en/studies/moba/), which follows more than 110000 children and their parents. We have collected genetic and epigenetic data from 1000 trios with children born after ART, and 1000 trios from naturally conceived children analyzed with the Illumina EPIC array and the Illumina HumanCoreExome chip. Investigations are focussed on the effect of ART treatment on imprinted genes and fertility genes, and how epigenetic age acceleration interacts with fertility and ART treatment. Our results show DNA methylation changes due to both parental subfertility and ART procedures.

R. Lyle: None. C. Page: None. Y. Lee: None. K. L. Haftorn: None. A. Jugessur: None. J. Bohlin: None. H. K. Gjessing: None. P. Magnus: None. S. Håberg: None.

\section{C15.02}

Evaluating diagnostic challenges with ABCA4-related retinal disease - experience with a 7500 IRD patient cohort sent for genetic diagnostics 
J. Känsäkoski*, K. Kämpjärvi, S. Tuupanen, K. Wells, L. Sarantaus, P. von Nandelstadh, K. Merkkiniemi, $H$. Västinsalo, E. Mårtensson, R. Perez Carro, E. Sankila, J. W. Koskenvuo, S. Myllykangas, T. Alastalo

\section{Blueprint Genetics, Espoo, Finland}

Introduction: $\mathrm{ABCA} 4$ variants cause autosomal recessive Stargardt disease (STGD1), the most prevalent hereditary macular disease. Major challenges in STGD1 molecular diagnostics include (i) non-coding variants typically not targeted by available genetic tests (ii) copy number variants (CNV) missed by standard testing, and (iii) interpretation challenges with common hypomorphic variants. We evaluated the significance of challenging ABCA4 variants and their contribution to STGD1 diagnostic yield in a cohort of inherited retinal disease (IRD) patients.

Materials and Methods: We included 7500 IRD patients tested at a CLIA laboratory between 2017 and 2019 . Sequencing analysis, including established non-coding ABCA4 variants and high resolution $\mathrm{CNV}$ detection, was done by using in-house developed and validated NGS platform.

Results: Two disease-causing ABCA4 variants were identified in 663 patients, of which $20(20 / 663 ; 3 \%)$ had an established non-coding variant and $11(11 / 663 ; 1.5 \%)$ a CNV. In 192 patients with clinically diagnosed ABCA4related disease, only one disease-causing variant was identified. Among those, the hypomorphic allele ABCA4 c.5603A > T, p.(Asn1868Ile) was identified in 109 patients (109/192: 57\%), with a significantly increased allele frequency $(126 / 384=0.328)$ compared to controls in gnomAD (AF 11928/282712=0.042) (OR: 11.087, 95\% CI: $8.953-13.73, \mathrm{p}<0.001)$. The median age at genetic testing among the 109 patients was 56 years, which was significantly higher compared to 37 years among the 663 patients with two disease-causing variants.

Conclusions: Non-coding variants and CNVs contribute significantly to diagnostic yield in ABCA4-related disease. Our large dataset supports the important role of the hypomorphic allele ABCA4 c.5603A $>$ T, p.(Asn1868Ile) in diagnostics of late-onset STGD1.

J. Känsäkoski: A. Employment (full or part-time); Significant; Blueprint Genetics. K. Kämpjärvi: A. Employment (full or part-time); Significant; Blueprint Genetics. S. Tuupanen: A. Employment (full or part-time); Significant; Blueprint Genetics. K. Wells: A. Employment (full or parttime); Significant; Blueprint Genetics. L. Sarantaus: A. Employment (full or part-time); Significant; Blueprint Genetics. P. von Nandelstadh: A. Employment (full or part-time); Significant; Blueprint Genetics. K. Merkkiniemi: A. Employment (full or part-time); Significant; Blueprint Genetics. H. Västinsalo: A. Employment (full or part-time);
Significant; Blueprint Genetics. E. Mårtensson: A. Employment (full or part-time); Significant; Blueprint Genetics. R. Perez Carro: A. Employment (full or part-time); Significant; Blueprint Genetics. E. Sankila: A. Employment (full or part-time); Significant; Blueprint Genetics. J. W. Koskenvuo: A. Employment (full or part-time); Significant; Blueprint Genetics. E. Ownership Interest (stock, stock options, patent or other intellectual property); Significant; Blueprint Genetics. S. Myllykangas: A. Employment (full or part-time); Significant; Blueprint Genetics. E. Ownership Interest (stock, stock options, patent or other intellectual property); Significant; Blueprint Genetics. T. Alastalo: A. Employment (full or part-time); Significant; Blueprint Genetics. E. Ownership Interest (stock, stock options, patent or other intellectual property); Significant; Blueprint Genetics.

\section{C15.03}

Insights into the retinal function of ciliopathy proteins gained through zebrafish models

\section{Masek, R. Bachmann-Gagescu*}

\section{University of Zurich-Medical Genetics, Zürich, Switzerland}

Ciliopathies are disorders caused by dysfunction of primary cilia, ubiquitous organelles involved in signal transduction. In retinal photoreceptors (PRs), light sensation occurs in the outer segments (OSs), which represent highly specialized ciliary compartments. Consequently, retinal disease is a common manifestation in ciliopathy patients. Our efforts to elucidate the molecular functions of ciliopathy proteins rely on zebrafish mutants in genes associated with Joubert syndrome, an iconic ciliopathy associated with retinal disease in $25 \%$ of patients. We focus on genes that encode proteins participating in distinct ciliary complexes: $\mathrm{Cc} 2 \mathrm{~d} 2 \mathrm{a}$ at the transition zone, representing the gate to the cilium, or Talpid3/KIAA0586 at the basal body (BB), anchoring the cilium in the cell. While both mutants display decreased visual function, we identify distinct ultra-structural phenotypes indicating specific roles for each protein in primary cilium function. We find that $\mathrm{Cc} 2 \mathrm{~d} 2 \mathrm{a}$ organizes the vesicle fusion machinery at the periciliary margin and provides a docking point for incoming vesicles through a chain of interactions linking CC2D2A to NINL-MICAL3-RAB8. In contrast, Talpid3 is involved in BB docking and ciliogenesis upstream of Rab8a activation. Retina-specific transcriptomics identifies both shared and distinct effects on gene transcription: while transcription of phototransduction genes is decreased in both mutants, likely as a secondary effect, we observe downregulation of the BBSome, a multiprotein complex associated with the ciliopathy Bardet-Biedl syndrome, only in talpid3 mutants, while Nek8 is 
upregulated only in cc2d $2 \mathrm{a}$ mutants. In conclusion, our findings demonstrate how mutations in genes causing the same human disorder result in similar phenotypes through distinct mechanisms.

M. Masek: None. R. Bachmann-Gagescu: None.

\section{C15.04}

Functional characterization of a Xenopus tropicalis knockout and a human cellular model of RCBTB1associated inherited retinal disease shows involvement of RCBTB1 in the cellular response to oxidative stress

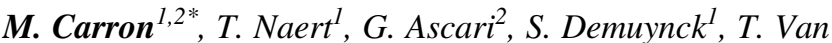
Nieuwenhuyzen $^{1}$, T. Rosseel ${ }^{2}$, D. Priem ${ }^{3,4}$, A. Kremer ${ }^{4}, H$. Van Landeghem $^{l}$, S. Hoogstoel ${ }^{2}$, F. Coppieters ${ }^{2}, C$. Guérin $^{5}$, K. Vleminckx ${ }^{1}$, E. De Baere ${ }^{2}$

${ }^{1}$ Ghent University, Biomedical Molecular Biology, Ghent, Belgium, ${ }^{2}$ Ghent University, Center for Medical Genetics and Dept of Biomolecular Medicine, Ghent, Belgium, ${ }^{3}$ Ghent University, Ghent, Belgium, ${ }^{4}$ VIB-UGent Center for Inflammation Research, Ghent, Belgium, ${ }^{5}$ VIB Bioimaging Core, Ghent, Belgium

Introduction: The function of RCBTB1, recently implicated in syndromic and non-syndromic inherited retinal disease (IRD), remains unknown so far. Patients with biallelic missense variants in RCBTB1 display diverse IRD phenotypes such as retinitis pigmentosa. Here, we tested the hypothesis that RCBTB1 is involved in NRF2-regulated protection against reactive oxygen species in the eye, more specifically in the retinal pigment epithelium (RPE).

Materials and Methods: A Xenopus tropicalis rcbtb1-/knockout (KO) was generated using CRISPR/Cas9 editing. Histology and three-dimensional electron microscopy was performed on retinas of rcbtb1-/- frogs. RNA-seq analysis was performed on RCBTB1-mutated patients' lymphocytes, treated with $\mathrm{H} 2 \mathrm{O} 2$, as well as on embryos from the rcbtb1-/KOs treated with $\mathrm{CdCl} 2$. An RCBTB1 knockdown cell line was generated in ARPE-19 cells and functional assays (flow cytometry, MTT-assay, cell death kinetics) assessed the consequences of RCBTB1 loss-of-function.

Results: Rcbtb1-/- animals showed changes in the RPE, similar to observations in human cases, including loss of apical-basal cell polarity, cuboidal cell morphology, spreading of the pigment granules and vacuolisation. NRF2 downstream targets and several metallothioneins were found to be differentially expressed, both in the KO and cellular models. Functional assays in ARPE-19 cells revealed that RCBTB1 depletion affects cellular responses to external insults of oxidative stress.

Conclusion: We showed that the Xenopus tropicalis rcbtb1-/- KO recapitulates the human IRD phenotype. Both in vivo and in vitro functional data show involvement of RCBTB1 in the cellular response to oxidative stress. This provides insight into the mechanism underlying RCBTB1associated IRD and uncovers potential therapeutic opportunities.

Funding: FWO/1802220N; FWO/1S52818N; BOF15/ GOA/011; BOF20/GOA/023.

M. Carron: None. T. Naert: None. G. Ascari: None. S. Demuynck: None. T. Van Nieuwenhuyzen: None. T. Rosseel: None. D. Priem: None. A. Kremer: None. H. Van Landeghem: None. S. Hoogstoel: None. F. Coppieters: None. C. Guérin: None. K. Vleminckx: None. E. De Baere: None.

\section{C15.05}

Loss of function of RIMS2, a synaptic membrane exocytosis gene, causes a new phenotype characterized by congenital cone-rod synaptic disease associated with neurodevelopmental and pancreatic involvement

S. Mechaussier ${ }^{1 *}$, B. Almoallem ${ }^{2}$, C. Zeitz $^{3}$, K. Van Schil ${ }^{2}$, L. Jeddawi ${ }^{4}, J$. Van Dorpe ${ }^{5}, C$. Condroyer ${ }^{3}$, O. Pelle ${ }^{6}, M$.

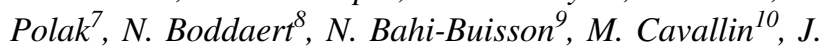
Bacquet $^{11}$, A. Mouallem-Bézière ${ }^{11}$, O. Zambrowski ${ }^{11,12}, \mathrm{~J}$. Sahel $^{3,13,14}$, I. Audo ${ }^{3,14,15}$, J. Kaplan ${ }^{1,11}$, J. Rozet ${ }^{1}$, E. De Baere $^{2}$, I. Perrault ${ }^{1}$

${ }^{1}$ Laboratory of Genetics in Ophthalmology (LGO), INSERM UMR1163, Institute of Genetic Diseases, Imagine and Paris University, Paris, France, ${ }^{2}$ Center for Medical Genetics, Ghent University and Ghent University Hospital, Ghent, Belgium, ${ }^{3}$ Sorbonne Université, INSERM, CNRS, Institut de la Vision, Paris, France, ${ }^{4}$ Pediatric Ophthalmology Division, Dhahran Eye Specialist Hospital, Dharan, Saudi Arabia, ${ }^{5}$ Department of Pathology, Ghent University and Ghent University Hospital, Ghent, Belgium, ${ }^{6}$ Cell Sorting Facility, INSERM UMR1163, Institute of Genetic Diseases, Imagine and Paris University, Paris, France, ${ }^{7}$ Endocrinology, Gynecology and Pediatric Diabetology Department, University Hospital Necker-Enfants Malades, Paris, France, ${ }^{8}$ Department of Pediatric Radiology, Hôpital Necker-Enfants Malades, Paris University, Paris, France, ${ }^{9}$ Pediatric Neurology Department, University Hospital Necker-Enfants Malades, Paris, France, ${ }^{10}$ Laboratory of Embryology and genetics of human malformation, INSERM UMR1 163, Institute of Genetic Diseases, Imagine and Paris University, Paris, France, ${ }^{11}$ Service d'Ophtalmologie, Centre Hospitalier Intercommunal de Créteil (CHIC), Créteil, France, ${ }^{12}$ Ophthalmology Department, University Hospital Necker-Enfants Malades, Paris, France, ${ }^{13}$ Department of Ophthalmology, The University of Pittsburgh School of Medicine, Pittsburgh, PA, United States, ${ }^{14}$ Centre Hospitalier National d'Ophtalmologie des QuinzeVingts, INSERM-DHOS CIC 1423, Paris, France, 
${ }^{15}$ Institute of Ophthalmology, University College of London, London, United Kingdom

Introduction: Anomalies in pre- and post-synaptic transmission of visual information from photoreceptors to bipolar cells are responsible for stationary congenital night blindness (CSNB). Pre-synaptic disorders, characterized by congenital nystagmus and photophobia, night blindness, are known as cone-rod synaptic disorder (CRSD) can be mistaken for Leber congenital amaurosis (LCA). Full-field electroretinogram is crucial for the differential diagnosis, but it can be difficult to perform in young children. Here, we report (i) the identification of biallelic mutations in the RIMS2 gene encoding a pre-synaptic protein not yet associated with human diseases in 3/4 families initially referred for LCA and (ii) the diagnostic adjustment to CRSD with variable associated alteration including autistic behavior and insulinopathy.

Methods: Exome sequencing combined with targeted RIMS2 testing were performed in 46 prescreened patients with CRSD and 94 with LCA. Biallelic RIMS2-mutated cases underwent ophthalmologic, neurologic and metabolic assessment. RIMS2 immunostaining was performed on human retina, brain and pancreas. Nonsense variants were functionally investigated in mammalian cells. Results. Biallelic RIMS2 sequence variants were found in four unrelated families. RIMS2, a protein regulating synaptic membrane exocytosis localizes in the human retinal outer plexiform layer, Purkinje cells and pancreatic islets. Apart from CRSD, neurodevelopmental disease was observed in all cases, and abnormal glucose homeostasis in the eldest patient.?Nonsense variants lead to truncated RIMS2 and decreased insulin secretion in mammalian cells.

Conclusion: This is the first report of syndromic CRSD with neurodevelopmental or pancreatic involvement due to RIMS2 loss-of-function. The disease presentation is consistent with the localization and function of RIMS2.

S. Mechaussier: None. B. Almoallem: None. C. Zeitz: None. K. Van Schil: None. L. Jeddawi: None. J. Van Dorpe: None. C. Condroyer: None. O. Pelle: None. M. Polak: None. N. Boddaert: None. N. Bahi-Buisson: None. M. Cavallin: None. J. Bacquet: None. A. MouallemBézière: None. O. Zambrowski: None. J. Sahel: None. I. Audo: None. J. Kaplan: None. J. Rozet: None. E. De Baere: None. I. Perrault: None.

\section{C15.06}

Genetic and molecular analysis of urinary magnesium concentration in Scottish and Croatian populations

\author{
C. B. Joseph ${ }^{1 *}$, C. M. Stanton ${ }^{1}$, C. Drake ${ }^{1}$, T. S. Boutin ${ }^{1}$, J. \\ F. Wilson ${ }^{2}$, O. Polasek ${ }^{3}$, O. Devuyst ${ }^{4}$, T. Hurd ${ }^{l}, C$. \\ Hayward $^{1}$
}

${ }^{1}$ MRC Human Genetics Unit, Institute of Genetics and Molecular Medicine, University of Edinburgh, Edinburgh, United Kingdom, ${ }^{2}$ Centre for Global Health Research, Usher Institute for Population Health Sciences and Informatics, University of Edinburgh, Edinburgh, United Kingdom, ${ }^{3}$ Split Medical School, Split, Croatia, ${ }^{4}$ Institute of Physiology, University of Zurich, Zurich, Switzerland

Magnesium is the second most abundant bivalent cation in the body and is essential for many cellular processes. Renal magnesium handling plays an important role in maintaining magnesium homeostasis, however the exact biological mechanisms remain unclear. A recent genome-wide association study (GWAS) identified an association between urinary magnesium concentration $(\mathrm{uMg})$ and variants in the ARL15 gene on chromosome 5. ARL15 encodes a GTPbinding protein that regulates the magnesium transporter channel TRPM6, and other proteins involved in magnesium homeostasis in physiologically relevant cell lines. We conducted meta-analyses for urinary magnesium in 11, 617 individuals from Scottish and Croatian populations to determine possible genes involved in these traits. Mass spectrometry analysis was conducted to determine interactors of Arl15 and in-vitro knockout models of Arl15 was established using CRISPR-Cas9. The top SNP associated with $\mathrm{uMg}$ in our meta-analyses lies within a transcription factor binding site in an enhancer region of ARL15. Individuals homozygous for the alternate allele of rs35931 has lower urinary magnesium levels compared to individuals with the homozygous reference allele. Arl15 interacts with magnesium transporters within the distal convoluted tubule segment of the kidneys. Furthermore, we find that homozygous deletion of Arl15 is lethal at the organismal level. We hypothesise that the variant found affects the expression of ARL15, which, in turn modulates magnesium transport by regulating magnesium transporters. We are performing further functional studies to elucidate the role ARL15 plays in magnesium homeostasis both in vitro and in vivo.

C. B. Joseph: None. C. M. Stanton: None. C. Drake: None. T. S. Boutin: None. J. F. Wilson: None. O. Polasek: None. O. Devuyst: None. T. Hurd: None. C. Hayward: None.

\section{C15.08}

RNA-seq approach to detect splicing alterations in primary ciliary dyskinesia and non-CF bronchiectasis

\author{
J. Legebeke ${ }^{1 *}$, G. Wheway ${ }^{l}, H$. Wai $^{l}$, W. Walker ${ }^{1,2}$, J. S. \\ Lucas $^{1,2}$, D. Baralle ${ }^{1,2}$ \\ ${ }^{1}$ University of Southampton, Southampton, United King- \\ dom, ${ }^{2}$ University Hospital Southampton, Southampton, \\ United Kingdom
}


Mutations that cause PCD have been reported in $>40$ genes. Most cases are recessive, caused by biallelic changes in these genes. These account for around $70 \%$ of cases, suggesting that additional genes, and non-coding or synonymous changes in known genes, remain to be identified. Non-CF bronchiectasis is a heterogeneous group of disorders and adults may need to be investigated for PCD. Here we present an optimised pipeline for RNA analysis from nasal brushings taken from the inferior turbinate of patients with suspected PCD or non-CF bronchiectasis. We present analysis pipelines to investigate differences in gene expression, alternative splicing and the identification of deleterious splicing events. We developed gene-neutral and gene-specific analysis protocols, from both nasal brushings stored in RNA-later and cells from nasal brushings cultured at the air-liquid interface (ALI). We present this RNA-seq approach as a particularly useful diagnostic tool to investigate patients in whom a single pathogenic variant has been identified in a known PCD gene and where we suspect an alteration in splicing of the same gene due to a second intronic or synonymous / non-synonymous exonic variant. This represents a step towards using RNA analysis for increasing diagnostic yield in rare recessive respiratory disease. JL, DB and HT are supported by a NIHR Research Professorship to DB (RP- 2016-07-011). JL is further supported by Southampton NIHR BRC. GW is funded by a Wellcome Trust Seed Award in Science (Grant No. 204378/ Z/16/Z) and a University of Southampton Faculty of Medicine Research Management Committee research grant.

G. Wheway: None. H. Wai: None. W. Walker: None. J. S. Lucas: None. D. Baralle: None.

\section{C15.09}

Combined exome/genome sequencing with in-house variant data repository mining confirm USP53 as a causal gene for intrahepatic cholestasis

S. Alawbathani ${ }^{1 *}, M$. Rocha $^{l}, H$. Arshad Cheema ${ }^{2}, R$. Boustany ${ }^{3}$, S. Nampoothiri, A. Alswaid ${ }^{5}$, N. El Koofy ${ }^{6}$, V.

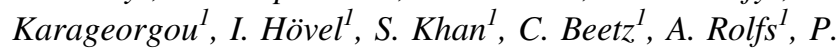
Bauer $^{l}$, A. M. Bertoli-Avella ${ }^{l}$

${ }^{1}$ Centogene AG, Rostock, Germany, ${ }^{2}$ Children's Hospital and Institute of Child Health, Lahore, Pakistan, ${ }^{3}$ AUBMC American University of Beirut Medical Center, Beirut, Lebanon, ${ }^{4}$ Amrita Institute of Medical Sciences, Kerala, India, ${ }^{5}$ King Abdulaziz Medical City, Riadh, Saudi Arabia, ${ }^{6}$ Abu El Reesh Children's Hospital, Cairo, Egypt

Cholestatic liver disease is a significant cause of morbidity and mortality in the paediatric population, starting in the early postnatal life. Severity is variable, from benign idiopathic neonatal hepatitis to intrahepatic cholestasis leading to liver failure and transplant. There are several genes described for different genetic diseases that lead to cholestasis. USP53 has been recently suggested as a candidate gene for paediatric intrahepatic cholestasis based on one family with two affected siblings. Interestingly, USP53 interacts with tight junction protein 2 (TJP2) for which pathogenic variants are known to cause progressive familial intrahepatic cholestasis. To date, the USP53 gene has not been linked to an OMIM phenotype. By combining exome/ genome sequencing and mining our in-house variant database we identified five patients from unrelated families. All affected individuals display a similar phenotype, mainly including early onset intrahepatic cholestasis. Moreover, one family showed hearing loss as an additional phenotype. All patients were homozygotes for different loss-of-function variants in the USP53 gene. USP53 (ubiquitin specific peptidase 53) is a tight junction-associated protein that is known to involve in the survival of auditory hair cells and hearing. Our results confirm USP53 as a novel gene for autosomal recessive intrahepatic cholestasis. Additionally, we demonstrate that combined exome/genome sequencing and variant database mining is an effective approach to discover and confirm novel genes related to human diseases. This accelerate the process of gene discovery and ultimately shortens the diagnostic odyssey in rare genetic disorders.

S. Alawbathani: A. Employment (full or part-time); Significant; Centogene AG, Rostock, Germany. M. Rocha: A. Employment (full or part-time); Significant; Centogene AG, Rostock, Germany. H. Arshad Cheema: None. R. Boustany: None. S. Nampoothiri: None. A. Alswaid: None. N. El Koofy: None. V. Karageorgou: A. Employment (full or part-time); Significant; Centogene AG, Rostock, Germany. I. Hövel: A. Employment (full or part-time); Significant; Centogene AG, Rostock, Germany. S. Khan: A. Employment (full or part-time); Significant; Centogene AG, Rostock, Germany. C. Beetz: A. Employment (full or part-time); Significant; Centogene AG, Rostock, Germany. A. Rolfs: A. Employment (full or part-time); Significant; Centogene AG, Rostock, Germany. P. Bauer: A. Employment (full or part-time); Significant; Centogene AG, Rostock, Germany. A. M. Bertoli-Avella: A. Employment (full or part-time); Significant; Centogene AG, Rostock, Germany.

\section{C15.10}

Elucidating the molecular mechanism underlying laterality defects

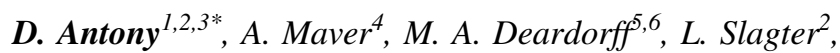
K. Wu ${ }^{2}$, Z. Bakey ${ }^{1,2}$, E. Gulec ${ }^{7}$, A. Gezdirici ${ }^{7}$, H. Brunner ${ }^{2,3}$, M. Schmidts ${ }^{1,2,3}$ 
${ }^{1}$ Center for Pediatrics and Adolescent Medicine, University Hospital Freiburg, Freiburg, Germany, ${ }^{2}$ Genome Research Division, Department of Human Genetics, Radboud UMC, Nijmegen, Netherlands, ${ }^{3}$ Radboud Institute of Molecular Life Sciences, Nijmegen, Netherlands, ${ }^{4}$ Centre for Mendelian Genomics, Clinical Institute of Medical Genetics, UMC Ljubljana, Ljubljana, Slovenia, ${ }^{5}$ Department of Pediatrics and Pathology, The Perelman School of Medicine, University of Pennsylvania, Pennsylvania, PA, United States, ${ }^{6}$ Laboratory Medicine, The Perelman School of Medicine, University of Pennsylvania, Pennsylvania, PA, United States, ${ }^{7}$ University of Health Sciences, Kanuni Sultan Suleyman training and research Hospital, Department of Medical Genetics, Istanbul, Turkey

Introduction: Laterality defects are rare developmental disorders, occurring isolated or as part of complex syndromes. Underlying cause is frequently dysfunction of motile cilia causing Primary Ciliary Dyskinesia (PCD) and rarely, non-motile cilia defects or non-ciliary causes. In addition to randomization of the left right body axis, PCD is characterized by frequent respiratory infections and infertility.

Methods: We used Whole Exome Sequencing (WES) to delineate the underlying molecular cause in 37 mainly consanguineous families with laterality defects.

Results: We identified causative variants in $32 \%$ with majority of mutations detected in genes previously associated with PCD, including 2 small homozygous CNVs. Variants in non PCD genes, PKD111 and GJA1 were also detected. We further identified homozygous null mutations in two genes not previously associated with human disease, MNS1 and DNAH9, both in cases with situs inversus but little or no respiratory symptoms. Gene matcher database revealed two additional families with biallelic DNAH9 mutation and immunofluorescence analysis revealed complete absence of DNAH9 from respiratory cilia of one affected while DNAH9 localises to the distal half of cilia in controls. Further, we found direct interaction of DNAH9 with the ODA docking complex protein $\mathrm{CCDC} 114$ using $\mathrm{Y} 2 \mathrm{H}$ screening also interaction with DNAH5 and DNAI2 using coImmunoprecipitation.

Conclusion: We could only solve a third of the laterality cases genetically using WES, pointing towards non coding variations, genetic mosaicism or epigenetic effects while we were able to identify two novel human disease causing genes, MNS1 and DNAH9, both causing laterality defects with no or little respiratory disease. ERC: TreatCilia

D. Antony: None. A. Maver: None. M. A. Deardorff: None. L. Slagter: None. K. Wu: None. Z. Bakey: None. E. Gulec: None. A. Gezdirici: None. H. Brunner: None. M. Schmidts: None.

\section{C15.11}

Biallelic missense variant in SVBP causes impaired detyrosination of tubulin leading to complex hereditary spastic paraplegia without microcephaly

E. Verdura ${ }^{1,2^{*}}, N$. Launay $^{l, 2}$, G. Fernandez Garcia de Eulate $^{3,4,5}$, J. Ondaro ${ }^{4,5}$, A. Schlüter ${ }^{1,2}$, M. Ruiz ${ }^{1,2}, S$. Fourcade $^{1,2}$, A. López de Munain ${ }^{3,4,5}$, A. Pujol ${ }^{1,2}$

${ }^{I}$ Neurometabolic Disease Lab, Institute of Neuropathology, IDIBELL, L'Hospitalet de Llobregat, Barcelona, Spain, ${ }^{2}$ Center for Biomedical Research on Rare Diseases (CIBERER U759), Ministry of Science, Innovation and University, Madrid, Spain, ${ }^{3}$ Department of Neurology, Hospital Universitario Donostia, San Sebastián, Spain, ${ }^{4}$ Department of Neurosciences, Instituto Biodonostia, San Sebastián, Spain, ${ }^{5}$ Department of Neurosciences, School of Medicine and Nursery, University of the Basque Country, UPV-EHU, San Sebastián, Spain

Introduction: Reversible C-Terminal detyrosination of alpha-tubulin is crucial for microtubule organization, and is strongly linked to neuronal differentiation. VASH1 and VASH2, in complex with SVBP, have recently been identified as responsible for intracellular tubulin detyrosination activity. Biallelic loss-of-function mutations in SVBP have much recently been described in patients showing intellectual disability, microcephaly, ataxia and hypotonia.

Methods: All patients underwent accurate clinical evaluation and provided informed consent. Whole-exome sequencing (WES) was performed using patients' DNA samples, and segregation in relatives was confirmed by Sanger sequencing. Primary fibroblasts were generated from patients' skin biopsies, and cultured cells were incubated with paclitaxel to analyze detyrosinated tubulin levels by immunofluorescence and western blot. cDNA of VASH1 and wild-type/mutated forms of SVBP were cloned into a pCMV-Tag4A vector, and then cotransfected into HeLa cells for Western Blot analysis.

Results: We report two families with multiple patients harboring an homozygous missense variant in SVBP (p. Leu49Pro). Affected individuals show spastic paraplegia, peripheral neuropathy, verbal apraxia and intellectual disability, in absence of microcephaly, broadening the previously reported phenotype. In vitro experiments in patients' fibroblasts demonstrate a significant loss of VASH1/2 detyrosination activity and decreased SVBP levels. Cotransfection studies show that SVBP p.Leu49Pro variant reduces both VASH1 and SVBP soluble quantities or their stability.

Conclusions: Our results expand the phenotypic spectrum of SVBP mutations to complex spastic paraplegia without microcephaly, and confirm for the first time that a 
detyrosination defect is observed in patients' cell lines, enabling functional testing of SVBP variants and extended pathogenetic studies.

E. Verdura: None. N. Launay: None. G. Fernandez Garcia de Eulate: None. J. Ondaro: None. A. Schlüter: None. M. Ruiz: None. S. Fourcade: None. A. López de Munain: None. A. Pujol: None.

\section{C15.13}

Understanding the mechanism of RNF216-mediated neurodegeneration: genetic, proteomic, neuropathological and cellular approach

\section{S. Roy $^{1,2 *}$, P. Ervilha Pereira ${ }^{1,2}, N$. Schuermans ${ }^{1,2}, A$. Sieben $^{3,4}$, F. Thery ${ }^{5,6}$, E. Mondragon Rezola ${ }^{7}$, E.

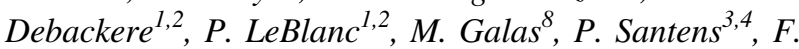 Impens $^{5,6}$, E. Bogaert ${ }^{1,2}$, B. Dermaut ${ }^{1,2}$}

${ }^{1}$ Center for Medical Genetics, Ghent University Hospital, Gent, Belgium, ${ }^{2}$ Department of Biomolecular Medicine, Faculty of Medicine and Health Sciences, Ghent University, Ghent, Belgium, ${ }^{3}$ Department of Neurology, Ghent University Hospital, Gent, Belgium, ${ }^{4}$ Department of Head and Skin, Faculty of Medicine and Health Sciences, Ghent University, Ghent, Belgium, ${ }^{5}$ Department of Biomolecular Medicine, Faculty of Medicine and Health Sciences, Ghent University, Gent, Belgium, ${ }^{6}$ Center for Medical Biotechnology, VIB Ghent, Ghent, Belgium, ${ }^{7}$ Department of Neurology, University Hospital Donostia, Basque Country University, Donostia, Spain, ${ }^{8}$ Inserm, UMRS1172, JPARC, Alzheimer \& Tauopathies, Lille, France

Introduction: Neurodegenerative diseases frequently result from mutations in ubiquitin-signaling genes. Our group was among the first to report bi-allelic mutations in RNF216, an E3 ubiquitin ligase, as the genetic cause of Gordon Holmes syndrome (GHS), an adult-onset rare neurodegenerative disorder characterized by ataxia, dementia and hypogonadotropic hypogonadism or Huntington-like disorder (HDL). To date, $>10$ mutations in RNF216 have been reported not only from the catalytic active site but also from $\mathrm{N}$-terminal part of the protein.

Objective: We intend to unravel the mechanisms by which RNF216 mutations lead to GHS and HDL. We aim to identify novel RNF216 mutations for genotypephenotype correlation studies, identify protein composition of the pathological intranuclear inclusions, and understand the cellular and biochemical function of the RNF216 protein.

Methods: Frozen and fixed brain material from patient were used for generalized proteomics and neuropathological studies and patient fibroblast and Hela cells were used for cellular studies.
Results: We describe a Spanish patient with HDL having 2 novel compound heterozygous missense mutations in RNF216. The study of brain material from one of the two reported Belgian families demonstrated ubiquitin- and p62positive intranuclear inclusions, negative for Tau, ?-synuclein and TDP-43. Proteomics generated a list of candidate genes whose histopathological examination is currently ongoing. Hela cells transfected with wildtype and mutant RNF216 constructs revealed RNF216 and ubiquitin positive intranuclear speckle-like bodies.

Conclusion: The identity of intranuclear bodies should be further analyzed hoping to reveal molecular intricacies linked to this neurodegenerative disease. Odysseus Type-I (G0H8318N) FWO grant, Starting grant (01N10319) Ghent University

S. Roy: None. P. Ervilha Pereira: None. N. Schuermans: None. A. Sieben: None. F. Thery: None. E. Mondragon Rezola: None. E. Debackere: None. P. LeBlanc: None. M. Galas: None. P. Santens: None. F. Impens: None. E. Bogaert: None. B. Dermaut: None.

\section{C15.14}

The genotype-phenotype correlation of intragenic RBFOX1 deletions.

\section{A. Paczkowska*, M. Geremek, B. Nowakowska}

\section{Institute of Mother and Child, Warsaw, Poland}

The RBFOX1 gene encodes a protein that regulates premRNA splicing in developmental genes expressed in muscle, heart and neuronal cells. Intragenic deletions of RBFOX1 gene have been identified as susceptibility loci and reported in patients with aggressive behavior, conduct disorder, epilepsy and autism spectrum disorder. Despite of the documented correlation with neurodevelopmental disorders, the deletions of RBFOX1 gene are often inherited from the healthy parents, therefore the clinical interpretation of CNVs within this gene is still unclear. We applied oligonucleotide microarrays (OGT) in a cohort of 9430 patients with neurodevelopment disorders and their healthy relatives. The analyses of genomes were performed using genome-wide array to accurate detection of copy number variation at the exon level in RBFOX1 gene. Chromosomal analysis revealed intragenic RBFOX1 deletions in 36 patients. The differential distribution across the gene between patients with neurodevelopmental disorders and the control group allowed to distinguished evidently pathogenic areas within the gene. Deletions of exones 1-2 and 13-14 have high penetrance, and are mainly associated with conduct disorder and autism spectrum disorder. While the middle part of the gene and all intronic CNVS were observed with higher frequency in the control group, 
suggesting their benign impact on the phenotype. The work was supported by MINDDS - CA COST Action CA16210 Maximising Impact of research in NeuroDevelopmental DisorderS

M. A. Paczkowska: None. M. Geremek: None. B. Nowakowska: None.

\section{C15.15}

Mitochondrial dysfunction and oxidative stress contribute to the phenotype of FOXP1 deficiency

\section{J. Wang ${ }^{*}$, H. Fröhlich, G. A. Rappold \\ Institut für Humangenetik, Heidelberg, Germany}

Patients with FOXP1 haploinsufficiency present intellectual disability, autism spectrum disorder, severe speech and language impairments as well as motor dysfunction. To unravel the pathological mechanism underlying FOXP1 syndrome, we analysed Foxp1+/- mice, which closely reflect FOXP1 haploinsufficiency. We can show that Foxp1+/- animals exhibit behavioural deficits such as reduced neonatal ultrasonic vocalization, hyperactivity, anxiety and alterations in spatial navigation and memory. Furthermore, they manifest decreased exercise capacity and endurance. We also found that Foxp1+/- animals display a reduced striatal expression of proteins involved in mitochondrial biogenesis and dynamics (Pgc-1?, Tfam, Opa1 and Drp1), suggesting that mitochondrial dysfunction may contribute to the symptoms. To address this issue in more detail, we investigated mitochondria in WT and Foxp1+/- animals. We demonstrate that Foxp1+/- striatal neurons display a disrupted mitochondrial membrane potential. In addition, we detected an increased abundance of reactive oxygen species, increased cytochrome $\mathrm{C}$ release as well as lipid peroxidation in the Foxp1+/- striatum. In summary, our results indicate mitochondrial dysfunction in murine Foxp1 deficiency, suggesting that mitochondrial dysfunction and increased oxidative stress may also contribute to the deficits in FOXP1 Syndrome.

H. Fröhlich: None. G. A. Rappold: None.

\section{C16 The Interplay of Law, Policy and Genomics}

\section{C16.1}

Regulating Preimplantation Genetic Diagnosis: A comparison of international policy trends and perspectives

\section{R. Isasi ${ }^{*}$, M. E. Ginoza}

Miller School of Medicine, University of Miami, Miami, FL, United States
Preimplantation genetic diagnosis (PGD) is a reproductive technology which allows prospective parents to select their future offspring based on genetic characteristics. During the last decade, its regulation has followed mostly a linear path, with incremental changes driven by scientific advances or by court driven alterations. But also, shift has been prompted by greater societal uptake. No longer considered an experimental practice, stakeholders have challenged the status quo, recognizing that both technological and policy developments do not take place in a moral vacuum. They have done so under the flag of reproductive freedom, equitable access and welfare considerations, amongst other ethical concerns. Despite the passage of time, the ubiquitous presence of eugenics seems to constantly cast a shadow over technologies that open the door for selecting the genetic characteristics of a human offspring. For this reason, we can predict that despite greater societal acceptance, widening its permissibility criteria will remain contested and a matter of continue social and policy debate. In this presentation, we examine variations in policy approaches. We draw on a representative sample of 19 countries (Australia, Austria, Belgium, Brazil, Canada, China, France, Germany, India, Israel, Italy, Japan, Mexico, Netherlands, Singapore, South Korea, Switzerland, United Kingdom, and the United States) to provide a global landscape of the spectrum of policy and legislative approaches. We also explore central socio-ethical and policy issues and contentious applications as for instance, permissibility criteria (e.g. medical necessity), non-medical sex selection and reproductive tourism. Finally, we further outline genetic counseling requirements across policy approaches.

R. Isasi: None. M. E. Ginoza: None.

\section{C16.2}

Regulation of international direct-to-participant (DTP) genomic research: results and recommendations from a 31-country study

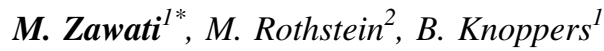 \\ ${ }^{1}$ McGill University, Montreal, QC, Canada, ${ }^{2}$ University of Louisville, Louisville, KY, United States}

International direct-to-participant (DTP) research promises to greatly increase the number and diversity of participants for research on genetic disorders. This phenomenon, in which participants recruited online supply biosamples or provide access to electronic health records, may be thwarted by prohibitions on research without ethics review in each participating country. Though global recruitment is a promising research strategy, there is an urgent need to understand the international regulatory landscape to foster a more global DTP genomics research ecosystem. In this 
presentation, we set out to determine how DTP genomics research is regulated in 31 countries. We engaged with local experts in 31 countries, each of whom were asked to prepare a report of 2,000 words in response to a 10-item questionnaire on the regulation of DTP research. These reports represent the first systematic effort to assess the legal environment for international DTP genomic research. We will describe the results of this work, including the state of regulation of international genomic research practices. We found, for example, that 17/31 experts reported it would be unlawful for an international researcher to conduct DTP research without local ethics approval. We will conclude by outlining our team's recommendations on international DTP genomic research, elaborating on themes of ethics equivalence and the importance of incorporating cultural considerations in policy development. While there are significant legal barriers to expanding DTP genomics research internationally, sound policy will maximize its potential.

Funding information: National Institute of Health (NIH), National Human Genome Research Institute (NHGRI), Grant No. 5R01HG009914-02.

M. Zawati: None. M. Rothstein: None. B. Knoppers: None.

\section{C16.3}

Genomic sequencing capacity, data retention and personal access to raw data in Europe

S. Narayanasamy ${ }^{1 *}$, V. Markinal ${ }^{l}$, A. Thorogood ${ }^{2}, A$. Blazkova $^{1}$, M. Shabani ${ }^{3}$, B. M. Knoppers ${ }^{2}$, B. Prainsack ${ }^{4}, R$. Koesters ${ }^{l}$

${ }^{1}$ Megeno S.A., Esch-sur-Alzette, Luxembourg, ${ }^{2}$ Centre of Genomics and Policy, McGill University, Montreal, QC, Canada, ${ }^{3}$ Metamedic, Faculty of Law and Criminology, Ghent University, Ghent, Belgium, ${ }^{4}$ Department of Political Science, University of Vienna, Vienna, Austria

Whole genome/exome sequencing (WGS/WES) has become widely adopted in research and, more recently, in clinical settings. Many hope that the information obtained from interpretation of this data will have medical benefits for patients and - in some cases - also their biological relatives. Because of the manifold possibilities to reuse genomic data, enabling sequenced individuals to access their own raw (uninterpreted) genomic data is a highly debated issue. This paper reports some of the first empirical findings on personal genome access policies and practices. We interviewed 39 respondents, working at 33 institutions in 21 countries across Europe. These sequencing institutions generate massive amounts of WGS/WES data and represent varying organisational structures and operational models. Taken together, in total, these institutions have sequenced
317,259 genomes and exomes to date. Most of the sequencing institutions reported that they are able to store raw genomic data in compliance with various national regulations, although there was a lack of standardisation of storage formats. Interviewees from 12 of the 33 institutions included in our study reported that they had received requests for personal access to raw genomic data from sequenced individuals. In the absence of policies on how to process such requests, these were decided on an ad hoc basis; in the end, at least 28 requests were granted, while there were no reports of requests being rejected. Given the rights, interests, and liabilities at stake, it is essential that sequencing institutions adopt clear policies and processes for raw genomic data retention and personal access.

S. Narayanasamy: A. Employment (full or part-time); Significant; Megeno S. A. V. Markina: A. Employment (full or part-time); Significant; Megeno S. A. A. Thorogood: F. Consultant/Advisory Board; Modest; Megeno S. A. A. Blazkova: A. Employment (full or part-time); Significant; Megeno S.A. M. Shabani: None. B. M. Knoppers: None. B. Prainsack: None. R. Koesters: A. Employment (full or parttime); Significant; Megeno S.A.

\section{C16.4}

The genome as individual property or common good. Conceptualization of the genome within a citizen forum

\section{Mayeur*, W. Van Hoof}

\section{Sciensano, Anderlecht, Belgium}

In recent years, policymakers and experts showed particular interest in public engagement in genomics. However, most projects focus on expressed values, without exploring the concepts behind them. To understand which uses of genomic information citizens support, one should first examine their conceptualization of the human genome and then explore the values they assign to it. We organized a citizen forum on the ethical, legal and societal issues surrounding the use of genomic information in healthcare. During three weekends, a panel of 32 citizens, informed by experts of different backgrounds, debated and reflected with the final aim of producing political recommendations for the ministry of public health. An inductive thematic analysis of their discussions shows that citizens view the genome as the most intimate part of individuals, that warrants protection to avoid breach of privacy. Additionally, citizens claim that humans are more than their genetic makeup: they are socially, culturally, emotionally, and psychologically complex, and should be treated and considered accordingly. This is why citizens vehemently oppose genetic categorization and discrimination. Finally, a fundamental tension remains between two conceptions of the genome - the individual's property versus common good - 
with consequences on sensitive issues such as commercialization, access to and sharing of data, individual responsibility and autonomy. Citizens' conceptions of the genome inform such different norms and values that it is important for policymakers and experts to appreciate them to be able to inform citizens and engage with them on genomics. Innovative Partnership for Action Against Cancer funded this study.

C. Mayeur: None. W. Van Hoof: None.

\section{C17 New Therapeutic Approaches}

\section{C17.1}

An innovative dual-reporter cell line to identify protein level modulators in drug-repositioning for rare genetic diseases: a proof-of-principle in Autosomal Dominant LeukoDystrophy (ADLD)

E. Giorgio ${ }^{1 *}$, E. Pesce ${ }^{2}$, E. Pozzi $i^{1}$, E. Sondo ${ }^{2}$, M. Lorenzati $^{3}$, M. Ferrero ${ }^{1}$, G. Borrelli, E. Della Sala ${ }^{l}, A$.

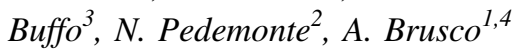

${ }^{1}$ University of Torino-Dep Medical Sciences, Torino, Italy, ${ }^{2}$ IRCCS Istituto Giannina Gaslini-UOC Genetica Medica, Genova, Italy, ${ }^{3}$ University of Torino-Dep. Neuroscience Rita Levi Montalcini and Neuroscience Institute Cavalieri Ottolenghi, Torino, Italy, ${ }^{4}$ Città della Salute e della Scienza University Hospital-Medical Genetics Unit, Torino, Italy

Rare genetic diseases affect a small number of patients each, and thus are of limited interest to the biopharmaceutical industry. Since the vast majority still have no treatment options, a cost-effective strategy is the repurposing of existing drugs as therapies for new disorders. The success of drugrepositioning approaches is strongly related to the optimization of an appropriate reporter cell line suitable for high throughput screening (HTS). Here, we report the development and optimization of a stable cell line constitutively expressing an innovative dual-reporter IRES-containing bicistronic vector allowing the rapid and efficient identification of modulators acting at protein level. Drugs restoring physiological protein levels represent a promising therapeutic strategy for genetic disorders associated with genomic deletions/duplications. An instructive example is Autosomal Dominant LeukoDystrophy (ADLD), a rare disease caused by LMNB1 accumulation due to gene duplication or to a regulatory alteration. We have screened by HTS 400 FDA-approved drugs and we have identified a positive hit. This drug (name reserved) has been validated in 5 different cell lines confirming its ability to specifically reduce LMNB1 levels (30\%$80 \%$ of reduction). Finally, the treatment of ADLD fibroblasts showed a decrease of about $50 \%$ of cytoplasmic LMNB1, supporting the therapeutic value of our molecule. Our results pave the way towards a clinical trial for ADLD following the "compassionate use" practice. Finally, our strategy using a stable dual-reporter cell line can be exploited for identifying effective drugs for other genetic and multifactorial disorders in which the modulation of a protein represents a promising therapeutic option.

E. Giorgio: None. E. Pesce: None. E. Pozzi: None. E. Sondo: None. M. Lorenzati: None. M. Ferrero: None. G. Borrelli: None. E. Della Sala: None. A. Buffo: None. N. Pedemonte: None. A. Brusco: None.

\section{C17.2}

Low-dose infigratinib, an oral selective fibroblast growth factor receptor (FGFR) tyrosine kinase inhibitor (TKI), demonstrates activity in preclinical models of achondroplasia (ACH)

\section{B. Demuynck ${ }^{1 *}, J$. Flipo $^{l}$, G. $L i^{2}$, C. Dambkowski ${ }^{2}, L$. Legeai-Mallet ${ }^{l}$}

${ }^{1}$ Université de Paris, Institut Imagine, Laboratory of Molecular and Physiopathological Bases of Osteochondrodysplasia, INSERM, Paris, France, ${ }^{2}$ QED Therapeutics Inc., San Francisco, CA, United States

Background: $F G F R 3$ gain-of-function mutations play a crucial role in $\mathrm{ACH}$, thanatophoric dysplasia (TD), and hypochondroplasia. Therapies blocking the MAP kinase (MAPK) pathway, such as C-type natriuretic peptide (CNP) analogues, are in development; however, FGFR3 can also promote chondrocyte proliferation via alternate pathways (e.g. STAT1). We tested the hypothesis that the FGFR1-3 selective oral TKI infigratinib (BGJ398) is more efficacious than a CNP analog due to inhibition of both MAPK and STAT1 in preclinical models of ACH carrying FGFR3 gain-of-function mutations.

Methods: Human TD chondrocytes were incubated with/ without infigratinib or vosoritide, and total/phospho-ERK1/ 2 levels were measured. A mouse model $\left(\mathrm{Fgfr}^{\mathrm{Y367C/+}}\right)$ mimicking ACH received subcutaneous injections of infigratinib or vehicle control daily $(0.2,0.5 \mathrm{mg} / \mathrm{kg} /$ day $)$ or every 3 days $(1 \mathrm{mg} / \mathrm{kg})$ for 15 days.

Results: In vitro, $10^{-10} \mathrm{M}$ infigratinib led to significant inhibition of ERK1/2 phosphorylation $(\mathrm{p}<0.05)$ in TD chondrocytes, while vosoritide treatment had significant activity only at concentrations $>10^{-5} \mathrm{M}$. In vivo, significant improvement was observed in upper limbs (humerus $+7 \%$, ulna $+11 \%$ ), lower limbs (femur $+11 \%$, tibia $+16 \%$ ), foramen magnum length and organization of growth plate structure in mice treated with infigratinib (daily and intermittent injections). Infigratinib activity was dose dependent with no apparent toxicities.

Conclusions: Infigratinib has superior activity over vosoritide in vitro, suggesting that inhibition of multiple 
pathways downstream of FGFR3 may lead to better efficacy. Results in vivo indicate that clinically relevant doses of infigratinib significantly promote growth. Preclinical data support development of infigratinib in $\mathrm{ACH}$, with a clinical study launching this year.

B. Demuynck: None. J. Flipo: None. G. Li: A. Employment (full or part-time); Significant; QED Therapeutics Inc. E. Ownership Interest (stock, stock options, patent or other intellectual property); Significant; QED Therapeutics Inc. C. Dambkowski: A. Employment (full or part-time); Significant; QED Therapeutics Inc. E. Ownership Interest (stock, stock options, patent or other intellectual property); Significant; BridgeBio Pharma, Novonate, Origin Biosciences. L. Legeai-Mallet: None.

\section{C17.3}

CRISPR/Cas9 gene editing approach completely repaired the causative COL4A5 10bp deletion in a naturally occurring dog model of Alport Syndrome.

S. Daga ${ }^{1 *}$, K. Capitani ${ }^{2,3}$, F. Donati ${ }^{2}$, G. Beligni ${ }^{l}$, S. Croci ${ }^{l}$, F. Valentino ${ }^{1}$, C. Fallerini ${ }^{1}$, R. Tita ${ }^{4}$, C. Rivera ${ }^{5}$, S. Clark ${ }^{5}$, M. Mencarelli ${ }^{4}$, M. Baldassarri ${ }^{4}$, E. Benetti $i^{1}, S$. Furini $^{3}$, E.

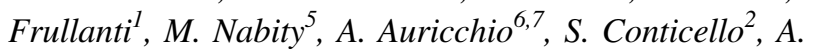
Renieri $^{1,4}$, A. Pinto $^{4}$

${ }^{1}$ Medical Genetics, University of Siena, Siena, Italy, ${ }^{2}$ Core Research Laboratory - ISPRO, Florence, Italy, ${ }^{3}$ Department of Medical Biotechnologies, University of Siena, Siena, Italy, ${ }^{4}$ Genetica Medica, Azienda Ospedaliera Universitaria Senese, Siena, Italy, ${ }^{5}$ Department of Veterinary Pathobiology, Texas A\&M University College of Veterinary Medicine and Biomedical Sciences, College Station, TX, United States, ${ }^{6}$ Telethon Institute of Genetics and Medicine (TIGEM), Pozzuoli, Italy, ${ }^{7}$ Department of Advanced Biomedicine, Federico II University, Naples, Italy

Alport Syndrome (AS) is a genetic disorder characterized by clinicopathologic alterations ranging from GBM abnormalities up to ESRD. Extensively reported mutations in the COLAA3/A4/A5 genes are causative of both the autosomal and the X-linked forms. Although great pharmacological advances have been achieved in the past years, unfortunately no definitive therapy is currently available. Podocytes, terminally differentiated cells, produce a unique collagen in the GBM. We have previously demonstrated that podocytelineage cells can be isolated from AS patients' urine, and we have proven the efficiency of a bi-plasmid (AAV)2CRISPR/Cas9 approach to restore the wild-type genotype in-vitro. Two mutations, namely the COL4A5 p.G624D and the COL4A3 p.G526E, mimicking XLAS and ADAS forms respectively, were effectively corrected with an HDR percentage greater than $44 \%$ and undesired in/dels lower than $12 \%$. Here we show that, moving to a preclinical approach, it is possible to correct the causative COLAA5Ex9-10bp-deletion in a naturally occurring dog model, using a macro-500bp-DNA-Donor. The target region, in $80 \%$ of all treated cells, was corrected without in/dels in the neighboring sites. A silent mutation, inserted as a tag on the PAM, was used to monitor the correction and to protect the DNA from a new spCas9 cut. Based on AAV2/GFP biodistribution, in-vivo infection with (AAV)2-CRISPR/Cas9 will be performed at 2-3 months of age using single carotid artery catheterization to target both renal arteries. This patented approach, providing the proof-of-principle for a revolutionary AS gene therapy, can open up the possibility to novel clinical trials, based on personalized strategy.

Italian patent application n102018000020230

S. Daga: None. K. Capitani: None. F. Donati: None. G. Beligni: None. S. Croci: None. F. Valentino: None. C. Fallerini: None. R. Tita: None. C. Rivera: None. S. Clark: None. M. Mencarelli: None. M. Baldassarri: None. E. Benetti: None. S. Furini: None. E. Frullanti: None. M. Nabity: None. A. Auricchio: None. S. Conticello: None. A. Renieri: None. A. Pinto: None.

\section{C17.4}

Single-intrathecal delivery of a new AAV9-mediated gene therapy vector provides long-term safe expression of frataxin and prevents neurological and cardiac deficits, neurodegeneration and iron deposition in a Friedreich's Ataxia mouse model

E. Balagué ${ }^{1 *}$, D. Cota-González ${ }^{l}, K$. Adrián-Campbell ${ }^{1}, B$. García-Lareu ${ }^{2}$, A. Bosch ${ }^{2}$, J. Coll-Cantî ${ }^{3}$, M. Chillón ${ }^{4}, I$. Sánchez ${ }^{1,5}$, A. Matilla-Dueñas ${ }^{1,5}$

${ }^{1}$ Translational and Functional Neurogenetics Unit, Department of Neuroscience, Germans Trias Research Institute (IGTP), Universitat Autònoma de Barcelona-Campus Can Ruti, Badalona, Spain, ${ }^{2}$ Institut de Neurociències (INc), Department of Biochemistry and Molecular Biology, Universitat Autònoma Barcelona 5th level, Edifici $H$, Institut Neurociencies, Campus UAB, Bellaterra, Spain, ${ }^{3}$ Unitat de Neuromuscular, Hospital Universitari Germans Trias i Pujol, Badalona, Spain, ${ }^{4}$ Institució Catalana de Recerca i Estudis Avançats (ICREA), Vall d'Hebron Institut de Recerca, Hospital Universitari Vall d'Hebron, Vector Production Unit (UPV) and Universitat Autònoma Barcelona, Barcelona, Spain, ${ }^{5}$ Functional Biology and Experimental Therapeutics lab, Translational and Functional Neurogenetics Unit, Department of Neuroscience, Germans Trias Research Institute (IGTP), Universitat Autònoma de Barcelona-Campus Can Ruti, Badalona, Spain 
Introduction: Friedreich's ataxia (FRDA) is a recessively inherited neurodegenerative disease caused by frataxin (FXN) deficiency. FRDA is characterised by progressive ataxia, sensory loss and hypertrophic cardiomyopathy. Neurodegeneration is identified in DRG neurons, peripheral sensory nerves, the posterior column, and cerebellar dentate nucleus. To date, proof-of-concept approaches have highlighted the therapeutic potential of AAV-gene therapy in FRDA. However, these studies have used synthetic promoters initially providing very high-yields of FXN levels and known to shutdown over time. Recent data point to the importance of maintaining frataxin levels within a range due to potential toxicity.

Methods: We generated a new AAV9-gene therapy vector encoding a DNA sequence for frataxin regulated by the hPGK1 promoter, which provides constitutive, ubiquitous, and long-term expression of frataxin in YG8R FRDA mice.

Results: Here we show that a single intrathecal administration of this vector into 2-month-old FRDA mice results in effective transduction of the recombinant frataxin gene into DRG, spinal cord, cerebellar dentate, heart, and liver, resulting in expression of recombinant frataxin protein at levels within those in WT mice, for at least 12 months with no apparent vector-induced toxicity. AAV9-FXN treated FRDA mice show preservation of motor coordination, the electrophysiological properties in sensory caudal nerve, the clasping reflex, the neuronal structural preservation of the DRG, spinal cord and dentate nucleus, along with cardiomyopathy correction, reduction of iron deposits and preservation of mitochondrial electron transport chain.

Conclusion: These data provide support for the clinical therapeutic potential of this gene therapy vector for the treatment of Friedreich's ataxia.

E. Balagué: None. D. Cota-González: None. K. AdriánCampbell: None. B. García-Lareu: None. A. Bosch: None. J. Coll-Cantí: None. M. Chillón: None. I. Sánchez: None. A. Matilla-Dueñas: None.

\section{C17.5}

HDAC inhibitor CI-994 rescues phenotype in a mouse model of Birk-Barel intellectual disability syndrome

\author{
A. Cooper ${ }^{\text {, T. Tutto }}{ }^{2 *}$, N. Hammer ${ }^{3}$, S. Jagannath ${ }^{3}$, D. \\ Lucia Fend-Guella ${ }^{l}$, J. Akhtar ${ }^{2}$, K. Radyushkin ${ }^{4}$, F. \\ Lesage $^{5}$, J. Winter ${ }^{1,6}$, S. Strand ${ }^{7}$, J. Roeper ${ }^{3}$, U. Zechner ${ }^{1,8}$, \\ S. Schweiger ${ }^{1,9}$
}

${ }^{1}$ Institute of Human Genetics, University Medical Center of the Johannes Gutenberg University, Mainz, Germany, ${ }^{2}$ Institute for Developmental Biology and Neurobiology,
Johannes Gutenberg University, Mainz, Germany, ${ }^{3}$ Institute of Neurophysiology, Goethe University Frankfurt, Germany, Frankfurt, Germany, ${ }^{4}$ University Medical Center of the Johannes Gutenberg University, Mainz, Germany, Mainz, Germany, ${ }^{5}$ LabEx ICST, Institut de Pharmacologie Moléculaire et Cellulaire, CNRS and Université de NiceSophia Antipolis, Valbonne, France, ${ }^{6}$ University Medical Center of the Johannes Gutenberg University, Mainz, Germany, ${ }^{7}$ Department of Internal Medicine I, University Medical Center of the Johannes Gutenberg University, Mainz, Germany, ${ }^{8}$ Senckenberg Center of Human Genetics, Frankfurt, Germany, ${ }^{9}$ Orphan Diseases of the Central Nervous System, University Medical Center of the Johannes Gutenberg University, Mainz, Germany

Epigenetic modulators provide a powerful tool for disease treatment through alteration of the epigenomic regulative system. In our study, we focused on the effect of epigenetic modulators on the maternally imprinted Kcnk 9 gene. Mutations in the Kcnk9 gene were shown to affect the modulation of the membrane potential and excitability of neurons, causing Birk-Barel intellectual disability syndrome (BBIDS). Using the BBIDS mouse model, we here present evidence for a small yet evident non-canonical expression of the parental $K c n k 9$ allele in $K c n k 9 \mathrm{KO}^{\text {mat }}$ animals, which was sufficient to provide an intermediate phenotype in nocturnal locomotor activity compared to $K c n k 9 \mathrm{KO}^{\text {hom }}$. We further identified the locus coeruleus (LC), a region involved in regulation of arousal and nocturnal activity in mice, as the main target region altered in $K c n k 9 \mathrm{KO}^{\text {mat }}$ animals. Following screening of numerous epigenetic modulators, we show that CI-994, a class I HDAC inhibitor, could increase paternal Kcnk9 expression up to three-fold and fully rescue the behavioral phenotype in $K c n k 9 \mathrm{KO}^{\text {mat }}$ animals. We utilized ChIP-qPCR to further demonstrate that CI-994 affects histone 3 lysine 27 acetylation (H3K27ac) at the promoter and intronic region of Kcnk9 in the LC tissue samples thereby promoting open accessible chromatin and increasing expression of Kcnk9. Interestingly, using allelespecific ChIP, we show that the effects on the inactive paternal allele were significantly higher that on the maternal allele. Taken all together, our findings provide further characterization of the role of $K c n k 9$ in BBIDS pathology as well as exciting evidence for a promising therapeutic effect of CI-994 in mouse model for BBIDS.

A. Cooper: None. T. Butto: None. N. Hammer: None. S. Jagannath: None. D. Lucia Fend-Guella: None. J. Akhtar: None. K. Radyushkin: None. F. Lesage: None. J. Winter: None. S. Strand: None. J. Roeper: None. U. Zechner: None. S. Schweiger: None. 


\section{C18 Cancer Genetics II: Clinical Cancer Research}

\section{C18.1}

Development of a rapid functional assay on peripheral blood for clinical interpretation of germline TP53 variants and detection of non-coding functional variants

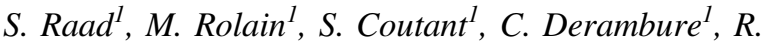

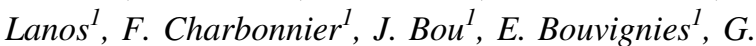 Lienard $^{\text {, S. Sasseur }}$, M. Farrel ${ }^{2}$, O. Ingster ${ }^{3}$, S. Baert- Desurmont ${ }^{l}$, E. Kasper ${ }^{l}$, G. Bougeard ${ }^{l}$, T. Frébourg ${ }^{l}$, I. Tournier $^{1 *}$}

${ }^{1}$ Inserm U1245, UNIROUEN, Normandie Univ, and Genetics Department, Rouen University Hospital, Normandy Centre for Genomics and Personalized Medicine, Rouen, France, ${ }^{2}$ Mater Private Hospital, Dublin, Ireland, ${ }^{3}$ Angers University Hospital, Department of Genetics, Angers, France

Interpretation of germline TP53 variants remains complex and is critical for an appropriate medical management of patients. We developed a functional assay directly performed on blood for the rapid interpretation, within a week, of unclassified TP53 variants. In this assay, peripheral blood mononuclear cells are cultured and activated for $48 \mathrm{~h}$ hours, exposed to doxorubicin and the p53-mediated transcriptional response is quantified using RT-MLPA and RTQMPSF, including 10 p53 targets and 2 amplicons to measure p53 mRNA levels. Functional assay and TP53 molecular screening were performed in 71 individuals, in parallel and blind conditions. In 51 wild-type TP53 individuals, the mean p53 functionality score was 12.7 . Among 8 individuals harboring likely pathogenic or pathogenic variants, the scores were reduced (mean 4.8) and p53 mRNA levels were reduced in patients harboring truncating variants allowing to identify them unambiguously. The assay applied to 11 rare unclassified variants [p.(Pro72His), p.(Gly105Asp), p.(Arg110His), p.(Phe134Leu), p. (Arg158Cys), p.(Pro191Arg), p.(Pro278Arg), p.(Arg283Cys), p.(Asp352Tyr), p.(Asn131del), c.-117G>T] led us to consider them, except the p.(Pro72His), p.(Pro191Arg) and c. $-117 \mathrm{G}>\mathrm{T}$ variants, as pathogenic. Remarkably, the assay also revealed that the c. $* 1175 \mathrm{~A}>\mathrm{C}$ polymorphic TP53 variant located within the polyadenylation signal impacts, when present on both alleles, p53 functionality with the same magnitude as a germline pathogenic TP53 variant and could act as a modifier factor in pathogenic variant carriers. This assay should therefore be a useful tool for the rapid interpretation of germline TP53 variants of unknown significance and the indirect detection of cryptic alterations within regulatory regions impacting p53 function. SR, MR: equal contribution
S. Raad: None. M. Rolain: None. S. Coutant: None. C. Derambure: None. R. Lanos: None. F. Charbonnier: None. J. Bou: None. E. Bouvignies: None. G. Lienard: None. S. Vasseur: None. M. Farrel: None. O. Ingster: None. S. BaertDesurmont: None. E. Kasper: None. G. Bougeard: None. T. Frébourg: None. I. Tournier: None.

\section{C18.2}

Are pathogenic germline variants in metastatic melanoma associated with unfavorable survival?

S. Biskup ${ }^{1 *}$, T. Amaral ${ }^{2}$, T. Sinnberg ${ }^{2}$, M. Nieser ${ }^{l}, P$. Martus $^{2}$, C. Garbe ${ }^{2}$, F. Battke ${ }^{1}$, A. Forschner ${ }^{2}$, M. Schulze ${ }^{1}$

${ }^{1}$ CeGaT, Tuebingen, Germany, ${ }^{2}$ University clinic, Tuebingen, Germany

Immunotherapy has revolutionized the treatment landscape of patients with advanced melanoma. Despite the survival improvements there are still patients that do not benefit from immunotherapy. For those patients early biomarkers that indicate potential resistance are highly relevant and other therapeutic alternatives should be considered. We comprehensively analyzed tumor and blood samples from 116 patients with advanced melanoma and scheduled for systemic therapy between January and June 2018. Descriptive and survival analysis were performed for all patients and for those receiving combined immunotherapy $(n=59)$. We focused on tumor mutational burden, genome instability, major driver and tumor suppressor genes. Interestingly, a total of 20 germline variants were identified in our cohort. Nine patients with germline variants received combined immunotherapy. The 1-year melanoma specific survival (mMSS) for the sub-group with a germline variant was 52.9 percent versus 71.9 percent for the sub-group without germline variant. For patients receiving combined immunotherapy with ipilimumab and nivolumab, the 1y-MSS was 33.3 percent and 67.2 percent respectively for patients with and without a germline variant $(p=0.019 ; 95$ percent CI: 2.5-64.1 and 54.1-80.3). The median progression-free survival was 2.0 months for patients with a germline variant and 4.0 months for those without a germline variant ( $p=0.021$; 95 percent CI: $1.5-2.5$ and 0.6-7.4). Conclusion: For the first time, we show in patients with advanced melanoma that the presence of germline variants might be associated with worse prognosis and failure in response to combined immunotherapy. Other therapeutic options should be considered early as alternative or in combination with immunotherapy.

S. Biskup: None. T. Amaral: None. T. Sinnberg: None. M. Nieser: None. P. Martus: None. C. Garbe: None. F. Battke: None. A. Forschner: None. M. Schulze: None. 


\section{C18.3}

Germline, somatic and clinical associations with response to Immune Checkpoint Inhibitors and adverse events in a large patient cohort

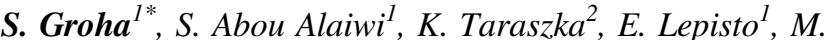 \\ Manos $^{l}$, O. Rahma ${ }^{l}$, T. Choueiri ${ }^{l}$, M. L. Freedman ${ }^{l}$, D.

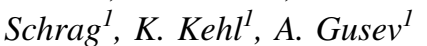

${ }^{1}$ Dana Farber Cancer Institute, Boston, MA, United States,

${ }^{2}$ UCLA, Los Angeles, CA, United States

Immune Checkpoint Inhibitors (ICIs) have led to an unprecedented improvement in durable responses in cancer therapy but only a fraction of patients derive lasting benefit, with many patients developing auto-immune disease-like adverse events (irAE), motivating research into ICI response mechanisms and biomarkers.

Using a large cohort of ICI patients at DFCI in a clinical setting ( $\mathrm{N}=1800$ pan-cancer), we probe the influence of genetic and clinical factors on overall survival (OS) and irAE incidence. We impute common germline features from panel sequenced tumors and investigate clinical, somatic and germline influences on OS and irAEs using multivariate and multistage survival analysis.

We find significant associations between OS and common germline HLA alleles $(\mathrm{q}<0.1)$, validating one association in an external cohort. We furthermore find somatic copy number alterations (CNAs) $(\mathrm{q}<0.1)$, with alterations of AKT3, RSPO2 and MYC validating in at least two independent cohorts. Moreover, we find general lab tests taken at treatment initiation are associated with OS, specific to patients on ICIs. We show that an integrated prognostic model using somatic, germline and clinical data from EHR records significantly outperforms the baseline model of demographics and tumor mutational burden (a known putative biomarker), increasing concordance from 0.63 to 0.70 . We furthermore find significant predictors of future irAEs from previous auto-immune disease diagnosis ( $\mathrm{p}<1 \mathrm{e}-$ 5 ), individual cancer driver mutations, and common HLA alleles $(\mathrm{q}<0.01)$.

Our study identifies novel mechanisms influencing OS and irAEs in patients on ICIs and shows the benefit of prognostic models utilizing clinical patient history.

NCI R01CA227237, Claudia Adams Barr Foundation

S. Groha: None. S. Abou Alaiwi: None. K. Taraszka: None. E. Lepisto: None. M. Manos: None. O. Rahma: None. T. Choueiri: B. Research Grant (principal investigator, collaborator or consultant and pending grants as well as grants already received); Modest; AstraZeneca, Alexion, Bayer, Bristol Myers-Squibb/ER Squibb and sons LLC, Cerulean, Eisai, Foundation Medicine Inc., Exelixis, Ipsen, Tracon, Genentech, Roche, Roche Products Limited, F. Hoffmann-La
Roche, GlaxoSmithKline, Lilly, Merck, Novartis, Peloton, Pfizer, Prometheus Labs, Corvus, Calithera, Analysis Group, Sanofi/Aventis, Takeda. D. Speakers Bureau/Honoraria (speakers bureau, symposia, and expert witness); Modest; AstraZeneca, Alexion, Sanofi/Aventis, Bayer, Bristol MeyerSquibb/ER Squibb and sons LLC, Cerulean, Eisai, Foundation Medicine Inc., Exelixis, Genentech, Roche, Roche Products Limited, F. Hoffmann-La Roche, GlaxoSmithKline, Merck, Novartis, Peloton, Pfizer, EMD Serono, Prometheus Labs, Corvus, Ipsen, Up-to-Date, NCCN, Analysis Group, Michael J. Hennessy (MJH) Associates, Inc (Healthcare Communications Company with several brands such as OnClive, PeerView and PER), Research to Practice, L-path, Kidney Cancer Journal, Clinical Care Options, Platform Q, Navinata Healthcare, Harborside Press, American Society of Medical Oncology, NEJM, Lancet Oncology, Heron Therapeutics, Lilly Oncology. E. Ownership Interest (stock, stock options, patent or other intellectual property); Modest; Pionyr, Tempest. F. Consultant/Advisory Board; Modest; AstraZeneca, Alexion, Sanofi/Aventis, Bayer, Bristol Myers-Squibb/ ER Squibb and sons LLC, Cerulean, Eisai, Foundation Medicine Inc., Exelixis, Genentech, Heron Therapeutics, Lilly, Roche, GlaxoSmithKline, Merck, Novartis, Peloton, Pfizer, EMD Serono, Prometheus Labs, Corvus, Ipsen, Up-todate, NCCN, Analysis Group, Pionyr, Tempest, Lilly Ventures. M. L. Freedman: None. D. Schrag: None. K. Kehl: None. A. Gusev: None.

\section{C18.4}

Rare germline variants in the tumor suppressor gene $\mathrm{CDH} 1$ are associated with familial glioma

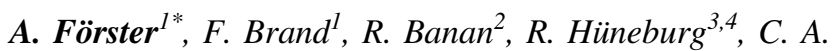

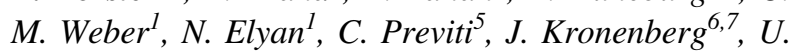

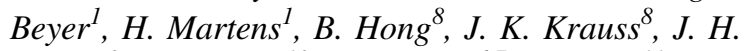
Bräsen $^{9}$, A. Zimpfer ${ }^{10}$, M. Stangel ${ }^{6,7}$, A. Samii ${ }^{11}, S$. Wolf ${ }^{5}$, S. Aretz $^{3,12}$, B. Wiese ${ }^{8,13}$, C. Hartmann ${ }^{2}$, R. G. Weber ${ }^{1}$

${ }^{I}$ Department of Human Genetics, Hannover Medical School, Hannover, Germany, ${ }^{2}$ Division of Neuropathology, Department of Pathology, Hannover Medical School, Hannover, Germany, ${ }^{3}$ National Center for Hereditary Tumor Syndromes, University Hospital Bonn, Bonn, Germany, ${ }^{4}$ Department of Internal Medicine I, University Hospital Bonn, Bonn, Germany, ${ }^{5}$ Genomics and Proteomics Core Facility, German Cancer Research Center (DKFZ), Heidelberg, Germany, ${ }^{6}$ Clinical Neuroimmunology and Neurochemistry, Department of Neurology, Hannover Medical School, Hannover, Germany, ${ }^{7}$ Center for Systems Neuroscience, University of Veterinary Medicine Hannover, Hannover, Germany, ${ }^{8}$ Department of Neurosurgery, Hannover Medical School, Hannover, Germany, ${ }^{9}$ Department of Pathology, Hannover Medical School, Hannover, Germany, 
${ }^{10}$ Institute of Pathology, University of Rostock, Rostock, Germany, ${ }^{11}$ Department of Neurosurgery, International Neuroscience Institute, Hannover, Germany, ${ }^{12}$ Institute of Human Genetics, University Hospital Bonn, Bonn, Germany, ${ }^{13}$ Department of Neurology, Henriettenstift, Diakovere Krankenhaus gGmbH, Hannover, Germany

The genetic basis of familial gliomas, representing about $5 \%$ of all gliomas, is poorly understood. In search of rare germline alterations predisposing to glioma, we applied whole-genome, whole-exome and targeted sequencing to germline-DNA of 21 glioma patients from 15 families with at least two glioma cases each. Thereby, we identified two families with rare variants in the tumor suppressor gene $\mathrm{CDH1}$ encoding E-cadherin that co-segregate with the tumor phenotype, a significant overrepresentation of rare $\mathrm{CDH1}$ variants in glioma families versus controls. In a separate cohort of 45 individuals from 25 gastric cancer families carrying $\mathrm{CDH} 1$ germline mutations, brain tumors were observed in two cases, a significantly higher prevalence than in the general population. As most rare $\mathrm{CDHI}$ variant carriers in the two glioma families were affected by oligodendroglioma, we performed mutational analysis in a separate cohort of 101 oligodendroglioma-DNAs identifying rare $\mathrm{CDHl}$ alterations in about $6 \%$ of tumors. $\mathrm{CDH}$ expression was detected in oligodendroglial precursor cells and differentiated oligodendrocytes isolated from rat brain. Functional studies of $\mathrm{CDHl}$ germline variants using CRISPR/Cas9-mediated knock-in or stably transfected cell models demonstrated their pathogenicity with respect to cell surface expression, cell migration and aggregation compared to control cells. One $C D H I$ variant showed reduced binding to beta-catenin and increased nuclear levels of active beta-catenin that were diminished by treatment with the MAPK interacting serine/threonine kinase 1 inhibitor CGP57380. Our data provide evidence for a role of rare $\mathrm{CDHl}$ variants in the risk and tumorigenesis of gliomas, particularly of oligodendrogliomas (supported by Wilhelm Sander-Stiftung grant 2018.097.1).

A. Förster: None. F. Brand: None. R. Banan: None. R. Hüneburg: None. C. A. M. Weber: None. N. Elyan: None. C. Previti: None. J. Kronenberg: None. U. Beyer: None. H. Martens: None. B. Hong: None. J. K. Krauss: None. J. H. Bräsen: None. A. Zimpfer: None. M. Stangel: None. A. Samii: None. S. Wolf: None. S. Aretz: None. B. Wiese: None. C. Hartmann: None. R. G. Weber: None.

\section{C18.5}

Prostate cancer risk and prognosis for carriers of germline pathogenic variants in disease implicated genes

\author{
N. Camacho Ordonez , L. Dong, A. Matakidou
}

AstraZeneca, Melbourn, Royston, Hertfordshire, United Kingdom

Germline mutations in DNA Damage Response (DDR) genes such as BRCA2 and ATM have been associated with prostate cancer risk and agressiveness. These associations are largely based on studies that ascertain for cancer diagnosis and family history and provide risk estimates with limited population-level accuracy. Here we evaluate the clinical significance of germline pathogenic variants in 20 DDR genes and four known susceptibility coding variants in POUF51B, HOXB13 and KLK3 using whole exome sequences from (1) the UK Biobank (UKBB), a populationbased cohort (200,000 participants) and (2) patients recruited in AZ clinical trials. Whole exomes from 4,975 prostate cancer patients $(3,954$ UKBB and 1,021 AZ) and 59,438 cancer-free males were analysed. Known and novel pathogenic variants were identified and population risk of disease, metastasis and overall survival (UKBB only) were estimated (multiplicity corrected $\mathrm{P}$ value $<0.005$ ). ATM (1.15\%), BRCA2 (1.11\%) and HOXB13 G48E (1.29\%) were the largest contributors to prostate cancer risk, each conferring an increase of $\sim 4$-fold; followed by $C H E K 2$ with a moderate contribution $(0.4 \%)$. BRCA2 carriers were more likely to metastasise (OR 3.32, 95\% CI 1.74-6.45, $\mathrm{p}=9.94 \mathrm{E}-05)$ and had poorer overall survival (Log-Rank $\mathrm{p}=1.33 \mathrm{E}-08$ ), with $85 \%$ dying from prostate cancer compared to $53 \%$ of non-BRCA2 carriers. This is the largest study to date providing population-based estimates of prostate cancer risk, metastasis an survival. Analyses will be updated with data from the full 500,000 UKBB participants (11,000 prostate cancers).

N. Camacho Ordonez: A. Employment (full or parttime); Significant; AstraZeneca. L. Dong: A. Employment (full or part-time); Significant; AstraZeneca. A. Matakidou: A. Employment (full or part-time); Significant; AstraZeneca.

\section{C18.6}

Clinical and molecular characterization of 1253 carriers of a deleterious CHEK2 mutation from the German Consortium for Hereditary Breast and Ovarian Cancer (GC-HBOC)

W. Habhab ${ }^{1 *}$, U. Faust ${ }^{l}$, G. Günther ${ }^{1}$, U. Siebers-Renelt ${ }^{2}$, M. Kiechle ${ }^{3}$, C. Ott ${ }^{4}$, N. Dikow ${ }^{5}, K$. Kast $^{6}$, A. Vesper ${ }^{7}$, C. Solbach $^{8}$, N. Harbeck ${ }^{9}$, M. Stiller ${ }^{10}$, A. Gehrig ${ }^{11}, C$. Thomssen $^{12}$, H. Wallaschek ${ }^{13}$, N. Arnold ${ }^{14}$, I. Holzhauser ${ }^{15}$, S. Kaulfu $\beta^{16}, A$. Volk $^{17}$, W. Janni ${ }^{18}, C$. Engel ${ }^{19}, R$. Schmutzler $^{20}$, O. Rieß ${ }^{l}$, C. Schroeder ${ }^{l}$, K. Bosse ${ }^{l}$

${ }^{1}$ Institute of Medical Genetics and Applied Genomics, University of Tuebingen, Tuebingen, Germany, ${ }^{2}$ Institute 
of Human Genetics, University of Muenster, Muenster, Germany, ${ }^{3}$ Department of Gynecology, University Hospital, Technical University of Munich, Munich, Germany, ${ }^{4}$ Institute of Medical Genetics and Human Genetics, Charité University medicine of Berlin, Berlin, Germany, ${ }^{5}$ Institute of Human Genetics, University Hospital Heidelberg, Heidelberg, Germany, ${ }^{6}$ Department of Gynecology and Obstetrics, Medical Faculty and University Hospital Carl Gustav Carus, Technische University Dresden, Dresden, Germany, ${ }^{7}$ Department of Gynecology and Obstetrics, Medical Faculty and University Hospital Duesseldorf, HeinrichHeine University Duesseldorf, Duesseldorf, Germany, ${ }^{8}$ Department of Obstetrics \& Gynecology, University Hospital, Frakfurt, Germany, ${ }^{9}$ Department of Obstetrics \& Gynecology, University of Munich (LMU), Munich, Germany, ${ }^{10}$ Institute of Human Genetics, University of Leipzig Hospitals and Clinics, Leipzig, Germany, ${ }^{11}$ Institute of Human Genetics, University of Wuerzburg, Wuerzburg, Germany, ${ }^{12}$ Department of Gynecology and Obstetrics, Medical Faculty and University Hospital Halle, Halle, Germany, ${ }^{13}$ Institute of Human Genetics, University Hospital of Hannover, Hannover, Germany, ${ }^{14}$ Department of Gynecology and Obstetrics, University Hospital Schleswig-Holstein, Campus Kiel, Christian Albrechts University Kiel Institute of Clinical Molecular Biology, University Hospital of Schleswig-Holstein, Campus Kiel, ChristianAlbrechts Universi, Kiel, Germany, ${ }^{15}$ Institute of Human Genetics, University of Regensburg, Regensburg, Germany, ${ }^{16}$ Institute of Human Genetics, University Medical Center Goettingen, Goettingen, Germany, ${ }^{17}$ Institute of Human Genetics, University Medical Center Hamburg-Eppendorf, Hamburg, Germany, ${ }^{18}$ Department of Obstetrics and Gynecology, University Clinic Ulm, Ulm, Germany, ${ }^{19}$ Institute of Medical Informatics, Statistics and Epidemiology, University of Leipzig, Leipzig, Germany, ${ }^{20}$ Center of Hereditary Breast and Ovarian Cancer, University of Cologne, Cologne, Germany

Introduction: Published relative risk estimates for breast cancer for $C H E K 2$ mutation carriers vary between 1.5 and 3. The risk for contralateral breast cancer is elevated. An association with ovarian cancer is still under debate. Here we present the clinical and molecular characteristics of 1253 female and male CHEK2 mutation carriers recruited by the German Consortium for Hereditary Breast and Ovarian Cancer (GC-HBOC).

Materials and Methods: Deleterious mutations in BRCA1 and BRCA2 had been excluded in the study group. The information was gained from the central registry database of the Consortium.

Results: A total of 1253 patients were tested positive for a pathogenic germline CHEK2 variant (1176 females, 77 males). More than $60 \%$ of the female carriers had developed breast- and/or ovarian cancer. Approximately $13 \%$ of the females with breast cancer reported a contralateral cancer. Ovarian cancer was diagnosed in 35 carriers $(2.98 \% ; 13$ of them $<50 \mathrm{yrs}$ ). About $6.5 \%$ of the males developed breast cancer. Sixteen mutation carriers developed skin and 14 thyroid cancer, respectively. The most common observed variant was c.1100delC followed by Del Ex9-10.

Conclusion: $C H E K 2$-mutations are a substantial cause of hereditary female breast cancer in the German population. Female carriers face a relevant risk of developing contralateral cancer. CHEK2 mutation carriers present with a broad spectrum of additional cancers. Besides ovarian cancer, thyroid and skin cancer are the most frequent entities. A causal association with an increased risk of ovarian cancer and male breast cancer remains to be proven in larger study cohorts and matched to healthy controls.

W. Habhab: None. U. Faust: None. G. Günther: None. U. Siebers-Renelt: None. M. Kiechle: None. C. Ott: None. N. Dikow: None. K. Kast: None. A. Vesper: None. C. Solbach: None. N. Harbeck: None. M. Stiller: None. A. Gehrig: None. C. Thomssen: None. H. Wallaschek: None. N. Arnold: None. I. Holzhauser: None. S. Kaulfuß: None. A. Volk: None. W. Janni: None. C. Engel: None. R. Schmutzler: None. O. Rieß: None. C. Schroeder: None. K. Bosse: None.

\section{C19 Multiple Malformation Syndromes II}

\section{C19.1}

One test for all: whole genome sequencing in 62 trios with congenital limb malformation identifies structural variants and new disease genes

\section{J. Elsner ${ }^{l}$, M. A. Mensah ${ }^{1}$, M. Holtgrewe ${ }^{2}$, W. Hülsemann ${ }^{3}$, S. Mundlos ${ }^{4,1}$, M. Spielmann ${ }^{4,1^{*}}$ \\ ${ }^{1}$ Institute of Medical Genetics and Human Genetics, Charité - Universitätsmedizin Berlin, Berlin, Germany, ${ }^{2}$ Core Unit Bioinformatics, Berlin Institute of Health, Berlin, Germany, ${ }^{3}$ Handchirurgie, Katholisches Kinderk- rankenhaus Wilhelmstift, Hamburg, Germany, ${ }^{4}$ Max Planck Institute for Molecular Genetics, Berlin, Germany}

Introduction: The extensive clinical and genetic heterogeneity of congenital limb malformation requires comprehensive analysis of genome-wide genetic variation. Whole genome sequencing (WGS) has the potential to identify both coding and non-coding as well as structural variants in a single test. Thus, WGS may increase the diagnostic rate in cases of congenital limb malformation.

Methods: We collected a cohort of 62 trios featuring congenital limb malformations that were mutation negative after standard clinical genetic testing. The cohort was 
subjected to whole genome sequencing (Ilumina, 30x coverage, paired-end, 150bp). Data were analyzed for both coding and non-coding single nucleotide variants and structural variants using the SODAR and VarFish pipelines.

Results: Overall we identified coding pathogenic single nucleotide variants in 9 cases (14\%). We found mutations in 7 known disease genes including one repeat expansion in HOXD13. In two unrelated cases of ectrodactyly we identified likely pathogenic variants in UBA2 establishing it as a novel disease gene. In addition we found two pathogenic structural variants $(3 \%)$ : one inversion segregating with the phenotype and one de novo deletion. Furthermore, we identified variants in 3 novel high confidence candidate genes. We also describe and perform a framework to identify potential non-coding pathogenic variants.

Conclusions: Whole genome sequencing is a powerful strategy to identify all types of genomic variants associated with congenital limb malformation. It also detects repeat expansions and copy number neutral inversions that are missed by most other approaches.

J. Elsner: None. M. A. Mensah: None. M. Holtgrewe: None. W. Hülsemann: None. S. Mundlos: None. M. Spielmann: None.

\section{C19.2}

Biallelic mutations in TOGARAM1 cause a novel primary ciliopathy

V. Morbidoni ${ }^{1,2}$, E. Agolini ${ }^{3}$, K. C. Slept ${ }^{4}$, L. Pannone ${ }^{5}$, D.

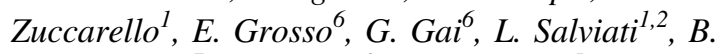

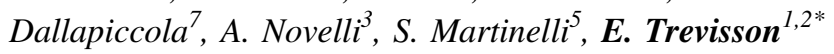

${ }^{1}$ Clinical Genetics Unit, Dept of Women's and Children's Health, University of Padova, Padova, Italy, ${ }^{2}$ Istituto di Ricerca Pediatrica IRP, Padova, Italy, ${ }^{3}$ Laboratory of Medical Genetics, IRCCS-Bambino Gesù Children's Hospital, Roma, Italy, ${ }^{4}$ Department of Biology, University of North Carolina, Chapel Hill, NC, United States, ${ }^{5}$ Department of Oncology and Molecular Medicine, Istituto Superiore di Sanità, Roma, Italy, ${ }^{6}$ Medical Genetics Unit, Città della Salute e della Scienza University Hospital, Torino, Italy, ${ }^{7}$ Scientific Directorate, Bambino Gesù Children's Hospital and Research Institute, Roma, Italy

Background: Dysfunction in non-motile cilia is associated with a broad spectrum of developmental disorders characterized by clinical heterogeneity. Despite over one hundred genes have been associated with primary ciliopathies, with wide phenotypic overlap, some patients still lack a molecular diagnosis. The aim of this work was to investigate and functionally characterize the molecular cause underlying a Meckel-Gruber-like phenotype in two sibling foetuses.

Methods: we used a trio-based whole exome sequencing (WES) strategy to identify candidate variants in TOGARAM1. In silico, in vitro and in vivo (C. elegans) studies were carried out to explore the impact of mutations on protein structure and function, and relevant biological processes.

Results: TOGARAM1 encodes a member of the Crescerin 1 family of proteins regulating microtubule dynamics. Its orthologue in C. elegans, che-12, is expressed in the cilium of a subset of sensory neurons, where it is required for chemosensation. Nematode lines harbouring the missense variant in TOGARAM1 were generated by CRISPR/ Cas9 technology. Although chemotaxis ability on a $\mathrm{NaCl}$ gradient and lipophilic dye-uptake were not impaired, che12 knock-in mutants displayed a shorter cilium in sensory neurons, and even shorter cilia in hemizygous worms, which recapitulate the genotype observed in the affected foetuses. Finally, in vitro analysis of microtubule polymerization in the presence of the wild-type or mutant TOG2 domain revealed a faster polymerization associated with the mutant protein, indicating aberrant tubulin binding.

Conclusions: Our data suggest a causative role of TOGARAM1 variants in the pathogenesis of this novel disorder, connecting this gene with primary ciliopathy.

V. Morbidoni: None. E. Agolini: None. K. C. Slept: None. L. Pannone: None. D. Zuccarello: None. E. Grosso: None. G. Gai: None. L. Salviati: None. B. Dallapiccola: None. A. Novelli: None. S. Martinelli: None. E. Trevisson: None.

\section{C19.3}

Biallelic loss-of-function variations in SMO, encoding the key transducer of the Sonic Hedgehog pathway, cause a broad phenotypic spectrum of hedgehogopathies

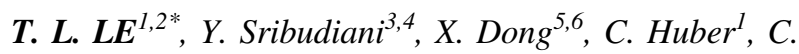
Kois $^{7}$, G. Baujat ${ }^{1,8}$, C. T. Gordon ${ }^{1}$, V. Mayne ${ }^{5}$, L. Galmiche $^{8}$, V. Serre ${ }^{9,2}$, N. Goudin ${ }^{2}$, M. Zarhrate ${ }^{1}$, C. Bole-

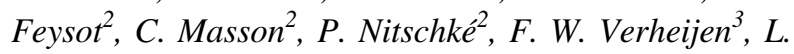
Pais $^{10}$, A. Pelet ${ }^{1}$, S. Sadedin ${ }^{5}$, J. A. Pugh ${ }^{7}$, N. Shur ${ }^{11}$, S. M. White $^{5}$, S. E. Chehadeh ${ }^{12}$, J. Christodoulou ${ }^{5,6}$, V. CormierDaire $^{1,8}$, R. M. W. Hofstra ${ }^{3}$, S. Lyonnet ${ }^{1,2,8}$, T. Y. Tan ${ }^{5,6}$, T. Attié-Bitach $^{1,2,8}$, W. S. Kerstjens-Frederikse ${ }^{13}$, J. Amiel ${ }^{1,2,8}$, S. Thomas ${ }^{l}$

${ }^{1}$ Imagine Institute - INSERM U1163, Paris, France, ${ }^{2}$ Université de Paris, Paris, France, ${ }^{3}$ Erasmus Medical Center, Rotterdam, Netherlands, ${ }^{4}$ Universitas Padjadjaran, Bandung, Indonesia, ${ }^{5}$ Royal Children's Hospital, Melbourne, Australia, ${ }^{6}$ University of Melbourne, Melbourne, Australia, ${ }^{7}$ Albany Medical Center, Albany, NY, United States, ${ }^{8}$ Hôpital Necker-Enfants Malades, Paris, France, ${ }^{9}$ Institut 
Jacques Monod - CNRS U7592, Paris, France, ${ }^{10}$ Broad Institute, Cambridge, MA, United States, ${ }^{11}$ Children's National, Washington, WA, United States, ${ }^{12}$ Hôpital de Hautepierre, Strasbourg, France, ${ }^{13}$ University Medical Centre Groningen, Groningen, Netherlands

The evolutionarily conserved Hedgehog (Hh) pathway is essential for organogenesis and plays critical roles in postnatal tissue maintenance and renewal. A unique feature of vertebrate Hh pathway is that signal transduction requires the primary cilium (PC) where major pathway components are dynamically enriched. These factors include Smoothened (SMO) and Patched, that constitute the core reception system for Sonic Hedgehog (SHH) as well as GLI transcription factors, the key mediators of the pathway. Here, we report biallelic loss-of-function variations in $S M O$ in seven cases from five independent families causing a wide phenotypic spectrum of hedgehogopathies affecting development of the brain (hypothalamic hamartoma, microcephaly), heart (atrioventricular septal defect), skeleton (postaxial polydactyly, narrow chest, shortening of long bones) and the enteric nervous system (aganglionosis). Patient-derived cells showed normal ciliogenesis but severely altered $\mathrm{Hh}$ signal transduction due to either altered PC trafficking or abnormal activation of the pathway downstream of SMO. In addition, Hh-independent GLI2 accumulation at the PC tip in patient cells suggest a potential novel function of SMO in regulating basal ciliary trafficking of GLI2 when the pathway is off. Thus, loss of SMO function in humans results in abnormal PC dynamics of key components of the Hh signaling pathway and leads to a large continuum of malformations in humans.

T. L. Le: None. Y. Sribudiani: None. X. Dong: None. C. Huber: None. C. Kois: None. G. Baujat: None. C. T. Gordon: None. V. Mayne: None. L. Galmiche: None. V. Serre: None. N. Goudin: None. M. Zarhrate: None. C. Bole-Feysot: None. C. Masson: None. P. Nitschké: None. F. W. Verheijen: None. L. Pais: None. A. Pelet: None. S. Sadedin: None. J. A. Pugh: None. N. Shur: None. S. M. White: None. S. E. Chehadeh: None. J. Christodoulou: None. V. Cormier-Daire: None. R. M. W. Hofstra: None. S. Lyonnet: None. T. Y. Tan: None. T. Attié-Bitach: None. W. S. Kerstjens-Frederikse: None. J. Amiel: None. S. Thomas: None.

\section{C19.4}

Bi-allelic Variants in RALGAPA1 Cause Profound Neurodevelopmental Disability, Muscular Hypotonia, Infantile Spasms, and Feeding Abnormalities

M. Wagner $^{1,2 *}$, Y. Skorobogatko ${ }^{3}$, B. Pode-Shakked ${ }^{4}$, C. M. Powell ${ }^{5}$, B. Alhaddad ${ }^{1}$, A. Seibt ${ }^{6}$, O. Barel ${ }^{7}$, G. Heimer ${ }^{7}$, C. Hoffmann $^{4}$, L. A. Demmer ${ }^{8}$, Y. Perilla-Young ${ }^{5}$, M. Remke ${ }^{9}$, D. Wieczorek ${ }^{10}$, T. Navaratnarajah ${ }^{6}$, P. Lichtner ${ }^{11}, D$.
Klee $^{12}$, H. E. Shamseldin ${ }^{13}$, F. A. Mutairi ${ }^{14}$, E. Mayatepek ${ }^{6}$, T. M. Strom ${ }^{1}$, T. Meitinger ${ }^{1}$, F. S. Alkuraya ${ }^{13}$, Y. Anikster ${ }^{7}$, A. R. Saltiel ${ }^{3}, F$. Distelmaier ${ }^{6}$

${ }^{1}$ Institute of Human Genetics, Munich, Germany, ${ }^{2}$ Institute for Neurogenomics, Helmholtz Zentrum München, Neu, Germany, ${ }^{3}$ San Diego School of Medicine, La Jolla, CA, United States, ${ }^{4}$ Sheba Medical Center, Tel Aviv, Israel, ${ }^{5}$ Department of Pediatrics, Chapel Hill, NC, United States, ${ }^{6}$ University Children's Hospital, Düsseldorf, Germany, ${ }^{7}$ Sheba Medical Center, Tel-Hashomer, Israel, ${ }^{8}$ Atrium Health Levine Children's Hospital, Charlotte, NC, United States, ${ }^{9}$ Department of Pediatric Oncology, Düsseldorf, Germany, ${ }^{10}$ Institute of Human Genetics, Düsseldorf, Germany, ${ }^{11}$ Institute of Human Genetics, Neuherberg, Germany, ${ }^{12}$ Department of Diagnostic and Interventional Radiology, Düsseldorf, Germany, ${ }^{13}$ King Faisal Specialist Hospital and Research Center, Riyadh, Saudi Arabia, ${ }^{14}$ King Abdulaziz Medical City, Riyadh, Saudi Arabia

Introduction: Ral (Ras-like) GTPases play an important role in the control of cell migration and have been implicated in Ras-mediated tumorigenicity. Recently, variants in RALA were described as a cause of intellectual disability and developmental delay, indicating the relevance of this pathway to neuropediatric diseases.

Materials and Methods: Exome sequencing was performed in four affected individuals. Immunoblotting, qRTPCR, immunofluorescence, flow cytometry and a drug screen pipeline using cell viability were used to functionally characterize patient derived fibroblasts.

Results: Biallelic variants in $R A L G A P A l$ were identified in four unrelated individuals with profound neurodevelopmental disability, muscular hypotonia, feeding abnormalities, recurrent fever episodes, and infantile spasms. RalGAPA1 was absent in the fibroblasts derived from two affected individuals and levels of RalGAPB, a scaffolding subunit of the RalGAP complex, were reduced. RalA activity was increased in these cell lines, implying that RalGAPA1 deficiency causes a dysfunctional RalGAP complex and a constitutive activation of RalA. Additionally, RalGAPA1 deficiency increased cell-surface levels of lipid raft components in detached fibroblasts, indicating that anchorage-dependence of cell growth signaling is disturbed. The drug screen assay showed altered survival rates of cells upon treatment with pathway specific compounds.

Conclusions: Our findings indicate that the dysregulation of the RalA pathway has an important impact on neuronal function and brain development. In light of the partially overlapping phenotype between RALA and RALGAPA1associated diseases, it appears likely that dysregulation of the RalA signaling pathway leads to a distinct group of 
genetic syndromes that we suggest could be named RALopathies.

M. Wagner: None. Y. Skorobogatko: None. B. PodeShakked: None. C. M. Powell: None. B. Alhaddad: None. A. Seibt: None. O. Barel: None. G. Heimer: None. C. Hoffmann: None. L. A. Demmer: None. Y. Perilla-Young: None. M. Remke: None. D. Wieczorek: None. T. Navaratnarajah: None. P. Lichtner: None. D. Klee: None. H. E. Shamseldin: None. F. A. Mutairi: None. E. Mayatepek: None. T. M. Strom: None. T. Meitinger: None. F. S. Alkuraya: None. Y. Anikster: None. A. R. Saltiel: None. F. Distelmaier: None.

\section{C19.5}

Functional genomics and extended analyses of unsolved exome-negative cases through the Undiagnosed Diseases Project in Victoria (UDP-Vic)

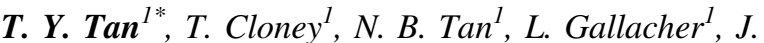
Elliott ${ }^{1}$, G. Helman ${ }^{2}$, C. Simons ${ }^{2}$, S. Sadedin ${ }^{1}$, L. Pais ${ }^{3}$, A. O'Donnell-Luria ${ }^{3}$, J. Christodoulou ${ }^{2}$, S. M. White ${ }^{l}$

${ }^{1}$ Victorian Clinical Genetics Services, Parkville, Melbourne, Australia, ${ }^{2}$ Murdoch Children's Research Institute, Parkville, Melbourne, Australia, ${ }^{3}$ Broad Center for Mendelian Genomics, Cambridge, MA, United States

Clinical exome sequencing (cES) achieves a diagnostic yield of $40-50 \%$ in individuals with a suspected monogenic disorder. Optimal strategies for solving cES-negative cases are unclear. Aim: Develop a research program to identify diagnoses in unsolved cES-negative individuals. Methods: We established the Undiagnosed Diseases Project in Victoria (UDP-Vic) in collaboration with the Broad Center for Mendelian Genomics to undertake family-based sequencing and multifaceted approaches including genomic re-analysis, copy number variant identification, RNA-sequencing and functional studies. Results: We recruited $288 \mathrm{cES}$-negative families to UDP-Vic for family-based exome (ES; 263) or genome sequencing (GS; 25). Probands are paediatric with syndromic neurodevelopmental phenotypes. Analysis is complete in 133 families, of which 56 (42.1\%) are considered solved (comprising known, newly emerging, and strong novel candidate genes). Family-based ES/GS was the most useful strategy in identifying candidate variants, and adjunctive analyses such as RNA-sequencing (4/18 cases analysed; $22.2 \%$ ), high-resolution microarray (2/32 cases analysed; $6.3 \%)$; re-analysis of original data (2/12 cases analysed; $16.7 \%$ ) provided additional diagnoses. GS identified only one coding variant that was missed in the original cES. We identified strong candidates in 20 novel disease genes, of which data-sharing and functional analyses led to publication of 6 (MSL3, THOC2, FIG4, HNRNPU, SYT1,
ZNF462). Cell-based and animal model functional analyses are ongoing for other novel candidates. Conclusion: UDPVic has been highly effective in extending the diagnostic yield beyond that achieved by cES. Our multifaceted strategy has enabled us to query clinically-driven hypotheses and identify cryptic diagnoses. We plan to incorporate additional '-omics' approaches to our pipeline.

T. Y. Tan: None. T. Cloney: None. N. B. Tan: None. L. Gallacher: None. J. Elliott: None. G. Helman: None. C. Simons: None. S. Sadedin: None. L. Pais: None. A. O'Donnell-Luria: None. J. Christodoulou: None. S. M. White: None.

\section{C19.6}

Identification of DNA methylation episignatures for PRC2-related overgrowth syndromes

M. A. Levy ${ }^{1,2 *}$, E. Aref-Eshghi ${ }^{1}$, T. B. Balci ${ }^{1,2,3}$, J. Kerkhof ${ }^{1}$, G. Merla ${ }^{4}$, C. Schwartz ${ }^{5}$ B. Sadikovic ${ }^{1,2}$

${ }^{1}$ London Health Sciences Centre, London, ON, Canada, ${ }^{2}$ Western University, London, ON, Canada, ${ }^{3}$ Lawson Health Research Institute, London, ON, Canada, ${ }^{4}$ IRCCSCasa Sollievo della Sofferenza, San Giovanni Rotondo, Italy, ${ }^{5}$ Greenwood Genetic Center, Greenwood, CT, United States

Introduction: An expanding number of genetic syndromes have been shown to have unique genomic DNA methylation patterns (episignatures). EZH2 and EED are core components of the polycomb repressive complex 2 (PRC2) chromatin remodeling complex which catalyzes methylation of lysine 27 on histone H3 (H3K27). Mutations in these genes lead to the overgrowth/intellectual disability syndromes Weaver syndrome (EZH2) or Cohen-Gibson syndrome (EED).

Materials and Methods: We used DNA methylation microarrays which interrogate 450,000-850,000 individual CpGs, along with machine learning statistical techniques, to identify EZH2- and EED-specific DNA methylation signatures present in peripheral blood from affected individuals.

Results: Using these signatures we differentiated between individuals with EZH2 or EED mutations, controls, and those with a wide range of other developmental disability/ intellectual disability (DD/ID) syndromes. In addition, we were able to distinguish between pathogenic and benign mutations in either gene, demonstrating the usefulness of these DNA methylation signatures for classifying variants of uncertain significance. Weaver and Cohen-Gibson syndromes exhibit overlapping clinical characteristics and their methylation signatures reflect this. 
Conclusions: These results contribute to the growing catalogue of DNA methylation episignatures useful for the clinical diagnosis of DD/ID and related syndromes.

M. A. Levy: None. E. Aref-Eshghi: None. T. B. Balci: None. J. Kerkhof: None. G. Merla: None. C. Schwartz: None. B. Sadikovic: None.

\section{C20 Neurogenetics}

\section{C20.1}

An ancestral 10bp repeat expansion in gene encoding component of ECM causes a novel autosomal-recessive peripheral neuropathy

A. T. Pagnamenta ${ }^{1 *}, Y . Z^{2} u^{2}$, S. Donkervoort ${ }^{2}$, S. B. Neuhaus $^{2}$, R. Maroofian ${ }^{3}$, N. Dominik ${ }^{3}$, H. Y. Yip ${ }^{3}$, A. H. Nemeth ${ }^{4,5}$, M. O'Driscoll ${ }^{6}, F$. Norwood ${ }^{7}, J_{\text {J Rankin }}^{8}, T$. Lavin $^{9}$, C. Marini-Bettolo ${ }^{10,11}$, H. Jungbluth ${ }^{12,13,14}$, L. Medne ${ }^{15}$, S. Y. Yum ${ }^{16}$, A. R. Foley ${ }^{2}$, A. Need ${ }^{17}$, M. Sedghi ${ }^{18}$, B. Ansari ${ }^{19}$, K. Basiri $^{19}$, A. R. Moslemi ${ }^{20}$, H. Tajsharghi ${ }^{21}$, C. C. Muraresku ${ }^{22}$, Z. Zolkipli-Cunningham ${ }^{22}$, S. Lassche ${ }^{23}$, C. Marcelis ${ }^{23}$, Genomics England Research Consortium, J. C. Taylor ${ }^{1}$, C. G. Bönnemann ${ }^{2}$, H. Houlden ${ }^{3}$

${ }^{I}$ Wellcome Centre for Human Genetics, University of Oxford, Oxford, United Kingdom, ${ }^{2}$ Neuromuscular and Neurogenetic Disorders of Childhood Section, NINDS, National Institutes of Health, Bethesda, MD, United States, ${ }^{3}$ Department of Neuromuscular Disorders, UCL Queen Square Institute of Neurology, London, United Kingdom, ${ }^{4}$ Nuffield Department of Clinical Neurosciences, University of Oxford, Oxford, United Kingdom, ${ }^{5}$ Oxford Centre for Genomic Medicine, Oxford University Hospitals NHS Trust, Oxford, United Kingdom, ${ }^{6}$ Birmingham Women's NHS Foundation Trust, Birmingham, United Kingdom, ${ }^{7}$ Department of Neurology, King's College Hospital, London, United Kingdom, ${ }^{8}$ Peninsula Clinical Genetics Service, Royal Devon and Exeter NHS Trust, Exeter, United Kingdom, ${ }^{9}$ Department of Neurology, Salford Royal NHS Foundation Trust, Manchester, United Kingdom, ${ }^{10}$ The John Walton Muscular Dystrophy Research Centre, Institute of Genetic Medicine, Newcastle University, Newcastle, United Kingdom, ${ }^{11}$ Newcastle upon Tyne Hospitals NHS Foundation Trust, Newcastle, United Kingdom, ${ }^{12}$ Department of Paediatric Neurology - Neuromuscular Service, Evelina Children's Hospital, Guy's \& St Thomas' NHS Foundation Trust, London, United Kingdom, ${ }^{13}$ Randall Division of Cell and Molecular Biophysics Muscle Signalling Section, King's College London, London, United Kingdom, ${ }^{14}$ Department of Basic and Clinical Neuroscience, Institute of Psychiatry, Psychology and Neuroscience, King's College London, London, United Kingdom, ${ }^{15}$ Divisions of Neurology and Human Genetics of Genomic Medicine, Children's Hospital of Philadelphia, Philadelphia, PA, United States, ${ }^{16}$ Division of Pediatric Neurology, The Children's Hospital of Philadelphia, Perelman School of Medicine at the University of Pennsylvania, Philadelphia, PA, United States, ${ }^{17}$ William Harvey Research Institute, Queen Mary University of London, London, United Kingdom, ${ }^{18}$ Medical Genetics Laboratory, Alzahra University Hospital, Isfahan University of Medical Sciences, Isfahan, Iran, Islamic Republic of, ${ }^{19}$ Department of neurology, Faculty of Medicine, Isfahan University of Medical Sciences, Isfahan, Iran, Islamic Republic of, ${ }^{20}$ Department of Pathology, University of Gothenburg, Sahlgrenska University Hospital, Gothenburg, Sweden, ${ }^{21}$ School of Health Science, Division Biomedicine and Translational Medicine, University of Skovde, Skovde, Sweden, ${ }^{22}$ Mitochondrial Medicine Frontier Program, Division of Human Genetics, Children's Hospital of Philadelphia, Philadelphia, PA, United States, ${ }^{23}$ Radboud University Medical Centre, Nijmegen, Netherlands

The extracellular matrix (ECM) comprises a network of macromolecules such as collagens, proteoglycans and glycoproteins. VWAI encodes a component of the ECM that interacts with perlecan/collagen VI, is essential in stabilizing ECM structures and demonstrates high expression levels in tibial nerve. Vwa1-deficient mice manifest with abnormal peripheral nerve structure and function, however VWAl variants have not yet been associated with disease in humans. Interrogation of genome sequences of 74,180 individuals from the $100 \mathrm{~K}$ Genomes Project yielded 10 unrelated patients with rare biallelic variants in VWAI (NM_022834.5). A single disease-associated allele (c.62_71dup10; p.G25Rfs*74) was homozygous in 7 individuals and compound-heterozygous with other likelydeleterious variants in another 3. Given an AF of $\sim 1 / 1000$, observation of 7 homozygotes constitutes a substantial enrichment from expectation, consistent with the variant playing a causative role in the patients' disease. The wide age-range of patients (6-70 years) helps us delineate the clinical phenotype over time. Initial assessment suggests a consistent clinical presentation with common features including muscle weakness, motor delay, pes cavus and SMA-like electrophysiology. Additional families with biallelic variants in VWA1 have been identified through collaborative efforts and partial nonsense mediated decay of mRNA was observed in fibroblasts. Ongoing work includes targeted-screening of a large cohort of unsolved CMT patients. We hypothesise that this disease gene has previously evaded detection using exome sequencing due to high GC content, consequential low coverage and computational difficulties associated with robustly detecting repeat-expansions. It is anticipated that review of previously 
unsolved exomes using lower QC filters may generate further diagnoses.

A. T. Pagnamenta: None. Y. Zou: None. S. Donkervoort: None. S. B. Neuhaus: None. R. Maroofian: None. N. Dominik: None. H. Y. Yip: None. A. H. Nemeth: None. M. O'Driscoll: None. F. Norwood: None. J. Rankin: None. T. Lavin: None. C. Marini-Bettolo: None. H. Jungbluth: None. L. Medne: None. S. Y. Yum: None. A. R. Foley: None. A. Need: None. M. Sedghi: None. B. Ansari: None. K. Basiri: None. A. R. Moslemi: None. H. Tajsharghi: None. C. C. Muraresku: None. Z. Zolkipli-Cunningham: None. S. Lassche: None. C. Marcelis: None. J. C. Taylor: None. C. G. Bönnemann: None. H. Houlden: None.

\section{C20.2}

Mutations in the Golgi protein GBF1 as a novel cause of distal hereditary motor neuropathy

N. Mendoza Ferreira ${ }^{1}$, M. Karakaya ${ }^{l}, N$. Cengiz ${ }^{l}, D$. Beijer $^{2}$, N. Fuhrmann ${ }^{l}$, I. Hölker ${ }^{\text {, B. B. Schrank }}{ }^{3}$, K. Brigatti ${ }^{4}$, C. Gonzaga-Jauregui ${ }^{5}$, E. Puffenberger ${ }^{4}$, G. Wunderlich ${ }^{6}$, P. De Jonghe ${ }^{2,7}$, T. Deconinck ${ }^{2}$, K. Strauss ${ }^{4}, J$. Baets ${ }^{2,7}$, B. Wirth $^{l}$

${ }^{1}$ Institute for Human Genetics, Centre for Molecular Medicine and Center for Rare Diseases, Cologne, Germany, ${ }^{2}$ Neurogenetics Group, Center for Molecular Neurology (VIB), Laboratory of Neuromuscular Pathology, Institute Born-Bunge, University of Antwerp, Antwerp, Belgium, ${ }^{3}$ DKD HELIOS Kliniken, Department of Neurology, Wiesbaden, Germany, ${ }^{4}$ Clinic for Special Children, Strasburg, PA, United States, ${ }^{5}$ Regeneron Genetics Center, Regeneron Pharmaceuticals Inc., Tarrytown, NY, United States, ${ }^{6}$ Department of Neurology, University Hospital Cologne, Cologne, Germany, ${ }^{7}$ Neuromuscular Reference Centre, Department of Neurology, Antwerp University Hospital, Antwerp, Belgium

Introduction: Distal hereditary motor neuropathies (dHMN) are heterogeneous diseases characterized by motor neuron degeneration and atrophy. 50\% of the individuals with dHMN have a mutation in a hitherto undiscovered gene.

Materials and Methods: WES/WGS to identify the dHMN-causative gene. Assessment of candidate variants consequences on splicing, protein expression, and subcellular localization, using various cellular systems.

Results: We identified de novo or inherited variants (c.2945G>A, p.C982Y; c.3410C >T, p.A1137V; c.4382G>A, p.R1461Q and c.3524G>A, p.W1175*) in GBF1 (Golgi Brefeldin A-Resistant Guanine Nucleotide Exchange Factor1) in four unrelated families with dominant or sporadic dHMN. Other known dHMN-implicated genes were excluded. Affected individuals show dHMN with slowly progressive distal muscle weakness and musculoskeletal deformities (scoliosis, pes cavus, and hammertoes). Electrophysiological studies confirmed chronic neurogenic changes with axonal involvement. Three patients had additionally distal sensory loss. GBF1 plays a pivotal role in Golgi maintenance and retrograde trafficking as well as in mitochondrial positioning and function. Immunostaining analyses revealed Golgi fragmentation in all patients' fibroblasts, being more severe in W1175*. Subtle mitochondrial defects, including reduced mitochondrial content and fragmentation, were observed in all fibroblast lines; except for A1137V. These defects are in line with co-immunoprecipitation studies in HEK293T cells that demonstrated reduced GBF1-Miro2 interaction in C982Y and R1461Q. None of the $G B F 1$ variants affects splicing.

Conclusion: Previous studies have linked Golgi fragmentation and mitochondrial fusion-fission defects with motor neuron degeneration and atrophy. Our results emphasize the role of GBF1 in these contexts and indicate that GBF1 mutations are a novel dHMN cause.

N. Mendoza Ferreira: None. M. Karakaya: None. N. Cengiz: None. D. Beijer: None. N. Fuhrmann: None. I. Hölker: None. B. Schrank: None. K. Brigatti: None. C. Gonzaga-Jauregui: None. E. Puffenberger: None. G. Wunderlich: None. P. De Jonghe: None. T. Deconinck: None. K. Strauss: None. J. Baets: None. B. Wirth: None.

\section{C20.3}

Biallelic JAM2 variants lead to early-onset recessive primary familial brain calcification

L. V. Schottlaender ${ }^{1,2,3^{*}}$, R. Abeti ${ }^{4}$, Z. Jaunmuktane $e^{4,5}$, C. Macmillan $^{6}$, V. Chelban ${ }^{2}$, B. O'Callaghan ${ }^{2}, J$. McKinley ${ }^{7,8}$, R. Maroofian ${ }^{2}$, S. Efthymiou ${ }^{2}$, A. Fragkou ${ }^{2}$, R. Forbes ${ }^{9}, M$. Soutar $^{10}$, J. Livingston ${ }^{11}$, B. Kalmar ${ }^{2}$, O. Swayne ${ }^{12}$, G. Hotton $^{12}$, A. Pittman ${ }^{2}$, J. Mendes de Oliveira ${ }^{13}$, M. de Grandis $^{14}$, A. Richard-Loendt ${ }^{10}, F$. Launchbury ${ }^{10}, J$. Althonayan $^{4}$, G. McDonnell ${ }^{8}$, A. Carr ${ }^{2,12}$, S. Khan ${ }^{15}$, C. Beetz $^{15}$, A. Bisgin ${ }^{16}$, S. Tug Bozdogan ${ }^{16}$, A. Begtrup ${ }^{17}$, E. Torti $^{17}$, L. Greensmith ${ }^{2}$, P. Giunti ${ }^{4}$, P. Morrison ${ }^{18}$, S. Brandner $^{5}$, M. Aurrand-Lions ${ }^{14}$, H. Houlden ${ }^{2,12}$

${ }^{1}$ FLENI Neurological Research Institute, Capital federal, Argentina, ${ }^{2}$ Department of Neuromuscular Diseases, UCL Queen Square Institute of Neurology, Queen Square, UK, London, United Kingdom, ${ }^{3}$ Dubowitz Neuromuscular Centre, UCL Great Ormond Street Institute of Child Health, London, United Kingdom, ${ }^{4}$ Department of Clinical and Movement Neurosciences, UCL Queen Square Institute of Neurology, London, United Kingdom, ${ }^{5}$ Division of Neuropathology, The National Hospital for Neurology and Neurosurgery, University College London Hospitals NHS Foundation Trust, London, United Kingdom, 
${ }^{6}$ Department of Pediatrics, University of Chicago, Chicago, IL, United States, ${ }^{7}$ Department of Neurology, Dublin Neurological Institute at the Mater Misericordiae University Hospital, Dublin, Ireland, ${ }^{8}$ Regional Neurosciences Centre, Royal Victoria Hospital, Belfast, United Kingdom, ${ }^{9}$ Neurology Centre, Southern HSC Trust, Craigavon Area Hospital, Portadown, United Kingdom, ${ }^{10}$ Department of Neurodegenerative Disease, UCL Queen Square Institute of Neurology, London, United Kingdom, ${ }^{11}$ Paediatric Neurology, The Leeds Teaching Hospitals NHS Trust, Leeds General Infirmary, Leeds, United Kingdom, ${ }^{12}$ The National Hospital for Neurology and Neurosurgery, Queen Square, London, United Kingdom, ${ }^{13}$ Universidade Federal de Pernambuco, Departamento de Neuropsiquiatria, Recife, Brazil, ${ }^{14}$ Aix-Marseille University, Inserm, CNRS, Institut Paoli-Calmettes, CRCM, Marseille, France, ${ }^{15}$ CENTOGENE AG, Rostock, Germany, ${ }^{16}$ Medical Genetics Department of Medical Faculty \& AGENTEM (Adana Genetic Diseases Diagnosis and Treatment Center), Çukurova University, Adana, Turkey, ${ }^{17}$ GeneDx, 207 Perry Parkway, Gaithersburg, MD, United States, ${ }^{18}$ Centre for Cancer Research and Cell Biology, Queens University Belfast, Belfast, United Kingdom

Primary familial brain calcification (PFBC) is a rare neurodegenerative disorder characterised by a combination of neurological, psychiatric and cognitive decline associated with calcium deposition on brain imaging. To date, mutations in five genes have been linked to PFBC. However, over $50 \%$ of individuals affected by PFBC have no molecular diagnosis. We report four unrelated families presenting with initial learning difficulties and seizures and later psychiatric symptoms, cerebellar ataxia, extrapyramidal signs and extensive calcifications on brain imaging. Through a combination of homozygosity mapping and exome sequencing, we mapped this phenotype to chromosome 21q21.3 and identified biallelic variants in JAM2. $J A M 2$ encodes for the junctional-adhesion-molecule-2, a key tight-junction protein in blood-brain-barrier permeability. We show that JAM2 variants lead to reduction of $J A M 2$ mRNA expression and absence of JAM2 protein in patient's fibroblasts, consistent with a loss-of-function mechanism. We show that the human phenotype is replicated in the jam2 complete knockout mouse (jam2 $\mathrm{KO}$ ). Furthermore, neuropathology of jam2 KO mouse showed prominent vacuolation in the cerebral cortex, thalamus, cerebellum and particularly widespread vacuolation in the midbrain with reactive astrogliosis and neuronal density reduction. The regions of the human brain affected on neuroimaging are similar to the affected brain areas in the myorg PFBC null mouse. Along with JAM3 and OCLN, $J A M 2$ is the third tight-junction gene in which biallelic variants are associated with brain calcification, suggesting that defective cell-to-cell adhesion and dysfunction of the movement of solutes through the paracellular spaces in the neurovascular unit is a key mechanism in CNS calcification.

L. V. Schottlaender: None. R. Abeti: None. Z. Jaunmuktane: None. C. Macmillan: None. V. Chelban: None. B. O'Callaghan: None. J. McKinley: None. R. Maroofian: None. S. Efthymiou: None. A. Fragkou: None. R. Forbes: None. M. Soutar: None. J. Livingston: None. B. Kalmar: None. O. Swayne: None. G. Hotton: None. A. Pittman: None. J. Mendes de Oliveira: None. M. de Grandis: None. A. Richard-Loendt: None. F. Launchbury: None. J. Althonayan: None. G. McDonnell: None. A. Carr: None. S. Khan: A. Employment (full or part-time); Significant; CENTOGENE. C. Beetz: A. Employment (full or parttime); Significant; CENTOGENE. A. Bisgin: None. S. Tug Bozdogan: None. A. Begtrup: A. Employment (full or parttime); Significant; Genedx. E. Torti: A. Employment (full or part-time); Significant; Genedx. L. Greensmith: None. P. Giunti: None. P. Morrison: None. S. Brandner: None. M. Aurrand-Lions: None. H. Houlden: None.

\section{C20.4}

Loss of UGP2 in brain leads to a severe epileptic encephalopathy, emphasizing that bi-allelic isoform specific start-loss mutations of essential genes can cause genetic diseases

E. Perenthaler ${ }^{1 *}$, A. Nikoncuk ${ }^{l}$, S. Yousefi ${ }^{l}$, W. M. Berdowski ${ }^{l}$, M. Alsagob ${ }^{2}$, I. Capo ${ }^{3}$, H. C. van der Linde ${ }^{I}, P$. van den Berg' ${ }^{1}$, E. H. Jacobs' ${ }^{1}$, D. Putarl, M. Ghazvini', E. Aronica $^{4}$, W. F. J. van IJcken ${ }^{l}$, W. G. de Valk', E. Medici-van den Herik ${ }^{1}$, M. van Slegtenhorst ${ }^{1}$, L. Brick ${ }^{5}, M$. Kozenko $^{5}$, J. N. Kohler ${ }^{6}$, J. A. Bernstein ${ }^{6}$, K. G. Monaghan ${ }^{7}$, A. Begtrup ${ }^{7}$, R. Torene ${ }^{7}$, A. Al Futaisi ${ }^{8}, F$. Al Murshedi ${ }^{9}, F$. Al Azri ${ }^{9}$, E. Kamsteeg ${ }^{10}$, M. Mojarrad ${ }^{11}$, A. Eslahi ${ }^{11}$, E. Ghayoor Karimiani ${ }^{12}$, J. Vandrovcova ${ }^{13}, \mathrm{~F}_{\text {. Zafar }}{ }^{14}, \mathrm{~N}$. Rana $^{14}$, K. K. Kandaswamy ${ }^{15}$, J. Hertecant ${ }^{16}$, P. Bauer ${ }^{15}$, D. Colak ${ }^{2}$, S. Efthymiou ${ }^{13}$, H. Houlden ${ }^{13}$, A. M. BertoliAvella $^{15}$, R. Maroofian ${ }^{13}$, K. Retterer ${ }^{7}$, A. C. Brooks ${ }^{1}, N$. Kaya $^{2}$, T. J. van Ham ${ }^{l}$, T. Barakat ${ }^{1}$

${ }^{1}$ Erasmus MC, Rotterdam, Netherlands, ${ }^{2}$ King Faisal Specialist Hospital and Research Centre, Riyadh, Saudi Arabia, ${ }^{3}$ Faculty of Medicine Novi Sad, Novi Sad, Serbia, ${ }^{4}$ Amsterdam UMC, Amsterdam, Netherlands, ${ }^{5}$ McMaster Children's Hospital, Hamilton, ON, Canada, ${ }^{6}$ Stanford University School of Medicine, Stanford, CA, United States, ${ }^{7}$ GeneDX, Gaithersburg, MD, United States, ${ }^{8}$ Sultan Qaboos University, Muscat, Oman, ${ }^{9}$ Sultan Qaboos University Hospital, Muscat, Oman, ${ }^{10}$ Radboud University, Nijmegen, Netherlands, ${ }^{11}$ Mashhad University of Medical Sciences, Mashhad, Iran, Islamic Republic of, ${ }^{12} \mathrm{St}$. George's University, London, United Kingdom, ${ }^{13} \mathrm{UCL}$ 
Queen Square Institute of Neurology, London, United Kingdom, ${ }^{14}$ Children's hospital and institute of Child health, Multan, Pakistan, ${ }^{15}$ CENTOGENE AG, Rostock, Germany, ${ }^{16}$ UAE University, Al-Ain, United Arab Emirates

Developmental and/or epileptic encephalopathies (DEEs) are a group of devastating genetic disorders, resulting in early onset, therapy resistant seizures and developmental delay. Here we report on 19 individuals from 12 families presenting with a severe form of intractable epilepsy, severe developmental delay, progressive microcephaly and visual disturbance. Whole exome sequencing identified a recurrent, homozygous variant $(\operatorname{chr} 2: 64083454 \mathrm{~A}>\mathrm{G})$ in the essential UDP-glucose pyrophosphorylase (UGP2) gene in all probands. This rare variant results in a tolerable Met12Val missense change of the longer UGP2 protein isoform but causes a disruption of the start codon of the shorter isoform. We show that the absence of the shorter isoform leads to a reduction of functional UGP2 enzyme in brain cell types, leading to altered glycogen metabolism, upregulated unfolded protein response and premature neuronal differentiation, as modelled during pluripotent stem cell differentiation in vitro. In contrast, the complete lack of all UGP2 isoforms leads to differentiation defects in multiple lineages in human cells. Reduced expression of Ugp2a/ Ugp2b in vivo in zebrafish mimics visual disturbance and mutant animals show a behavioral phenotype. Our study identifies a recurrent start codon mutation in $U G P 2$ as a cause of a novel autosomal recessive DEE. Importantly, it also shows that isoform specific start-loss mutations causing expression loss of a tissue relevant isoform of an essential protein can cause a genetic disease, even when an organismwide protein absence is incompatible with life. We provide additional examples where a similar disease mechanism applies.

E. Perenthaler: None. A. Nikoncuk: None. S. Yousefi: None. W. M. Berdowski: None. M. Alsagob: None. I. Capo: None. H. C. van der Linde: None. P. van den Berg: None. E. H. Jacobs: None. D. Putar: None. M. Ghazvini: None. E. Aronica: None. W. F. J. van IJcken: None. W. G. de Valk: None. E. Medici-van den Herik: None. M. van Slegtenhorst: None. L. Brick: None. M. Kozenko: None. J. N. Kohler: None. J. A. Bernstein: None. K. G. Monaghan: A. Employment (full or part-time); Significant; GeneDX. A. Begtrup: A. Employment (full or part-time); Significant; GeneDX. R. Torene: A. Employment (full or part-time); Significant; GeneDX. A. Al Futaisi: None. F. Al Murshedi: None. F. Al Azri: None. E. Kamsteeg: None. M. Mojarrad: None. A. Eslahi: None. E. Ghayoor Karimiani: None. J. Vandrovcova: None. F. Zafar: None. N. Rana: None. K. K. Kandaswamy: A. Employment (full or part-time); Significant; CENTOGENE AG. J. Hertecant: None. P. Bauer: A. Employment (full or part-time); Significant; CENTOGENE
AG. D. Colak: None. S. Efthymiou: None. H. Houlden: None. A. M. Bertoli-Avella: A. Employment (full or parttime); Significant; CENTOGENE AG. R. Maroofian: None. K. Retterer: A. Employment (full or part-time); Significant; GeneDX. A. C. Brooks: None. N. Kaya: None. T. J. van Ham: None. T. Barakat: None.

\section{C20.5}

Human-lineage-specific genomic elements are enriched within genes implicated in neurodegenerative diseases

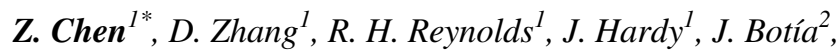
S. A. Gagliano Taliun ${ }^{3}$, M. Ryten ${ }^{1}$

${ }^{I}$ Department of Neurodegenerative Diseases, UCL Queen Square Institute of Neurology, London, United Kingdom, ${ }^{2}$ Departamento de Ingeniería de la Información y las Comunicaciones. Universidad de Murcia, Murcia, Spain, ${ }^{3}$ Center for Statistical Genetics and Department of Biostatistics, University of Michigan, Ann Arbor, MI, United States

Introduction: While encephalization has had advantages for humans, this process may predispose us to neurodegenerative disorders including Alzheimer's and Parkinson's diseases, which do not occur naturally in aged non-human primates. This suggests that genomic features specific to the human lineage may provide insights into brain-related diseases.

Methods: In this study, we leverage high-depth whole genome sequencing data from $\sim 8,000$ individuals and improved conservation metrics to generate a combined annotation identifying regions simultaneously depleted for genetic variation (constrained regions) and have low conservation at ten base pair resolution. We test our proposal that these constrained, non-conserved regions (CNCRs) result from human-specific purifying selection and are enriched for neurological disease risk loci.

Results: We find that CNCRs are significantly depleted from protein-coding genes but enriched within IncRNAs. We use stratified-linkage disequilibrium score regression to demonstrate that CNCRs are enriched for per-SNP heritability of complex human-specific phenotypes, namely intelligence (coefficient $\mathrm{p}=1.4 \mathrm{e}-3$ ). We find that high CNCR density is a feature of genes already implicated in neurological diseases, including MAPT and APOE. Focusing on $A P O E$, we identify high CNCR density within an unannotated intron-3 retaining non-coding transcript reported to regulate neuronal $A P O E$. Using human frontal cortex RNA-sequencing data, we find the proportion of the intron-3-retaining transcript is higher in Alzheimer's disease than control participants $(\mathrm{p}<2.2 \mathrm{e}-16)$ and associated with 
more severe Braak pathology (adjusted $\mathrm{r}^{\wedge} 2=0.6778, \mathrm{p}<$ 2.2e-16).

Conclusions: We generate a highly-granular annotation that supports the importance of human-lineage-specific sequences in neurodegenerative disorders with implications for disease modelling and interpretation of non-coding variants.

\section{ZC: LWENC Doctoral-Funding}

Z. Chen: None. D. Zhang: None. R. H. Reynolds: None. J. Hardy: None. J. Botía: None. S. A. Gagliano Taliun: None. M. Ryten: None.

\section{C20.6}

Full-length RNA sequencing of Alzheimer brain sample using long reads reveals complex alternative splicing patterns

\section{J. Ekholm* , E. Tseng, T. Hon, J. Underwood}

\section{Pacific Biosciences, Menlo Park, CA, United States}

Long-read mRNA sequencing such as PacBio's Iso-Seq method offer high-throughput transcriptome profiling that circumvents the transcript assembly problem by sequencing full-length cDNA. The Iso-Seq method has emerged as the most reliable technology for fully characterizing isoforms and, in turn, help shed light on underlying disease mechanisms. Here, we have utilized the Iso-Seq method to sequence an Alzheimer's disease whole brain sample. This is a devastating neurodegenerative disease that affects $\sim 44$ million people worldwide, making it the most common form of dementia. Studies looking into disease mechanism have shown that changes in gene expression due to alternative splicing likely contribute to the initiation and progression of Alzheimer's disease. Hence, efforts have been made to better understand the gene expression changes in the Alzheimer brain by sequencing the transcriptome of affected brain regions. In this study, complex alternative splicing patterns emerged, with 162,290 unique transcripts up to $14 \mathrm{~kb}$ in length from 17,670 genes being detected. More than $60 \%$ of these transcripts were novel isoforms, the vast majority of which have supporting cage peak data and polyadenylation signals. To further investigate the sample, we also applied the Iso-Seq solution to single cell RNA samples. In this experiment we obtained between 150,000180,000 unique isoforms from 15,000-17,000 genes. Over $50 \%$ of the isoforms categorized as novel isoforms, suggesting a high degree of undiscovered transcripts in the human samples, demonstrating the utility of long-read RNA sequencing for human disease research.

J. Ekholm: A. Employment (full or part-time); Significant; PacBio. E. Ownership Interest (stock, stock options, patent or other intellectual property); Significant; PacBio. E.
Tseng: A. Employment (full or part-time); Significant; PacBio. E. Ownership Interest (stock, stock options, patent or other intellectual property); Significant; PacBio. T. Hon: A. Employment (full or part-time); Significant; PacBio. E. Ownership Interest (stock, stock options, patent or other intellectual property); Significant; PacBio. J. Underwood: A. Employment (full or part-time); Significant; PacBio. E. Ownership Interest (stock, stock options, patent or other intellectual property); Significant; PacBio.

\section{C21 Methods and Applications of Association Studies in Large Cohorts}

\section{C21.1}

A genome-wide association study of sex at birth in 3 million individuals reveals widespread sex-differential participation bias with potential implications for GWAS interpretation

N. Piratsu ${ }^{1}$, M. Cordioli ${ }^{2}$, G. Mignogna ${ }^{3}$, A. Abdellaoui $^{4}$, P. Nandakumar $^{5}$, B. Hollis ${ }^{6}$, M. Kanai ${ }^{7}$, V. Manikandan ${ }^{8}$, P. Della Briotta Parolo ${ }^{2}$, N. Baya ${ }^{7}$, C. Carey ${ }^{7}$, J. Karjalainen $^{2}$, T. D. Als ${ }^{8}$, M. D. Van der Zee, F. R. Day ${ }^{6}, K$. K. Ong ${ }^{6}, T$. Morisaki ${ }^{9}$, E. de Geus ${ }^{4}$, R. Bellocco ${ }^{3}, Y$. Okada ${ }^{9}, A$. Børglum ${ }^{8}$, P. Joshi ${ }^{1}$, A. Auton ${ }^{5}$, D. Hinds ${ }^{5}$, B. Neale ${ }^{7}$, R. Walters $^{7}$, Finngen Study, 23andMe Research Team, iPSYCH Consortium, M. G. Nivard ${ }^{4}$, J. R. B. Perry ${ }^{6}$, a. ganna $^{2 *}$

${ }^{1}$ University of Edinburgh, Edinburgh, United Kingdom, ${ }^{2}$ Institute for Molecular Medicine Finland, Helsinki, Finland, ${ }^{3}$ University of Milano Bicocca, Milan, Italy, ${ }^{4} V U$ amsterdam, Amsterdam, Netherlands, ${ }^{5} 23$ andMe Inc, Sunnyvale, CA, United States, ${ }^{6}$ University of Cambridge, Cambridge, United Kingdom, ${ }^{7}$ Broad Institute, Cambridge, MA, United States, ${ }^{8}$ Aarhus university, Aarhus, Denmark, ${ }^{9}$ University of Tokyo, Tokyo, Japan

Genetic association results are often interpreted with the assumption that factors influencing voluntary participation do not affect downstream analyses. Understanding the genetic basis of this participation bias is challenging as it involves the genotypes of unseen individuals. As a proof of concept, we demonstrate that sex at birth becomes a heritable trait in the presence of sex-differential participation bias. By performing a GWAS of sex in $\sim 3.3 \mathrm{M}$ men and women, we identify over 150 genome-wide significant loci which highlight complex traits underpinning differences in study participation between sexes. For example, body mass index (BMI) raising alleles were observed at higher frequency in men compared to women, suggesting that genetically higher-weight women are less likely to participate in population studies than men. We demonstrate how 
these biases can lead to incorrect inferences in Mendelian Randomization analyses and propose a conceptual framework for addressing these biases. Our findings highlight a new dimension of challenges genetic studies may begin to face as sample sizes continue to grow.

N. Piratsu: None. M. Cordioli: None. G. Mignogna: None. A. Abdellaoui: None. P. Nandakumar: A. Employment (full or part-time); Significant; 23andMe Inc. B. Hollis: None. M. Kanai: None. V. Manikandan: None. P. Della Briotta Parolo: None. N. Baya: None. C. Carey: None. J. Karjalainen: None. T. D. Als: None. M. D. Van der Zee: None. F. R. Day: None. K. K. Ong: None. T. Morisaki: None. E. de Geus: None. R. Bellocco: None. Y. Okada: None. A. Børglum: None. P. Joshi: None. A. Auton: A. Employment (full or part-time); Significant; 23andMe Inc. D. Hinds: A. Employment (full or part-time); Significant; 23andMe Inc. B. Neale: None. R. Walters: None. M. G. Nivard: None. J. R. B. Perry: None. A. ganna: None.

\section{C21.2}

\section{A phenome-wide gene-based collapsing analysis of rare loss-of-function and missense variation in $\mathbf{2 6 8 , 4 5 1}$ UK Biobank exomes}

\section{Q. Wang, K. Carss, K. Smith, S. Petrovski*}

Centre for Genomics Research (CGR), Discovery Sciences, BioPharmaceuticals R\&D, AstraZeneca, Cambridge, United Kingdom

Historically, the genetic basis of complex disease has been investigated by genome-wide association studies using genotyping arrays. This approach is cost efficient but limited to known and common variants. Via advanced access to 300K UK Biobank participant exomes, we studied the rare protein-coding variation contribution to thousands of phenotypic endpoints. Containing clinical, biomarker, and other phenotypic information, this resource is unique in breadth and depth.

We performed collapsing analyses considering a range of genetic architectures. In total, 12 classes of qualifying variants were studied according to predicted protein effect, allele frequency, and zygosity. We expanded our scope beyond loss-of-function (LoF) variation with models targeting missense variation predicted to be deleterious or located in regions of missense tolerance ratio (MTR) constraint. A synonymous genetic model was used to define our empirical null p-value distribution.

Using exomes from 268,451 individuals, we performed a PheWAS studying the relationships between $\sim 19 \mathrm{~K}$ genes and $\sim 15 \mathrm{~K}$ phenotypes. Our cloud-based platform delivered $>2.5$ billion analyses within 24 hours. Among study-wide significant findings $68 \%$ are previously known, $17 \%$ are known disease genes but novel phenotype associations, and $15 \%$ are novel disease genes. We uncovered associations driven by missense variants clustered in genic regions that were missed when missense constraint was not considered. Some of these were not captured by a LoF model, suggesting possible gain-of-function signals.

We will illustrate AstraZeneca's use of these data to rapidly validate and inform safety profiles of potential drug targets, identify novel targets, predict drug repositioning opportunities, and study prevalence of molecular diagnoses.

Q. Wang: A. Employment (full or part-time); Significant; AstraZeneca. K. Carss: A. Employment (full or part-time); Significant; AstraZeneca. K. Smith: A. Employment (full or part-time); Significant; AstraZeneca. S. Petrovski: A. Employment (full or part-time); Significant; AstraZeneca.

\section{C21.3}

FinnGen - study at its midway: already identifying dozens of novel variants for tens of diseases and traits

\section{Perola ${ }^{1,2,3 *}$}

${ }^{I}$ Finnish Institute for Health and Welfare (THL), Helsinki, Finland, ${ }^{2}$ University of Helsinki, Helsinki, Finland, ${ }^{3}$ On behalf of the FinnGen Study, Helsinki, Finland

The FinnGen project is a public-private partnership study in Finland designed to benefit from the genetic isolation of Finnish population to identify variants contributing to common and rare diseases. All Finnish biobanks across the country participate in the study. The final aim is to collect more than $10 \%$ of the adult population of Finland, e.g. about 500.000 participants, and utilize the well validated highquality, lifelong health registry data for a GWAS-PheWas setting. To date, after two and a half years of recruitment, we have GWAS genotyped 224578 individuals and analysed this data against 3882 endpoints and 84 million health care events. The current number of biobanked participants for the project is already $>340000$. Previously, we have shown that the average Finn has more low-frequency loss-of-function variants and complete gene knockouts as non-Finn Europeans. Following this, now in FinnGen we have so far identified more than 400 genome-wide significant loci (about 350 novel), which have a Finnish enriched, low frequency $(\mathrm{MAF}<0.1$, enrichment $>1.5)$ variant. More than 60 of these loci have a missense or protein truncating variant. The longitudinal setting and inclusion of the parental causeof-death are producing novel insights into aetiology and progression of hundreds of diseases. FinnGen first year summary results of 96,499 individuals from November 2018 are now publicly released at http://r2.finngen.fi/.

M. Perola: B. Research Grant (principal investigator, collaborator or consultant and pending grants as well as 
grants already received); Significant; Abbvie, Astra Zeneca, Biogen, Celgene, Genentech, GSK, Janssen Biotech, Maze Therapeutics, MSD, Pfizer, Sanofi.

\section{C21.4}

Discovery, estimation and prediction analysis using a Bayesian survival model for complex traits

\section{S. E. Ojave ${ }^{1 *}$, D. Trejo-Banos ${ }^{l}$, M. Patxot ${ }^{l}$, K. Fischer ${ }^{2}, A$. Kousathanas $^{1}$, M. R. Robinson ${ }^{3}$ \\ ${ }^{1}$ University of Lausanne, Lausanne, Switzerland, ${ }^{2}$ Univer- sity of Tartu, Tartu, Estonia, ${ }^{3}$ Institute of Science and Technology, Vienna, Austria}

Time-to-event analysis using genotypic data may enable a better understanding of the mechanisms and underlying genetic architecture behind the onset and development of common complex disease. Typically, time-to-event data are not normally distributed, are right censored and in genomic studies, the number of covariates strongly exceeds the number of observations. Previous work has not adequately addressed these issues, potentially leading to underpowered models and biased effect size estimates.

Here, we propose a hierarchical Bayesian model which assumes that time-to-event has a Weibull distribution, handles sparsity with spike and slab variable selection and variance partitioning, considers right censoring, and yields estimates of the proportion of genetic variance explained (SNP heritability). The model can handle datasets of hundreds of thousands of people and millions of genetic markers by combining synchronous parallel Gibbs sampling and adaptive rejection sampling.

In simulations, our approach outperformed previous approaches and provided better genetic effect estimation. We then applied our model to UK Biobank data for a widerange of traits, including time-to-death, time to cardiovascular disease and time-to-menopause. We achieved $68 \%$ higher SNP heritability estimates compared to previous results for both time-to-menopause and time-to-menarche (SNP heritabilities of 0.26 and 0.42 respectively).

Our general framework enables more accurate discovery and estimation of the survival-related genomic marker effects providing novel insight into the genetic architecture of any very large-scale time-to-event data, and unprecedented statistical power to predict risk for medically important traits.

This work was funded by an SNSF Eccellenza Grant (PCEGP3_181181) to M.R.R.

S. E. Ojavee: None. D. Trejo-Banos: None. M. Patxot: None. K. Fischer: None. A. Kousathanas: None. M. R. Robinson: None.

\section{C21.5}

Simultaneous estimation of bi-directional causal effects and heritable confounding from GWAS summary statistics

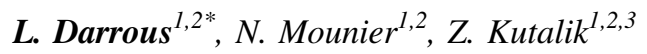

${ }^{1}$ University Center for Primary Care and Public Health, Lausanne, Switzerland, ${ }^{2}$ Swiss Institute of Bioinformatics, Lausanne, Switzerland, ${ }^{3}$ Genetics of Complex Traits, University of Exeter Medical School, Exeter, United Kingdom

Introduction: Mendelian Randomisation (MR), an increasingly popular method that estimates the causal effects of risk factors on complex human traits, has seen several extensions that relax its basic assumptions. However, most of these extensions suffer from two major limitations; under-exploitation of genome-wide markers, and sensitivity to the presence of a heritable confounder of the exposure-outcome relationship. To overcome these limitations, we propose a Latent Heritable Confounder MR (LHC-MR) method applicable to association summary statistics, which simultaneously estimates bi-directional causal effects, direct heritability, and confounder effects while accounting for sample overlap.

Results: We demonstrate that LHC-MR outperforms several existing MR methods in terms of bias and variance for a wide range of simulation settings and apply it to summary statistics of 13 complex traits. Besides several concordant results, LHC-MR unravelled new mechanisms (how being diagnosed for certain diseases might lead to improved lifestyle) and revealed potential false positive findings of standard MR methods (apparent causal effect of body mass index on educational attainment may be driven by a strong ignored confounder). Phenome-wide search to identify LHCimplied heritable confounders showed remarkable agreement between the LHC-estimated causal effects of the latent confounder and those for the potentially identified ones. Finally, LHC-MR naturally decomposes genetic correlation to causal effect-driven and confounder-driven contributions, demonstrating that the genetic correlation between systolic blood pressure and diabetes is predominantly confounderdriven.

Conclusion: LHC-MR, a structural equation mixed effect model, is a novel method that tackles both causal inference with increased precision and the genetic architecture of complex disease.

L. Darrous: None. N. Mounier: None. Z. Kutalik: None. 


\section{C21.6}

Multi-trait genome-wide association analysis of blood pressure identifies 45 additional loci

C. P. Cabrera ${ }^{1,2 *}$, R. Pazoki ${ }^{3,4,5}$, A. Giri $^{6}$, J. N. Hellwege ${ }^{7}$, E. Evangelou ${ }^{3,8}$, J. Ramírez ${ }^{1}$, L. V. Wain ${ }^{9,10}$, I. Tzoulaki ${ }^{3,8}$, T. L. Edwards ${ }^{11}$, P. Elliott ${ }^{3,12,13}$, P. B. Munroe ${ }^{1,2}, M . R$. Barnes $^{1,2}$, M. J. Caulfield ${ }^{1,2}, H$. R. Warren ${ }^{1,2}$, on behalf of the VA Million Veteran Program and the ICBP working group

${ }^{1}$ William Harvey Research Institute, Barts and The London School of Medicine and Dentistry, Queen Mary Univeristy of London, London, United Kingdom, ${ }^{2}$ National Institute for Health Research, Barts Cardiovascular Biomedical Research Center, Queen Mary University of London, London, United Kingdom, ${ }^{3}$ Department of Epidemiology and Biostatistics, Imperial College London, London, United Kingdom, ${ }^{4}$ Department of Life Sciences, College of Health and Life Sciences, Brunel University, London, United Kingdom, ${ }^{5}$ Centre for Inflammation Research and Translational Medicine, Brunel University, London, United Kingdom, ${ }^{6}$ Vanderbilt Genetics Institute,Vanderbilt Epidemiology Center, Department of Obstetrics and Gynecology,Vanderbilt University Medical Center, Nashville, TN, United States, ${ }^{7}$ Vanderbilt Genetics Institute, Division of Epidemiology, Department of Medicine, Institute for Medicine and Public Health,Vanderbilt University Medical Center, Nashville, TN, United States, ${ }^{8}$ Department of Hygiene and Epidemiology, University of Ioannina Medical School, Ioannina, Greece, ${ }^{9}$ Department of Health Sciences, University of Leicester, Leicester, United Kingdom, ${ }^{10}$ NIHR Leicester Biomedical Research Centre, Glenfield Hospital, Leicester, United Kingdom, ${ }^{11}$ Vanderbilt Genetics Institute, Division of Epidemiology, Department of Medicine, Institute for Medicine and Public Health, Vanderbilt University Medical Center, Nashville, TN, United States, ${ }^{12}$ National Institute for Health Research Imperial Biomedical Research Centre, Imperial College Healthcare NHS Trust and Imperial College London, London, United Kingdom, ${ }^{13}$ UK Dementia Research Institute, Imperial College London, London, United Kingdom

Introduction: Single-trait genome wide association studies (GWAS) have revealed over 1,000 blood pressure (BP) loci. However, these loci only account for less than one third of the BP genetic variation. Multi-trait GWAS is reported to increase discovery power by jointly analysing highly correlated traits. By performing the first large-scale multi-trait BP GWAS, we aimed 1) to compare multi-trait vs singletrait results and 2) identify additional loci.
Methods: We apply Multi-Trait Analysis of GWAS (MTAG) software to jointly analyse systolic BP, diastolic $\mathrm{BP}$ and pulse pressure traits using our recent GWAS discovery results from $\sim 750 \mathrm{k}$ individuals of European ancestry from UK Biobank and the International Consortium of Blood Pressure. To detect additional loci we tested $\sim 7$ million imputed genetic variants applying the same combined 1-stage and 2-stage design criteria as in the original GWAS, with replication using MTAG results from the US Million Veteran Program (n 220k).

Results: Single-trait GWAS yielded a higher number of significant independent signals genome-wide. Nevertheless, our multi-trait analysis identified 45 new BP loci that were not detected in the equivalent GWAS, of which nine remain novel (based on further BP loci discoveries since 2018).

Conclusions: Our multi-trait GWAS discovered additional BP loci. However, our results illustrate that the benefits of MTAG are trait-specific, requiring high pairwise correlation between all pairs of traits, and that more power is gained when MTAG is also used for meta-analysis of traits from different samples. This suggests that future BP genetics discovery projects should focus efforts on larger meta-analyses including new cohorts.

C. P. Cabrera: None. R. Pazoki: None. A. Giri: None. J. N. Hellwege: None. E. Evangelou: None. J. Ramírez: None. L. V. Wain: None. I. Tzoulaki: None. T. L. Edwards: None. P. Elliott: None. P. B. Munroe: None. M. R. Barnes: None. M. J. Caulfield: None. H. R. Warren: None.

\section{C22 Immunology and Hematology}

\section{C22.1}

Newborn screening for severe combined immunodeficiency (SCID) using combined $\mathrm{T}$ cell reception excision circles (TREC)/kappa-deleting element recombination element (KREC) assays and next generation sequencing: diagnostic yield from the new established Swiss programme

\section{Elgizouli ${ }^{*}$, J. Pascal ${ }^{1}$, A. Bahr ${ }^{1}$, S. Prader ${ }^{2}$, D.} Drozdof ${ }^{2}$, J. Pachlopnik Schmid ${ }^{2}$, K. Steindl ${ }^{1}$, A. Rauch ${ }^{l}, J$. Reichenbach ${ }^{2}$

${ }^{1}$ Institute of Medical Genetics, University Zurich, Schlieren, Switzerland, ${ }^{2}$ Department of Immunology, University Children's Hospital Zurich, Zurich, Switzerland

Introduction: Infants with severe combined immunodeficiency (SCID), a fatal form of primary immunodeficiency in the absence of early definitive therapy, show an improved outcome if diagnosed and treated within the first few months of life, making the condition a suitable target for newborn screening. Country-wide newborn screening for 
SCID was initiated in Switzerland in January 2019 with about 80.000 live births per year.

Methods: DNA samples from dried blood spots of from all neonates born in Switzerland in 2019 were assayed for T-cell receptor excision circles (TREC) and kappa-deleting element recombination excision circles (KREC). Cases with low TREC and/or low KREC counts subsequently underwent clinical genetic assessment and whole exome sequencing (WES)-based mutation screening and/or highresolution chromosomal microarray testing. Children with dysmorphic features suspicious of DiGeorge syndrome were initially tested for the common $22 q 11.2$ microdeletion. In cases with suspected syndromes not detectable with WES, targeted Sanger sequencing was performed.

Results: Out of 15 neonates with low TREC/KREC counts identified through the screening procedure, we diagnosed 5 neonates with SCID and 6 with syndromic conditions in which a severe immunodeficiency constitutes a component of the clinical picture. Notably, 2 of the 6 syndromic immunodeficiencies were caused by RNAgenes and thus are not included in standard exome capture procedures.

Conclusion: Our findings support the utility of WES as an efficient and rapid mutation screening method in newborns with low TREC/KREC counts and suggest a Swiss incidence of SCID above the US incidence of 1 in 58,000 live births.

M. Elgizouli: None. J. Pascal: None. A. Bahr: None. S. Prader: None. D. Drozdof: None. J. Pachlopnik Schmid: None. K. Steindl: None. A. Rauch: None. J. Reichenbach: None.

\section{C22.2}

Strategies for increasing diagnostic rate in patients with primary immunological disorders within the Genomics England 100,000 Genomes Project

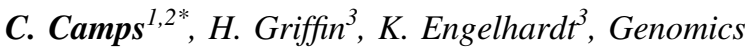 \\ England Research Consortium, S. Y. Patel ${ }^{2,4}$, S. \\ Hambleton $^{3,5}$, J. C. Taylor ${ }^{1,2}$
}

${ }^{1}$ Wellcome Centre for Human Genetics, University of Oxford, Oxford, United Kingdom, ${ }^{2}$ Oxford NIHR Biomedical Research Centre, Oxford, United Kingdom, ${ }^{3}$ Institute of Cellular Medicine, Newcastle University, Newcastle upon Tyne, United Kingdom, ${ }^{4}$ Department of Clinical Immunology, Oxford University Hospitals NHS Foundation Trust, Oxford, United Kingdom, ${ }^{5}$ Great North Children's Hospital, Newcastle upon Tyne Hospitals NHS Foundation Trust, Newcastle upon Tyne, United Kingdom

The UK's 100,000 Genomes Project has completed its sequencing and includes 74,180 rare disease genomes of which 2,005 are from patients or family members with a primary immunological diagnosis. Whilst the referring clinical centres review variants in known genes for a given patient's presenting condition, the Genomics England Clinical Interpretation partnerships (GECIPs) can investigate the pathogenesis of 'unsolved' cases, potentially leading to the identification of novel disease genes, phenotypes or mutation mechanisms. In the Immune GECIP we have taken a gene-focused approach to screen groups of subjects for whom no pathogenic mutation has yet been confirmed, which is more efficient than analysing results on a case-by-case basis. We started by screening known-disease genes $(n=482)$ for the presence of variants with high/moderate impact and frequency $\leq 1 \%$ in population databases across all probands. For each variant, we counted the number of homozygotes and heterozygotes among probands of the Immunology domain compared with the remaining probands, and further compared the groups at various levels to identify any enrichment. Results from this approach include i) identification of digenic cases among patients with complex phenotypes, ii) detection of immune disease-causing variants in patients outwith the Immunology domain, iii) detection of compound heterozygotes involving structural variants. This has paved the way for discovery of novel genes in the remaining patients while assisting in the timely delivery of clinically actionable findings, providing an exemplar of how the $100 \mathrm{~K}$ genomes dataset can be used to identify novel disease genes and mechanisms as well as refining disease entities.

C. Camps: None. H. Griffin: None. K. Engelhardt: None. S. Y. Patel: None. S. Hambleton: None. J. C. Taylor: None.

\section{C22.3}

Dysregulated expression of the long intergenic noncoding RNA (lincRNA), LINC01871, implicated in Sjögren's Syndrome pathogenesis

\section{L. Joachims ${ }^{l}$, B. Khatri ${ }^{l}$, K. L. Tessneer ${ }^{l}$, A. M. Stolarczyk ${ }^{l}$, G. B. Wiley ${ }^{l}$, A. Rasmussen ${ }^{1}$, J. M.

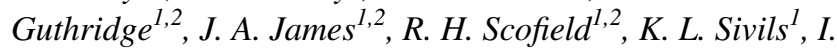 Adrianto $^{3}$, C. J. Lessard ${ }^{1,2 *}$ \\ ${ }^{1}$ OKLAHOMA MEDICAL RESEARCH FOUNDATION, OKLAHOMA CITY, OK, United States, ${ }^{2}$ University of Oklahoma Health Sciences Center, Oklahoma City, OK, United States, ${ }^{3}$ Henry Ford Health System, Detroit, MI, United States}

Introduction: Sjögren's syndrome (SS) is an autoimmune disease characterized by inflammatory destruction of exocrine glands. Here, we aimed to identify and characterize candidate lncRNAs with potential relevance to SS pathology. 
Results: RNA-seq was used on whole blood from SS patients $\left(\mathrm{n}=30\right.$ autoantibody negative $\left(\mathrm{SS}^{\mathrm{Ro}-}\right) ; \mathrm{n}=27$ autoantibody positive $\left(\mathrm{SS}^{\mathrm{Ro}+}\right)$ ) and healthy controls $(\mathrm{HC}, \mathrm{n}$ $=27)$ to identify differentially expressed (DE) lncRNAs (log2 fold change $(\mathrm{FC}) \geq 2$ or $\left.\leq 0.5 ; \mathrm{p}_{\mathrm{adj}}<0.05\right)$. We identified 1054 unique DE ncRNAs between $\mathrm{Ro}^{+}, \mathrm{Ro}^{-}$, and a combined analysis relative to $\mathrm{HC}$. Of these, 45 (1 lincRNA, 1 antisense, 43 pseudogenes) were overexpressed in all 3 SS subsets. To investigate the function of the novel lincRNA, LINC01871, we performed correlation analysis of the $\mathrm{SS}^{\text {Ro- }}$ transcriptome yielding several co-expressed protein coding RNAs involved in immune regulation (THEMIS, TBX21, ILIORA, IL2RB, etc.). Ingenuity Pathway Analysis of the SS and publicly available RNA expression data identified shared immunerelated pathways including cytotoxic $\mathrm{T}$ cell, NK cell, and $\mathrm{T}$ cell regulation. We used CRISPR editing to generate a single cell $\mathrm{LINCO} 871^{-/}$clone of HSB2 cells. RNA-seq of LINC01871 $1^{-\alpha}$ compared to unmodified HSB2 cells identified 1166 DE transcripts. Pathway analyses clustered DE transcripts into similar immune regulatory, cytotoxic and $\mathrm{T}$ cell pathways. Further, several $\mathrm{T}$ cell regulatory transcripts correlated with LINC01871 in $\mathrm{SS}^{\text {Ro- }}$ were downregulation after LINC01871 deletion: CD109 $\left(\mathrm{FC}=-9.7 ; \mathrm{p}_{\mathrm{adj}}=\right.$ $\left.5.3 \times 10^{-16}\right)$, IL22 $\left(\mathrm{FC}=-8.1 ; \mathrm{p}_{\mathrm{adj}}=7.6 \times 10^{-11}\right)$, PDCD1 $\left(\mathrm{FC}=-6.2 ; \mathrm{p}_{\mathrm{adj}}=1.1 \times 10^{-6}\right)$, THEMIS $\left(\mathrm{FC}=-3.8 ; \mathrm{p}_{\mathrm{adj}}=\right.$ $\left.2.7 \times 10^{-165}\right), T B X 21\left(\mathrm{FC}=-2.1 ; \mathrm{p}_{\mathrm{adj}}=3.3 \times 10^{-25}\right)$.

Conclusion: LINC01871 is a potential mediator of dysregulated $\mathrm{T}$ cell inflammatory pathways implicated in SS pathogenesis. Funding: NIGMS P30GM110766; Presbyterian Health Foundation

M. L. Joachims: None. B. Khatri: None. K. L. Tessneer: None. A. M. Stolarczyk: None. G. B. Wiley: None. A. Rasmussen: D. Speakers Bureau/Honoraria (speakers bureau, symposia, and expert witness); Modest; Novartis, ThermoFisher. J. M. Guthridge: B. Research Grant (principal investigator, collaborator or consultant and pending grants as well as grants already received); Modest; Dxterity. J. A. James: B. Research Grant (principal investigator, collaborator or consultant and pending grants as well as grants already received); Modest; Progentec Biosciences. F. Consultant/Advisory Board; Modest; Abbvie, Novartis, Jannsen. R. H. Scofield: B. Research Grant (principal investigator, collaborator or consultant and pending grants as well as grants already received); Modest; Pfizer. K. L. Sivils: None. I. Adrianto: None. C. J. Lessard: None.

\section{C22.4}

Clonal myelopoiesis in the UK biobank cohort: somatic ASXL1 mutations are strongly associated with smoking history

A. A. Z. Dawoud", W. J. Tapper, N. C. P. Cross

\section{Faculty of Medicine, Southampton, United Kingdom}

Age-related clonal hematopoiesis $(\mathrm{CH})$ is associated with the development of hematological malignancies and nonhematological disorders. We sought to determine the significance of myeloid $\mathrm{CH}$ in the UK Biobank cohort $(\mathrm{n}=502,524$, median age $=58$ years, median follow up $=10.6$ years $)$. Utilizing data from single nucleotide polymorphism arrays $(\mathrm{n}=486,941)$ and whole exome sequencing $(n=49,956)$, we identified $1166(0.24 \%)$ participants with myeloid $\mathrm{CH}$, defined by myeloid-associated mosaic chromosome abnormalities (mCA) and/or likely somatic driver mutations in DNMT3A, TET2, $A S X L 1, J A K 2, S R S F 2$ or PPM1D. The frequency of myeloid $\mathrm{CH}$ increased with age by approximately $10 \%$ per year $\left(\mathrm{OR}=1.10 ; \mathrm{P} \leq 2 \times 10^{-16}\right)$. Genome-wide association analysis identified two distinct signals within TERT that predisposed to $\mathrm{CH}$, plus a weaker signal corresponding to the JAK2 $46 / 1$ haplotype. $\mathrm{CH}$ and specific mutation types were associated with several blood features and clinical phenotypes, including an association between TET2 mutations and chronic obstructive pulmonary disease. Smoking history was significantly associated with $\mathrm{CH}$ : $53 \%(\mathrm{n}=622)$ of $\mathrm{CH}$ cases were past or current smokers compared to $44 \%(\mathrm{n}=13,651)$ of controls $\left(P=3.38 \times 10^{-6}\right)$, a difference principally due to current $\left(P=6.14 \times 10^{-6}, \quad \mathrm{OR}=1.09\right)$ rather than past smoking $(P=0.085)$. Strikingly, breakdown of $\mathrm{CH}$ by specific mutation type revealed that $A S X L 1$ loss of function mutations were most strongly associated with current smoking status $\left(P=1.92 \times 10^{-5}, \mathrm{OR}=1.07\right)$, and the only abnormality associated with past smoking $\left(P=2.60 \times 10^{-3}\right.$, $\mathrm{OR}=1.04)$. Indeed, $69 \%$ of participants with ASXL1 mutations $(n=69)$ were past or current smokers. We suggest that the inflammatory environment induced by smoking promotes the outgrowth of ASXL1-mutant clones.

A. A. Z. Dawoud: None. W. J. Tapper: None. N. C. P. Cross: None.

\section{C22.5}

A genome-wide meta-analysis yields 46 new loci associating with biomarkers of iron homeostasis

E. Ferkingstad ${ }^{1 *}$, M. K. Magnusson ${ }^{1,2}$, S. Bell ${ }^{3}$, A. S. Rigas $^{4}$, E. Allara ${ }^{3}$, G. Bjornsdottir ${ }^{1}$, A. Ramond ${ }^{3}$, E. Sørensen ${ }^{4,5}$, G. H. Halldorsson ${ }^{1}$, D. S. Paul ${ }^{3}$, K. S. Burgdorf ${ }^{4}$, H. P. Eggertsson ${ }^{1}$, S. Kristmundsdottir ${ }^{1}$, W. J. Astle $^{3}$, C. Erikstrup ${ }^{5}$, J. K. Sigurdsson ${ }^{1}$, D. Vuckovic ${ }^{3}$, V. Tragante $^{1}$, P. Surendran ${ }^{3}$, B. Vidarsson ${ }^{6}$, I. Jonsdottir $^{1,2}, T$. Hansen $^{7}$, O. Sigurdardottir ${ }^{8}$, H. Stefansson ${ }^{1}$, D. Rye ${ }^{9}$, DBDS Genomic Consortium, J. E. Peters ${ }^{3}$, D.

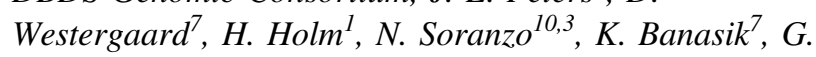
Thorleifsson $^{l}$, W. H. Ouwehand ${ }^{3}$, U. Thorsteinsdottir ${ }^{l}, P$. 
Sulem $^{l}$, A. S. Butterworth ${ }^{3}$, D. F. Gudbjartsson ${ }^{1,2}, J$. Danesh $^{3}$, S. Brunak ${ }^{11}$, E. Di Angelantonio ${ }^{3}$, H. Ullum $^{4}, K$. Stefansson ${ }^{1,2}$

${ }^{1}$ deCODE genetics/Amgen, Inc., Reykjavik, Iceland, ${ }^{2}$ University of Iceland, Reykjavik, Iceland, ${ }^{3}$ University of Cambridge, Cambridge, United Kingdom, ${ }^{4}$ Copenhagen University Hospital, Copenhagen, Denmark, ${ }^{5}$ Aarhus University Hospital, Aarhus, Denmark, ${ }^{6}$ The Laboratory in Mjodd, Reykjavik, Iceland, ${ }^{7}$ University of Copenhagen, Copenhagen, Denmark, ${ }^{8}$ Akureyri Hospital, Akureyri, Iceland, ${ }^{9}$ Emory University, Atlanta, GA, United States, ${ }^{10}$ Wellcome Sanger Institute, Cambridge, United Kingdom, ${ }^{11}$ Novo Nordisk Foundation Center for Protein Research, Copenhagen, Denmark

Iron deficiency is a major public health problem affecting maternal and child mortality, and iron overload has important health implications due to end-organ iron toxicity. In order to better understand the genetic regulation of iron homeostasis we carried out three genomewide association studies in Iceland, the UK and Denmark of blood levels of ferritin $(\mathrm{N}=246,139)$, total iron binding capacity $(\mathrm{N}=135,430)$, iron $(\mathrm{N}=163,511)$ and transferrin saturation $(\mathrm{N}=131,471)$, which are the biomarkers routinely measured for clinical assessment of iron status. In a meta-analysis we found associations with iron homeostasis biomarkers represented by 62 sequence variants at 56 loci. Fourteen of the variants associate with more than one biomarker, bringing the total number of observed associations to 87. Of the identified loci, 46 are novel and 10 are known. We assessed the association of these variants with proteomics data and various clinical endpoints. Five variants show sexual dimorphism: four have stronger effects in women while one has a stronger effect in men. The four variants with larger effects in women also have stronger effects in premenopausal than post-menopausal women, and their effect is likely working through blood loss and primarily menstrual bleeding. The associations implicate novel iron regulating proteins contributing to physiological processes involved in iron homeostasis: iron sensing and storage, inflammation, gut iron absorption, iron recycling, erythropoiesis and bleeding/menstruation. We furthermore identify both common and rare sequence variants contributing to risk of iron deficiency anemia, a major clinical entity that hitherto has not been studied thoroughly from a genetic point of view.

E. Ferkingstad: A. Employment (full or part-time); Significant; deCODE genetics/Amgen, Inc. M. K. Magnusson: A. Employment (full or part-time); Significant; deCODE genetics/Amgen, Inc. S. Bell: None. A. S. Rigas: None. E. Allara: None. G. Bjornsdottir: A. Employment (full or part-time); Significant; deCODE genetics/Amgen, Inc. A. Ramond: None. E. Sørensen: None. G. H. Halldorsson: A. Employment (full or part-time); Significant; deCODE genetics/Amgen, Inc. D. S. Paul: None. K. S. Burgdorf: None. H. P. Eggertsson: A. Employment (full or part-time); Significant; deCODE genetics/Amgen, Inc. S. Kristmundsdottir: A. Employment (full or part-time); Significant; deCODE genetics/Amgen, Inc. W. J. Astle: None. C. Erikstrup: None. J. K. Sigurdsson: A. Employment (full or part-time); Significant; deCODE genetics/ Amgen, Inc. D. Vuckovic: None. V. Tragante: A. Employment (full or part-time); Significant; deCODE genetics/ Amgen, Inc. P. Surendran: None. B. Vidarsson: None. I. Jonsdottir: A. Employment (full or part-time); Significant; deCODE genetics/Amgen, Inc. T. Hansen: None. O. Sigurdardottir: None. H. Stefansson: A. Employment (full or part-time); Significant; deCODE genetics/Amgen, Inc. D. Rye: None. J. E. Peters: None. D. Westergaard: None. H. Holm: A. Employment (full or part-time); Significant; deCODE genetics/Amgen, Inc. N. Soranzo: None. K. Banasik: None. G. Thorleifsson: A. Employment (full or part-time); Significant; deCODE genetics/Amgen, Inc. W. H. Ouwehand: None. U. Thorsteinsdottir: A. Employment (full or part-time); Significant; deCODE genetics/Amgen, Inc. P. Sulem: A. Employment (full or part-time); Significant; deCODE genetics/Amgen, Inc. A. S. Butterworth: None. D. F. Gudbjartsson: A. Employment (full or part-time); Significant; deCODE genetics/Amgen, Inc. J. Danesh: None. S. Brunak: None. E. Di Angelantonio: None. H. Ullum: None. K. Stefansson: A. Employment (full or part-time); Significant; deCODE genetics/Amgen, Inc.

\section{C22.6}

Identification of regulators of hematopoietic stem and progenitor cells in vivo in humans using population genetics

A. Lopez de Lapuente Portilla ${ }^{1 *}$, L. Ekhdal $^{l}$, C. Cafaro ${ }^{l}, Z$. Ali ${ }^{l}$, G. Thorleifsson ${ }^{2}, K$. Zemaitis ${ }^{1}, N$. Ugidos Damborienal, E. Johnsson $^{l}$, G. Norddahl ${ }^{2}, U$. Thorsteinsdottir $^{2}$, J. Larsson ${ }^{1}$, K. Stefansson ${ }^{2}$, B. Nilsson ${ }^{1}$

${ }^{1}$ Lund University, Lund, Sweden, ${ }^{2}$ deCODE Genetics, Reykjavik, Iceland

Introduction: Understanding how hematopoietic stem- and progenitor cells (HSPCs) are regulated is of central importance for the development of new therapies for blood disorders and for regenerative medicine. Traditionally, however, HSPC regulation has been studied in model systems, and little is known about the situation in vivo in humans. 
Methods: To learn how HSPCs are regulated under native conditions in humans, we carried out a first largescale genome-wide association study on $\mathrm{CD} 34^{+}$cells, representing HSPCs in blood. We used circulating CD34 ${ }^{+}$ levels as a proxy trait to expose regulators of key phenomena like HSPC pool size, migration, and early differentiation. We created a unique phenotyping platform based on high-throughput, high-resolution flow-cytometry and machine learning-based algorithms for automated flow data analysis, and quantified CD34 ${ }^{+}$cells in 9,936 adults.

Results: We identified 8 genome-wide $\left(P<5 \times 10^{-8}\right)$ and 20 suggestive loci $\left(P<5 \times 10^{-6}\right)$ associated with $\mathrm{CD} 34+$ levels. The two strongest were the HSPC migration receptor CXCR4 and a novel protein phosphatase never previously implicated in stem cell biology. Using eQTL, ATAC-seq, and promoter capture Hi-C analysis in isolated HSPCs, we pinpoint likely causal variants, including variants in distant regulatory elements selectively active in specific HSPC subpopulations. Furthermore, shRNA knockdown in primary $\mathrm{CD} 34^{+}$cells supports that some of the identified genes affect $\mathrm{CD} 34^{+}$proliferation and differentiation.

Conclusions: We report the first large-scale analysis of the genetic architecture of HSPC regulation, with potential implications for stem cell transplantation and the treatment of hematologic malignancies.

Grant information: European Research Council, Swedish Research Council, Swedish Cancer Society.

A. Lopez de Lapuente Portilla: None. L. Ekhdal: None. C. Cafaro: None. Z. Ali: None. G. Thorleifsson: A. Employment (full or part-time); Modest; deCODE Genetics. K. Zemaitis: None. N. Ugidos Damboriena: None. E. Johnsson: None. G. Norddahl: A. Employment (full or part-time); Modest; deCODE Genetics. U. Thorsteinsdottir: A. Employment (full or part-time); Modest; deCODE Genetics. J. Larsson: None. K. Stefansson: E. Ownership Interest (stock, stock options, patent or other intellectual property); Modest; deCODE Genetics. B. Nilsson: None.

\section{C23 Counselling, Communication and Deliberation}

\section{C23.1}

Helping very young children understand inherited cancer predisposition syndromes using bibliotherapy

\section{G. Schlub $^{1 *}$, A. Crook ${ }^{2}, J$. Fleming $^{l}$, K. Barlow-Stewart ${ }^{l}, J$. Kirk $^{3}$, K. Tucker ${ }^{4}$, S. Greening ${ }^{5}$}

${ }^{1}$ University of Sydney, Faculty of Health and Medicine, Sydney, Australia, ${ }^{2}$ Cancer Services, Royal North Shore Hospital, Sydney, Australia, ${ }^{3}$ Crown Princess Mary Cancer Centre, Westmead Hospital, Sydney, Australia, ${ }^{4}$ Hereditary Cancer Clinic, Prince of Wales Hospital, Sydney, Australia,
${ }^{5}$ Illawara Cancer Care Centre, Wollongong, Sydney, Australia

Introduction: Communication with children about hereditary conditions is difficult for parents. Yet, good communication strategies are leading determinants of adaptation and resilience. With inherited cancer predisposition syndromes that can affect very young children e.g. Li-Fraumeni Syndrome (LFS) and Hereditary Phaeochromocytoma and Paraganglioma Syndrome (HPPS), genetic testing and subsequent surveillance in at-risk children is the optimal intervention. Given this often commences early, providing these children and their parents appropriate genetic counselling and communication strategies is important. By adopting a bibliotherapeutic frameworkwhere stories are delivered prescriptively (i.e. 'bibliotherapy'), we developed and evaluated the first psychoeducational resource in Australia for children at risk of LFS and HPPS.

Materials and Methods: Illustrated storybooks for children aged 5-10 years old at risk of either LFS or HPPS were created based on models of developmental comprehension. Parents were invited to participate in the study and read the relevant storybook to their child/ren. Semistructured qualitative interviews were conducted and analysed thematically using a general inductive approach to ascertain parents' experience reading this storybook with their children.

Results and Conclusion: Twelve parents were interviewed (total 29 children). Unanimously, parents reported this resource would have been a useful communication tool had it been available when their children were first tested and/or diagnosed. Parents reported that the resource supported them with communication without raising emotional distress. We anticipate that by incorporating parental views, this cost-effective and easily deployable resource will have psycho-educational benefit and will improve the process of communication between parents and children about paediatric inherited cancer syndromes.

G. Schlub: None. A. Crook: None. J. Fleming: None. K. Barlow-Stewart: None. J. Kirk: None. K. Tucker: None. S. Greening: None.

\section{C23.2}

Towards personalized genetic counselling: exploring subgroups among counselees based on different facets of empowerment before the first visit

\author{
J. Voorwinden ${ }^{1 *}$, E. Birnie ${ }^{1}$, M. Plantinga ${ }^{l}, M$. Ausems $^{2}, N$. \\ Knoers $^{l}$, M. Velthuizen ${ }^{2}$, A. Lucassen ${ }^{3}$, I. van Langen ${ }^{1}$, A. \\ Ranchor $^{1}$
}


${ }^{1} U M C G$, Groningen, Netherlands, ${ }^{2} U M C U$, Utrecht, Netherlands, ${ }^{3}$ University of Southampton, Southampton, United Kingdom

Introduction: The main goal of genetic counselling is improving empowerment (cognitive/affective outcomes). The aim of this study is to explore subgroups among counselees based on different facets of empowerment. Subsequently, to examine if these subgroups are associated with socio-demographic and clinical variables. Recognizing the specific needs regarding genetic counselling for each subgroup, could facilitate personalized genetic counselling.

Materials and Methods: Data from 2446 unselected counselees from two Dutch academic centres was collected with a validated questionnaire: the GCOS-18, which measures six facets of empowerment (hope, knowledge about the condition, knowledge about genetic services, negative emotions, uncertainty about heredity, uncertainty about the treatment). Subgroups were analysed with latent profile analysis.

Results: Six subgroups could be distinguished. Profile 1 $(n=727)$ scored best on all facets of empowerment, and consisted of more higher educated and pre-symptomatic counselees. Profile $2(n=724)$ scored average on all facets of empowerment. Profile $3(n=225)$ experienced less hope, and consisted of more index patients. Profile $4(n=130)$ experienced less hope and knowledge; parents of referred children were overrepresented. Profile $5(n=491)$ had strong uncertainty about heredity, these counselees were older and more often at risk of hereditary cancer. Profile 6 $(n=149)$ had strong negative emotions, uncertainty about heredity and uncertainty about the treatment. These counselees were older and lower educated.

Conclusion: This study reveals subgroups among counselees. The scoring on different facets of empowerment allows to distinguish specific subgroups, each having their own specific needs, which could be used for personalization of the genetic counselling process.

J. Voorwinden: None. E. Birnie: None. M. Plantinga: None. M. Ausems: None. N. Knoers: None. M. Velthuizen: None. A. Lucassen: None. I. van Langen: None. A. Ranchor: None.

\section{C23.3}

Exploring patient deliberation prior to predictive genetic testing in the absence of immediate clinical utility

L. M. Ballard ${ }^{1 *}$, S. Doheny ${ }^{2}$, A. M. Lucassen ${ }^{1}$, A. J. Clarke $^{2}$

${ }^{1}$ University of Southampton, Southampton, United Kingdom, ${ }^{2}$ Cardiff University, Cardiff, United Kingdom
Introduction: Some patients appear to 'know' whether they wish to have a genetic test or not, whilst others deliberate extensively before deciding. Little is understood about how patients deliberate such decisions. Previous research has focused on the result of the deliberation process, but little attention has been paid to the process of arriving at a decision itself and the role medical and lifeworld frames play in this deliberation. Our research explored both the deliberation and eventual decision made by patients at risk of Huntington's disease.

Methods: We recruited 15 patients who were considering predictive testing for Huntington's disease from four UK regional genetics services. We gathered qualitative data from patients' clinical consultations and reflective diaries to explore deliberation and patient interviews to explore decisions. We took an ethnographic approach to consultations, applied discourse analysis to clinic consultations and reflective diaries, and account analysis to the final interview with participants.

Results: Our findings show how consultations, discussions with others, misconceptions, and information seeking influence four key areas: 1. Patient knowledge of the options available; 2. Forecasting of emotions regarding different options; 3. Imagined futures and 4. Hypothetical scenarios (if/then). We compared clinical consultations with reflective diaries to further explore deliberation inside and outside the clinical appointment.

Conclusion: Our findings illustrate how medical frames sit alongside patient lifeworld frames and how this influences deliberation and shared decision making. We make recommendations regarding the development of decision support tools for use in clinical practice. ESRC Grant ES/R003092/1

L. M. Ballard: None. S. Doheny: None. A. M. Lucassen: None. A. J. Clarke: None.

\section{C23.4}

Negotiating autonomy and interdependence with family: how young people and health professionals navigate genetic testing for Li-Fraumeni syndrome

\section{R. Forbes Shepherd ${ }^{1,2 *}$, L. A. Keogh ${ }^{3}$, A. V. Werner-Lin ${ }^{4}$, M. B. Delatycki ${ }^{5,6}$, L. E. Forrest ${ }^{1,2}$}

${ }^{1}$ Parkville Familial Cancer Centre, Peter MacCallum Cancer Centre, Melbourne, Australia, ${ }^{2}$ Sir Peter MacCallum Department of Oncology, The University of Melbourne, Melbourne, Australia, ${ }^{3}$ Melbourne School of Population and Global Health, The University of Melbourne, Melbourne, Australia, ${ }^{4}$ School of Social Policy and Practice, University of Pennsylvania, Philadelphia, PA, United States, ${ }^{5}$ Victorian Clinical Genetics Service, Melbourne, 
Australia, ${ }^{6}$ Department of Paediatrics, The University of Melbourne, Melbourne, Australia

Background/Purpose: The intersection of adolescence and young adulthood (AYA: aged 15-39 years) with high multi-organ cancer risk caused by Li-Fraumeni syndrome (LFS), complicates the process and implications of genetic testing. This research aims to investigate how AYAs experience, and health professionals negotiate, this process.

Methods: A multi-method approach using qualitative interviews with AYAs together with a survey of Australasian genetic and oncology health professionals examined experiences and practice of LFS genetic testing.

Findings: Thirty AYAs (mean age 25.5; 26 TP53+ve; 4 at $50 \%$ risk) participated. Reliance on family at this life stage complicated AYAs' autonomous decision-making capacity for genetic testing, with parent-driven testing being common among adolescents. Adolescents who underwent diagnostic testing had limited involvement in genetic testing decisions due to a power differential between parents and providers. Forty-three respondents completed the survey (56\% genetic counsellors), $65 \%$ had facilitated predictive TP53 testing for AYAs, 33\% had facilitated testing for minors ( $<18$ years). Using vignettes, respondents were more supportive of testing for emotionally mature compared to immature minors $(\mathrm{p}=0.009)$, and more supportive of adolescent wishes compared to parental wishes for testing $(\mathrm{p}=0.020)$ when families held discordant views on testing.

Conclusion: Despite health professionals supporting AYA autonomy in vignettes, the reality of genetic testing for LFS described by AYAs was more complex and subtle, involving both developmental and familial considerations, which require developmentally appropriate genetic counselling.

R. Forbes Shepherd: None. L. A. Keogh: None. A. V. Werner-Lin: None. M. B. Delatycki: None. L. E. Forrest: None.

\section{C23.5}

\section{Exploring outcomes from the Deciphering Developmen-} tal Disorders study

\author{
H. Copeland ${ }^{1,2 *}$, E. Kivuva ${ }^{1}$, G. Hall ${ }^{2}$, T. DDD Study ${ }^{3}$, C. \\ Wright $^{4}$
}

${ }^{I}$ Department of Clinical Genetics, RD\&E, Exeter, United Kingdom, ${ }^{2}$ School of Biological Sciences, University of Manchester, Manchester, United Kingdom, ${ }^{3}$ Wellcome Trust Sanger Institute, Wellcome Genome Campus, Cambridge, United Kingdom, ${ }^{4}$ Institute of Biomedical and Clinical Science, University of Exeter Medical School, Exeter, United Kingdom
The Deciphering Developmental Disorders (DDD) Study is investigating the genetic aetiology of abnormal development in $>13,500$ children with severe developmental disorders from across the UK and Ireland. Diagnoses have been identified in around $35 \%$ of individuals, and over 50 genes have been newly implicated in developmental disorders. We sought to investigate the clinical and psychosocial outcomes of receiving a genetic diagnosis in a subset of DDD participants. Individuals recruited through the Royal Devon and Exeter NHS Trust who received a confirmed genetic diagnosis from the DDD study before August 2019 were selected for inclusion $(n=112)$. Genetics notes were reviewed to assess pre-defined clinical outcomes. Disease-specific treatment was indicated for seven individuals, including Carbamazepine for seizure control in individuals with pathogenic variants in $K C N Q 2$, and antenatal L-serine treatment for pregnancies at risk of pathogenic variants in $P H G D H$. Additionally, 69 individuals were referred to other specialties for investigations or ongoing screening, 25 individuals were recruited to further genetic research studies, three families were able to access prenatal testing techniques, and 87 families were given or signposted to disease-specific information and/or genespecific patient support groups. Individuals $(n=16)$ who had no recorded clinical outcomes were invited to interview to assess the psychosocial impact of the diagnosis for the individual and wider family. Preliminary results highlight the broad impact of a diagnosis, including clinical outcomes for both the patient and family, as well as wider social and psychological benefits. This presentation will discuss the clinical and psychosocial impact of receiving a genetic diagnosis through DDD.

H. Copeland: None. E. Kivuva: None. G. Hall: None. T. DDD Study: None. C. Wright: None.

\section{C23.6}

Improving the communication of genomics results to patients and families with rare diseases using Experience-Based Co-Design (EBCD)

\section{A. Costa ${ }^{1,2}$, V. Frankova ${ }^{3,4 *}$, E. Alexander ${ }^{5}, A$. Arellanesová $^{6}$, V. Bros-Facer ${ }^{7}, J$. Clayton-Smith ${ }^{5,8}$, G.

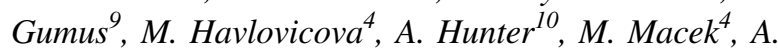 Metcalfe $^{11}$, C. Patch ${ }^{12,2}$, R. Pourová-Kremlíková ${ }^{4}, M$. Pritchard $^{13}$, G. Robert ${ }^{1}$, L. Roberts ${ }^{13}$}

${ }^{1}$ Florence Nightingale Faculty of Nursing, Midwifery \& Palliative Care King's College, London, United Kingdom, ${ }^{2}$ Society and Ethics Research, Connecting Science, Wellcome Genome Campus, Hinxton, United Kingdom, ${ }^{3}$ Institute for Medical Humanities, Charles University, First Faculty of Medicine, Prague, Czech Republic, ${ }^{4}$ Department of Biology and Medical Genetics, Charles University, 2nd 
Faculty of Medicine and University Hospital Motol, Prague, Czech Republic, ${ }^{5}$ Manchester Centre For Genomic Medicine, University of Manchester, St Mary's Hospital, Manchester, United Kingdom, ${ }^{6} \mathrm{CAVO}$, Prague, Czech Republic, ${ }^{7}$ EURORDIS, Paris, France, ${ }^{8}$ Division of Evolution and Genomic Sciences School of Biological Sciences University of Manchester, Manchester, United Kingdom, ${ }^{9}$ EURORDIS, Barcelona, Spain, ${ }^{10}$ Genetic Alliance UK, London, United Kingdom, ${ }^{11}$ Sheffield Hallam University, Sheffield, United Kingdom, ${ }^{12}$ Genomics England, Queen Mary University of London, London, United Kingdom, ${ }^{13}$ SWAN UK, London, United Kingdom

Introduction: We present the methods and findings of a research study aimed to improve the communication of genomic results to families with rare diseases. The presentation will include a short video produced with family participants.

Materials and methods: The study was set in two genetic services, one in the United Kingdom and one in the Czech Republic. Using EBCD, we carried out observations of clinical appointments and interviews with families $(n=18)$ and health professionals $(n=22)$. Over a series of workshops, we worked with participants to identify priorities for improvement and develop more appropriate ways of communicating genomic results.

Results: Priorities for improvement identified included: i) communication at the point testing (UK); ii) counselling on feeling of guilt (CZ) and iii) follow up care (both sites). To address these, participants co-designed two types of interventions: i) educational interventions for workforce and ii) service model improvements.

Conclusions: The results provide important evidence on key competencies for the workforce in the new genomic era, and practical recommendations to achieve effective family-centred communication. They also indicate the need for new models of service delivery and greater attention to follow up care after results have been shared. Finally, the study offers practical lessons on how to enable patient and family involvement to co-designing genomic services that can best meet their needs.

The study is part of the larger Solve-RD project. The Solve RD has received funding from the European Union's Horizon 2020 research and innovation programme under grant agreement No 779257.

A. Costa: None. V. Frankova: None. E. Alexander: None. A. Arellanesová: None. V. Bros-Facer: None. J. ClaytonSmith: None. G. Gumus: None. M. Havlovicova: None. A. Hunter: None. M. Macek: None. A. Metcalfe: None. C. Patch: None. R. Pourová-Kremlíková: None. M. Pritchard: None. G. Robert: None. L. Roberts: None.

\section{C24 Best Posters 2}

\section{C24.02}

A novel disorder involving dyshematopoiesis, inflammation and HLH due to aberrant CDC42 function

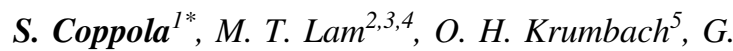
Prencipe $^{6}$, I. Insalaco ${ }^{6}$, C. Cifaldi ${ }^{7,8}$, I. Brigida ${ }^{9}$, E. Zara $^{1}$, S. Scala ${ }^{9}$, S. Martinelli ${ }^{10}$, M. Di Rocco ${ }^{10,11}$, A. Pascarella ${ }^{6}$,

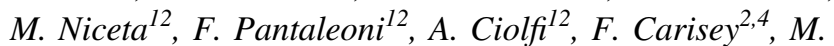
Akbarzadeh $^{5}$, L. Farina ${ }^{1,6}$, L. Pannone ${ }^{10,12}$, H. C. Erichsen $^{13}$, A. C. Horne ${ }^{14,15}$, Y. Bryceson ${ }^{16,17}$, L. TorralbaRaga $^{16}$, K. Ramme ${ }^{18,8}$, A. Finocchi ${ }^{19,8}$, F. Locatelli ${ }^{20}, C$. Cancrini $^{19,8}$, A. Aiuti $^{9}$, M. R. Ahmadian ${ }^{5}$, J. S. Orange ${ }^{2}, F$. De Benedetti ${ }^{6}$, M. Tartaglia ${ }^{12}$

${ }^{l}$ National center for rare diseases, Istituto Superiore di Sanità, Rome, Italy, ${ }^{2}$ Department of Pediatrics, Columbia University, Irving Medical Center, New York, NY, United States, ${ }^{3}$ Translational Biology and Molecular Medicine Graduate Program and Medical ScientistTraining Program, Baylor College of Medicine, Houston, TX, United States, ${ }^{4}$ Department of Pediatrics, Baylor College of Medicine, Houston, TX, United States, ${ }^{5}$ Institute of Biochemistry and Molecular Biology II, Medical Faculty of the Heinrich-Heine University, Düsseldorf, Germany, ${ }^{6}$ Division of Rheumatology, Ospedale Pediatrico Bambino Gesù, IRCCS, Rome, Italy, ${ }^{7}$ Department of Pediatrics, Ospedale Pediatricon Bambino Gesù, IRCCS, Rome, Italy, ${ }^{8}$ Department of Systems Medicine, University of Rome Tor Vergata, Rome, Italy, ${ }^{9}$ San Raffaele Telethon Institute for Gene Therapy (SR-TIGET), IRCCS San Raffaele Scientific Institute, Milan, Italy, ${ }^{10}$ Department of Oncology and Molecular Medicine, Istituto Superiore di Sanità, Rome, Italy, ${ }^{11}$ Department of Cellular Biotechnology and Haematology, Sapienza University of Rome, Rome, Italy, ${ }^{12}$ Genetics and Rare Diseases Research Division, Ospedale Pediatrico Bambino Gesù, IRCCS, Rome, Italy, ${ }^{13}$ Department of Pediatric and Adolescent Medicine, Oslo University Hospital, Oslo, Norway, ${ }^{14}$ Pediatric Rheumatology, Astrid Lindgren Childrens Hospital, Karolinska University Hospital, Stockholm, Sweden, ${ }^{15}$ Department of Women's and Children's Health, Karolinska Institutet, Karolinska University Hospital Solna, Stockholm, Sweden, ${ }^{16}$ Department of Medicine, Karolinska Institutet, Karolinska University Hospital Huddinge, Stockholm, Sweden, ${ }^{17}$ Broegelmann Research Laboratory, Institute of Clinical Sciences, University of Bergen, Bergen, Norway, ${ }^{18}$ Pediatric Hematology, Immunology and HCT Section, Astrid Lindgren Children's Hospital, Karolinska University Hospital, Stockholm, Sweden, ${ }^{19}$ Department of Pediatrics, Ospedale PediatricoBambino Gesù, IRCCS, Rome, Italy, 
${ }^{20}$ Department of Pediatric Hematology and Oncology, Ospedale Pediatrico Bambino Gesù, IRCCS, Rome, Italy

Introduction: The diagnosis and delineation of novel genetic syndromes is often difficult given the limited availability of patients, genetic heterogeneity, and clinical variability. Hemophagocytic lymphohistiocytosis (HLH) is characterized by hyper-inflammation driven by excessive activation and expansion of macrophages and defective Natural Killer (NK) cytotoxicity; it can be fatal if untreated. Here, we identify a previously unrecognized hematological and autoinflammatory condition in four unrelated patients with superimposable features, including neonatal-onset trilinear cytopenia with dyshematopoiesis, autoinflammation, rash, and HLH.

Methods: To delineate this new syndrome, we performed phenotype and whole-genome/exome analyses. In silico, in vitro and in vivo (C. elegans) assays allowed to characterize the impact of mutation on localization and function.

Results: All patients shared the same de novo CDC42 mutation (Chr1:22417990C $>$ T, p.R186C) and altered hematopoietic compartment, immune dysregulation, and inflammation. We documented unique effects of the p. $\mathrm{R} 186 \mathrm{C}$ on CDC42 localization and function. Indeed, $\mathrm{CDC} 42^{\wedge} \mathrm{R} 186 \mathrm{C}$ showed impaired interaction with known CDC42 regulators and effectors, including RHOGDI, IQGAP1 and WASP, leading to aberrant Golgi accumulation, cytoskeleton rearrangement and reduced growth and migration, which likely HB1underlie the distinctiveness of the trait of this syndrome. Aberrant CDC42 function caused defective NK cytotoxicity, which likely contributes to the HLH phenotype. Timely therapeutic neutralization of IL-1? and IFN? was critical to the survival of one patient, who underwent successful bone marrow transplantation.

Conclusions: The presented data emphasize the crucial role played by $\mathrm{CDC} 42$ in hematopoiesis and inflammation. Early recognition of the disorder and establishment of treatment followed by bone marrow transplant are important to survival.

S. Coppola: None. M. T. Lam: None. O. H. Krumbach: None. G. Prencipe: None. I. Insalaco: None. C. Cifaldi: None. I. Brigida: None. E. Zara: None. S. Scala: None. S. Martinelli: None. M. Di Rocco: None. A. Pascarella: None. M. Niceta: None. F. Pantaleoni: None. A. Ciolfi: None. F. Carisey: None. M. Akbarzadeh: None. L. Farina: None. L. Pannone: None. H. C. Erichsen: None. A. C. Horne: None. Y. Bryceson: None. L. Torralba-Raga: None. K. Ramme: None. A. Finocchi: None. F. Locatelli: None. C. Cancrini: None. A. Aiuti: None. M. R. Ahmadian: None. J. S. Orange: None. F. De Benedetti: None. M. Tartaglia: None.

\section{C24.04}

Loss of costameric supervillin causes myopathy with myofibrillar disorganization and autophagic vacuoles

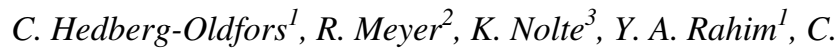
Lindberg $^{4}$, K. Karason ${ }^{5}$, I. J. Thuestad ${ }^{6}, K$. Visuttijai ${ }^{l}, M$.

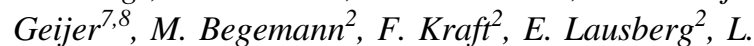
Hitpaß ${ }^{9}$, R. Götzl ${ }^{10}$, E. J. Luna ${ }^{11}$, H. Lochmüller ${ }^{12,13}, S$.

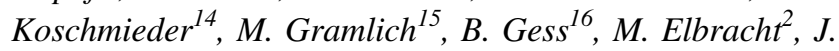
Weis $^{3}$, I. Kurth ${ }^{2}$, A. Oldfors ${ }^{1}$, C. Knopp ${ }^{2 *}$

${ }^{I}$ Department of Pathology and Genetics, Sahlgrenska Academy, University of Gothenburg, Gothenburg, Sweden, ${ }^{2}$ Institute of Human Genetics, Medical Faculty, RWTH Aachen University, Aachen, Germany, ${ }^{3}$ Institute of Neuropathology, Medical Faculty, RWTH Aachen University, Aachen, Germany, ${ }^{4}$ Department of Neurology, Neuromuscular Centre, Sahlgrenska University Hospital, Gothenburg, Sweden, ${ }^{5}$ Department of Cardiology and Transplant Institute, Sahlgrenska University Hospital, Gothenburg, Sweden, ${ }^{6}$ Department of Pediatrics, Skane University Hospital, Malmö, Sweden, ${ }^{7}$ Department of Radiology, Sahlgrenska Academy, University of Gothenburg, Gothenburg, Sweden, ${ }^{8}$ Department of Clinical Sciences, Lund University, Lund, Sweden, ${ }^{9}$ Institute of Human Genetics, MeDepartment of Diagnostic and Interventional Radiology, Medical Faculty, RWTH Aachen Universitydical Faculty, RWTH Aachen University, Aachen, Germany, ${ }^{10}$ Department of Plastic Surgery, Hand and Burn Surgery, Medical Faculty, RWTH Aachen University, Aachen, Germany, ${ }^{11}$ Division of Cell Biology and Imaging, Department of Radiology, University of Massachusetts Medical School, Worcester, MA, United States, ${ }^{12}$ Children's Hospital of Eastern Ontario Research Institute, Division of Neurology, Department of Medicine, The Ottawa Hospital, Ottawa, ON, Canada, ${ }^{13}$ Brain and Mind Research Institute, Ottawa, ON, Canada, ${ }^{14}$ Department of Hematology, Onkology, Hemostaseology and Stem Cell Transplantation, Medical Faculty, RWTH Aachen University, Aachen, Germany, ${ }^{15}$ Department of Invasive Electrophysiology, Medical Faculty, RWTH Aachen University, Aachen, Germany, ${ }^{16}$ Department of Neurology, Medical Faculty, RWTH Aachen University, Aachen, Germany

Introduction: Archvillin, the muscle specific isoform of supervillin encoded by the SVIL gene, is a large sarcolemmal protein. Despite an important role of supervillin in muscle cell physiology has been suggested, no muscular disease associated with SVIL mutations has been described. We here report a new structural myopathy in four individuals of two unrelated, consanguineous families caused by biallelic loss-of-function mutations in SVIL. 
Material and Methods: Patients were comprehensively investigated clinically, and whole exome sequencing on genomic DNA was performed in all affected individuals. Skeletal muscle biopsies were morphological analyzed by enzyme and immunohistochemical techniques and electron microscopy.

Results: Affected patients share a distinctive phenotype that includes a wide neck, anteverted shoulders, and a prominent trapezius muscle together with variable contractures besides further skeletal and mild heart muscle involvement. Muscle biopsies of both families demonstrated complete loss of supervillin in muscle tissue by western blot and immunohistochemical analyses. Comprehensive light and electron microscopical investigations of muscle biopsies showed striking similarities between patients and revealed a structural myopathy with prominent lobulated type 1 fibers, myofibrillar disintegration and signs of altered proteostasis and impaired autophagy.

Conclusions: The results underline the importance of supervillin for the structural integrity of muscle fibers in humans and show that recessive loss-of-function mutations in SVIL cause a novel myopathy with distinct histological, ultrastructural and clinical findings.

Funding: This study was supported by the Swedish Research Council to AO (grant number 2018-02821) and by the German Research Foundation (DFG) to IK (KU1587/4-1).

C. Hedberg-Oldfors: None. R. Meyer: None. K. Nolte: None. Y. A. Rahim: None. C. Lindberg: None. K. Karason: None. I. J. Thuestad: None. K. Visuttijai: None. M. Geijer: None. M. Begemann: None. F. Kraft: None. E. Lausberg: None. L. Hitpaß: None. R. Götzl: None. E. J. Luna: None. H. Lochmüller: None. S. Koschmieder: None. M. Gramlich: None. B. Gess: None. M. Elbracht: None. J. Weis: None. I. Kurth: None. A. Oldfors: None. C. Knopp: None.

\section{C24.06}

Genome wide methylation profiling, EpiSign, as a diagnostic test

\section{Alders ${ }^{1 *}$, P. Henneman ${ }^{l}$, E. Aref-Eshghi ${ }^{2}$, A. Venema ${ }^{l}$, J. Kerkhof ${ }^{2}$ B. Sadikovic ${ }^{2,3}$, M. Mannens ${ }^{1}$}

${ }^{1}$ Amsterdam UMC, Amsterdam, Netherlands, ${ }^{2}$ London Health Sciences Centre, London, ON, Canada, ${ }^{3}$ Western University, London, ON, Canada

A growing number of neurodevelopmental syndromes are shown to have unique genomic DNA methylation profiles. These episignatures can be used to diagnose patients with such syndromes. We have recently implemented genome wide methylation profiling as a diagnostic test in our laboratory. This test, named EpiSign(v1), is able to recognize 22 syndromes, 14 of which are syndromes with specific episignatures, (for example for Kabuki syndrome, BAFopathies and Cornelia de Lange syndrome), 6 are imprinting disorders and 2 are repeat expansion disorders. Validation of the test was based on 38 positive samples (with known pathogenic variant) and 24 negative controls. All samples were called correctly provided that the DNA was extracted from peripheral blood and there was no low mosaicism for the pathogenic variant. Reasons for requesting an EpiSign clinical test were: the presence of a variant of unknown clinical significance, clinical diagnosis without molecular confirmation, or as a test complementary to WES/WGS, without a prior (suspected) diagnosis. In approximately $40 \%$ of cases with a suspected (VUS present) or clinical diagnosis, EpiSign was able to confirm this diagnosis. This shows that EpiSign is a powerful new diagnostic tool, in particular for patients for whom conventional genetic testing was inconclusive. In March 2020 an updated version of EpiSign will be available (EpiSignv2), allowing diagnosis of an additional 23 syndromes (45 in total). For a current list of syndromes detected by EpiSign see https://genomedia gnostics.amsterdamumc.nl/

M. Alders: None. P. Henneman: None. E. Aref-Eshghi: None. A. Venema: None. J. Kerkhof: None. B. Sadikovic: None. M. Mannens: None.

\section{C24.08}

The landscape of BRCA1 and BRCA2 variants from 300,000 individuals in the UK Biobank population sampling

\section{Z. Ghazoui*}

\section{AstraZeneca, Cambridge, United Kingdom}

Prior prevalence estimates of BRCA1 and BRCA2 proteintruncating variants (PTVs) have been generally limited to studies that have ascertained for cancer patients or individuals with a family history of cancer. The aim of this study is to utilise one of the largest sequenced Biobank populations to investigate the prevalence of BRCA1 and BRCA2 PTVs and ClinVar pathogenic/likely pathogenic classified missense variants. We analysed whole-exome sequence data from 300,000 UK Biobank participants (https://www. ukbiobank.ac.uk/), which is a prospective cohort study linked to deep phenotypic data. Among the 300,000 exomes, we identified 1332 carriers of high confidence rare PTVs in BRCA1/2 and another set of 62 carriers of rare ClinVar pathogenic/likely pathogenic classified missense variants in BRCA1/2. Of the 1394 BRCA1/2 putatively pathogenic variant carriers, $529(38.0 \%)$ have been diagnosed with cancer, $455(32.6 \%)$ have a reported family history of cancer but are not diagnosed with cancer and 410 (29.4\%) have no reported history (or family history) of cancer. For the 
529 individuals with a cancer diagnosis we further evaluated the contribution that they have to 100 distinct ICD10 primary cancer phenotypes and identified that ovarian $(5.3 \%$ of reported), breast ( $2 \%$ of reported), prostate ( $1 \%$ of reported), pancreas (1.5\% of reported) and lung (1.1\% of reported) cancers are amongst the cancers that are significantly enriched for these BRCA1/2 putatively pathogenic variants. This study provides an unbiased summary of germline BRCA1/2 contribution to various primary cancers and highlights the value of careful variant interpretation.

Z. Ghazoui: A. Employment (full or part-time); Significant; AstraZeneca.

\section{C24.09}

Nanopore-sequencing-based high-resolution karyotyping of AML patients

\author{
F. Kraft ${ }^{*}$, P. Krüger ${ }^{1}$, S. Koschmieder ${ }^{2}$, E. Jost ${ }^{2}$, M. \\ Crysandt ${ }^{2}$, I. Kurth ${ }^{1}$
}

${ }^{1}$ Institute of Human Genetics, University Hospital RWTH Aachen, Aachen, Germany, ${ }^{2}$ Department of Medicine (Hematology Oncology, Hemostaseology, and Stem Cell Transplantation), University Hospital RWTH Aachen, Aachen, Germany

Introduction: The current diagnostic scheme for classification of acute myeloid leukemia (AML) patients is usually based on cytogenetic and molecular biology methods. The turnaround time for karyotype analysis is up to two weeks, due to the indispensable cultivation of hematopoietic cells. Although initial PCR or FISH-based testing for recurrent structural variations (SV) can be carried out within few hours, the AML classification is demanding and requires accurate karyotyping.

Methods: We utilized nanopore-sequencing to overcome the time-consuming karyotyping and further increase resolution and accelerate SV analysis for AML classification. In detail, low-coverage whole genome sequencing (LC-WGS) was carried out in combination with a modified SMURFseq protocol on AML bone marrow or blood samples (?20\% blasts). LC-WGS data was used to identify classification-relevant SV in AML. Genome-wide CNV analysis was done from SMURFSeq data.

Results: For all 16 samples the results from nanoporesequencing confirmed the SV identified by conventional cytogenetic and FISH analyses. Translocations, inversions, tandem duplications and CNVs were identified at single base resolution. We could detect additional small CNVs in growth regulating genes which may modify the aggressiveness of the leukemia. Moreover, the procedure from DNA isolation to obtaining results requires less than $48 \mathrm{~h}$ and is not operator dependent. The method can also generate karyotype data from samples for which the cell cultivation failed.

Conclusion: We show that nanopore-sequencing can speed-up and potentially improve the comprehensive analysis of SV in AML. Rapid classification according to ELN risk stratification will immediately impact on targeted and risk adapted treatment of AML patients.

F. Kraft: None. P. Krüger: None. S. Koschmieder: None. E. Jost: None. M. Crysandt: None. I. Kurth: None.

\section{C24.10}

Classification of ATM variants identified in Spanish patients with suspicion of hereditary cancer

L. Feliubadalo ${ }^{1,2,3^{*}}$, M. Santamariña Peña $a^{4,5,6}$, L. M. Porras $^{7,8}$, A. Moles-Fernández ${ }^{9}$, A. T. Sánchez ${ }^{10,11}$, A. Blanco $^{12,13,5}$,O. Diez ${ }^{9,14}$, S. Gutiérrez-Enríquez ${ }^{9}$, M. de la Hoya $^{15,16}$, A. Lopez-Novo ${ }^{17}$, A. Osorio ${ }^{18,5}$, M. Pineda $^{1,2,3}$, D. Rueda ${ }^{19,20}$, C. Ruiz-Ponte ${ }^{21,5}$, A. Vega $a^{12,13,5}$, I. J. Molina ${ }^{22}$, X. de la Cruz ${ }^{7,8,23}$, C. Lázaro $^{1,2,3}$

${ }^{1}$ Hereditary Cancer Program, Catalan Institute of Oncology, IDIBELL, Hospitalet de Llobregat, Spain, ${ }^{2}$ Program in Molecular Mechanisms and Experimental Therapy in Oncology (Oncobell), IDIBELL, Hospitalet de Llobregat, Spain, ${ }^{3}$ Centro de Investigación Biomédica en Red de Cáncer (CIBERONC), Madrid, Spain, ${ }^{4}$ Grupo de Medicina Xenómica. Instituto de Investigación Sanitaria de Santiago de Compostela, Santiago de Compostela, Spain, ${ }^{5}$ CIBERER, Madrid, Spain, ${ }^{6}$ Universidad de Santiago de Compostela, Santiago de Compostela, Spain, ${ }^{7}$ Research Unit in Clinical and Translational Bioinformatics, Vall d'Hebron Institute of Research (VHIR), Barcelona, Spain, ${ }^{8}$ Universitat Autònoma de Barcelona, Barcelona, Spain, ${ }^{9}$ Hereditary Cancer Genetics Group, Vall d'Hebron Institute of Oncology (VHIO), Barcelona, Spain, ${ }^{10}$ Hereditary Cancer Program, Catalan Institute of Oncology, Barcelona, Spain, "Aberystwyth University, Aberystwyth, United Kingdom, ${ }^{12}$ Fundación Pública Galega Medicina Xenómica-SERGAS, Santiago de Compostela, Spain, ${ }^{13}$ Instituto de Investigación Sanitaria de Santiago de Compostela (IDIS), Santiago de Compostela, Spain, ${ }^{14}$ Area of Clinical and Molecular Genetics, University Hospital of Vall d'Hebron, Barcelona, Spain, ${ }^{15}$ CIBER-ONC, Instituto de Salud Carlos III, Madrid, Spain, ${ }^{16}$ Molecular Oncology Laboratory, Hospital Clinico San Carlos, IdISSC (Instituto de Investigación Sanitaria del Hospital Clínico San Carlos), Madrid, Spain, ${ }^{17}$ Fundacion Publica Galega de Medicina Xenómica, Grupo de Medicina Xenomica-Universidade de Santiago de Compostela, Instituto de Investigacion Santiago de Compostela (E035), Santiago de Compostela, Spain, ${ }^{18}$ Human Genetics Group, Spanish National Cancer Research Centre (CNIO), Madrid, Spain, 
${ }^{19}$ Digestive Cancer Research Group, 12 de Octubre Research Institute, Madrid, Spain, ${ }^{20}$ Molecular Biology Laboratory, 12 de Octubre University Hospital, Madrid, Spain, ${ }^{21}$ Fundacion Publica Galega de Medicina Xenomica, Grupo de Medicina Xenomica-Universidade de Santiago de Compostela, Instituto de Investigacion Sanitaria de Santiago de Compostela (E035), Santiago de Compostela, Spain, ${ }^{22}$ Institute of Biopathology and Regenerative Medicine, University of Granada, Granada, Spain, ${ }^{23}$ Institució Catalana de Recerca i Estudis Avançats (ICREA), Barcelona, Spain

The use of NGS gene panels for clinical testing has increased the diagnostic yield, but also the frequency of variants of unknown significance (VUS) per patient. With the aim of improving and standardizing variant classification, we'll create a national registry of genetic variants in cancer predisposing-genes. To that end, seven Spanish molecular laboratory groups currently using NGS panels for hereditary cancer diagnostics created a variant database. Our current database gathers all variants with less than $1 \%$ allele frequency in all GnomAD outbred populations, starting with the ATM gene, study because of its moderate increase in breast cancer risk and its remarkable number of VUSes. The methodology consisted of: 1) selection and curation of 50 representative variants from our cohort; 2) evaluation of each by three members; and 3) discussion of evidences for classification in monthly teleconferences. As ATM-specific guidelines have not been published, we have previously carried out our own ACMG/AMP criterion adjustment. So far, 842 variants belonging to 730 tested patients with different hereditary cancer conditions. Among the 265 unique variants, 97 appeared more than once, and 35 had differences in classification among groups. Our new ATM-specific criteria have allowed us to improve and standardize the classification of the 50 pilot variants. We'll next submit our classifications to international databases and extend our database to other Spanish participants and hereditary cancer genes. Grant support: Carlos III National Health Institute - FEDER funds [PI19/00553; PI16/00563; PI16/01898; SAF2015-68016-R and CIBERONC]; the Government of Catalonia [PERIS_MedPerCan and URDCat projects, 2017SGR1282 and 2017SGR496].

L. Feliubadaló: None. M. Santamariña Peña: None. L. M. Porras: None. A. Moles-Fernández: None. A. T. Sánchez: None. A. Blanco: None. O. Diez: None. S. GutiérrezEnríquez: None. M. de la Hoya: None. A. Lopez-Novo: None. A. Osorio: None. M. Pineda: None. D. Rueda: None. C. Ruiz-Ponte: None. A. Vega: None. I. J. Molina: None. X. de la Cruz: None.

\section{C24.12}

Reduced male reproductive success drives selective constraint on human genes

E. J. Gardner ${ }^{*}$, M. D. C. Neville ${ }^{l}$, K. E. Samocha ${ }^{l}$, M. E. K. Niemi ${ }^{l}$, K. J. Barclay ${ }^{2}$, M. Kolk ${ }^{3}$, G. Kirov ${ }^{4}$, H. C. Martin $^{l}$, M. E. Hurles ${ }^{l}$

${ }^{1}$ Wellcome Sanger Institute, Hinxton, United Kingdom, ${ }^{2}$ Max Planck Institute for Demographic Research, Rostock, Germany, ${ }^{3}$ Stockholm University, Stockholm, Sweden, ${ }^{4}$ School of Medicine, Cardiff University, Cardiff, United Kingdom

Sequencing of human populations has revealed highly variable patterns of natural selection acting on human genes, with a minority of genes appearing to be under strong selection against heterozygous variants that abolish the function of the encoded protein. Although these selectively constrained genes are strongly enriched for dominant single gene disorders, for most of these genes the nature of the selective constraint is unknown. We called large deletions from SNP genotyping data on 500K UK Biobank participants and analysed protein truncating variants from exome data on 50K UK Biobank participants. We determined the cumulative burden of likely loss-of-function variants in each UK Biobank participant, weighted according to the degree of selective constraint of the impacted gene(s), which we term their Shet burden. We found that increasing Shet burden results in substantially reduced male reproductive success, driven by an increased risk of childlessness $(p=4 \times 10-15)$. This reduction in reproductive success is much weaker in females $(p=$ 0.0026). Shet burden is also associated with reduced fluid intelligence, educational attainment and household income, but without strong sex-bias. We present evidence that this reduced male reproductive success is most likely mediated by cognitive and behavioural traits, which, due to female preferences for mating partners likely to invest resources in their offspring, renders male carriers of such variants less able to find mating partners. Our findings support a substantial role for sexual selection due to mate choice preferences shaping the recent evolution of human genes.

E. J. Gardner: None. M. D. C. Neville: None. K. E. Samocha: None. M. E. K. Niemi: None. K. J. Barclay: None. M. Kolk: None. G. Kirov: None. H. C. Martin: None. M. E. Hurles: E. Ownership Interest (stock, stock options, patent or other intellectual property); Modest; Congenica. 


\section{C24.13}

Widespread sexual differences in genetic architecture in UK Biobank

\section{E. Bernabeu ${ }^{I *}$, O. Canela-Xandri ${ }^{2}, K$. Rawlik $^{l}$, A. Talenti ${ }^{I}$, J. Prendergast ${ }^{l}$, A. Tenesa ${ }^{l, 2}$}

${ }^{I}$ The Roslin Institute, University of Edinburgh, Edinburgh, United Kingdom, ${ }^{2}$ MRC Human Genetics Unit, University of Edinburgh, Edinburgh, United Kingdom

Despite males and females sharing nearly identical genomes, there are differences between the sexes in complex traits and in the risk, incidence, and prevalence of a wide array of diseases. Gene by sex interactions (GxS) are thought to underlie some of this sexual dimorphism. However, the extent and basis of these interactions are poorly understood. Here we provide insights into both the scope and mechanism of GxS across the genome of circa 450,000 individuals of European ancestry and 530 complex traits in UK Biobank. We report sex differences in heritability for 71 traits, through genetic correlations for 69 traits, and in associations of genetic variants located in the autosome and X-chromosome for 103 traits. We also show that sex specific polygenic risk scores may lead to a modest improvement of phenotypic prediction and highlight how sex agnostic analyses could be missing loci of interest. Finally, we studied the potential functional role of this dimorphism through sex-biased eQTL and gene-level analyses. This study marks the largest examination of the genetics of sexual dimorphism to date. Our findings parallel previous reports, proving the widespread presence of sexual genetic heterogeneity across complex traits, though be it of generally modest magnitude. This warrants the need for future studies to consider sex-stratifying analyses, in order to both shed light into possible sex-specific molecular mechanisms as well as improve prediction of high-level phenotypes.

E. Bernabeu: None. O. Canela-Xandri: None. K. Rawlik: None. A. Talenti: None. J. Prendergast: None. A. Tenesa: None.

\section{C24.14}

Profiling the chromatin landscape of early neurodevelopment using scATAC-seq

\section{C. Mannens ${ }^{*}$, L. Hu, E. P. Braun, P. Lönnerberg, S. Linnarsson}

\section{Karolinska institutet, Solna, Sweden}

Understanding the molecular processes underlying healthy development of the human brain is crucial in the modelling and investigation of neurodevelopmental disorders. While many evolutionarily conserved developmental processes are well studied in mouse models, our understanding of humanspecific neurodevelopmental processes is limited. Our lab aims to provide transcriptomic and epigenomic reference atlases with single-cell resolution. In this ongoing project, we are characterizing chromatin accessibility of cell types in the human brain during early development (5 - 13 p.c.w.) using single-cell ATAC-sequencing. So far, we have sequenced a few tens of thousands of cells and will profile up to one million individual human cells, identifying both region- and cell lineage-specific enhancers as well as enriched motifs and gene accessibility. We use a two-step approach to feature identification, first performing superficial decomposition and clustering on binned genomic ranges to stratify peak calling and then performing Hierarchical Poisson Factorization on more detailed PeakxCell data to identify lineages and cell types. We have identified 29 clusters and 325.484 unique peaks. 115.048 of these peaks are intergenic and 28.622 of these peaks (25\%) don't align to any known element in the GENCODE GRCh38 reference, indicating the presence of unannotated cell lineage specific enhancers. To validate the cluster identities and lineages, our scATAC-seq data will be integrated with the scRNA-seq data that has been acquired as part of the Human Developmental Cell Atlas initiative. This combined resource may serve as a reference for future studies relating to development of the human nervous system. European Union - H2020-SC1-BHC-2018-2020 - BRAINTIME

C. C. Mannens: None. L. Hu: None. E. P. Braun: None. P. Lönnerberg: None. S. Linnarsson: None.

\section{C25 Late Breaking Session}

\section{C25.1}

\section{Deciphering genomic inversions}

C. M. Grochowski ${ }^{*}$, M. Pettersson ${ }^{2}, J$. Eisfeldt ${ }^{2}, M$. Gandhi $^{1,3}$, D. Pehlivan ${ }^{l}$, C. Gonzaga-Jauregui ${ }^{4}, M$. Withers $^{1}$, P. Stankiewicz, ${ }^{1,5}$, A. C. V. Krepischi ${ }^{6}$, J. R. Lupski $^{l}$, A. Lindstrand ${ }^{2}$, C. M. B. Carvalho ${ }^{1}$

${ }^{1}$ Baylor College of Medicine, Houston, TX, United States, ${ }^{2}$ Karolinska Institutet, Stockholm, Sweden, ${ }^{3}$ University of Texas MD Anderson Cancer Center, Houston, TX, United States, ${ }^{4}$ Regeneron, Tarrytown, NY, United States, ${ }^{5}$ Baylor Genetics, Houston, TX, United States, ${ }^{6}$ University of São Paulo, SP, Brazil

Genomic inversions are a class of structural variation (SV) relevant in evolution, speciation and disease but challenging to detect/resolve. Copy number neutral inversions contain two breakpoints in cis, often located within repetitive 
regions. Contrastingly, inversions accompanied by copy number variation $(\mathrm{CNV}$, deletions/duplications/triplications) may have more than two breakpoints. Those features contribute to the high error rates that characterize inversion detection resulting in many events being overlooked.

We hypothesize that proper detection/investigation of inversions require a strategy utilizing multiple methodologies including cytogenetics and next-generation sequencing. We applied karyotyping, short-read WGS, linked-read WGS, droplet digital PCR, array CGH, long-read WGS (ONT) or optical mapping (OM) in two cohorts of inversion carriers: 1. paracentric or pericentric inversions $(\mathrm{N}=18)$ and 2. inversions accompanied with complex rearrangements $(\mathrm{N}=3)$ and one individual with multiple de novo inversions. For cohort 1 , we achieved nucleotide-resolution of inversion for 13/18 (72\%) samples studied by WGS providing mechanistic insights and challenging the dogma of how most inversions are formed. Remarkably, we observed that $\sim 17 \%$ of apparently neutral inversions are accompanied by CNVs. For cohort 2, we observed that ONT/OM resolved the complex inversion structure through the use of single molecules preserving the architecture, enabling identification of multiple junctions within repetitive segments missed by WGS.

No single technology solves all SVs. While short-read WGS can resolve a proportion of neutral inversions, those mediated by repeats or accompanied by $\mathrm{CNVs}$ remain challenging. Utilization of multiple technologies and visualization of unbroken DNA through OM/ONT sequencing facilitates detection/resolution of structures containing multiple breakpoint junctions.

C. M. Grochowski: None. M. Pettersson: None. J. Eisfeldt: None. M. Gandhi: None. D. Pehlivan: None. C. Gonzaga-Jauregui: A. Employment (full or part-time); Significant; Regeneron Genomics Center. M. Withers: None. P. Stankiewicz: None. A. C. V. Krepischi: None. J. R. Lupski: E. Ownership Interest (stock, stock options, patent or other intellectual property); Modest; 23 and Me. F. Consultant/Advisory Board; Modest; Regeneron Pharmaceuticals, Novartis. A. Lindstrand: None. C. M. B. Carvalho: None.

\section{C25.2}

Polymorphic inversions underlie the shared genetic susceptibility of obesity-related diseases

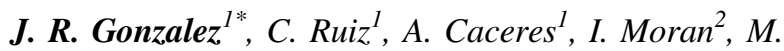
Lopez $^{I}$, L. Alonso ${ }^{2}$, I. Tolosana ${ }^{l}$, M. Guindo-Martinez ${ }^{3}, J$. M. Mercader ${ }^{3}$, T. Esko ${ }^{4}$, D. Torrents ${ }^{2}$, J. Gonzalez ${ }^{5}$, L. A. Perez-Jurado

${ }^{1}$ Barcelona Institute for Global Health, BARCELONA, Spain, ${ }^{2}$ Barcelona Supercomputing Center, BARCELONA,
Spain, ${ }^{3}$ Broad Institute, BOSTON, MA, United States, ${ }^{4}$ University of Tartu, BARCELONA, Estonia, ${ }^{5}$ Universitat Pompeu Fabra, BARCELONA, Spain

Background: The burden of several common diseases including obesity, diabetes, hypertension, asthma, and depression is increasing in most world populations. The mechanisms underlying the numerous epidemiological and genetic correlations among these disorders remain largely unknown. Genomic inversions can alter the function of genes by multiple mechanisms affecting several diseases. Its impact remained largely unknown because they were difficult to genotype in large cohorts.

Methods: We performed an inversion association analysis including 21 inversions and 25 obesity-related traits, on a total of 408,898 Europeans, and validated the results in 67,299 independent individuals. Inversion calling was performed using our scoreInvHap tool that uses exiting GWAS data.

Results: Seven inversions were associated with multiple diseases while inversions and inversions 8p23.1, 16p11.2 and $11 \mathrm{q} 13.2$ were strongly associated with the cooccurrence of obesity with other common diseases. Transcriptome analysis across numerous tissues revealed strong candidate genes of obesity-related traits. Analyses in human pancreatic islets indicated the potential mechanism of inversions in the susceptibility of diabetes by disrupting the cis-regulatory effect of SNPs from their target genes.

Conclusions: We report the largest association study of genomic inversions and human traits that represents a breakthrough for genomic association of comorbid disorders. Inversions have major genetic contribution into the joint susceptibility to common diseases. The results in obesity and diabetes reveal a mechanism in which cisregulatory SNPs are separated from their target genes by inversion breakpoints. Future studies in other diseases are now accessible to the research community thanks to our inversion genotyping tool.

J. R. Gonzalez: None. C. Ruiz: None. A. Caceres: None. I. Moran: None. M. Lopez: None. L. Alonso: None. I. Tolosana: None. M. Guindo-Martinez: None. J. M. Mercader: None. T. Esko: None. D. Torrents: None. J. Gonzalez: None. L. A. Perez-Jurado: None.

\section{C25.3}

Discovery of rare variants associated with blood pressure regulation through meta-analysis of 1.3 million individuals

\author{
E. V. Feofanova*, the Blood Pressure-International \\ Consortiumof Exome chip Studies (BP-ICE), CHARGE \\ consortium, CHD Exome+, Exome BP, GoT2D:T2Dgenes, \\ deCODE, the Million Veteran Program (MVP)
}


Human Genetics Center, The University of Texas Health Science Center at Houston, Houston, TX, United States

Elevated blood pressure (BP) is a major risk-factor for cardiovascular disease worldwide. Most prior genetic studies of BP explored Single Nucleotide Variants (SNVs) with minor allele frequency $[\mathrm{MAF}]>0.01$. The majority of identified BP-SNVs are non-coding and the corresponding causal genes remain unknown. We explored the role of rare variants $(\mathrm{MAF}<=0.01)$ on $\mathrm{BP}$ and elucidated candidate causal BP genes, to further our understanding of BP regulation.

The Exome array facilitates analyses of rare coding variants with potential functional consequences. Over $80 \%$ of SNVs on the array are rare and $\sim 80 \%$ are missense, implicating a candidate causal gene. We performed an Exome Array-Wide Association Study (EAWAS) of $\sim 247,000$ SNVs in $\sim 1.32$ million participants (95 studies) to identify SNVs associated with SBP, DBP, pulse pressure and hypertension. We additionally performed a rare-variant Genome-Wide Association Study (RV-GWAS) of 29 million imputed and genotyped SNVs in $\sim 670,000$ participants (UKBiobank, MVP).

We discovered 105 new BP-associated genomic regions, containing nine rare SNV-BP associations in the EAWAS and $23-$ in the RV-GWAS $\left(P<5 \times 10^{-8}\right)$; 55 additional rare variants were distinct novel SNVs within known BPassociated loci. Many BP-associated rare variants highlight potential candidate causal genes (e.g. GATA5, PLCB3), with $17 \%$ located in or near potentially druggable genes (e.g. NR3C2, NPPA).

Our study is the largest analysis of rare variants to date, revealing candidate genes at new and previously reported BP loci, highlighting potential therapeutic targets. We also report new observations from both rare and common loci enriching our understanding of genetic BP regulation in health and disease.

E. V. Feofanova: None.

\section{C25.4}

\section{Multi-Omics integration for molecular diagnostics of} mendelian disorders

R. Kopajtich ${ }^{1,2 *}$, D. Smirnov ${ }^{1,2}$, S. Loipfinger ${ }^{3}$, C. Meng ${ }^{4}$, D. Ghezzi ${ }^{5}$, K. Murayama ${ }^{6}$, J. A. Mayr ${ }^{7}$, P. Freisinger ${ }^{8}$, M. D. Metodiev ${ }^{9}$, A. Rötig ${ }^{9}$, T. Klopstock ${ }^{10}$, G. F. Hoffmann ${ }^{11}$, R. Santer ${ }^{12}$, F. Distelmaier ${ }^{13}$, R. Olsen ${ }^{14}$, D. PiekutowskaAbramczuk $^{15}$, P. Verloo ${ }^{16}$, C. Lamperti ${ }^{5}$, J. Gagneur ${ }^{3}$, C. Ludwig $^{4}$, H. Prokisch ${ }^{1,2}$

${ }^{1}$ Institute of Human Genetics, Technical University Munich, München, Germany, ${ }^{2}$ Institute of Neurogenomics, Helmholtz Center Munich, Munich, Germany, ${ }^{3}$ Department of
Informatics, Technical University Munich, Garching, Germany, ${ }^{4}$ Bavarian Center for Biomolecular Mass Spectrometry, BayBioMS, Technical University Munich, Freising, Germany, ${ }^{5}$ Unit of Molecular Neurogenetics, Istituto Neurologico 'Carlo Besta, Milan, Italy, ${ }^{6}$ Department of Metabolism, Chiba Children's Hospital, Chiba, Japan, ${ }^{7}$ Department of Paediatrics, Paracelsus Medical University, Salzburg, Austria, ${ }^{8}$ Department of Pediatrics, Klinikum Reutlingen, Reutlingen, Germany, ${ }^{9}$ INSERM U1163, Université Paris, Institut Imagine, Paris, France, ${ }^{10}$ Department of Neurology, Friedrich-Baur-Institute, Ludwig-Maximilians-University, Munich, Germany, ${ }^{11}$ Department of General Pediatrics, University Hospital Heidelberg, Heidelberg, Germany, ${ }^{12}$ University Medical Center Eppendorf, Hamburg, Germany, ${ }^{13} 13$ Department of General Pediatrics, Heinrich-Heine-University Düsseldorf, Düsseldorf, Germany, ${ }^{14}$ 14Research Unit for Molecular Medicine, Aarhus University and University Hospital, Aarhus N, Denmark, ${ }^{15}$ Department of Medical Genetics, The Children's Memorial Health Institute, Warsaw, Poland, ${ }^{16}$ Department of Internal Medicine and Paediatrics, Ghent University Hospital, Ghent, Belgium

With genomic advancement, came the shift from variant discovery to interpretation, which must be rigorous to avoid misinterpretation. Here, we analyzed 150 fibroblast cell lines from undiagnosed patients with suspected mitochondrial disorders. Transcriptome analysis facilitates genome-wide DNA variant interpretation on the RNA level, for both coding and non-coding variants. Proteomics allows quantification of proteins in one assay, removing the need for individual Western blot analyses. Missense variants accounting for over $50 \%$ of all mutations reported in HGMD. They may have no functional consequence or may exercise their pathogenicity by disrupting interactions or destabilizing the protein structure which can be detected by proteomics as aberrantly low expression. We established a protocol for quantitative deep proteome analysis allowing us to quantify a median of 7,500 proteins per sample. Discarding lowly expressed genes, around 13,000 transcripts are detectable. For the diagnosis of Mendelian disease, we analyzed three aberrant events: 1) aberrant splicing, 2) low expression, 3) monoallelic expression, and variants often present as a combination of these events. Combining DNA and RNA sequencing we increase the diagnostic yield by $15 \%$. Proteomics allowed us to validate 12/27 missense VUS (44\%). In additional 12 cases, we were able to reach a diagnosis by detecting low protein levels of known disease genes which have not been prioritized by WES. In 14 of those cases, we were able to validate the pathogenic character by the impact on additional proteins functionally associated. In summary, we established a multiomics integration pipeline and propose its application to advanced molecular diagnostics. 
R. Kopajtich: None. D. Smirnov: None. S. Loipfinger: None. C. Meng: None. D. Ghezzi: None. K. Murayama: None. J. A. Mayr: None. P. Freisinger: None. M. D. Metodiev: None. A. Rötig: None. T. Klopstock: None. G. F. Hoffmann: None. R. Santer: None. F. Distelmaier: None. R. Olsen: None. D. Piekutowska-Abramczuk: None. P. Verloo: None. C. Lamperti: None. J. Gagneur: None. C. Ludwig: None. H. Prokisch: None.

\section{C25.5}

Impaired complex I repair causes recessive Leber's hereditary optic neuropathy

S. L. Stenton ${ }^{1,2 *}$, N. Sheremet ${ }^{3}$, C. Catarino $^{4}$, N. Andreeva ${ }^{3}$,

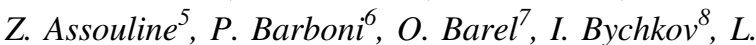
Caporali $^{9}$, P. Charbel Issa ${ }^{10}$, P. Freisinger ${ }^{11}$, S. Gerber ${ }^{12}$,

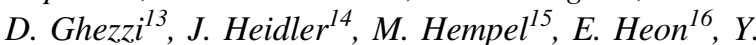
Itkis $^{17}$, E. Javasky ${ }^{7}$, J. Kaplan ${ }^{12}$, R. Kopajtich ${ }^{1,2}$, C. Kornblum $^{18}$, R. Kovacs-Nagy ${ }^{19,1}$, T. Krylova ${ }^{8}$, W. Kunz ${ }^{20}$, C. La Morgia ${ }^{9}$, C. Lamperti ${ }^{13}$, C. Ludwig ${ }^{21}$, P.

Malacarne $^{22}$, J. Mayr ${ }^{23}$, J. Meisterknecht $^{14}$, T. Nevinitsyna ${ }^{3}$, F. Palombo ${ }^{9}$, B. Pode-Shakked ${ }^{24}$, M. Shmelkova ${ }^{3}, M$. Tzadok $^{24}$, A. van der Ven ${ }^{15}$, C. Vignal-Clermont ${ }^{25}, M$. Wagner $^{1}$, E. Zakharova ${ }^{8}$, N. Zhorzholadze ${ }^{3}$, J. Rozet ${ }^{12}$, V. Carelli $^{9}$, P. Tsygankova ${ }^{8}$, T. Klopstock ${ }^{4}$, I. Wittig $^{14}, H$. Prokisch $^{1,2}$

${ }^{1}$ Institute of Human Genetics, Technische Universität München, München, Germany, ${ }^{2}$ Institute of Neurogenomics, Helmholtz Zentrum München, München, Germany, ${ }^{3}$ Federal State Budgetary Institution of Science "Research Institute of Eye Diseases", Moscow, Russian Federation, ${ }^{4}$ Department of Neurology, Friedrich-Baur-Institute, University Hospital of the Ludwig-Maximilians-Universität München, München, Germany, ${ }^{5}$ Fédération de Génétique et Institut Imagine, Université Paris Descartes, Hôpital Necker Enfants Malades, Paris, France, ${ }^{6}$ Scientific Institute San Raffaele, Milan, Italy, ${ }^{7}$ Genomics Unit, Sheba Cancer Research Center, Sheba Medical Center, Tel-Hashomer, Israel, ${ }^{8}$ Research Centre for Medical Genetics, Moscow, Russian Federation, ${ }^{9}$ IRCCS Istituto delle Scienze Neurologiche di Bologna, Bologna, Italy, ${ }^{10}$ Oxford Eye Hospital, Oxford University Hospitals NHS Foundation Trust, Oxford, United Kingdom, ${ }^{11}$ Department of Pediatrics, Klinikum am Steinenberg, Reutlingen, Germany, ${ }^{12}$ Laboratory genetics in ophthalmology (LGO), INSERM UMR1163 - Institute of genetic diseases, Imagine, Paris, France, ${ }^{13}$ Unit of Medical Genetics and Neurogenetics, Fondazione IRCCS Istituto Neurologico Carlo Besta, Milan, Italy, ${ }^{14}$ Functional Proteomics, SFB815 Core Unit, Medical School, Goethe University, Frankfurt am Main, Germany,

${ }^{15}$ Institute of Human Genetics, University Medical Center Hamburg-Eppendorf, Hamburg, Germany, ${ }^{16}$ The Hospital for Sick Children, Department of Ophthalmology and Vision Sciences, The University of Toronto, Toronto, ON, Canada, ${ }^{17}$ Research Centre for Medical Genetics, Moscow, Moscow, Russian Federation, ${ }^{18}$ Department of Neurology, University Hospital Bonn, Bonn, Germany, ${ }^{19}$ Department of Medical Chemistry, Molecular Biology and Pathobiochemistry, Semmelweis University, Budapest, Hungary, ${ }^{20}$ Department of Experimental Epileptology and Cognition Research, University of Bonn, Bonn, Germany, ${ }^{21}$ Bavarian Center for Biomolecular Mass Spectrometry (BayBioMS), Technische Universität München, München, Germany, ${ }^{22}$ Institute for Cardiovascular Physiology, Goethe-University, Frankfurt am Main, Germany, ${ }^{23}$ Department of Pediatrics, Salzburger Landeskliniken and Paracelsus Medical University Salzburg, Salzburg, Austria, ${ }^{24}$ Sackler Faculty of Medicine, Tel-Aviv University, Tel-Aviv, Israel, ${ }^{25}$ Ophthalmology Department, Centre National d'Ophtalmologie des Qinze-Vingts, Paris, France

Leber's hereditary optic neuropathy (LHON) is one of the most frequent and first genetically defined mitochondrial diseases. The majority of LHON cases are caused by mutations in mitochondrial DNA-encoded complex I (CI) genes and are characterized by a mild CI defect. However, a significant number still remain genetically undefined. Utilizing human genetics and tracking of protein turnover in mitochondrial supercomplexes, we delineate the role of an orphan chaperone protein in a novel mechanism of CI function and describe a recessive form of LHON resulting from defects in this protein. Biallelic recessive DNAJC 30 mutations were discovered in LHON cases from 29 families, breaking the longstanding dogma of exclusive maternal inheritance. In 27 of the families, the variant is ascribed to an Eastern European founder event. Moreover, incomplete penetrance and male predominance hallmarking maternally inherited LHON are recapitulated, indicating that these features originate downstream of the genetic defect. Given the combination of variable penetrance and higher than expected allele frequency of the founder variant, the discovery proved challenging to current diagnostic approaches. Our experimental data from patient-derived fibroblast cell lines indicate that the pathology underpinning the disease, a mild but consistent $\mathrm{CI}$ defect, is due to reduced exchange of subunits of the CI N-module, responsible for the first part of electron transport and exposed to higher risk of damage. The identification of this repair system is of general biological importance, and may open promising avenues for future therapeutic interventions for defective CI. Grant references: 01GM1906D, 01GM1906A, 01GM1603, SFB 815/Z1, GR-2016-02361449, EXC2026.

S. L. Stenton: None. N. Sheremet: None. C. Catarino: None. N. Andreeva: None. Z. Assouline: None. P. Barboni: None. O. Barel: None. I. Bychkov: None. L. Caporali: 
None. P. Charbel Issa: None. P. Freisinger: None. S. Gerber: None. D. Ghezzi: None. J. Heidler: None. M. Hempel: None. E. Heon: None. Y. Itkis: None. E. Javasky: None. J. Kaplan: None. R. Kopajtich: None. C. Kornblum: D. Speakers Bureau/Honoraria (speakers bureau, symposia, and expert witness); Modest; Santhera Pharmaceuticals. R. Kovacs-Nagy: None. T. Krylova: None. W. Kunz: None. C. La Morgia: None. C. Lamperti: None. C. Ludwig: None. P. Malacarne: None. J. Mayr: None. J. Meisterknecht: None. T. Nevinitsyna: None. F. Palombo: None. B. PodeShakked: None. M. Shmelkova: None. M. Tzadok: None. A. van der Ven: None. C. Vignal-Clermont: None. M. Wagner: None. E. Zakharova: None. N. Zhorzholadze: None. J. Rozet: None. V. Carelli: None. P. Tsygankova: None. T. Klopstock: B. Research Grant (principal investigator, collaborator or consultant and pending grants as well as grants already received); Modest; Santhera Pharmaceuticals and GenSight Biologics. D. Speakers Bureau/ Honoraria (speakers bureau, symposia, and expert witness); Modest; Santhera Pharmaceuticals and GenSight Biologics. I. Wittig: None. H. Prokisch: None.

\section{C25.6}

\section{WES profiling of COVID-19}

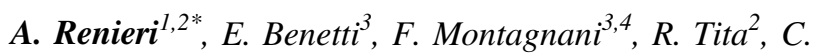
Fallerini $^{1}$, S. Amitrano ${ }^{2}$, M. Bruttini ${ }^{2}$, G. Doddato ${ }^{1}$, A.

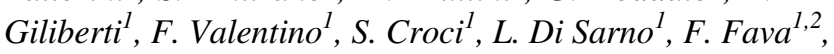
M. Baldassarri ${ }^{1}$, A. Tommasi ${ }^{1,2}$, M. Palmieri ${ }^{l}, A$. Emiliozzi $^{3,4}$, M. Fabbiani ${ }^{4}$, B. Rossetti ${ }^{4}$, G. Zanelli ${ }^{3,4}$, L. Bergantini $^{5}$, M. D'Alessandro ${ }^{5}$, P. Cameli $^{5}$, D. Bennet $^{5}, F$. Anedda $^{6}$, S. Marcantonio ${ }^{6}$, S. Scolletta ${ }^{6}$, F. Franchi ${ }^{6}$, M. Mazzei $^{7}$, E. Conticini ${ }^{8}$, L. Cantarini ${ }^{9}$, B. Frediani ${ }^{10}$, D. Tacconi $^{11}$, M. Feri ${ }^{12}$, R. Scala ${ }^{13}$, A. Ognibene ${ }^{14}, G$. Spargi $^{15}$, C. Nencioni ${ }^{16}$, G. Caldarelli ${ }^{17}$, M. Spagnesi ${ }^{18}, A$. Canaccini $^{19}$, E. Frullanti ${ }^{1}$, I. Meloni ${ }^{1}$, M. Mencarelli ${ }^{2}, C$. Lo Rizzo ${ }^{2}$, A. Pinto ${ }^{2}$, E. Bargagli ${ }^{5}$,M. Mandalà ${ }^{20}, S$. Furini $^{3}$, F. Mari $^{1,2}$

${ }^{1}$ Medical Genetics, University of Siena, Siena, Italy, ${ }^{2}$ Genetica Medica, Azienda Ospedaliera Universitaria Senese, Siena, Italy, ${ }^{3}$ Dept of Medical Biotechnologies, University of Siena, Siena, Italy, ${ }^{4}$ Dept of Specialized and Internal Medicine, Tropical and Infectious Diseases Unit, Siena, Italy, ${ }^{5}$ Unit of Respiratory Diseases and Lung Transplantation, Department of Internal and Specialist Medicine, University of Siena, Siena, Italy, ${ }^{6}$ Dept of Emergency and Urgency, Medicine, Surgery and Neurosciences, Unit of Intensive Care Medicine, Siena University Hospital, Siena, Italy, ${ }^{7}$ Dept of Medical, Surgical and Neuro Sciences, Diagnostic Imaging, University of Siena, Azienda Ospedaliera Universitaria Senese, Siena, Italy, ${ }^{8}$ Rheumatology Unit, Department of Medicine, Surgery and
Neurosciences, University of Siena, Policlinico Le Scotte, Siena, Italy, ${ }^{9}$ Dept of Medical Sciences, University of Siena, Siena, Italy, ${ }^{10}$ Research Center of Systemic Autoinflammatory Diseases and Behçet's Disease and RheumatologyOphthalmology Collaborative Uveitis Center, Department of Medical Sciences, Surgery and Neurosciences, University of Siena, Siena, Italy, ${ }^{11}$ Dept of Specialized and Internal Medicine, Infectious Diseases Unit, San Donato Hospital Arezzo, Arezzo, Italy, ${ }^{12}$ Dept of Emergency, Anesthesia Unit, San Donato Hospital, Arezzo, Italy, ${ }^{13}$ Dept of Specialized and Internal Medicine, Pneumology Unit and UTIP, San Donato Hospital, Arezzo, Italy, ${ }^{14}$ Clinical Chemical Analysis Laboratory, San Donato Hospital, Arezzo, Italy, ${ }^{15}$ Dept of Emergency, Anesthesia Unit, Misericordia Hospital, Grosseto, Italy, ${ }^{16}$ Dept of Specialized and Internal Medicine, Infectious Diseases Unit, Misericordia Hospital, Grosseto, Italy, ${ }^{17}$ Clinical Chemical Analysis Laboratory, Misericordia Hospital, Grosseto, Italy, ${ }^{18}$ Dept of Prevention, ASL South-East Tuscany, ASL South-East Tuscany, Italy, ${ }^{19}$ Territorial Scientific Technician Department, ASL South-East Tuscany, ASL South-East Tuscany, Italy, ${ }^{20}$ Otolaryngology Unit, University of Siena, Siena, Italy

Introduction: Italy has been the first European Country experiencing the epidemic wave of SARS-CoV-2 infection reaching 200,000 cases. On March 16, we started the GENCOVID project (https://sites.google.com/dbm.unisi.it/gencovid) aiming to collect 2,000 COVID-19 patients all over Italy, the first 500 being already collected.

Material and Methods: A first cohort of 130 Tuscanian COVID-19 patients (100 of which hospitalised) was subjected to clinical and molecular characterisation by Whole Exome Sequencing (WES).

Results: Searching for common genes by Collapsing Methods against 300 WES controls of Italian population failed to give straightforward statistically significant results with the exception of a pair of genes, one belonging to the Olfactory Receptor families with many paralogs in the genome. This result is not unexpected since we are facing the most challenging common disorder triggered by environmental factor with a strong underlying heritability (50\%). Learning the lesson of Autism Spectrum Disorder, we started to re-analyse the cohort treating each patient as an independent case, following a Mendelian-like model. We identified for each patient an average of 3 pathogenic mutations involved in virus infection susceptibility and pinpoint to one or more rare disorder(s); the number being higher in the most severely affected cases. To our knowledge, this is the first report on WES and COVID-19. Our results pinpoint to a combined model for COVID-19 susceptibility with a number of, yet unknown, common susceptibility genes which represent the favorite background 
in which additional rare mutations confer to the host the best environment for virus growth and organ damage.

A. Renieri: None. E. Benetti: None. F. Montagnani: None. R. Tita: None. C. Fallerini: None. S. Amitrano: None. M. Bruttini: None. G. Doddato: None. A. Giliberti: None. F. Valentino: None. S. Croci: None. L. Di Sarno: None. F. Fava: None. M. Baldassarri: None. A. Tommasi: None. M. Palmieri: None. A. Emiliozzi: None. M. Fabbiani: None. B. Rossetti: None. G. Zanelli: None. L. Bergantini: None. M. D'Alessandro: None. P. Cameli: None. D. Bennet: None. F. Anedda: None. S. Marcantonio: None. S. Scolletta: None. F. Franchi: None. M. Mazzei: None. E. Conticini: None. L. Cantarini: None. B. Frediani: None. D. Tacconi: None. M. Feri: None. R. Scala: None. A. Ognibene: None. G. Spargi: None. C. Nencioni: None. G. Caldarelli: None. M. Spagnesi: None. A. Canaccini: None. E. Frullanti: None. I. Meloni: None. M. Mencarelli: None. C. Lo Rizzo: None. A. Pinto: None. E. Bargagli: None. M. Mandalà: None. S. Furini: None. F. Mari: None.

\section{C26 New Technologies and Approaches}

\section{C26.1}

\section{Electrical impedance detection based GenapSys sequencer enables diverse genomic applications}

\author{
S. Paliwal", A. Nabi, M. Fallahi, M. R. Barmi, M. Jouzi, S. \\ Stern, X. Gomes, H. Nezamfar, E. LoPrete, B. Dong, P. \\ Kenney, K. B. Parizi, H. Rategh, S. Sankar, H. \\ Esfandyarpour
}

\section{Genapsys Inc, Redwood City, CA, United States}

Next Generation Sequencing (NGS) technologies have made rapid strides in the throughput and accuracy of DNA sequencing in recent years. These advances have revolutionized biomedical and clinical research. Here, Genapsys presents a novel, scalable, low cost, and high accuracy NGS platform, which enables wide-ranging genomic applications.

The GenapSys NGS platform is based on accurate detection of electrical impedance changes resulting from single base incorporations during sequencing-by-synthesis. We show that impedance changes measure a steady state dNTP incorporation signal, leading to higher accuracy. The core of the technology is a CMOS-based electronic chip that enables scalability and low instrument and consumable costs. Chips with $1 \mathrm{M}, 16 \mathrm{M}$ and $144 \mathrm{M}$ sensors can be run on the same GenapSys instrument, giving a lab flexibility in NGS assay design and sample multiplexing. We demonstrate that a single run with a $16 \mathrm{M}$ sensor chip generates up to $2 \mathrm{~Gb}$ of high accuracy data, with greater than $99 \%$ raw accuracy and average read lengths of $150 \mathrm{bp}$.
We demonstrate wide-ranging genomic applications of the GenapSys platform, which include whole genome sequencing of small genomes such as bacteria and viruses, whole exome sequencing of human samples, and transcriptomic applications. We also demonstrate that the high accuracy of the GenapSys platform makes it ideal for germline and somatic variant calling for inherited diseases and cancer. Thus, the GenapSys NGS platform is an accurate, scalable, and low cost solution for genomics research and clinical applications.

S. Paliwal: A. Employment (full or part-time); Significant; Genapsys Inc. A. Nabi: A. Employment (full or parttime); Significant; Genapsys Inc. M. Fallahi: A. Employment (full or part-time); Significant; Genapsys Inc. M. R. Barmi: A. Employment (full or part-time); Significant; Genapsys Inc. M. Jouzi: A. Employment (full or part-time); Significant; Genapsys Inc. S. Stern: A. Employment (full or part-time); Significant; Genapsys Inc. X. Gomes: A. Employment (full or part-time); Significant; Genapsys Inc. H. Nezamfar: A. Employment (full or part-time); Significant; Genapsys Inc. E. LoPrete: A. Employment (full or part-time); Significant; Genapsys Inc. B. Dong: A. Employment (full or part-time); Significant; Genapsys Inc. P. Kenney: A. Employment (full or part-time); Significant; Genapsys Inc. K. B. Parizi: A. Employment (full or parttime); Significant; Genapsys Inc. H. Rategh: A. Employment (full or part-time); Significant; Genapsys Inc. S. Sankar: A. Employment (full or part-time); Significant; Genapsys Inc. H. Esfandyarpour: A. Employment (full or part-time); Significant; Genapsys Inc.

\section{C26.2}

A bespoke gene-to-patient approach uplifts novel gene discovery for rare disease diagnostics

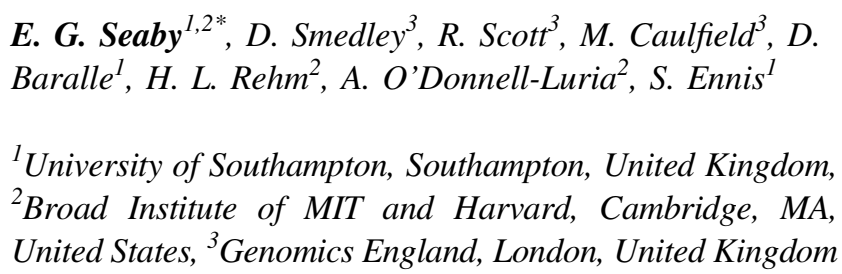

Next generation sequencing has revolutionised rare disease diagnostics. Concomitant with advancing technologies has been a rise in novel disease-gene discoveries. However, diagnostic rates remain suboptimal at $\sim 30 \%$. Novel gene discovery is still hindered by vast quantities of variants of uncertain significance found in genes of undetermined biological function. This necessitates intensive functional experiments on genes of equally-predicted causality that is proving a major bottleneck. Herein we propose a bespoke method that reverses the traditional approach of novel gene discovery. We apply the LOEUF metric of intolerance to 
gene inactivation to curate a list of predicted haploinsufficient disease genes. By leveraging genomic and phenotypic data from the 100,000 Genomes Project, we adopt a geneto-patient approach that matches de novo loss-of-function variants in constrained genes to rare disease patients, thus reducing most of the analytical noise that currently precludes novel discoveries. Preliminary data reveal the most constrained LOEUF genes $(n=1044)$ are highly correlated with OMIM disease genes. Yet, $70 \%$ have no disease association, leaving many probable disease genes yet to be discovered. Pilot data from the 100,000 Genomes Project $(n=28,000)$ reveal 655 rare (MAF <0.001), de novo loss-offunction events in 1044 LOEUF-constrained genes. 476 are in known OMIM genes and 179 are in novel genes (174 cases involving 138 genes). Of these, 27 genes have more than one kindred affected and 17 have the same/similar phenotypes. Therefore, rethinking current practices by utilising large cohorts and adopting gene-based approaches can accelerate novel gene discovery and target the most appropriate genes for functional validation.

E. G. Seaby: None. D. Smedley: None. R. Scott: None. M. Caulfield: None. D. Baralle: None. H. L. Rehm: None. A. O'Donnell-Luria: None. S. Ennis: None.

\section{C26.3}

\section{Analysis of Mendelian phenotypes through mouse} embryo viability screening

\author{
P. Cacheiro $^{1 *}$, S. A. Murray ${ }^{2}$, V. Muñoz-Fuentes ${ }^{3}$, H. \\ Westerberg , M. E. Dickinson ${ }^{5}$, A. Mallon ${ }^{4}$, T. F. Meehan ${ }^{3}$, \\ D. Smedley ${ }^{l}$, the International Mouse Phenotyping \\ Consortium
}

${ }^{1}$ William Harvey Research Institute, Queen Mary University of London, London, United Kingdom, ${ }^{2}$ The Jackson Laboratory, Bar Harbor, ME, United States, ${ }^{3}$ European Molecular Biology Laboratory, European Bioinformatics Institute, Hinxton, United Kingdom, ${ }^{4}$ Medical Research Council Harwell, Mammalian Genetics Unit and Mary Lyon Centre, Harwell, United Kingdom, ${ }^{5}$ Department of Molecular Physiology and Biophysics, Baylor College of Medicine, Houston, TX, United States

We have recently described a gene classification across the Full Spectrum of Intolerance to Loss-of-function (LoF) (FUSIL) by combining mouse viability screens and human cell proliferation assays, and showing specific enrichment of disease genes in discrete categories. Here, we further dissect this spectrum by assessing the embryonic stage at which homozygous LoF results in lethality in mice, and explore the correlation with human phenotypes.

Four hundred mouse lethal genes identified by the International Mouse Phenotyping Consortium were additionally classified into one of three windows of embryonic lethality: early, mid or late gestation. Early lethal genes showed a lower percentage of genes with abnormal phenotypes reported in the human orthologues when compared to late lethal genes $(40 \%$ vs $51 \%$, $\mathrm{P}=0.048$ ), while the percentage of disease genes associated with autosomal recessive inheritance was significantly higher (74 \% vs $46 \%, \mathrm{P}=0.0005$ ). Consistent with this observation, we found higher pRec scores among the early lethals (median pRec for early, mid and late windows: 0.80 , 0.50 and 0.36; median pLI: 0.004, 0.07 and 0.32). Neurological and neurodevelopmental were the most frequent type of diseases across windows (19\%, 24\% and $27 \%$ ). Skeletal disorders were the second most frequent class among those genes lethal at mid-gestation, which correlates with the organogenesis phase of embryonic development. Conversely, early lethal disease genes were highly enriched for metabolic disorders (OR 4.27, $\mathrm{P}=0.0003$ ).

The mouse embryonic development stage where LoF leads to lethality allows us to establish associations with human disease features. This knowledge may assist the prioritisation of candidate genes.

P. Cacheiro: None. S. A. Murray: None. V. MuñozFuentes: None. H. Westerberg: None. M. E. Dickinson: None. A. Mallon: None. T. F. Meehan: None. D. Smedley: None.

\section{C26.4}

Patient-derived retinal organoids to model retinitis pigmentosa 1 (RP1)

\section{P. Berber*, A. Milenkovic, C. Brandl, B. H. F. Weber}

\section{University of Regensburg, Regensburg, Germany}

Introduction: Human induced pluripotent stem cells (hiPSC) are an excellent starting point for generating 3D retinal organoids, which contain many cell types native to the retina. Still, retinal organoids lack critical physiological contact between photoreceptors and retinal pigment epithelium (RPE) cells. Nevertheless, retinal organoids offer exciting new pathways of scientific discovery, including modeling retinal dystrophies such as Retinitis Pigmentosa 1 (RP1, OMIM \#180100), where vision loss is caused by the degeneration of photoreceptors. Here, we have differentiated retinal organoids from healthy controls and RP1 patients harboring autosomal dominant mutations in the RP1 gene (OMIM \# 603937).

Materials and Methods: Human dermal fibroblasts were obtained from skin biopsies of six RP1 patients and healthy controls, and reprogrammed into hiPSCs. An organoid differentiation protocol was adapted from published 
protocols. A novel method to harvest and expand RPE cells as byproducts from the organoid differentiation protocol, was also established.

Results: Successful retinal organoid differentiation was confirmed, via immunocytochemistry staining for ganglion and photoreceptor markers. Photoreceptor differentiation was improved by culturing the organoids on an orbital shaker, facilitating the redistribution of fresh media and waste. Organoids differentiated from RP1 patient hiPSC showed a dramatic reduction in cells expressing recoverin, a developing photoreceptor marker $(13 \pm 4.35$ vs $80.3 \pm 4.51$, $\mathrm{p}$-value $\left.=4.92 * 10^{-05}\right)$. RPE cells were used to establish a co-culture with the organoids, to induce physiological contact between photoreceptors and RPE.

Conclusions: Retinal organoids can be differentiated from hiPSC, and provide an exciting new avenue of research to model inherited retinal dystrophies, including RP1.

P. Berber: None. A. Milenkovic: None. C. Brandl: None. B. H. F. Weber: None.

\section{C26.6}

Next generation cytogenetics: genome-imaging improves structural variant detection for constitutional chromosomal aberrations

K. Neveling*, T. Mantere, E. Kater-Baats, R. van Beek, M. Oorsprong, S. Vermeulen, M. Pauper, D. Olde Weghuis, M. Stevens-Kroef, G. van der Zande, D. Smeets, A. Hoischen

\section{Department of Human Genetics, Nijmegen, Netherlands}

Recently, we raised the question whether the Bionano genomeimaging technology would have potential to replace all, FISH, karyotyping and $\mathrm{CNV}$-microarray studies, in the analysis of somatic aberrations for different kind of leukemia samples. ${ }^{1}$ Here we aimed to test the clinical utility of this technology for the analysis of constitutional aberrations in DNA derived from various tissues. We analyzed samples with known aberrations of patients with infertility, recurrent abortions or intellectual disability, as well as prenatal samples by genome imaging.

For 30 samples, ultra-high molecular weight gDNA was isolated either from blood (EDTA/Heparin) or cell lines (fibroblasts/amniotic fluid/chorionic villi). DNA was processed and imaged on the Saphyr instrument (Bionano Genomics). ${ }^{1}$ For each sample at least $100 \mathrm{x}$ genome coverage was obtained. Using Bionano Access software, de novo genome assembly, SV and CNV variant calling and annotation was performed.

Sample preparation for all different materials was successful. Data analysis on average resulted in $\sim 50$ rare SVs and $\sim 20$ high confidence $\mathrm{CNVs}$ per sample. In the analyzed cases, all previously clinically reported structural aberrations were detected except for Robertsonian translocations, as expected for the current technology. Correctly detected aberrations included 8 microdeletions and 8 duplications, 11 translocations, 1 inversion and 4 aneuploidies.

Bionano genome-imaging is a promising technology with potential to become a generic cytogenetic test for all types of (molecular-)cytogenetic aberrations. The 10.000-time higher resolution as compared to standard karyotyping thereby enables precise breakpoint mapping and strikingly, even balanced translocations and inversions are picked up easily.

1. Neveling et al. (2020). bioRxiv-https://doi.org/10. 1101/2020.02.06.935742

K. Neveling: None. T. Mantere: None. E. Kater-Baats: None. R. van Beek: None. M. Oorsprong: None. S. Vermeulen: None. M. Pauper: None. D. Olde Weghuis: None. M. Stevens-Kroef: None. G. van der Zande: None. D. Smeets: None. A. Hoischen: None.

\section{C27 Genome Variation and Architecture}

\section{C27.1}

NCBI ALFA: Providing Sequence Variation Resource from 1 Million dbGaP Subjects

\section{Phan ${ }^{*}, N C B I d b S N P, N C B I d b G a P$}

\section{NCBI, Bethesda, MD, United States}

The aim of the NCBI Allele Frequency Aggregator (ALFA) project (https://go.usa.gov/xdNvF) is to make frequency data from over 1M subjects in the database of Genotype and Phenotype (dbGaP), previously under authorized access, available for open-access to facilitate discoveries and interpretations of variants with biological impacts or causing diseases. The ALFA project will compute allele frequency for variants across approved un-restricted dbGaP studies. This involved harmonizing and normalizing Heterogeneous data and file formats from GWAS chip array genotyping, exomes, and whole genomes. were QC and transformed into standard VCF format as input into an automated pipeline using the new SPDI notation (Holmes et al., 2019) to aggregate, remap and cluster to existing dbSNP rs, and computed allele frequency. Allele frequencies are computed for 12 major populations including European, Hispanic, African, Asian, and other diverse population ancestries that were determined using GRAF-pop software developed by dbGaP (Jin et al., 2019). The data is integrated with dbSNP 680 million Reference SNP (rs) and existing allele frequency reported for over 500 million rs from various projects including 1000Genomes, ExAc, GnomAD, TopMed, and many other population studies. The initial ALFA aggregate data release is from 110 thousand dbGaP subjects, $\sim 10 \%$ of available data. Aggregated from 677 billion genotypes, the results included allele counts and frequency for 531 million known dbSNP rs site and 18 million novel ones. 
Subsequent quarterly releases will include additional new $\mathrm{dbGaP}$ studies and combined can reach over a billion variants from millions of subjects.

L. Phan: None.

\section{C27.2}

Predicting the effects of structural variants in the human genome

\section{P. Kleinert ${ }^{*}$, M. Kircher}

\section{Berlin Institute of Health (BIH), Berlin, Germany}

In recent years, we saw rapid technological advances in the identification of structural variants (SVs). However, the interpretation of these variants remains challenging. Several methods were developed that utilize individual mechanistic principles like the deletion of coding sequence or 3D genome architecture disruptions. However, a comprehensive tool that uses the broad spectrum of available annotations for estimating the effect of SVs and prioritize functional variants in health and disease is missing. Here we describe CADD-SV, a method to retrieve and integrate a wide set of annotations across the range and in the vicinity of SVs for functional scoring. So far, supervised learning approaches were of very limited power for this kind of application, due to a very small number of functionally annotated (e.g. pathogenic/benign) SV sets. We overcome this problem by using a surrogate training-objective, the combined annotation dependent depletion (CADD) of functional variants in evolutionary derived variant sets. Our tool computes summary statistics and uses a trained linear model to differentiate deleterious from neutral structural variants. We use human and chimpanzee derived alleles as proxy-neutral and contrast them with matched simulated variants as proxypathogenic, an approach that has proven powerful in the interpretation of SNVs and short InDels (Kircher \& Witten et al, 2014). In a proof-of-principle study, we show that CADD-SV scores correlate with known pathogenic variants in individual genomes as well as allelic diversity observed across the population. The ability of the current model to prioritize functionally relevant and pathogenic variants is unmatched by existing methods.

P. Kleinert: None. M. Kircher: None.

\section{C27.3}

RNA secondary structure mediated by $A l u$ insertion as a novel disease-causing mechanism

E. Masson $^{1,2}$, S. Maestri ${ }^{1,2}$, D. N. Cooper ${ }^{3}$, C. Férec ${ }^{1,2}$, J. M. Chen ${ }^{1 *}$
${ }^{1}$ Univ Brest, Inserm, EFS, UMR 1078, GGB, Brest, France, ${ }^{2}$ CHRU Brest, Service de Génétique Médicale et de Biologie de la Reproduction, Brest, France, ${ }^{3}$ Institute of Medical Genetics, School of Medicine, Cardiff University, Cardiff, United Kingdom

We have recently reported a homozygous Alu insertion variant (termed Alu_Ins) within the $3^{\prime}$-untranslated region $\left(3^{\prime}\right.$ UTR) of the SPINKI gene as the cause of a new pediatric disease entity. Although Alu_Ins had been shown, by means of a cell culture-based full-length gene expression assay (FLGEA; expression vector contained SPINK1's 7-kb entire coding and intronic sequences), to result in the complete loss of SPINKI mRNA expression, the precise underlying mechanism(s) has remained elusive. Herein, we filled this knowledge gap by adopting a hypothesis-driven approach. Employing RepeatMasker, we identified two Alu elements (termed Alu1 and Alu2) within the SPINK1 locus; both are located deep within intron 3 and, most importantly, reside in the opposite orientation to Alu-Ins. We then performed the cell culture-based FLGEA on a total of 7 full-length SPINK1 expression vectors (i.e., wild-type (WT), mutant (Mut), WT_delAlu1, Mut_delAlu1, WT_delAlu2, Mut_delAlu2 and inversion of $A l u \_$Ins) in the presence of a nonsense-mediated mRNA decay inhibitor, cycloheximide. The results clearly demonstrated that $A l u \_I n s$ disrupts splicing by forming RNA secondary structures with $A l u 1$ in the pre-mRNA sequence rather than by its primary sequence. This is the first time that an $A l u$ insertion variant has been shown to exert its effect via such a mechanism causing human genetic disease. Given the abundance of pre-existing $A l u$ elements within the human genome and the potential insertion of new Alu elements into virtually any site, our findings have important implications for the detection and interpretation of $A l u$ elements in human disease genes. (This work was supported by INSERM).

E. Masson: None. S. Maestri: None. D. N. Cooper: None. C. Férec: None. J. M. Chen: None.

\section{C27.4}

Increased mutation rates and genome variation at active spermatogonial regulatory sites

\author{
V. B. Kaiser ${ }^{*}$, L. Talmane, Y. Kumar, F. Semple, M. \\ MacLennan, Deciphering Developmental Disorders Study, \\ D. R. FitzPatrick, M. S. Taylor, C. A. Semple
}

MRC Human Genetics Unit, Edinburgh, United Kingdom

Introduction: The germline is the ultimate source of genetic variation, but we know little about the influence of germline chromatin structure on the processes that give rise to mutations. Here, we use germline epigenomic data to study the influence of transcription factor binding sites 
(TFBSs) in the germline on the patterns of different classes of mutation across the human genome.

Materials and Methods: We use novel spermatogonial ATAC-seq data to infer the locations of TFBSs and meiotic recombination initiation sites (PRDM9 binding sites). We then relate these sites to structural variants in the DDD (Deciphering Developmental Disorders) cohort, very rare (gnomAD database) variants from extant human populations, and de novo mutations.

Results: Germline TFBSs are impacted by significantly increased rates of structural variant breakpoints: $32 \%$ of singleton DDD breakpoints are within $1 \mathrm{~KB}$ of an ATACSeq peak, and particular testis-active factors are enriched. Both de novo and rare gnomAD short insertions are enriched at the same TFBSs, suggesting these sites are particularly susceptible to mutation and that they employ an unusual DNA repair mechanism. The increased mutation rates associated with germline TF binding disproportionately affect a class of enhancers active in human neurodevelopment, since these enhancers overlap TFBSs active in spermatogonia (odds ratio of mutation $=6.82$, 95\% CI $=[5.34,8.71]$ for deletion breakpoints).

Conclusion: Spermatogonial TFBSs drive genome increased mutation rates and regulatory redundancy may lead to neurodevelopmental disease.

V. B. Kaiser: None. L. Talmane: None. Y. Kumar: None. F. Semple: None. M. MacLennan: None. D. R. FitzPatrick: None. M. S. Taylor: None. C. A. Semple: None.

\section{C27.5}

Resolving complex structural variants in recurrent brain tumors

K. Okonechnikov ${ }^{1,2}, J$. Hübner ${ }^{1,2}$, O. Chapman ${ }^{3}, A$. Chakraborty ${ }^{4}$, M. Pagadal ${ }^{3}$, R. Bumb ${ }^{5}$, S. Chandran $^{5}, K$. Kraft $^{6}$, R. Hidalgo ${ }^{7}$, S. Mundlos ${ }^{7}$, R. Wechsler-Reya ${ }^{8}, N$. Coufal $^{9}$, M. Levy ${ }^{10}$, J. Crawford ${ }^{11}$, K. Pajtler ${ }^{1,2,12}$, D. Reid $^{13}$, A. Schmitt ${ }^{13}$, H. Carter ${ }^{3}, F . A y^{4}, J$. Dixon ${ }^{5}, J$. Mesirov $^{3,14}$, S. M. Pfister ${ }^{1,2,12}$, M. Kool ${ }^{1,2}$, L. Chavez $^{3,14 *}$

${ }^{1}$ Hopp Children's Cancer Center at the NCT Heidelberg (KiTZ), Heidelberg, Germany, ${ }^{2}$ Division of Pediatric Neurooncology, German Cancer Research Center (DKFZ), Heidelberg, Germany, ${ }^{3}$ Department of Medicine, University of California San Diego (UCSD), San Diego, CA, United States, ${ }^{4}$ Division of Vaccine Discovery, La Jolla Institute for Allergy and Immunology, La Jolla, CA, United States, ${ }^{5}$ Salk Institute for Biological Studies, La Jolla, CA, United States, ${ }^{6}$ Center for Personal Dynamic Regulomes, Stanford University, Stanford, Stanford, CA, United States, ${ }^{7}$ Max Planck Institute for Molecular Genetics, Berlin, Germany, ${ }^{8}$ Tumor Initiation and Maintenance Program, NCI-Designated Cancer Center, Sanford Burnham Prebys Medical Research
Discovery Institute, La Jolla, CA, United States, ${ }^{9}$ Department of Pediatrics, University of California, San Diego, San Diego, CA, United States, ${ }^{10}$ Department of Neurosurgery, University of California San Diego - Rady Children's Hospital, Sam Diego, CA, United States, ${ }^{11}$ Department of Neurosciences, University of California San Diego - Rady Children's Hospital, San Diego, CA, United States, ${ }^{12}$ Department of Pediatric Oncology, Hematology and Immunology, Heidelberg University Hospital, Heidelberg, Germany, ${ }^{13}$ Arima Genomics, Inc, San Diego, CA, United States, ${ }^{14}$ Moores Cancer Center, University of California San Diego (UCSD), La Jolla, CA, United States

Introduction: Brain and other tumors of the CNS are the most common cancers in children aged 0-14 years in the USA. Ependymoma is the third most common pediatric brain tumor and a leading cause of death in childhood cancer patients. The most common and aggressive subgroup, posterior fossa ependymoma group A (PFA), occurs in young children and frequently leads to recurrences. Despite extensive DNA sequencing studies, the only molecular marker associated with particularly poor survival is gain of chromosome 1q.

Materials and Methods: Through profiling enhancers in ependymoma tumors, we have recently identified putative oncogenes, molecular targets, and functional pathways (Mack et al, Nature 2018). Complementary to the analysis of enhancers, we have now mapped the 3D organization of tumor chromatin in primary and relapse ependymoma tumors using $\mathrm{HiC}$.

Results: By an integrative analysis of enhancer and gene expression in the context of the newly derived $\mathrm{HiC}$ data, we identify new tumor-dependency genes activated by tumorspecific chromatin clusters of regulatory elements. We have also leveraged the $\mathrm{HiC}$ data for resolving structural variants (SVs) underlying copy number alterations. As a result, we identify complex inter-chromosomal SVs that result in the formation of new topologically associated domains ("neoTADs') leading to transcriptional activation of putative oncogenes.

Conclusions: We propose a new molecular mechanism by which chromosome 1q gains lead to complex interchromosomal SVs. By forming neo-TADs, these SVs lead to the transcriptional activation of oncogenes and thus potentially contribute to resistance in a subgroup of very aggressive pediatric brain tumors.

K. Okonechnikov: None. J. Hübner: None. O. Chapman: None. A. Chakraborty: None. M. Pagadal: None. R. Bumb: None. S. Chandran: None. K. Kraft: None. R. Hidalgo: None. S. Mundlos: None. R. Wechsler-Reya: None. N. Coufal: None. M. Levy: None. J. Crawford: None. K. Pajtler: None. D. Reid: None. A. Schmitt: None. H. Carter: 
None. F. Ay: None. J. Dixon: None. J. Mesirov: None. S. M. Pfister: None. M. Kool: None. L. Chavez: None.

\section{C28 Neurodevelopment}

\section{C28.1}

Pathogenic variants in RLIM/RNF12 lead to syndromic $\mathrm{X}$-linked intellectual disability and congenital, neurologic and behavior disorders

S. G. M. Frints ${ }^{1,2 *}$, A. Ozanturk ${ }^{3}$, G. Rodriguez Criado ${ }^{4}, U$. Grasshoff , M. Field ${ }^{6}$, S. Manouvrier-Hanu ${ }^{7,8}$, S. Hickey ${ }^{9,10}$, K. Gripp ${ }^{11}$, C. Bauer ${ }^{5}$, C. Schroeder ${ }^{5}$, A. Toutain ${ }^{12,13}, T$. Mihalic Mosher ${ }^{9,14}$, B. J. Kelly ${ }^{14}$, P. White ${ }^{9,14}$, A. Dufke $e^{5}, S$. Moon $^{3}$, D. C. Koboldt ${ }^{9,14}$, E. Gerkes ${ }^{15}$, A. van Haeringen ${ }^{16}$, C. Ruivenkamp ${ }^{16}$, C. Mignot ${ }^{17}$, B. Keren ${ }^{18}$, A. Müller ${ }^{5}, S$. Waldmüller $^{5}$, M. Blandfort ${ }^{19}$, U. Korda $\beta^{20}$, K. $_{\text {Gardner }}{ }^{21}$, A. Trimouille $^{22}$, K. E. P. van Roozendaal ${ }^{1,2}$, S. A. Haas ${ }^{23}, L$.

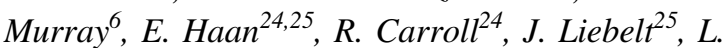
Hobson $^{26}$, M. De Rademaeker ${ }^{27}$, H. Brunner ${ }^{1,2,28}$, J. Glatz $^{1,2}$, K. Devriendt ${ }^{29}$, J. Vermeesch ${ }^{29}$, M. Raynaud $^{12,13,30}$, O. Riess ${ }^{5}$, J. Gribnau ${ }^{31}$, N. Katsanis ${ }^{3}$, P. Bauer ${ }^{5}$, J.

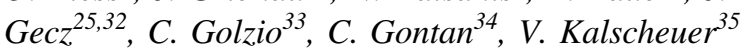

${ }^{1}$ Department of Clinical Genetics, Maastricht University Medical Center +, MUMC+, azM, Maastricht, Netherlands, ${ }^{2}$ Department of Genetics and Cell Biology, FHML, Maastricht University, Maastricht, Netherlands, ${ }^{3}$ Center for Human Disease Modeling and Departments of Pediatrics and Psychiatry, Duke University, Durham, NC, United States, ${ }^{4}$ Unidad de Genética Clínica, Hospital Virgen del Rocío, Sevilla, Spain, ${ }^{5}$ Institute of Medical Genetics and Applied Genomics, University of Tübingen, Tübingen, Germany, ${ }^{6}$ GOLD (Genetics of Learning and Disability) Service, Hunter Genetics, Waratah, Australia, ${ }^{7}$ Clinique de Génétique médicale Guy Fontaine, Centre de référence maladies rares Anomalies du développement Hôpital Jeanne de Flandre, Lille, France, ${ }^{8}$ Département Universitaire de Génétique, EA RADEME Maladies Rares du Développement et du Métabolisme, Faculté de Médecine, Université Lille 2, Lille, France, ${ }^{9}$ Division of Molecular \& Human Genetics, Nationwide Children's Hospital, Columbus, OH, United States, ${ }^{10}$ Department of Pediatrics, The Ohio State University College of Medicine, Ohio, $\mathrm{OH}$, United States, ${ }^{11}$ Alfred I. duPont Hospital for Children Nemours, Wilmington, DE, United States, ${ }^{12}$ Service de Génétique, Hôpital Bretonneau, CHU de Tours, Tours, France, ${ }^{13}$ UMR INSERM U930, Faculté de Médecine, Université François Rabelais, Tours, France, ${ }^{14}$ The Institute for Genomic Medicine, Nationwide Children's Hospital, Columbus, OH, United States, ${ }^{15}$ Department of Clinical Genetics, University Medical Center Groningen, UMCG, Groningen, Netherlands, ${ }^{16}$ Department of Clinical
Genetics, Leiden University Medical Center, LUMC, Leiden, Netherlands, ${ }^{17}$ Unité Génétique Clinique, Hôpital Armand-Trousseau, Paris, France, ${ }^{18}$ Genetic Department, Pitié-Salpêtrière Hospital, APHP. Sorbonne Université, Paris, France, ${ }^{19}$ Praxis für Neuropädiatrie und humangenetische Beratung, Landau, Germany, ${ }^{20}$ MVZ für Humangenetik und Molekularpathologie GmbH, Greifswald, Germany, ${ }^{21}$ RareREACH Research Patient Liaison, Rare Genomics Institute, 4859 West Slauson Avenue \#573, Los Angeles, CA 90056, USA, Los Angeles, CA, United States, ${ }^{22}$ Service de Génétique Médicale, CHU Bordeaux, Laboratoire MRGM, INSERM U1211, Univ., Bordeaux, France, ${ }^{23}$ Department of Computational Molecular Biology, Max Planck Institute for Molecular Genetics, Berlin, Germany, ${ }^{24}$ Adelaide Medical School and Robinson Research Institute, The University of Adelaide, Adelaide, Australia, ${ }^{25}$ South Australian Clinical Genetics Service, SA Pathology (at Royal Adelaide Hospital), Adelaide, Australia, ${ }^{26}$ Genetics and Molecular Pathology, SA Pathology, Adelaide, Australia, ${ }^{27}$ Centre for Medical Genetics, Reproduction and Genetics, Reproduction Genetics and Regenerative Medicine, Free University Brussel (VUB), UZ Brussel, Brussels, Belgium, ${ }^{28}$ Radboud University Medical Center, Department of Human Genetics, Nijmegen, Netherlands, ${ }^{29}$ Center for Human Genetics, University Hospitals Leuven, Leuven, Belgium, " ${ }^{30}$ Inserm U930 "Imaging and Brain", Tours, France, ${ }^{31}$ Department of Developmental Biology, Oncode Institute, Erasmus MC, University Medical Center, Rotterdam, Netherlands, ${ }^{32}$ South Australian Health and Medical Research Institute, Adelaide, Australia, ${ }^{33}$ U964; Université de33Institut de Génétique et de Biologie Moléculaire et Cellulaire, Centre National de la Recherche Scientifique, Université de Strasbourg, Illkirch-Graffenstaden, France, ${ }^{34}$ Department of Developmental Biology, Oncode Institute, Erasmus MC, University Medical Center Rotterdam, Rotterdam, Netherlands, ${ }^{35}$ Research Group Development and Disease, Max Planck Institute for Molecular Genetics, Berlin, Germany

Introduction: RLIM/RNF12 is an X-linked E3 ubiquitin ligase acting as a negative regulator of LIM-domain containing transcription factors and participates in X-chromosome inactivation $(\mathrm{XCI})$ in mice.

Material and Methods: We performed exome sequencing, in vitro and in vivo studies. Clinical genetic (re)evaluation was performed using a Human Phenotype Ontology scoring list. Methylation-based assays were used for determining $\mathrm{X}$ chromosome inactivation in carrier females.

Results: We will provide a review on affected males and carrier females from 9 families which we published recently and on 10 novel families who have unique missense variants in RLIM. All surviving affected males presented with X-linked intellectual disability (XLID) disorders. Ten 
percent of the heterozygous carrier females showed mild physical features. X-chromosome inactivation was skewed in $>91 \%$ of the carrier females. Most of the RLIM variants identified are predicted to affect protein function. Nine altered amino acids are conserved and lie within a domain essential for binding interacting proteins or in the Cterminal RING-finger catalytic domain. In vitro experiments revealed that the amino acid changes investigated impaired RLIM ubiquitin ligase activity. In vivo experiments in rlim mutant zebrafish showed that wild-type RLIM rescued the zebrafish rlim microcephaly phenotype, whereas the so far 9/9 patient-specific missense variants tested failed to rescue the phenotype and thus represent likely severe loss of function mutations.

Conclusions: The identified spectrum of functionally investigated pathogenic RLIM missense variants (9/9) cause related XLID disorders and impaired ubiquitin ligase activity, suggesting that proper function of RLIM is required for normal development, cognition and behavior.

S. G. M. Frints: None. A. Ozanturk: None. G. Rodriguez Criado: None. U. Grasshoff: None. M. Field: None. S. Manouvrier-Hanu: None. S. Hickey: None. K. Gripp: None. C. Bauer: None. C. Schroeder: None. A. Toutain: None. T. Mihalic Mosher: None. B. J. Kelly: None. P. White: None. A. Dufke: None. S. Moon: None. D. C. Koboldt: None. E. Gerkes: None. A. van Haeringen: None. C. Ruivenkamp: None. C. Mignot: None. B. Keren: None. A. Müller: None. S. Waldmüller: None. M. Blandfort: None. U. Kordaß: None. K. Gardner: None. A. Trimouille: None. K. E. P. van Roozendaal: None. S. A. Haas: None. L. Murray: None. E. Haan: None. R. Carroll: None. J. Liebelt: None. L. Hobson: None. M. De Rademaeker: None. H. Brunner: None. J. Glatz: None. K. Devriendt: None. J. Vermeesch: None. M. Raynaud: None. O. Riess: None. J. Gribnau: None. N. Katsanis: None. P. Bauer: None. J. Gecz: None. C. Golzio: None. C. Gontan: None. V. Kalscheuer: None.

\section{C28.2}

De-novo and Biallelic pathogenic variants in NARS1 cause neurodevelopmental delay due to dominant negative and partial loss of function effect

E. O'Connor ${ }^{1}, A$. Manole $^{1}$, S. Efthymiou ${ }^{1 *}, M$. Mendes $^{2}, M$. Jennings $^{3}$, D. Jenkins ${ }^{4}$, M. Lopez ${ }^{5}, R$. Maroofian ${ }^{1}, V$.

Salpietro $^{1}$, S. Antonarakis ${ }^{6}$, J. Vincent ${ }^{7}$, T. B. Haack ${ }^{8}, F$.

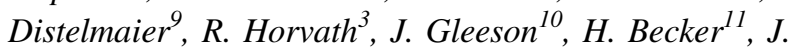
Mandel $^{12}$, D. Koolen ${ }^{13}$, H. Houlden ${ }^{1}$

${ }^{1}$ UCL Institute of Neurology, London, United Kingdom, ${ }^{2}$ Amsterdam University Medical Centers, Amsterdam, Netherlands, ${ }^{3}$ University of Cambridge, Cambridge, United Kingdom, ${ }^{4}$ Institute of Child Health, London, United Kingdom, ${ }^{5}$ Institute of Healthy Aging, London, United
Kingdom, ${ }^{6}$ University of Geneva, Geneva, Switzerland, ${ }^{7}$ Centre for Addiction and Mental Health, Ontario, ON, Canada, ${ }^{8}$ University of Tuebingen, Tuebingen, Germany, ${ }^{9}$ Heinrich-Heine-University, Dusseldorf, Germany, ${ }^{10}$ Howard Hughes Medical Institute, California, CA, United States, ${ }^{11}$ University of Strasbourg, Strasbourg, France, ${ }^{12}$ IGBMC, Strasbourg, France, ${ }^{13}$ Radboud University Medical Center, Nijmegen, Netherlands

Aminoacyl-tRNA-synthetases (ARSs) are ubiquitous, ancient enzymes that charge amino-acids to cognate tRNA molecules, the essential first-step of protein translation. Here, we describe 20 families consisting of 31 individuals with de-novo heterozygous and biallelic mutations in the asparaginyl-tRNA-synthetase gene (NARS1), with a phenotype characterised by microcephaly, neurodevelopmental delay, seizures, peripheral neuropathy and ataxia. NARS1 mRNA and protein expression from both patient fibroblasts and induced neural progenitor cells (iNPCs) were reduced; NARS1 enzyme levels in these tissues were also significantly reduced. Yeast complementation assay on the recessive p.Arg545Cys mutation was not detrimental, but molecular modelling of this mutation showed weaker spatial positioning and tRNA selectivity. Zebrafish modelling of the prevalent p.Arg534* de-novo mutation gave a severely abnormal locomotor phenotype. Here, we show that de-novo and biallelic mutations in NARSI are a prevalent cause of abnormal neurodevelopment, where the mechanism for denovo mutations is dominant negative and for recessive mutations partial loss-of-function. MRC Centre grant (G0601943)

E. O'Connor: None. A. Manole: None. S. Efthymiou: None. M. Mendes: None. M. Jennings: None. D. Jenkins: None. M. Lopez: None. R. Maroofian: None. V. Salpietro: None. S. Antonarakis: None. J. Vincent: None. T. B. Haack: None. F. Distelmaier: None. R. Horvath: None. J. Gleeson: None. H. Becker: None. J. Mandel: None. D. Koolen: None. H. Houlden: None.

\section{C28.3}

Biallelic loss-of-function variants in TBC1D2B cause a neurodevelopmental disorder with seizures and gingival overgrowth

F. L. Harms ${ }^{1 *}$, P. Parthasarathy ${ }^{2}$, D. Zorndt ${ }^{l}, M$. Alawi $^{3}, S$. Fuchs $^{1}$, B. J. Halliday ${ }^{2}$, C. McKeown ${ }^{4}$, H. Sampaio ${ }^{5,6}$, N. Radhakrishnan $^{7}$, S. K. Radhakrishnan ${ }^{8}$, R. Sachdev ${ }^{4}$, S. P. Robertson $^{2}$, S. Nampoothiri ${ }^{9}$, K. Kutsche ${ }^{1}$

${ }^{1}$ Institute of Human Genetics, University Medical Center Hamburg-Eppendorf, Hamburg, Germany, ${ }^{2}$ Department of Women's and Children's Health, Dunedin School of Medicine, University of Otago, Dunedin, New Zealand, ${ }^{3}$ Bioinformatics Core, University Medical Center HamburgEppendorf, Hamburg, Germany, ${ }^{4}$ Centre for Clinical 
Genetics, Sydney Children's Hospital, Randwick, Australia,

${ }^{5}$ Department of Women and Children's Health, Randwick Campus, University of New South Wales, Randwick, Australia, ${ }^{6}$ Sydney Children's Hospital, Randwick, Australia, ${ }^{7}$ Department of Ophthalmology, Amrita Institute of Medical Sciences \& Research Centre, Cochin, India, ${ }^{8}$ Department of Neurology, Amrita Institute of Medical Sciences \& Research Centre, Cochin, India, ${ }^{9}$ Department of Pediatric Genetics, Amrita Institute of Medical Sciences \& Research Centre, Cochin, India

The family of Tre2-Bub2-Cdc16 (TBC)-domain containing GTPase activating proteins (RABGAPs) is not only known as key regulators of RAB GTPase activity, but also has GAP-independent functions. RAB GTPases are implicated in membrane trafficking pathways, such as vesicular trafficking. We report biallelic loss-offunction variants in $T B C 1 D 2 B$, encoding a member of the TBC/RABGAP family with yet unknown function, as the underlying cause of cognitive impairment, seizures, and gingival overgrowth in two individuals from unrelated families. $T B C 1 D 2 B$ mRNA amount was drastically reduced and the protein was absent in fibroblasts of one patient. In immunofluorescence analysis, ectopically expressed TBC1D2B co-localized with vesicles positive for RAB5, a small GTPase orchestrating early endocytic vesicle trafficking. In two independent $T B C 1 D 2 B$ CRISPR/Cas9 knockout HeLa cell lines that serve as cellular model of $T B C 1 D 2 B$ deficiency, epidermal growth factor internalization was significantly reduced compared with the parental HeLa cell line suggesting a role of TBC1D2B in early endocytosis. Serum deprivation of $T B C 1 D 2 B$-deficient HeLa cell lines caused a decrease in cell viability and an increase in apoptosis. Our data reveal that loss of $T B C 1 D 2 B$ causes a neurodevelopmental disorder with gingival overgrowth, possibly by deficits in vesicle trafficking and/or cell survival.

F. L. H. was supported by the Research Promotion Fund of the Faculty of Medicine (FFM) of the University Medical Center Hamburg-Eppendorf. This work was supported by grants from the Deutsche Forschungsgemeinschaft (KU 1240/10-1) to K. K. and from Curekids New Zealand to S.P.R.

F. L. Harms: None. P. Parthasarathy: None. D. Zorndt: None. M. Alawi: None. S. Fuchs: None. B. J. Halliday: None. C. McKeown: None. H. Sampaio: None. N. Radhakrishnan: None. S. K. Radhakrishnan: None. R. Sachdev: None. S. P. Robertson: None. S. Nampoothiri: None. K. Kutsche: None.

\section{C28.4}

De novo pathogenic variants in $C S N K 2 B$ cause a new intellectual disability-craniodigital syndrome distinguished from Poirier-Bienvenu neurodevelopmental syndrome

M. Asif ${ }^{1,2,3^{*}}$, E. Kaygusuz ${ }^{1,2,4}$, M. Shinawi ${ }^{5}$, A. Nickelsen $^{6}$, J. Hochscherf ${ }^{7}$, D. Lindenblatt ${ }^{7}$, A. A. Noegel ${ }^{1,3}, S$.

Tinschert $^{8}$, K. Niefind ${ }^{7}$, P. Fortugno ${ }^{9}, J$. Jose $^{6}$, F. Brancati $^{9,10}$, P. Nürnberg ${ }^{1,3}$, M. S. Hussain ${ }^{1,2,3}$

${ }^{I}$ Cologne Center for Genomics, University of Cologne, Cologne, Germany, ${ }^{2}$ Institute of Biochemistry I, Medical Faculty, University of Cologne, Cologne, Germany, ${ }^{3}$ Center for Molecular Medicine Cologne (CMMC), University of Cologne, Cologne, Germany, ${ }^{4}$ Institute of Human Genetics, University Medical Center Göttingen, Göttingen, Germany, ${ }^{5}$ Division of Genetics and Genomic Medicine, Department of Pediatrics, Washington University School of Medicine, St. Louis, MO, United States, ${ }^{6}$ Institute of Pharmaceutical and Medicinal Chemistry, Westphalian Wilhelms-University, Münster, Germany, ${ }^{7}$ Department of Chemistry, Institute of Biochemistry, University of Cologne, Cologne, Germany, ${ }^{8}$ Zentrum Medizinische Genetik, Medizinische Universität, Innsbruck, Austria, ${ }^{9}$ Istituto Dermopatico dell'Immacolata (IDI) IRCCS, Rome, Italy, ${ }^{10}$ Medical Genetics, Department of Life, Health and Environmental Science, University of L'Aquila, L'Aquila, Italy

Introduction: In a cohort of patients with intellectual disability, microcephaly, growth failure, facial and digital anomalies, we recently identified mutations in $C K A P 2 L$ as a cause of Filippi syndrome (FS). One of these patients was tested negative for $C K A P 2 L$ and had a slightly different phenotype.

Materials and Methods: To identify the causal variants, trio whole-exome sequencing was performed. To further explore the consequences of identified variants, pulldown assays, microscale thermophoresis, immunofluorescence and whole transcriptome and whole phosphoproteome (WPP) profiling were conducted.

Results: A pathogenic de novo CSNK2B variant was identified in the proband, while a second patient recruited through GeneMatcher showing similar phenotype harbored a de novo $C S N K 2 B$ variant on same codon. Both variants, $\mathrm{p}$. (Asp32Asn) and p.(Asp32His), caused an up-regulation of $C S N K 2 B$ expression at transcript and protein level, which resulted in impaired cross talk between $\alpha$ and $\beta$ subunits of casein kinase 2 (CK2). The effect of p.(Asp32His) was observed in the canonical Wnt signaling (CWS) and DNA damage response (DDR) pathways. In CWS, an impaired 
interaction of DVL3 with mutant CK2 $\beta$ and up-regulation as well as mislocalization of $\beta$-catenin in mutant LCLs was found. In DDR, $\gamma \mathrm{H} 2 \mathrm{AX}, 53 \mathrm{BP} 1$ and CHK1 was substantially increased in mutant LCLs. Additionally, RNA-seq data confirmed differential expression of genes involved in the aforementioned pathways. Finally, WPP showed hypophosphorylation of 344 substrates of CK2.

Conclusions: Our findings suggest that $C S N K 2 B$ is a novel candidate gene for a rare distinguished craniodigital syndrome. The underlying pathogenic variants exert their pleiotropic effects through a dysregulation of CWS and DDR.

M. Asif: None. E. Kaygusuz: None. M. Shinawi: None. A. Nickelsen: None. J. Hochscherf: None. D. Lindenblatt: None. A. A. Noegel: None. S. Tinschert: None. K. Niefind: None. P. Fortugno: None. J. Jose: None. F. Brancati: None. P. Nürnberg: None. M. S. Hussain: None.

\section{C28.5}

Biallelic and monoallelic variants in PLXNA1 cause a syndromic disorder with neurodevelopmental and oculo-cerebral anomalies

G. C. Dworschak ${ }^{1,2,3 *}, J$. Punetha ${ }^{4}$, J. C. Kalanithy ${ }^{1}$, E.

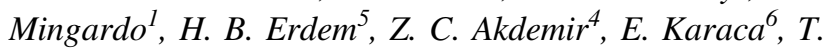
Mitani $^{4}$, D. Marafi, J. M. Fatih ${ }^{4}$, S. N. Jhangiani ${ }^{7}$, J. V. Hunter $^{8}$, T. Dakal ${ }^{9}$, B. Dhabhai ${ }^{9}$, O. Dabbagh ${ }^{10}$, H. S. Alsaif $^{11}$, F. S. Alkuraya ${ }^{11}$, R. Maroofian ${ }^{12}$, H. Houlden ${ }^{12}, S$. Efthymiou $^{12}$, N. Dominik ${ }^{12}$, V. Salpietro ${ }^{12}$, T. Sultan ${ }^{13}, S$. Haider $^{14}$, F. Bibi ${ }^{15}$, H. Thiele ${ }^{16}$, J. Hoefele ${ }^{17}$, K. M. Riedhammer $^{17}$, M. Wagner ${ }^{17}$, I. Guella ${ }^{18}$, M. Demos $^{19}$, B. Keren $^{20}$, J. Buratti ${ }^{20}$, P. Charles ${ }^{20}$, C. Nava ${ }^{20}$, E. Valkanas $^{21}$, L. B. Waddell ${ }^{22}$, K. J. Jones ${ }^{22}$, E. C. Oates ${ }^{22}$, S. T. Cooper $^{22}$, D. MacArthur ${ }^{23}$, R. E. Schnur ${ }^{24}$, G. E. Hoganson $^{25}$, J. E. Burton ${ }^{25}$, M. McEntagart ${ }^{26}$, D. Pehlivan ${ }^{4}$, J. E. Posey ${ }^{4}$, J. R. Lupski ${ }^{4,7}$, H. Reutter ${ }^{1,27}$

${ }^{1}$ Institute of Human Genetics, University of Bonn, Bonn, Germany, ${ }^{2}$ Institute of Anatomy, University of Bonn, Bonn, Germany, ${ }^{3}$ Department of Pediatrics, Children's Hospital, University of Bonn, Bonn, Germany, ${ }^{4}$ Department of Molecular and Human Genetics, Baylor College of Medicine, Houston, TX, United States, ${ }^{5}$ Department of Medical Genetics, University of Health Sciences, Diskapi Yildirim Beyazit Training and Research Hospital, Ankara, Turkey, ${ }^{6}$ Department of Genetics, University of Alabama at Birmingham, Birmingham, AL, United States, ${ }^{7}$ Human Genome Sequencing Center, Baylor College of Medicine, Houston, TX, United States, ${ }^{8}$ Department of Radiology, Baylor College of Medicine, Houston, TX, United States, ${ }^{9}$ Genome and Computational Biology Lab, Department of Biotechnology, Mohanlal Sukhadia University, Udaipur, India, ${ }^{10}$ Department of Neuroscience, King Faisal
Specialist Hospital and Research Center, Riyadh, Saudi Arabia, ${ }^{11}$ Department of Genetics, King Faisal Specialist Hospital and Research Centre, Riyadh, Saudi Arabia, ${ }^{12}$ Department of Neuromuscular Disorders, UCL Queen Square Institute of Neurology, University College London, London, United Kingdom, ${ }^{13}$ Department of Pediatric Neurology, Institute of Child Health, The Children's Hospital Lahore, Lahore, Pakistan, ${ }^{14}$ Department of Paediatric Medicine, Wah Medical College, Wah, Pakistan, ${ }^{15}$ University Institute of Biochemistry \& Biotechnology, PMAS - Arid Agriculture University, Rawalpindi, Pakistan, ${ }^{16}$ Cologne Center for Genomics, University of Cologne, Cologne, Germany, ${ }^{17}$ Institute of Human Genetics, Klinikum rechts der Isar, School of Medicine, Technical University of Munich, Munich, Germany, ${ }^{18}$ Department of Medical Genetics, Centre for Applied Neurogenetics, University of British Columbia, Vancouver, BC, Canada, ${ }^{19}$ Division of Neurology, Department of Pediatrics, University of British Columbia and BC Children's Hospital, Vancouver, BC, Canada, ${ }^{20} A P-H P$, Hôpital Pitié-Salpêtrière, Département de Génétique, Paris, France, ${ }^{21}$ Center for Mendelian Genomics, The Broad Institute of MIT and Harvard, Cambridge, MA, United States, ${ }^{22}$ Kids Neuroscience Centre, Kids Research, The Children's Hospital at Westmead, Sydney, Australia, ${ }^{23}$ Broad Institute of Harvard and Massachusetts Institute of Technology, Cambridge, MA, United States, ${ }^{24}$ GeneDx, Gaithersburg, MD, United States, ${ }^{25}$ Department of Pediatrics, University of Illinois, College of Medicine, Chicago, IL, United States, ${ }^{26}$ South West Thames Regional Genetics Centre, St. George's Healthcare NHS Trust, St. George's, University of London, London, United Kingdom, ${ }^{27}$ Department of Neonatology and Pediatric Intensive Care, University Hospital of Bonn, Bonn, Germany

PLXNA1 encodes plexin-A1, a neuronal transmembrane receptor for semaphorin signaling in the central nervous system that is suggested to play a key role in axon guidance. Here, we describe rare and novel potentially deleterious variants in PLXNA1 identified by exome sequencing in five families with biallelic variants and four families with monoallelic variants. Shared phenotypic features among affected individuals include global developmental delay (11/12), congenital brain anomalies (7/12), craniofacial dysmorphisms (7/12), and ocular anomalies (7/12). Notably, seizures were almost exclusively reported in individuals with monoallelic variants. Structural modeling of all missense variants in PLXNAI suggests distortion in the native protein. Knockdown of the zebrafish homolog plxnala in zebrafish larvae caused anomalies of the central nervous system and the eye supporting our observations in affected individuals. Our human genetic and functional studies in zebrafish indicate that PLXNAI plays an 
indispensable role in neurodevelopment and implicate it in a syndromic disorder with neurodevelopmental and oculocerebral anomalies. Grant: BONFOR grant O-120.0001 and Herbert-Reeck foundation (2019) to GCD

G. C. Dworschak: None. J. Punetha: None. J. C. Kalanithy: None. E. Mingardo: None. H. B. Erdem: None. Z. C. Akdemir: None. E. Karaca: None. T. Mitani: None. D. Marafi: None. J. M. Fatih: None. S. N. Jhangiani: None. J. V. Hunter: None. T. Dakal: None. B. Dhabhai: None. O. Dabbagh: None. H. S. Alsaif: None. F. S. Alkuraya: None. R. Maroofian: None. H. Houlden: None. S. Efthymiou: None. N. Dominik: None. V. Salpietro: None. T. Sultan: None. S. Haider: None. F. Bibi: None. H. Thiele: None. J. Hoefele: None. K. M. Riedhammer: None. M. Wagner: None. I. Guella: None. M. Demos: None. B. Keren: None. J. Buratti: None. P. Charles: None. C. Nava: None. E. Valkanas: None. L. B. Waddell: None. K. J. Jones: None. E. C. Oates: None. S. T. Cooper: None. D. MacArthur: None. R. E. Schnur: None. G. E. Hoganson: None. J. E. Burton: None. M. McEntagart: None. D. Pehlivan: None. J. E. Posey: None. J. R. Lupski: None. H. Reutter: None.

\section{C28.6}

Mutations in Gemin5 cause developmental delay, motor dysfunction and cerebellar atrophy

\section{U. Pandey ${ }^{*}$}

University of Pittsburgh Medical Center, Pittsburgh, PA, United States

GEMIN5, an RNA-binding protein is essential for assembly of the SMN complex. It facilitates the formation of small nuclear ribonucleoproteins (snRNPs), the building blocks of spliceosomes. GEMIN5 is involved in regulating the splicing of pre-mRNAs and has been shown to bind snRNAbinding protein of the SMN complex. Here we show identification of novel autosomal recessive variants in the GEMIN5 gene in 5 unrelated patient families with total 7 affected individuals. These variants have not been reported in any publicly available databases. The probands present with developmental delay, central hypotonia, ataxia and cerebellar hypoplasia. In addition to this, we found novel compound heterozygote variants including deletions and frameshift mutations in 14 additional unrelated families with 17 affected individuals sharing overlapping clinical symptoms. We observed that patient iPSC-derived neurons expressing GEMIN5 variants drastically reduced the expression of snRNP components (SMN, Gemin2, Gemin4 and Gemin6) as compared to controls suggesting a potential disruption in snRNP complex. Pathogenic mutations in GEMIN5 as well as shRNA mediated knockdown of endogenous GEMIN5 perturbed snRNP complex assembly.
Importantly, knocking down rigor mortis, fly homologue of human GEMIN5, showed a reduced lifespan and motor dysfunction similar to human patients. Furthermore, we identified transcriptomic changes that are caused by pathogenic mutations in GEMIN5 in patients. Here, we provide the first evidence that, patients carrying autosomal recessive mutations in GEMIN5 display neurological symptoms. Our data suggests that pathogenic mutations in GEMIN5 perturb the physiological functions by potential loss-of-function of GEMIN5 protein.

U. Pandey: None.

\section{C29 Skin \& Bones}

\section{C29.1}

Homozygous loss-of-function mutations in $C C D C 134$ are responsible for a severe form of osteogenesis imperfecta

J. Dubail ${ }^{*}$, P. Brunelle ${ }^{l}$, G. Baujat ${ }^{l}$, C. Huber ${ }^{l}$, M.

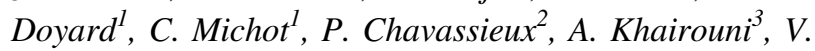
Topouchian $^{4}$, S. Monnot ${ }^{1}$, E. Koumakis ${ }^{1,5}$, V. CormierDaire $^{1}$

${ }^{1}$ Institut Imagine INSERM U1163, Paris, France, ${ }^{2}$ Université de Lyon, INSERM UMR 1033, Lyon, France, ${ }^{3}$ Pediatric Orthopedics, Casablanca, Morocco, ${ }^{4}$ Necker-Enfants Malades Hospital, Paris, France, ${ }^{5}$ Cochin Hospital, Rheumatology department, Paris, France

Osteogenesis Imperfecta (OI) is a primary bone fragility disorder with an estimated prevalence of 1/15 000 births. The majority of OI cases are inherited in an autosomaldominant manner, while five to ten percent have recessive or X-linked inheritance. Up to now, approximately $10 \%$ of OI cases remain without mutation demonstrated, supporting the involvement of other genes in the disease spectrum. By whole exome sequencing, we identified a homozygous variant (c.2T>C) in CCDC134 gene in three patients from two unrelated families, with bisphosphonate-resistant severe bone fragility, short stature, gracile long bones with pseudarthroses, but no dentinogenesis imperfecta. CCDC134 encodes a secreted protein widely expressed, implicated in the regulation of some mitogen-activated protein kinases (MAPK) signaling pathway. Western blot and immunofluorescence analyses confirmed the absence of CCDC134 protein in patient cells, compared to controls. Furthermore, we demonstrated that $C C D C 134$ mutations are associated with increased Erk1/2 phosphorylation and decreased COLIAI and OPN mRNA expression in patient osteoblasts compared to controls. These data support that $C C D C 134$ is a new gene involved in severe progressive deforming recessive osteogenesis imperfecta (type III). 
J. Dubail: None. P. Brunelle: None. G. Baujat: None. C. Huber: None. M. Doyard: None. C. Michot: None. P. Chavassieux: None. A. Khairouni: None. V. Topouchian: None. S. Monnot: None. E. Koumakis: None. V. CormierDaire: None.

\section{C29.2}

Hypomorphic variants in the replication complex member GINS3 result in a Meier Gorlin phenotype

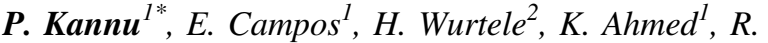

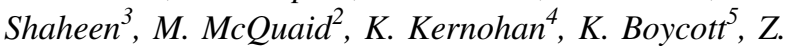 Alhassnan $^{3}$, F. Alkuraya ${ }^{3}$}

${ }^{1}$ The Hospital for Sick Children, Toronto, ON, Canada,

${ }^{2}$ Université de Montréal, Montreal, QC, Canada, ${ }^{3}$ King

Faisal Specialist Hospital, Riyadh, Saudi Arabia, ${ }^{4}$ Children's Hospital of Eastern Ontario, Toronto, ON, Canada, ${ }^{5}$ Children's Hospital of Eastern Ontario, Ottawa, ON, Canada

Introduction: Meier-Gorlin syndrome (MGORS) is a rare genetic disorder characterized by primordial dwarfism, microtia, and patellar aplasia//hypoplasia, which is caused by impaired DNA replication. Here we report the identification of four individuals from two unrelated families presenting a MGORS like phenotype, characterized by unreported recessive hypomorphic variants in the GINS3 gene, which encodes a subunit of the CMG helicase. The evolutionarily conserved CDC45/MCM2-7/GINS1-4 (CMG) protein complex is an essential helicase that unwinds DNA during DNA replication.

Results: We found that variants in GINS3 limit origin of replication firing, hence slowing cellular proliferation, providing a potential mechanism for the dwarfism phenotype. Furthermore, the variants destabilize the GINS subcomplex, thereby affecting the entire DNA replication fork altogether. Cells with the GINS3 variants accumulate in the $\mathrm{S}$ and $\mathrm{G} 2$-phases of the cell cycle. Infection of patient cells with a lentivirus expressing wt GINS3 rescues the origin of firing of replication forks and proliferation rates. We also show that the GINS3 variants alter protein-protein interactions within components of the replication machinery and are hypersensitive to DNA damage.

Conclusions: In summary, we have identified variants in GINS3 as a new cause of MGORS and established GINS3 as a critical component required for efficient DNA replication and genome stability.

P. Kannu: D. Speakers Bureau/Honoraria (speakers bureau, symposia, and expert witness); Modest; Alexion, Ipsen. F. Consultant/Advisory Board; Modest; Ipsen. E. Campos: None. H. Wurtele: None. K. Ahmed: None. R.
Shaheen: None. M. McQuaid: None. K. Kernohan: None. K. Boycott: None. Z. Alhassnan: None. F. Alkuraya: None.

\section{C29.3}

Homozygous EMILIN1 loss-of-function variants impair both elastin and collagen fiber formation and cause a novel entity with arterial tortuosity and osteopenia

A. Beyens ${ }^{1,2^{*}}$, C. Adamo ${ }^{3,4}$, E. Yilmaz Gulec ${ }^{5}$, A. Gezdirici ${ }^{5}$, P. Bonaldo ${ }^{6}$, H. Bornaun ${ }^{7}$, E. Brauchle ${ }^{8}$, J. Brinckmann ${ }^{9}$, W. P. Devine ${ }^{10}$, B. Gangaram ${ }^{11}$, T. Sasaki ${ }^{12}$, K. SchenkeLayland $^{8,13}$, S. Symoens ${ }^{1}$, A. Tam ${ }^{11}$, G. Sengle ${ }^{3,4,14}$, B. Callewaert $^{1}$

${ }^{1}$ Center for Medical Genetics Ghent and Department of Biomolecular Medicine, Ghent University Hospital, Ghent, Belgium, ${ }^{2}$ Department of Dermatology, Ghent University Hospital, Ghent, Belgium, ${ }^{3}$ Center for Biochemistry, Faculty of Medicine, University of Cologne, Cologne, Germany, ${ }^{4}$ Department of Pediatrics and Adolescent Medicine, Faculty of Medicine, University Hospital of Cologne, Cologne, Germany, ${ }^{5}$ Department of Medical Genetics, Kanuni Sultan Suleyman Training and Research Hospital, Health Sciences University, Istanbul, Turkey, ${ }^{6}$ Department of Molecular Medicine, University of Padova, Padova, Italy, ${ }^{7}$ Department of Pediatric Cardiology, Kanuni Sultan Suleyman Training and Research Hospital, Health Sciences University, Istanbul, Turkey, ${ }^{8}$ Research Centre for Women's Health, University of Tübingen, Tübingen, Germany, ${ }^{9}$ Institute of Virology and Cell Biology, University of Lübeck, Lübeck, Germany, ${ }^{10}$ Pathology Department, University of California San Francisco, San Francisco, CA, United States, " Department of Pediatrics, University of California San Francisco, San Francisco, CA, United States, ${ }^{12}$ Department of Biochemistry II, Faculty of Medicine, Oita University, Oita, Japan, ${ }^{13}$ NMI Tübingen, University of Tübingen, Tübingen, Germany, ${ }^{14}$ Center for Molecular Medicine Cologne (CMMC), University of Cologne, Cologne, Germany

Introduction: EMILIN1 belongs to the Elastin Microfibril Interface-Located Proteins (EMILINs), a family of structurally related extracellular glycoproteins. It is found in various tissues characterized by pronounced resilience and elastic recoil, such as skin and arteries, where it associates with elastic fibers and preferentially targets fibrillin microfibrils. EMILIN1 has been reported to bind and co-localize with fibulin-4 in the extracellular matrix. Fibulin-4 is a known carrier of lysyl oxidase (LOX), an enzyme involved in elastin and collagen crosslinking. Arterial tortuosity can be found in several connective tissue disorders, such as 
FBLN4-related cutis laxa and arterial tortuosity syndrome (ATS), caused by SLC2A10 mutations.

Results: Exome sequencing in two unrelated families with arterial tortuosity revealed homozygous loss-of-function mutations in EMILIN1. Patients exhibit widespread arterial tortuosity and fractures of ribs and long bones. Immunofluorescence of patient fibroblasts reveals a direct dependence of fibulin-4 fiber assembly on EMILIN1 deposition. Western blotting and mass spectrometry analysis show altered levels of LOX and collagen 1. Raman microspectroscopy analysis carried out in in Emilin $1^{-/}$mice shows structural changes in the collagen and elastic fiber networks. Biochemical analysis of human fibroblast cultures and Emilin ${ }^{-/}$mice skin demonstrates a disturbed collagen crosslinking pattern. Transmission electron microscopy of patient skin displays a frayed and moth-eaten aspect of the elastin with disorganization of microfibrillar structures. Collagen fibrils show increased and more variable diameters.

Conclusion: This study describes a novel syndrome associated with arterial tortuosity and suggests a mechanism through which altered elastic fiber homeostasis affects collagen fiber formation. (Grant Reference BOF01N04516)

A. Beyens: None. C. Adamo: None. E. Yilmaz Gulec: None. A. Gezdirici: None. P. Bonaldo: None. H. Bornaun: None. E. Brauchle: None. J. Brinckmann: None. W. P. Devine: None. B. Gangaram: None. T. Sasaki: None. K. Schenke-Layland: None. S. Symoens: None. A. Tam: None. G. Sengle: None. B. Callewaert: None.

\section{C29.4}

Transcriptional single-cell atlas of human limb malformation candidate genes

C. A. Prada-Medina ${ }^{1 *}$, G. Cova $^{l}$, J. Glaser ${ }^{1}$, C. Dietrich ${ }^{l}$, J. Henck ${ }^{1}$, S. Mundlos ${ }^{1,2}$, M. A. Mensah ${ }^{2}$, M. Spielmann ${ }^{1,2}$

${ }^{1}$ Max Planck Institute for Molecular Genetics, Berlin, Germany, ${ }^{2}$ Institute for Medical Genetics and Human Genetics Charité, Berlin, Germany

The extensive clinical and genetic heterogeneity of congenital limb malformation requires a comprehensive analysis of genome-wide genetic variation. Large exome and genome sequencing studies have led to the identification of hundreds of candidate genes. However, few variants have been functionally characterized. The sheer number of these candidate genes makes traditional functional analyses e.g., in animal models almost impossible. Therefore, for the vast majority of patients, the molecular mechanism is still unknown. In our study, we used high throughput single-cell transcriptomics to create a reference atlas of candidate genes during embryonic limb development. We characterized gene expression of over fifty thousand cells from four critical days of mouse early limb development (E9.5, E10.5, E11.5, and E12.5) by performing a single-cell RNA sequencing. We implemented an unsupervised approach to identify the general transcriptional patterns of diseasecausing genes during mouse embryonic limb development. We curated a gene-set of over 1400 genes that are associated with limb malformation in mice. $25 \%$ of these genes ranked among the topmost informative gene expression variances during early limb development. Over sixty of these genes present cell-type-specific gene expression patterns, mainly in the ectoderm and the apical ectodermal ridge. We show that mutations in genes expressed during early limb development are particularly prone to give rise to limb malformation. Our study provides an atlas of candidate genes that are co-expressed with known limb disease genes and represents a resource to study cell-types and molecular mechanisms involved in limb malformation.

C. A. Prada-Medina: None. G. Cova: None. J. Glaser: None. C. Dietrich: None. J. Henck: None. S. Mundlos: None. M. A. Mensah: None. M. Spielmann: None.

\section{C29.5}

Tendon-to-bone attachment cells are bi-fated $<$ and $>$ are regulated by shared enhancers <and $>$ KLF transcription factors

S. Kult ${ }^{1}$, T. Olender ${ }^{1 *}$, M. Osterwalder ${ }^{2}, S$. Krief $^{l}, R$. Blecher-Gonen ${ }^{1}$, S. Ben-Moshe ${ }^{l}$, L. Farack', H. KerenShaul $^{l}$, D. Leshkowitz ${ }^{1}$, T. M. Salame ${ }^{I}$, T. D. Capellini ${ }^{3}$, S. Itzkovitz ${ }^{1}$, I. Amit ${ }^{1}$, A. Visel ${ }^{2}$, E. Zelzer ${ }^{1}$

${ }^{1}$ The Weizmann Instiute of Science, Rehovot, Israel, ${ }^{2}$ Lawrence Berkeley National Laboratory, Berkeley, CA, United States, ${ }^{3}$ Harvard University, Cambridge, MA, United States

The mechanical challenge of attaching elastic tendons to stiff bones is solved by the formation of a unique transitional tissue, the enthesis. A comprehensive molecular signature of the developing enthesis cells has yet been uncovered. Here, we show that tendon-to-bone attachment cells are bi-fated, activating a mixture of chondrocyte and tenocyte transcriptomes, under regulation of shared regulatory elements and Krüppel-like factors transcription factors (KLFs). RNA and ATAC sequencing of embryonic attachment cells, chondrocytes and tenocytes revealed expression of both chondrogenic and tenogenic genes by attachment cells. Indeed, in situ hybridization and singlemolecule ISH showed that attachment cells co-express tenocyte and chondrocyte markers. ATAC-Seq analyses combined with ENCODE histone mark data, conservation, and other datasets revealed that attachment cells are regulated by putative enhancers that are shared with either 
tenocytes or chondrocytes. Transgenic mouse enhancer reporter assay verified the shared activity of three enhancers, supporting the notion that the mixed transcriptome of attachment cells is regulated by sharing enhancers with tenocytes or chondrocytes. Combining over-represented transcription factor analysis with transcriptome results suggested a regulatory role for KLFs in attachment cells. Indeed, blocking expression of Klf2 and Klf4 in the developing limb mesenchyme impaired attachment cell differentiation, establishing that KLFs are key regulators of the fate of these cells. Overall, we provide the first molecular overview of the developing transitional tissue, connecting tendon to bone, showing that activation of a combination of cartilage and tendon transcriptomes by the bi-fated attachment cells leads to the formation of a new, intermediate cell fate.

S. Kult: None. T. Olender: None. M. Osterwalder: None. S. Krief: None. R. Blecher-Gonen: None. S. Ben-Moshe: None. L. Farack: None. H. Keren-Shaul: None. D. Leshkowitz: None. T. M. Salame: None. T. D. Capellini: None. S. Itzkovitz: None. I. Amit: None. A. Visel: None. E. Zelzer: None.

\section{C29.6}

Identifying targets for osteoporosis via whole exome sequencing and CRISPR-Cas9

\author{
S. Zhou ${ }^{l *}$, L. Laurent ${ }^{l}$, J. P. Kemp ${ }^{2}$, V. Forgetta ${ }^{I}, J$. \\ Morris $^{3}$, A. Pramatarova ${ }^{4}$, P. I. Croucher ${ }^{5}$, J. Bassett ${ }^{6}, D$. \\ Goltzman $^{7}$, D. M. Evans ${ }^{2}$, J. Richards ${ }^{1}$
}

${ }^{1}$ McGill University, Jewish General Hospital, Montreal, QC, Canada, ${ }^{2}$ University of Queensland Diamantina Institute, Translational Research Institute, Brisbane, Australia, ${ }^{3}$ New York Genome Center, New York, NY, United States, ${ }^{4}$ McGill University and Genome Quebec Innovation Centre, Montreal, QC, Canada, ${ }^{5}$ Garvan Institute of Medical Research, Sydney, Australia, ${ }^{6}$ Molecular Endocrinology Laboratory, Department of Medicine, Imperial College London, London, United Kingdom, ${ }^{7}$ Research Institute of the McGill University Health Centre, Montreal, QC, Canada

New drug targets are needed for osteoporosis. It is not known whether whole exome sequencing (WES) or GWAS followed by target gene mapping can better identify causal genes for this disease. To compare these approaches, we first identified a set of 54 positive control genes whose perturbation causes a Mendelian form of osteoporosis, or which act as drug targets for approved therapies. We undertook sequence kernel association tests (SKATO) to associate 18,350 autosomal protein-coding genes with estimated bone mineral density (eBMD), using WES data of 42,404 white British participants from UK Biobank and performed a GWAS in the same sample. We found that WES identified genes were more strongly enriched for positive control genes (odds ratio [OR] $=119$, $\mathrm{P}=5.2 \times 10^{-6}$ ), and this enrichment was 5-fold higher compared to enrichment of GWAS-identified genes (OR = $22.5, \mathrm{P}=5 \times 10^{-5}$ ). From WES data, we identified 38 genes with exonic variants with functional impact that collectively associated with eBMD, one of the most prominent genes was $C A D M 1$ (SKATO, $\mathrm{P}=1.8 \times 10^{-9}$ ). We used CRISPRCas9 to knockout (KO) CADM1 in SaOS-2 osteoblast cell lines, suppressing protein abundance at the cell surface, which resulted in increased expression of early bone markers: RUNX2, COL1A1/2 and ALPL. We previously demonstrated that $C a d m 1$ KO mice had reduced bone mineral content ( -3.2 standard deviations [SD]) and strength (-2.0 SD) compared to wildtype. These findings demonstrate that WES is a powerful approach to identify genes influencing bone density, such as $C A D M 1$, which could serve as targets for osteoporosis therapies.

S. Zhou: None. L. Laurent: None. J. P. Kemp: None. V. Forgetta: None. J. Morris: None. A. Pramatarova: None. P. I. Croucher: None. J. Bassett: None. D. Goltzman: None. D. M. Evans: None. J. Richards: None.

\section{C30 Polygenic Risk Scores: From Tool to Practice}

\section{C30.1}

Using reference-fixed principal components to improve polygenic risk score prediction

\section{o. Naret ${ }^{*}$, J. Fellay}

École polytechnique fédérale de Lausanne, Lausanne, Switzerland

Genome-wide association studies (GWAS) have identified multiple genetic variants associated with traits or diseases. They confirmed that most common diseases have a strong genetic component coming from many genetic variants, each having a small effect size on disease risk. Using the summary statistics from highly powered GWAS, it is now possible to build polygenic risk scores (PRS) that can help predict the individual risk of common diseases. We here propose to improve PRS prediction by leveraging population stratification due to demographic history, which is a typical confounding factor removed during GWAS. We provide a tool that 1) map a discovery cohort to the principal component (PC) space of the 1000 Genomes Project cohort and train a model to associate the phenotype distribution to this PC space; 2) map a target patient to the same PC space and use the previously trained model to compute its PC score. We compared two disease risk 
models, one using PRS only and one using PRS and PC score jointly. Applying this method to UK Biobank data, we demonstrate improvement for all tested phenotypes: $<10 \%$ for menarche age, blood pressure, BMI, baldness, and height), $14 \%$ for menopause age, $50 \%$ for diabetes, $59 \%$ for bone mineral density and $455 \%$ for skin color. This approach has the potential to allow ethnically diverse populations to benefit from genomic medicine.(Grant: SNF:175603)

O. Naret: None. J. Fellay: None.

\section{C30.2}

The Polygenic Score (PGS) Catalog: an open database to enable reproducibility and systematic evaluation

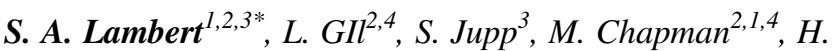
Parkinson $^{3,2}$, J. Danesh ${ }^{1,2,5,4}$, J. A. L. MacArthur ${ }^{3}$, M. Inouye $e^{1,2,5,6,7}$

${ }^{1}$ University of Cambridge, Cambridge, United Kingdom,

${ }^{2}$ Health Data Research UK-Cambridge, Wellcome Genome Campus, Hinxton, United Kingdom, ${ }^{3}$ European Molecular Biology Laboratory, European Bioinformatics Institute, Wellcome Genome Campus, Hinxton, United Kingdom, ${ }^{4}$ Wellcome Sanger Institute, Wellcome Genome Campus, Hinxton, United Kingdom, ${ }^{5}$ National Institute for Health Research Cambridge Biomedical Research Centre at the Cambridge University Hospitals NHS Foundation Trust, Cambridge, United Kingdom, ${ }^{6}$ Baker Heart and Diabetes Institute, Melbourne, Australia, ${ }^{7}$ The Alan Turing Institute, London, United Kingdom

Introduction: Polygenic [risk] scores (PGS), which aggregate hundreds-to-millions of genomic variant's effects into a single score, can enhance prediction and understanding of common diseases and traits. Rapidly-evolving research into PGS and their potential clinical utility has, however, been limited by inadequate and/or incomplete reporting, heterogeneity in evaluation techniques, and variable performance in populations of non-European ancestry.

Objective: An accurate understanding of each PGS and its predictive value is necessary for its application. Collaborating with the NHGRI-EBI GWAS Catalog, we have developed the PGS Catalog to provide an open resource of consistently annotated PGS, to increase their reproducibility and availability.

Methods: We developed a framework to summarise and represent PGS. For each PGS we extract metadata describing the score (rsID, effect allele \& weight), its development and application details, links to GWAS Catalog summary statistics employed, characteristics of training/validation cohorts (phenotyping, sample size, ancestry), and reported performance metrics.

Results: The current PGS Catalog contains over 100 consistently annotated PGS for 43 different traits, distributed in an open database (www.PGSCatalog.org), initially focusing on purpose-built PGS developed after 2010 for cardiometabolic diseases and cancers. The Catalog is regularly updated to broaden its coverage, and we invite researchers to submit their PGS to maximize their utility. We aim to provide a comprehensive resource of available PGS along with a complete picture of their predictive accuracy - to this end we outline our upcoming efforts to systematically evaluate the cataloged PGS in diverse external cohorts, in order to distribute directly comparable measures of PGS performance.

S. A. Lambert: None. L. GIl: None. S. Jupp: None. M. Chapman: None. H. Parkinson: None. J. Danesh: F. Consultant/Advisory Board; Modest; Novartis, Sanofi, Astra Zeneca. J. A. L. MacArthur: None. M. Inouye: None.

\section{C30.3}

Polygenic risk, breast cancer susceptibility genes, and breast cancer over the life course

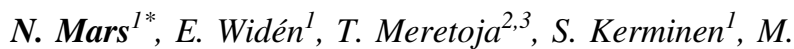
Pirinen $^{1,4,5}$, P. Palta ${ }^{1,6}$, A. Palotie ${ }^{1,7,8}$, J. Kaprio ${ }^{1,5}$, H. Joensuu $^{9,3}$, M. Daly ${ }^{1,8}$, S. Ripatti ${ }^{1,5,8}$

${ }^{I}$ Institute for Molecular Medicine Finland (FIMM), HiLIFE, University of Helsinki, Helsinki, Finland, ${ }^{2}$ Breast Surgery Unit, Comprehensive Cancer Center, Helsinki University Hospital, Helsinki, Finland, ${ }^{3}$ University of Helsinki, Helsinki, Finland, ${ }^{4}$ Helsinki Institute for Information Technology HIIT and Department of Mathematics and Statistics, University of Helsinki, Helsinki, Finland, ${ }^{5}$ Department of Public Health, University of Helsinki, Helsinki, Finland, ${ }^{6}$ Estonian Genome Center, Institute of Genomics, University of Tartu, Tartu, Estonia, ${ }^{7}$ Psychiatric \& Neurodevelopmental Genetics Unit, Department of Psychiatry, Analytic and Translational Genetics Unit, Department of Medicine, and the Department of Neurology, Massachusetts General Hospital, Boston, MA, United States, ${ }^{8}$ Broad Institute of the Massachusetts Institute of Technology and Harvard University, Cambridge, MA, United States, ${ }^{9}$ Comprehensive Cancer Center, Helsinki University Hospital, Helsinki, Finland

Breast cancer is the most common cancer in women, with both monogenic and polygenic germline inheritance. Among 99,969 women in FinnGen, we set out to evaluate how breast cancer polygenic risk score (PRS) and highimpact frameshift variants jointly impact the risk of breast cancer. Secondly, we evaluated whether PRS impacts breast 
cancer mortality in the population, and the risk of recurrence and mortality among breast cancer patients.

The lifetime risk of breast cancer (by age 80) for carriers of two Finnish-enriched frameshift variants, c.1592delT in PALB2 (242-fold enrichment in Finland, 270 carriers) and c.1100delC in CHEK2 (3.7-fold enrichment, 1,543 carriers) was $57 \%$ and $32 \%$, respectively. The PRS modifies the breast cancer risk conferred by the two variants. Women with PALB2 and average PRS (20-80th percentile) had a lifetime risk of $56 \%$, which increased to $85 \%$ with high PRS ( $>90$ th percentile), and decreased to $27 \%$ with low PRS $(<20$ th percentile). For $C H E K 2$, these lifetime risks were $27 \%, 59 \%$, and $18 \%$.

We move beyond the simple binary definition of breast cancer: the PRS was associated with a more severe disease, both in the population (HR 2.25, 95\%CI 1.72-2.95 for breast cancer-related mortality with a PRS $>90^{\text {th }}$ percentile), and in breast cancer cases (HR 1.21, 95\% CI 1.09-1.34 for risk of breast cancer recurrence; similar results adjusting for surgery type, age, and stage at diagnosis).

We demonstrate opportunities to improve genetic risk assessment in breast cancer, both using PRS alone and for facilitating better management of women carrying mutations in breast cancer susceptibility genes.

N. Mars: None. E. Widén: None. T. Meretoja: None. S. Kerminen: None. M. Pirinen: None. P. Palta: None. A. Palotie: None. J. Kaprio: None. H. Joensuu: None. M. Daly: None. S. Ripatti: None.

\section{C30.4}

\section{Genetic assessment of age-associated fracture risk}

C. Medina-Gomez ${ }^{l^{*}}, K$. Trajanoska ${ }^{l}$, C. Fan ${ }^{2}$, O. A. Andreassen $^{3}$, M. Zillikens ${ }^{1}$, A. M. Dale ${ }^{2}$, A. G. Uitterlinden $^{1}$, T. M. Seibert ${ }^{2}$, F. Rivadeneira ${ }^{1}$

${ }^{1}$ ErasmusMC, Rotterdam, Netherlands, ${ }^{2}$ University of California, San Diego, CA, United States, ${ }^{3}$ University of Oslo, Oslo, Norway

Aim: Identifying individuals at risk of early-onset osteoporosis is of outmost importance. The largest GWAS to date, identified 1,103 genetic variants associated with ultrasound-derived Bone Mineral density (eBMD). We investigated whether a genetic risk score (GRS) from these variants would associate with fracture incidence and predict time to fracture.

Methods: We included in this study 11,351 participants of the Rotterdam Study with up to 20 years of follow-up. Incident non-vertebral fractures were reported in 2,153 (19\%) individuals. GRS was constructed as a weighted sum of the number of putative eBMD-decreaser alleles at 1,031 genetic variants. Cox regression and Weibull survival analysis were implemented adjusting for sex.

Results: An increment of one standard deviation (SD) in the GRS represented an increase of $1.20\left(\mathrm{pv}<2 \times 10^{-16}\right)$ in the hazard ratio of fracture. When analyzed in quintiles, individuals in the highest quintile had $1.52 \mathrm{HR}$ of fracture (95\% CI 1.09-2.11) as compared to these individuals in the middle quintile, despite their similitude in anthropometric characteristics (Table 1). In addition, fracture occurred significantly earlier in the former group (event time ratio $=$ 0.95, 95\%CI 0.91-0.99).

Conclusion: Our results suggest that GRS can be used for personalized genetic risk estimates that can predict for age at fracture. We show how a suboptimal epidemiological predictive tool, as ultrasound measurements for fracture prediction, is leveraged within the genetic framework.

Table 1

\begin{tabular}{lllllll}
\hline Quintiles GRS & $1 \mathrm{~N}=54$ & $2 \mathrm{~N}=1758$ & $3 \mathrm{~N}=6497$ & $4 \mathrm{~N}=2892$ & $5 \mathrm{~N}=150$ & $\mathrm{P}$ \\
\hline Fracture $\%$ & $9.2 \%$ & $14.1 \%$ & $18.7 \%$ & $22.4 \%$ & $24.7 \%$ & $1.8 \mathrm{E}-11$ \\
HR & 0.6 & 0.7 & Ref & 1.2 & 1.5 & $<2 \mathrm{E}-16$ \\
ETR & 1.07 & 1.04 & Ref & 0.97 & 0.95 & $<2 \mathrm{E}-16$ \\
FN-BMD $(\mathrm{g} / \mathrm{cm} 2)$ & 1.00 & 0.94 & 0.91 & 0.87 & 0.83 & $<2 \mathrm{E}-16$ \\
Female\% & $64.0 \%$ & $58.0 \%$ & $57.0 \%$ & $59.0 \%$ & $58.0 \%$ & 0.47 \\
Age (yrs) & 63.0 & 65.5 & 65.2 & 65.4 & 65.2 & 0.86 \\
Weight $(\mathrm{kg})$ & 76.5 & 75.8 & 76.1 & 76.5 & 77.0 & 0.23 \\
Height $(\mathrm{m})$ & 1.67 & 1.68 & 1.68 & 1.68 & 1.69 & 0.51 \\
\hline
\end{tabular}

C. Medina-Gomez: None. K. Trajanoska: None. C. Fan: None. O. A. Andreassen: None. M. Zillikens: None. A. M. Dale: None. A. G. Uitterlinden: None. T. M. Seibert: None. F. Rivadeneira: None.

\section{C30.5}

Integrative analysis of the plasma proteome and polygenic risk of cardiometabolic disease

S. C. Ritchie ${ }^{\text {* }}$, Y. Liu ${ }^{2}$, S. A. Lambert ${ }^{1}$, B. G. Drew ${ }^{2}$, A. C. Calkin $^{2}$, A. S. Butterworth ${ }^{1}$, J. Danesh ${ }^{l}$, M. Inouye ${ }^{1,2}$

${ }^{1}$ University of Cambridge, Cambridge, United Kingdom, ${ }^{2}$ Baker Heart and Diabetes Institute, Melbourne, Australia

Introduction: Polygenic risk scores (PRSs) capture the genetic architecture of common diseases by aggregating genome-wide genetic variation into a single score that reflects an individual's disease risk. These present new opportunities to identify molecular pathways involved in disease pathogenesis.

Materials and Methods: We performed association analysis between PRSs and 3,442 plasma proteins using linear regression models in 3,175 healthy individuals to identify proteins whose levels associated with PRSs for 
atrial fibrillation, coronary artery disease, chronic kidney disease, stroke, or type 2 diabetes. Mendelian Randomisation analysis was used to test whether levels of PRSassociated proteins were causal of disease risk. Tissuespecific gene expression levels of PRS-associated proteins were tested for correlations with cardiometabolic phenotypes in mouse models. We investigated potential drug repurposing opportunities by overlaying PRS to protein associations with drug target information.

Results: We identified 48 proteins whose levels associated with PRSs for coronary artery disease, chronic kidney disease, or type 2 diabetes, including well-known disease-associated proteins along with novel associations. Mendelian Randomisation supported a causal effect for changes in levels of three PRS-associated proteins on disease. Mouse models revealed strong correlations with relevant cardiometabolic traits for tissue-specific expression of seven PRS associated proteins with no previous known link to cardiometabolic disease. Finally, we identified three promising potential drug repurposing opportunities where the effect of on-market drugs on protein levels were consistent with PRS to protein associations.

Conclusions: Overall, our study provides a framework for polygenic association studies, demonstrating the power of PRSs to identify and prioritise novel disease biology.

S. C. Ritchie: None. Y. Liu: None. S. A. Lambert: None. B. G. Drew: None. A. C. Calkin: None. A. S. Butterworth: None. J. Danesh: None. M. Inouye: None.

\section{C30.6}

Genotyping On ALL patients (GOALL); clinical implementation of high-throughput genotyping arrays

J. van Rooij , A. Verkerk, B. Sedaghati-khayat, L. Broer, J. de Vries, G. van Dijk, J. van Meurs, A. Uitterlinden

\section{Erasmus Medical Centre, Rotterdam, Netherlands}

Introduction: Genetic testing is increasingly used in standard clinical practice. Dense genotyping arrays cover a large portion of known clinically relevant genetic variation and provide a cost-effective, high-throughput and highly standardized alternative. In the GOALL project, we are determining the clinical utility and applications of genotyping arrays.

Methods: We investigate the clinical utility of the GSAMD_v2 and PMDA arrays by: 1) technical validation of common and rare variants 2) offering polygenic risk scores (PRS) as primary utility in clinical practice, 3) prospectively counseling and testing eligibility of feeding back secondary findings (pharmacogenomics, ACMG mutations) using array based genotyping. We are piloting these applications in collaboration with multiple departments at the Erasmus MC.

Results: Our preliminary results suggest that $>90 \%$ of the mutations routinely determined in our hospital are covered by the array design. We have selected 240 known breast cancer mutation carriers for array genotyping to check concordance. Comparison of array vs. WES of 197 population samples shows an overall non-reference concordance of $98 \%$, and $95 \%$ for singletons. Population pilot results demonstrate significant primary case stratification by PRS for breast cancer, coronary artery disease, myocardial infarction, osteoarthritis and age-related macular degeneration.

Discussion: Our preliminary results suggest that a portion of hospital-wide clinical genetic testing can cost-effectively be replaced by array-based genotyping. In addition to reporting highly concordant results, array-based genotyping allows additional reporting of several PRS, HLA- and ancestry-typing and pharmacogenomics. This data can easily be stored and re-analyzed, or unblinded when a patient requires further (secondary) genetic testing.

J. van Rooij: None. A. Verkerk: None. B. Sedaghatikhayat: None. L. Broer: None. J. de Vries: None. G. van Dijk: None. J. van Meurs: None. A. Uitterlinden: None.

\section{C31 Shaping the Genomic Medicine Era}

\section{C31.1}

"It's probably nothing, but..." Couples' experiences of pregnancy following a uncertain prenatal genetic result

\author{
S. Lou $^{1,2^{*}}$, K. Lomborg ${ }^{3}$, C. Lewis ${ }^{4}$, S. Riedijk ${ }^{5}$, O. \\ Petersen $^{6}$, I. Vogel ${ }^{1,7}$
}

${ }^{1}$ Center for Prenatal Diagnostics, Aarhus University Hospital, Aarhus, Denmark, ${ }^{2}$ Defactum - Public Health \& Health Services Research, Aarhus, Denmark, ${ }^{3}$ Steno Diabetes Center Copenhagen, Copenhagen, Denmark, ${ }^{4}$ London North Genomic Laboratory Hub, Great Ormond Street Hospital, London, United Kingdom, ${ }^{5}$ Erasmus Medical Center, Rotterdam, Netherlands, ${ }^{6}$ Fetal Medicine Unit, Department of Obstetrics \& Gynecology, Rigshospitalet Copenhagen University Hospital, Copenhagen, Denmark, ${ }^{7}$ Department of Clinicial Genetics, Aarhus University Hospital, Aarhus, Denmark

Introduction: The introduction of Chromosomal MicroArray in prenatal testing includes the potential identification of uncertain copy number variants $(\mathrm{CNV})$ where significance and implications for the unborn child can be difficult or impossible to predict. The aim of this study was to explore how prospective parents manage uncertainty and experience pregnancy in light of an uncertain $\mathrm{CNV}$ result. 
Material and methods: Qualitative interviews with 16 women and 10 partners who had received a prenatally diagnosed, uncertain CNV. Participants were interviewed 114 weeks after birth. Data were analyzed using thematic analysis.

Results: Following the CNV diagnosis, some couples focused on the severe syndromes ruled out by the result, while others were more concerned with the new potential risks, e.g. learning disabilities. During pregnancy, the couples used various strategies to reduce worry such as limiting information seeking, reducing talk of the CNV and deferring thoughts of potential consequences. Furthermore, additional ultrasound was appreciated by all. Inherited CNVs caused relief, but also feelings of responsibility for the child's potential challenges. After birth, worry decreased considerably. However, all couples paid extra attention to the child's development, while also being alert to the risk wrongfully interpreting the child's development in terms of the CNV. Eleven couples appreciated knowing about the child's CNV. Five couples would rather not have known.

Conclusions: Health professionals should be mindful of terminology, remember to point out what has been ruled out by the CNV result, and discuss potential coping strategies with the couple. Funding: Novo Nordisk Foundation (Grant NNF16OC0018722)

S. Lou: B. Research Grant (principal investigator, collaborator or consultant and pending grants as well as grants already received); Modest; Novo Nordisk Foundation. K. Lomborg: None. C. Lewis: None. S. Riedijk: None. O. Petersen: None. I. Vogel: B. Research Grant (principal investigator, collaborator or consultant and pending grants as well as grants already received); Modest; Novo Nordisk Foundation.

\section{C31.2}

Additional Genomic Findings: towards a feasible, effective model of care

E. Lynch ${ }^{1,2}$, M. Martyn ${ }^{1,3,4^{*}}$, L. Lee $e^{1,3,4}$, A. Kanga-Parabia ${ }^{2}$, R. Weerasuriya ${ }^{1,3,4}$, R. Tytherleigh $h^{1,3}$, C. L. Gaff $f^{1,3,4}$

${ }^{1}$ Melbourne Genomics Health Alliance, Parkville, Australia, ${ }^{2}$ Victorian Clinical Genetics Services, Parkville, Australia, ${ }^{3}$ Murdoch Children's Research Institute, Parkville, Australia, ${ }^{4}$ Department of Paediatrics, University of Melbourne, Parkville, Australia

Genomic sequencing for diagnosis makes possible deliberate analysis for variants predictive of future health risk ('additional findings'). Challenges to provision of AF in public health systems exist but evidence to guide development of a feasible, effective model of care is lacking. When available, AF are offered concurrently with diagnostic testing. To examine decisions about AF and investigate other models of care, we offered AF to 200 adults at a separate genetic counselling appointment after completion of diagnostic testing (i.e. after results)Patients completed surveys after AF genetic counselling, after AF result return and 6 months following last contact with the service. Consultations were recorded and analysed. Participating genetic counsellors were interviewed. Eighty three $(83 / 200$, $42 \%)$ patients attended pre-test counselling with $81(98 \%)$ accepting AF. Patients felt supported in decision making: $79 / 83(95 \%)$ agreed the level of information they received was 'about right' and 73/78 (94\%) reported counselling was helpful. Participants and counsellors identified valuable elements of counselling, including establishing a relationship. Genetic counsellors also reported addressing concerns and misconceptions yet, despite relatively intensive counselling, 34\% (28/83) of participants mistakenly believed the test was for all known genetic conditions or would provide information about their primary condition. All respondents to the post-result survey correctly recalled their AF result (49/49) but confusion about its implications was evident. Research is needed to identify communication strategies that effectively address common AF misconceptions and are achievable in public health systems in order to realise the ideal of a feasible, effective service for AF.

E. Lynch: None. M. Martyn: None. L. Lee: None. A. Kanga-Parabia: None. R. Weerasuriya: None. R. Tytherleigh: None. C. L. Gaff: None.

\section{C31.3}

Analysis of laboratory exome sequencing reporting practices using a 'virtual patient'

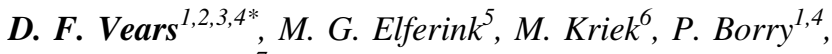 K. L. van Gassen 7

${ }^{1}$ Centre for Biomedical Ethics and Law, Department of Public Health and Primary Care,KU Leuven, Leuven, Belgium, ${ }^{2}$ University of Melbourne, Carlton, Australia, ${ }^{3}$ Murdoch Children's Research Institute, Parkville, Australia, ${ }^{4}$ Leuven Institute for Human Genomics and Society, Leuven, Belgium, ${ }^{5}$ University of Utrecht, Utrecht, Netherlands, ${ }^{6}$ Leiden University Medical Center, Leiden, Netherlands, ${ }^{7}$ University Medical Center Utrecht, Utrecht, Netherlands

Studies have suggested that laboratories performing exome sequencing return different types of results to clinicians. While some report variants of uncertain significance, unsolicited findings, and/or secondary findings, others do not. To investigate the degree of variation in reporting, we created a 'virtual patient-parent trio' by in silico merging 
eight disease-causing variants from patients into "normal" background exomes. We sent the sequence data and details of the patient's phenotype (developmental delay, dysmorphic features, cardiac hypertrophy), to laboratories worldwide and asked them to issue a diagnostic report. Participating laboratories completed a questionnaire to explain their rationale for (not) reporting each of the variants integrated. Of the 39 participating laboratories, 30 reported the $\mathrm{X}$-linked dominant inherited variant in the HDAC8 gene, which was the partial cause of the patient's phenotype, and 26 reported the de novo BICD2 variant, which explained another component of the phenotype. Laboratories who did not report these variants explained they used either a filter or a targeted gene panel that excluded the variant, or they did not consider the variant to be responsible for the phenotype. There was also variation in reporting associated with the cardiac phenotype; 30 reported MYBPC3 and only 16 reported PLN, often due to the filtering strategies used. Reporting of unsolicited/secondary findings also varied widely. We identified considerable variability in reporting of disease-causing variants, primarily due to different analysis strategies. This significantly impacts whether causative variants are identified, with important implications for patients. Analysis strategy best-practice guidelines may help reduce variability and improve patient care.

D. F. Vears: None. M. G. Elferink: None. M. Kriek: None. P. Borry: None. K. L. van Gassen: None.

\section{C31.4}

Knowledge, attitudes and preparedness for polygenic risk testing in clinical practice: a cross-sectional survey of health professionals

A. K. Smit ${ }^{*}$, A. E. Cust ${ }^{1}$, K. Dunlop ${ }^{1}$, M. Young ${ }^{2}, B$. Meiser $^{3}$, J. Tiller ${ }^{4}$, T. Yanes ${ }^{5,3}$

${ }^{1}$ The University of Sydney, Sydney, Australia, ${ }^{2}$ Garvan Institute of Medical Research, Sydney, Australia, ${ }^{3}$ University of New South Wales, Sydney, Australia, ${ }^{4}$ Monash University, Melbourne, Australia, ${ }^{5}$ University of Queensland, Woolloongabba, Australia

Introduction: Strategies to prepare and support health professionals for the potentialimplementation of polygenic risk scores (PRS) into clinical practice are required.

Materials and Methods: An anonymous questionnaire was distributed online byprofessional organisations in Australia, New Zealand, Canada and the US. Eligibleparticipants were health professionals who provide cancer risk assessments. Knowledge,perceived self-efficacy, concerns, benefits and expectations for the future clinical applicationof PRS were described. Content analysis was undertaken of free-text responses.

Results: A total of 128 health professionals took part in the questionnaire (geneticcounsellors $\mathrm{n}=86 \%$; oncologists $\mathrm{n}=5 \%$; clinical geneticists $\mathrm{n}=4 \%$; other $\mathrm{n}=5 \%$ ). Many haddiscussed PRS with a patient $(61 \%)$, but only $20 \%$ reported having ordered a test. Over half(69\%) reported that the use of PRS in future clinical practice was certain or likely. Many (49\%) reported that general practitioners would have a primary role in the provision of PRS, supported by genetics health professionals. Overall, confidence in explaining PRS to patientswas low, and participants reported concern about insufficient health professional resources,uncertainty about individual risk and barriers to obtaining life insurance. Perceived benefitsof PRS included greater incentive for patients to engage in preventive behaviours and accessto increased or tailored surveillance and screening.

Conclusions: Our findings demonstrate that health professionals are already providingpolygenic risk information, although fewer are ordering testing, and there is a need foreducational and point-of-contact resources to support this process. These findings will informthe development of implementation strategies for incorporating PRS into clinical practice.

A. K. Smit: None. A. E. Cust: None. K. Dunlop: None. M. Young: None. B. Meiser: None. J. Tiller: None. T. Yanes: None.

\section{C31.5}

Impact of the discussion of uncertainty on former counselees' outcomes. An experimental vignettes study

N. Medendorp ${ }^{1 *}$, M. Hillen ${ }^{l}, C$. Aalfs $^{2}$, A. Stiggelbout $^{3}$, E. Smets ${ }^{l}$

${ }^{1}$ Amsterdam UMC, Amsterdam, Netherlands, ${ }^{2}$ University Medical Center Utrecht, Utrecht, Netherlands, ${ }^{3}$ Leiden University Medical Center, Leiden, Netherlands

Introduction: Uncertainty increasingly needs to be discussed during cancer genetic counseling due to technological developments. How discussions about uncertainty affect counselees may depend on communication style. Ideally, counselees are enabled to deal with uncertainty and make well-informed decisions. We tested the impact of different ways of conveying uncertainty and addressing counselees' uncertainty on counselees' outcomes, and explored moderating effects of counselees' characteristics.

Materials and methods: Former counselees viewed one (out of six) randomly assigned video depicting a consultation involving the discussion of a multigene panel test. In a $2 \times 3$ design geneticists' communication of uncertainty 
(comprehensive vs. gist) and responses to counselees' uncertainty expressions (providing information vs. providing space vs. balancing uncertainty) were systematically manipulated. Before and after watching, participants completed questionnaires. Primary outcome was feelings of uncertainty.

Results: Preliminary analyses were performed on $91 \%$ $(n=178)$ of the required sample. Communication of uncertainty was successfully manipulated: the comprehensive version was perceived as more detailed ( $p=$. 099). However, differences in how responses to counselees' uncertainty expressions were not perceived $(p=.303)$. No effects on feelings of uncertainty were found for geneticists' communication about uncertainty $(p=.323)$, or response style $(p=630)$. Statistical analysis on the complete dataset, including effects on secondary outcomes and moderating effects of counselees' characteristics will be performed and discussed.

Conclusions: Preliminary analysis showed that different manners of communicating uncertainty and addressing counselees' uncertainty expressions did not lead to differences in feelings of uncertainty. Findings will inform a training intervention to support geneticists in discussing uncertainty.

Funding: Dutch Cancer Society (KWF Kankerbestrijding; UvA 2015-7607)

N. Medendorp: None. M. Hillen: None. C. Aalfs: None. A. Stiggelbout: None. E. Smets: None.

\section{C31.6}

Primary Care Genomics: A roadmap for curriculum change and educational resource development

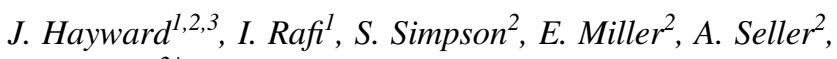 \\ M. Bishop ${ }^{2 *}$
}

${ }^{1}$ Royal College of General Practitioners, London, United Kingdom, ${ }^{2}$ Genomics Education Programme, Birmingham,
United Kingdom, ${ }^{3}$ Yorkshire and Humber Genomics Medicine Centre, Leeds, United Kingdom

Health Education England's Genomics Education Programme (GEP) has developed a roadmap for defining General Practitioners' (GPs) learning needs and identifying priorities for resource development, suitable for adoption by other specialties. With the implementation of England's National Health Service's (NHS) Genomic Medicine Service, genomic information will be used within routine clinical care by an increasing number of specialties. The GEP exists to ensure NHS staff are prepared for the integration of genomics into clinical practice. With the Royal College of General Practitioners (RCGP), the GEP devised a three-stage process - plan; develop; deliver - to identify GPs' learning requirements and appropriate interventions. Using the Delphi method, an expert working group $(n=30)$, comprising GPs, genetic specialists, patients and educationalists, identified 22 themes around the knowledge, skills and behaviours GPs require to manage clinical activities involving genomics (stage 1). Alongside this, a desktop exercise (stage 2) mapped genetic/genomic education resources relevant to GPs through targeted searches of organisational websites, supplemented by web searches. From these, resources from 21 organisations were identified. Next, a gap analysis (stage 3) mapped resources from stage 2 against the knowledge, skills and behaviours from stage 1 , establishing a list of resources requiring development. These resources, with those identified in stage 2, formed the foundation of an education toolkit, hosted on the RCGP website. Findings from stage 1 also informed the revised RCGP training curriculum for GPs. The toolkit has become a go-to destination for GPs learning about genomics, and its development roadmap can be adopted by other specialties.

J. Hayward: None. I. Rafi: None. S. Simpson: None. E. Miller: None. A. Seller: None. M. Bishop: None. 